TECHNOPOLIS

\title{
Evaluierung der Sonderaktion Holzforschung
}

im Auftrag des

Forschungsförderungsfonds

für die gewerbliche Wirtschaft

(FFF)

November 2002

Wilfried Puwein

Österreichisches Institut für Wirtschaftsforschung (WIFO)

Arsenal, Objekt 20

Postfach 91

1030 Wien

Tel. 017982601 - 241

wilfried.puwein@wifo.ac.at www.wifo.ac.at
Jörg Mahlich

Fritz Ohler Claudia Gamsjäger

Technopolis Forschungs- und Beratungsgesellschaft mbH Prinz Eugen Straße 80/12

1040 Wien

Tel. 015039592 - 16

joerg.mahlich@technopolis -group.com www.technopolis -group.com 


\section{Inhaltsverzeichnis}

1 Einleitung 1

2 Methode 3

3 Eckdaten des Programms $\quad 4$

3.1 Zahl der Förderwerber, Förderquote 4

3.2 Verteilung nach Branchen 5

3.3 Verteilung nach Bundesländern $\quad 6$

$\begin{array}{lll}3.4 & \text { Treffsicherheit der Förderkriterien und Projektbewertung } & \mathbf{7}\end{array}$

4 Charakterisierung der Förderwerber und der Kontrollgruppe 11

$\begin{array}{lll}4.1 & \text { Unternehmensgröße } & 11\end{array}$

$\begin{array}{lll}4.2 & \text { Exportaktivitäten } & 13\end{array}$

$\begin{array}{lll}4.3 & \text { Eigentumsverhältnisse } & 14\end{array}$

$\begin{array}{lll}4.4 & \text { F\&E-Verhalten } & 15\end{array}$

$\begin{array}{lll}4.5 & \text { Innovationsbarrieren } & 17\end{array}$

$\begin{array}{lll}4.6 & \text { Marktpositionierung } & 19\end{array}$

5 Die Einschätzung des Förderprogramms 21

5.1 Bekanntheit des Programms und Kontaktaufnahme 21

$\begin{array}{lll}5.2 & \text { Die Rolle von Kooperationen } & 22\end{array}$

5.3 Bedeutung der Programmteilnahme: Ziele und Zielerreichung 24

$\begin{array}{lll}5.4 & \text { Schwierigkeiten im Projektverlauf } & 27\end{array}$

5.5 Kommerzielle Verwertung und Fortsetzung der Forschungstätigkeiten 29

5.6 Kosten und Nutzen der Programmteilnahme 30

5.7 Bewertung der administrativen Programmabwicklung 31

6 Additionalitäten $\quad 32$

$\begin{array}{lll}\text { 6.1 } & \text { Fragebogenauswertung und Interviews } & 34\end{array}$

6.2 Ökonometrische Analyse $\quad 35$

7 Volkswirtschaftliche und außenwirtschaftliche Wirkungen 39

$\begin{array}{lll}7.1 & \text { Fragestellung } & 39\end{array}$

7.3 Forschungsförderung und Dynamik der Holzwirtschaft in den Jahren 1997 bis 2000

7.4 Der Außenhandel mit Holz und Holzprodukten 46

7.5 Forschungsförderung und internationale Wettbewerbsfähigkeit 52

8 Schlussfolgerungen $\quad 54$

9 Literaturverzeichnis $\quad 62$

$\begin{array}{lll}\text { Appendix: } & \text { Tabellen und Abbildungen } & 65\end{array}$ 


\section{Tabellenverzeichnis}

Tabelle 1:

Tabelle 2:

Tabelle 3:

Tabelle 4:

Tabelle 5:

Tabelle 6:

Tabelle 7:

Tabelle 8:

Tabelle 9:

Tabelle 10:

Tabelle 11:

Tabelle 12:

Tabelle 13:

Tabelle 14:

Tabelle 15:

Tabelle 16:

Tabelle 17:

Tabelle 18:

Tabelle 19:

Tabelle 20:

Tabelle 21:

Tabelle 22:

Tabelle 23:

Tabelle 24.

Tabelle 25:

Tabelle 26:

Tabelle 27:

Tabelle 28:

Tabelle 29:

Tabelle 30:

Tabelle 28:

Tabelle 29:

Tabelle 30:

Tabelle 31:

Tabelle 32:

Tabelle 33:

Tabelle 34:

Tabelle 35:

Tabelle 36:

Tabelle 37:

Tabelle 38:

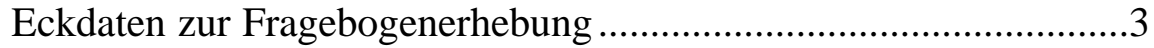

Eckdaten zur Sonderaktion Holzforschung ......................................

Aufteilung nach technischen Bereichen.........................................6

Aufteilung der geförderten Projekte auf die Bundesländer [\%] .........7

Projektbewertung anhand der FFF Auswahlkriterien .......................8

Ergebnisse logistische Regression ................................................9

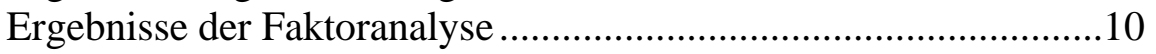

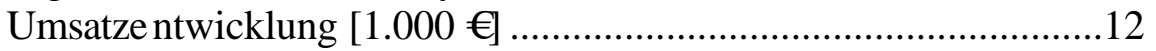

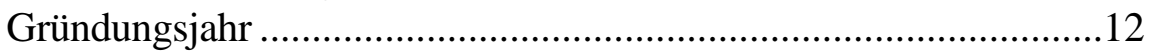

Durchschnittliche Anzahl an Mitarbeitern und Umsatz pro

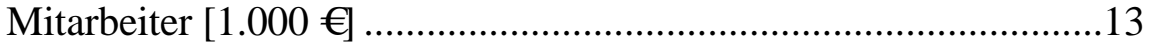

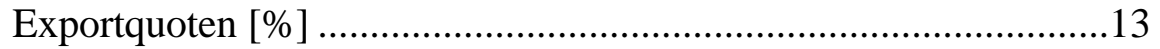

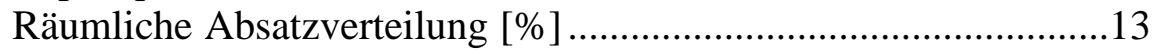

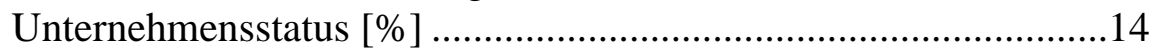

Trennung von Eigentum und Management [\%] ..............................14

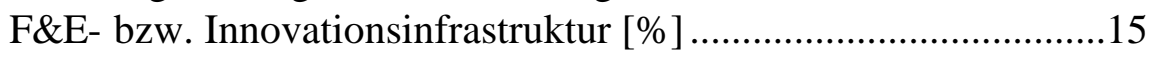

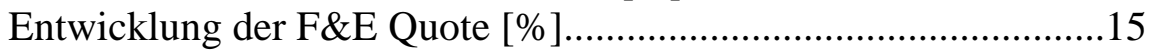

Bildungsabschluss der Mitarbeiter [\%] ........................................16

Entwicklung der Patentanmeldungen [je 1.000 Mitarbeiter] ..........16

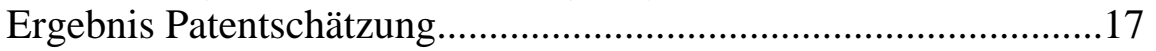

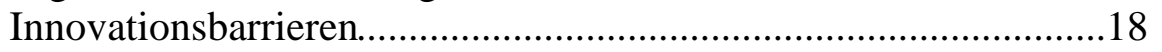

Kooperationspartner im Bereich Holzforschung [\%] ......................18

Kategorisierung anhand des wichtigsten Produkts [\%]....................19

Entwicklung des Preisdrucks [\%] ...............................................19

Eigene Stärken und Wichtigkeit für Wettbewerbsfähigkeit............20

Wie wurden die Förderteilnehmer auf die Sonderaktion

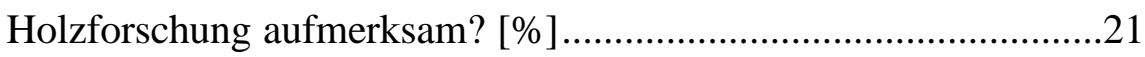

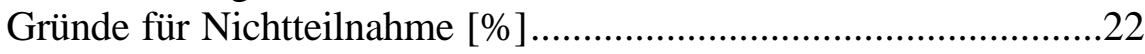

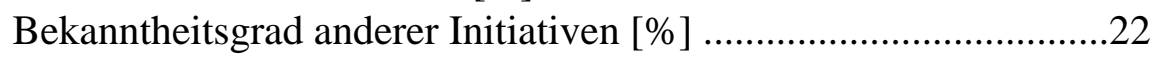

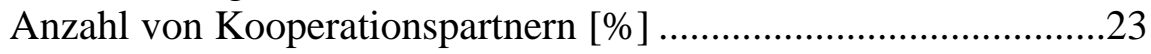

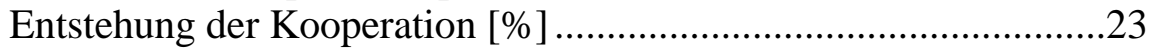

Struktur der Kooperationen [\%] ...................................................24

Bedeutende Ziele und deren Erreichung [absolute Häufigkeiten] ..26

Mittlere Zielerreichung [Median] bei bedeutsamen und

unbedeutsamen Zielsetzungen. .27

Im Rahmen des Projektverlaufs eingetretene Schwierigkeiten und deren Lösung [absolute Häufigkeiten] .............................................28

Kommerzielle Verwertung der Ergebnisse [\%] ..............................29

Zeitlicher Abstand zwischen Projektbeginn und kommerzieller

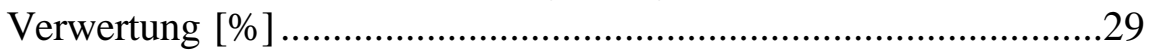

Pläne nach Projektende [\%] ...........................................................30

Kosten-Nutzen-Einschätzung der Programmteilnahme durch die

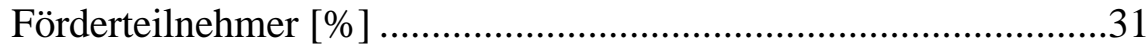

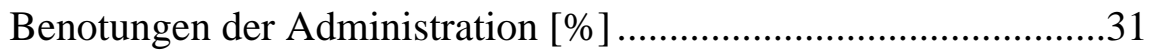

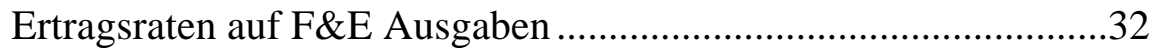

Additionalität des Programms [\%] .................................................34

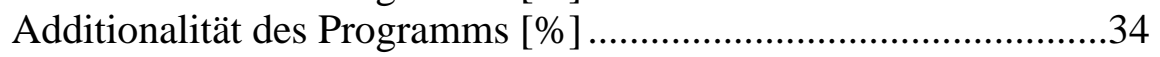


Tabelle 39: $\quad$ Einfluss von F\&E-Subventionen- Studien auf Firmenebene ...........35

Tabelle 40: $\quad$ Regressionsergebnisse der GMM Schätzung.................................37

Tabelle 41: Außenwirtschaftskennzahlen [1995 und 2000].............................47

Tabelle 42: $\quad$ Veränderung der internationalen Wettbewerbsposition der österreichischen Holzwirtschaft [1997 - 2000] ..............................52

Tabelle 43: $\quad$ Forschungsförderung und Exporterfolge …...................................53

Tabelle 44: $\quad$ Kennzahlen der Holzwirtschaft [1995] .......................................65

Tabelle 45: $\quad$ Entwicklung der Kennzahlen der Holzwirtschaft [1995/2000].......66

Tabelle 46: $\quad$ Forschungsförderung und Wachstum in der Holzwirtschaft ...........67

Tabelle 47: $\quad$ Österreichischer Außenhandel mit Holz und Holzprodukten [1998

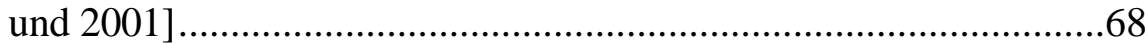

Tabelle 48: $\quad$ Die wichtigsten Importeure von Rohholz, einfach bearbeitetem Holz und Furnieren [2000] ..........................................................69

Tabelle 49: $\quad$ Die wichtigsten Importeure von Holzplatten [2000]........................70

Tabelle 50: Die wichtigsten Importeure von Verpackungen und Bauwaren aus Holz [2000] ................................................................................. 71

Tabelle 51: Die wichtigsten Importeure von vorgefertigten Häusern und

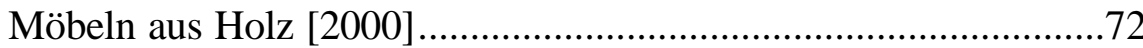




\section{Abbildungsverzeichnis}

Abbildung 1:

Abbildung 2:

Abbildung 3:

Abbildung 4:

Abbildung 5:

Abbildung 6:

Abbildung 7:

Abbildung 8:

Abbildung 9:

Abbildung 10:

Abbildung 11:

Abbildung 12:

Abbildung 13:

Abbildung 14:

Abbildung 15:

Abbildung 16:

Abbildung 17:

Abbildung 18:

Abbildung 19:

Abbildung 20:

Abbildung 21:

Abbildung 22:

Abbildung 23:

Abbildung 24:

Abbildung 25:

Abbildung 26:

Abbildung 27:

Abbildung 28:

Abbildung 29:

Abbildung 30:

Abbildung 31:

Abbildung 32:

Abbildung 33:

Abbildung 34:

Abbildung 35:

Abbildung 36:

Abbildung 37:

Abbildung 38:

Abbildung 39:
Kumulierte Häufigkeitsverteilung der Förderbarwerte [1.000 €] .....4

Entwicklung des Produktionswertes in der Holzwirtschaft [1995 =

100]. 41

Entwicklung der Beschäftigten in der Holzwirtschaft [1995=100] 42

Entwicklung der Wertschöpfung je Beschäftigten in der

Holzwirtschaft $[1995=100]$ .43

Entwicklung des Personalaufwandes je unselbständig

Beschäftigten in der Holzwirtschaft [1995 = 100] .44

Holzwaren, roh und einfach behandelt, Index 1998=100 [1990 2001]..... . .73

Export von Holzwaren, roh und einfach behandelt, Unit Values

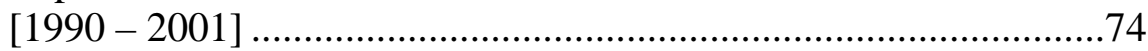

Holzwaren, roh und einfach behandelt, RCA-Werte [1990 - 2001]75

Exportwert von Furnieren und Platten aus Holz, Index $1998=100$

[1990 - 2001] .76

Export von Furnieren und Platten aus Holz, Unit Values [1990 -

2001]..... .77

Furniere und Platten aus Holz, RCA-Werte [1990 - 2001] .............78

Exportwert von Holzwaren, Index $1998=100$ [1990 - 2001] .......79

Export von Holzwaren, Unit Values [1990 - 2001] .........................80

Holzwaren, RCA-Werte [1990 - 2001] ........................................81

Exportwert von Möbeln aus Holz [1990 - 2001] ............................82

Export von Möbeln aus Holz, Unit Values [1990 - 2001]...............83

Möbel aus Holz, RCA-Werte [1990 - 2001] ...................................84

Dynamik der Exporte von Nadelrohholz, unbehandelt .....................85

Dynamik der Exporte von anderem Rohholz, unbehandelt ..............86

Dynamik der Exporte von Nadelholz ges. >6mm ...........................87

Dynamik der Exporte von Nadelholz profiliert................................88

Dynamik der Exporte von anderem Holz ges. $>6 \mathrm{~mm}$.......................89

Dynamik der Exporte von anderem Holz profiliert .........................90

Dynamik der Exporte von Furnieren aus Nadelholz.......................90

Dynamik der Exporte von Furnieren aus anderem Holz..................91

Dynamik der Exporte von Span- u.a. Platten aus Holz....................92

Dynamik der Exporte von Sperrholz mit Tropenholz.....................93

Dynamik der Exporte von anderem Sperrholz oder Furnier...........94

Dynamik der Exporte von and. Sperrholz aus Nadelholz ...............95

Dynamik der Exporte von anderem Sperrholz u.ä. a.n.g. ...............96

Dynamik der Exporte von Faserplatten > 0,8 g/cm3 ......................97

Dynamik der Exporte von Faserplatten 0,5 - 0,8 g/cm3 .................98

Dynamik der Exporte von Faserplatten 0,35 - 0,5 g/cm3 ...............99

Dynamik der Exporte von Kisten, Trommeln u.ä. ........................100

Dynamik der Exporte von Flachpaletten u.dgl...............................101

Dynamik der Exporte von Fässern, Trögen u.dgl. .........................102

Dynamik der Exporte von Holzfenstern u.dgl. ............................103

Dynamik der Exporte von Holztüren u.dgl. ...................................104

Dynamik der Exporte von anderen Bautischlerarbeiten (darunter

Konstruktionsholz)..... 105 
Abbildung 40: Dynamik der Exporte von vorgefertigten Häusern........................106

Abbildung 41: Dynamik der Exporte von Sitzen a.n.g. mit Holzgestell .................107

Abbildung 42: Dynamik der Exporte von Büromöbeln aus Holz ..........................108

Abbildung 43: Dynamik der Exporte von Küchenmöbeln aus Holz......................109

Abbildung 44: Dynamik der Exporte von Schlafzimmermöbeln aus Holz............110

Abbildung 45: Dynamik der Exporte von anderen Möbeln aus Holz ....................111 


\section{$1 \quad$ Einleitung}

Mit der Durchführung der Sonderaktion Holzforschung über die Jahre 1996-1999 hat der FFF eine Reihe von Defiziten des österreichischen Innovationssystems im Allgemeinen und der österreichischen Holzwirtschaft im Besonderen ange sprochen:

- Generell niedriges F\&E-Niveau der österreichischen Unternehmen, vor allem auch in der Holzwirtschaft. Im internationalen Vergleich hinkt Österreich insbesondere im Anteil der privaten F\&E-Ausgaben hinterher.

- Fragmentierte Landschaft an innovationsunterstützenden Einrichtungen, abermals auch im Bereich der Holzwirtschaft. Die Befunde dazu zeigen eine geringe Koordination zwischen den Forschungsstätten, auf unterkritische Kapazitäten und ein unübersichtliches Leistungsangebot.

- Rohstofflastigkeit und geringe Wertschöpfungstiefe der österreichischen Holzwirtschaft mit dementsprechend hoher Konjunkturabhängigkeit und Gefährdung im europäischen Kostenwettbewerb.

- Geringe sektorübergreifende Kooperationsbereitschaft bei den überwiegend mittelständischen Unternehmen.

Vor diesem Hintergrund verfolgte die Sonderaktion Holzforschung eine Reihe von Zielen.

- Verbesserung der Forschungssituation

- höhere Wertschöpfung

- Stärkung der Forschungskapazität

- Beseitigung von Innovationshemmnissen

- nationale und internationale Kooperationen

- Steigerung der Projekte

- insgesamt: effiziente Holzforschung

Im Zuge der Zwischenevaluierung (Ohler, Teischinger, Schörghofer 1998), die eine Fortsetzung des Programms empfohlen hat, wurde zugleich vorgeschlagen, die Zielsetzungen des Programms einerseits zu präzisieren und sie andererseits in ihrem Spektrum einzuschränken. Im Besonderen wurde vorgeschlagen, beim Kooperationsaspekt deutlicher zwischen unterschiedlichen Formen der Kooperation und deren Bedeutung für das Projekt bzw. die beteiligten Kooperationspartner zu unterscheiden.

Die Zwischenevaluierung selbst wurde in der Weise durchgeführt, dass ein Dreierteam mit Kompetenzen auf dem Gebiet der Förderung, der Evaluierung und des Holzsektors - jedes einzelne Projekt bewertet ${ }^{1}$ hat. Als Informationsgrundlage galten das Förderansuchen und die beiden Gutachten durch den FFF. Diese Methode, bei der die Förderwerber nicht involviert wurden, wurde vor allem deshalb gewählt, um

Die Bewertung der Projekte erfolgte nach folgenden Kriterien: (i) technologische Qualität der Projekte, (ii) Verbesserung der Forschungssituation, (iii) Intelligentes Portfolio / Wertschöpfung, (iv) Kooperationsqualität und (v) externe Effekte. Diese Kriterien wurden für den praktischen Gebrauch verfeinert und operationalisiert und mit den generellen Zielsetzungen abgestimmt. 
die Bereitschaft der Förderwerber für eine Fragebogenerhebung zur Verfügung zu stellen, nicht zu 'verheizen', zumal absehbar war, dass eine ex-post Evaluierung in jedem Fall durchgeführt werden sollte.

Diese ex-post Evaluierung liegt mit diesem Bericht nunmehr vor und umfasst folgende Analysen:

In Kapitel 2 wird die Untersuchungsmethode der Evaluierung vorgestellt und ein Überblick über die verwendeten Datensatz gegeben, der sowohl Programmteilnehmer als auch eine Kontrollgruppe umfasst.

Kapitel 3 beschreibt die Sonderaktion Holzforschung anhand wichtiger Kennziffern wie Fördervolumen und Förderquote. In diesem Kapitel wird außerdem das Fördervergabeverfahren beschrieben und ausgewertet.

In Kapitel 4 werden die teilnehmenden Firmen charakterisiert und der Kontrollgruppe gegenübergestellt. Der Vergleich wird anhand wichtiger Indikatoren wie Umsatz, Wachstum, Export- und insbesondere FuE-Aktivitäten vorgenommen.

Nachdem der Leser ein Bild über die Adressaten des Förderprogramms gewonnen hat, behandelt Kapitel 5 die Einschätzungen der Sonderaktion Holzforschung aus Sicht der Programmteilnehmer. Dies ist insofern ein Kernkapitel der Evaluierung, als dass hier entscheidende Fragen folgender Art behandelt werden: Welche Ziele haben die Unternehmen mit der Teilnahme verknüpft? In welchem Maße wurden diese Ziele erreicht? Hat der Nutzen der Teilnahme die diesbezüglichen Kosten überwogen? Außerdem wird in diesem Kapitel die administrative Programmabwicklung von den Teilnehmern benotet.

Kapitel 6 widmet sich anschließend einer Spezialfrage, nämlich den Programmadditionalitäten. Hier wird versucht zu eruieren, welche Nettowirkungen das Programm entfalten konnte, indem die Förderungen in eine kausale Beziehung zu den FuEInvestitionen gesetzt werden.

In Kapitel 7 wird der Blickwinkel von der Firmenebene auf die volks- und außenwirtschaftliche Ebene gewechselt. Es wird anhand offizieller Statistiken ein Bild der österreichischen Holzwirtschaft gezeichnet und versucht, die Entwicklungen der letzten Jahre mit der Sonderaktion Holzforschung in Beziehung zu setzen.

Kapitel 8 liefert eine abschließende Zusammenfassung der wichtigsten Ergebnisse. Es soll hier auch über den Anlass hinaus geblickt werden, um für andere, ähnlich gelagerte Förderprogramme, Lektionen zu erschließen. 


\section{Methode}

Die Evaluierung stützt sich auf drei Säulen: (i) Förderdatenbank des FFF, (ii) Fragebogenerhebung und (iii) persönliche Interviews mit Repräsentanten von geförderten Programmteilnehmern.

Im Rahmen der Fragebogenerhebung haben der FFF und Technopolis insgesamt 384 Fragebögen an Unternehmen aus der Holzindustrie verschickt. Der FFF hat aus Anonymitätsgründen die Fragebögen an 148 Teilnehmer der Sonderaktion und an 33 abgelehnte Bewerber gesandt. Bei den Teilnehmern betrug die Rücklaufquote nach zwei Nachfassaktionen 52\%, was im Rahmen der Erwartungen lag. Die Kontrollgruppe rekrutiert sich aus Firmen, die weder an der Sonderaktion teilgenommen, noch sich dafür beworben haben. Das Mitgliedsverzeichnis des Fachverbandes der Holzindustrie diente zur Identifizierung von Kontaktadressen. Erwartungsgemäß war der Rücklauf bei der Kontrollgruppe deutlich niedriger als bei den Teilnehmern. Nur 27 von 203 angeschriebenen Unternehmen returnierten den Fragebogen. Einen tabellarischen Überblick der Fragebogenerhebung liefert Tabelle 1.

Tabelle 1: $\quad$ Eckdaten zur Fragebogenerhebung

\begin{tabular}{lrrrr} 
& $\begin{array}{r}\text { Daten vom } \\
\text { FFF }\end{array}$ & $\begin{array}{c}\text { Daten vom } \\
\text { Fachverband }\end{array}$ & $\begin{array}{r}\text { retournierte } \\
\text { Fragebögen }\end{array}$ & $\begin{array}{r}\text { Rücklaufquote } \\
{[\%]}\end{array}$ \\
\hline Förderteilnehmer & 148 & & 77 & 52 \\
abgelehnte Bewerber & 33 & 203 & 5 & 15 \\
Kontrollgruppe & & 203 & 27 & 13 \\
\hline insgesamt & 181 & & &
\end{tabular}

Durch die returnierten Fragebögen werden 160 der 232 geförderten Projekte erfasst. Bei den abgelehnten sind dies 14 von 47. Die Daten aus der Fragebogenerhebung wurden mit der Förderdatenbank vom FFF verschmolzen, in der die Unternehmenund Projektdaten der Antragssteller gespeichert sind. Durch die Fusion der beiden Datensätze wurde eine Zeitreihe aufgebaut, mit deren Hilfe sich robuste Auswertungen machen lassen.

Da rein quantitative Evaluierungsansätze zwangsläufig zu kurz greifen, wenn es darum geht, die Dynamik des Innovationsgeschehens im Unternehmen nachzuzeichnen, wurden ergänzend Interviews mit teilnehmenden Firmen durchgeführt, um Innovationsbarrieren, Erfolgsfaktoren und Motivlagen der Firmen besser zu verstehen. 


\section{Eckdaten des Programms}

\subsection{Zahl der Förderwerber, Förderquote}

Im Rahmen der Sonderaktion Holzforschung wurden in Zeitraum 1996 bis 1999232 Projekte von 148 Unternehmen gefördert. 47 weitere Einreichungen von 33 Firmen sind abgelehnt worden. Die gesamten Förderungen hatten ein Volumen von 14,5 Mio. €. Diese Summe ist als Barwert zu verstehen, der sich aus der Summe aus den direkten Zuschüssen und dem Zinsvorteil gegenüber banküblichen Konditionen bei Darlehen ergibt. Im Schnitt wurde ein Projekt mit ca. $63.000 €$ unterstützt. Die Streuung ist dabei allerdings groß. Während das kleinste Projekt eine Unterstützung von $7.500 €$ erfuhr, wurde das größte mit knapp $400.000 €$ gefördert. Das bedeutet, dass die 20 höchsten Förderungen (das sind 8,6\% der Projekte) bereits 3,5 Mio. €, also $25 \%$ der Gesamtförderung, ausmachen (Abbildung 1).

\section{Abbildung 1: Kumulierte Häufigkeitsverteilung der Förderbarwerte [1.000 € ]}

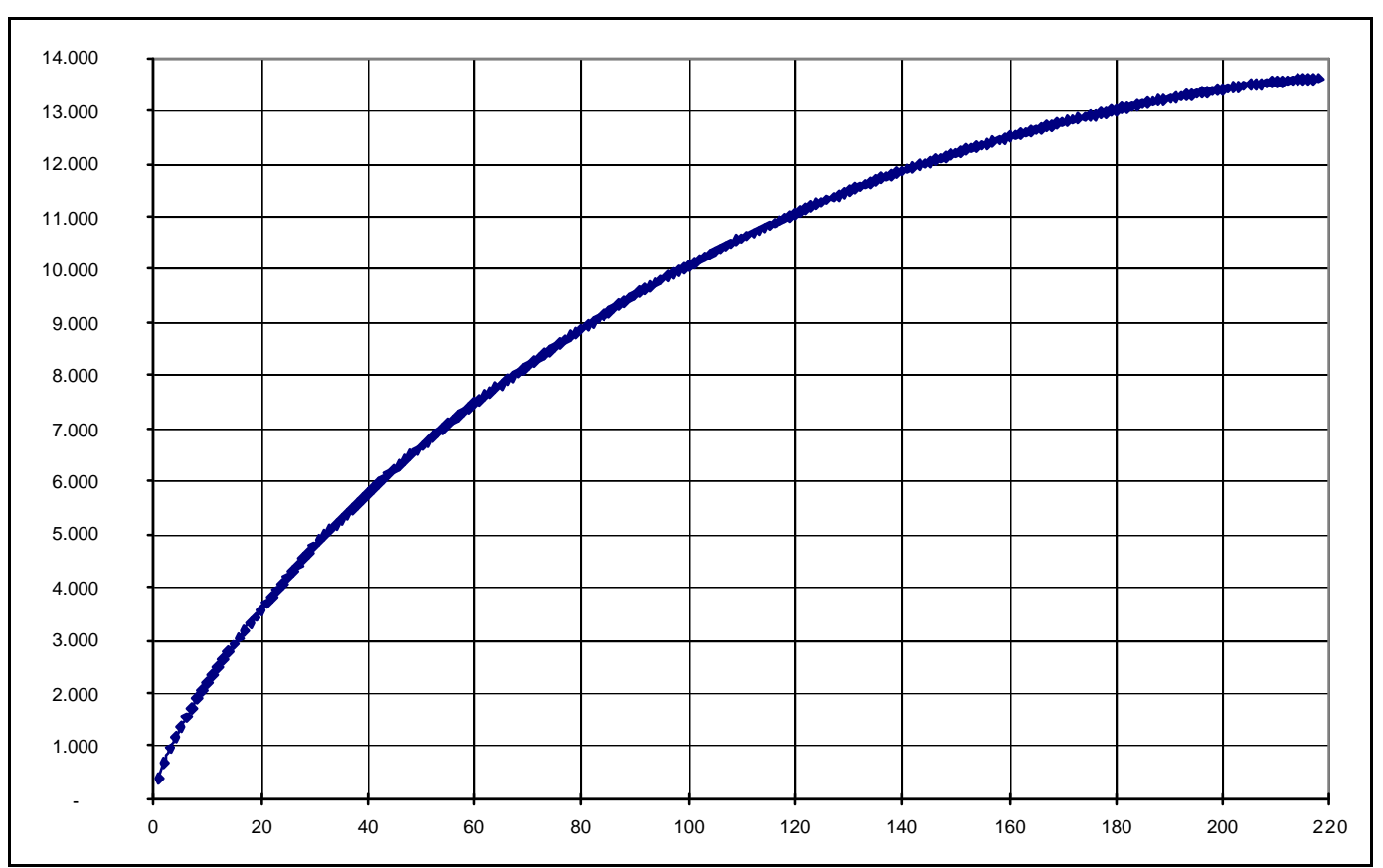

Quelle: FFF, eigene Berechnungen

Mit den 14,5 Mio. € Förderungen wurden Projekte mit einem Volumen von 57 Mio. $€$ unterstützt, die durchschnittliche rund betrug also 25\%. Die 57 Mio. $€$ Projektkosten sind die vom FFF akzeptierten, sie liegen deutlich niedriger als die vom Teilnehmer bei der Einreichung veranschlagten Ausgaben, die sich auf über 78 Mio. $€$ beliefen. Legt man die Kostenschätzungen der Teilnehmer zugrunde, so kommt man auf eine Förderquote von unter 20\%. Die maximale Förderhöhe, die laut Richtlinien des FFF möglich ist, beträgt 50\% der Projektkosten. Die Diskrepanz zwischen den FFF Projektkostenschätzungen und denen des Unternehmens ist bei den abgelehnten Firmen besonders augenfällig. Wenn die Kostenschätzungen des FFF der Realität entsprechen - woran nicht gezweifelt wird - liegt der Verdacht nahe, dass sich unter den abgelehnten Unternehmen einige schwarze Schafe befanden, die sich durch die Teilnahme an der Sonderaktion finanziell sanieren wollten. 
Von den drei Kostenarten Personalkosten, Einrichtungskosten und sonstige Kosten wurden übrigens die meisten Abstriche bei den Personalkosten gemacht. Im Schnitt wurden hier knapp über 50\% akzeptiert. Bei den Einrichtungskosten hingegen lag der Satz bei $73 \%$ und bei den sonstigen Kosten bei über $94 \%$. Warum ist die Lücke bei den Personalkosten höher als bei den anderen Kostenarten? Drei Erklärungen bieten sich an: (i) Sachkosten sind leichter zu überprüfen und daher sind Unternehmen in diesem Bereich ehrlicher. Bei den schwerer zu überprüfenden Personalkosten hingegen ist man versucht, die Kosten aufzubauschen. (ii) Die Unternehmen sind zwar auch bei den Personalkosten ehrlich, der FFF sieht jedoch entweder Spielräume bei der Arbeitsproduktivität oder hat eine andere Auffassung darüber, welche Arbeit noch dem Projekt zugerechnet werden kann. Erfahrungsgemäß planen viele Förderwerber eine größere Lernphase vor Projektbeginn bzw. eine Nachberarbeitungsphase am Ende des Projektes ein, wodurch eher der zweiten Interpretation der Vorzug zu geben ist. (iii) Laut Angaben des FFF resultiert eine weitere Diskrepanz bei den Personalkosten daraus, dass die Unternehmen versuchen, die Personalkosten geltend zu machen, die sie am Markt verrechnen und nicht die Selbstkosten, die dem Unternehmen wirklich anfallen.

Tabelle 2: $\quad$ Eckdaten zur Sonderaktion Holzforschung

\begin{tabular}{lrr}
\hline & Teilnehmer & Abgelehnte \\
\hline Projekte & 232 & 47 \\
Firmen & 148 & 33 \\
Förderung/Projekt $\varnothing[1.000 €]$ & 63 & - \\
Projektvolumen $\varnothing[1.000 €]$ & 260 & 110 \\
eingereichte Projektkosten S [Mio. €] & 78 & 12 \\
anerkannte Projektkosten S [Mio. €] & 57 & 3,8 \\
Förderquote $\varnothing[\%]$ & 25 & - \\
Förderung Total [Mio. $€]$ & 14,5 & - \\
\hline
\end{tabular}

Quelle: FFF, eigene Berechnungen

Wir haben außerdem überprüft, ob es einen Zusammenhang zwischen dem Förderbarwert und der Förderquote gibt und haben hier keine signifikante Beziehung gefunden. Aus zweierlei Gründen hätte man hier einen Zusammenhang erwarten können. Einerseits kann man oft beobachten, dass größere absolute Fördervolumina auch mit einer höheren Förderquote einhergehen, da größere Forschungsprojekte meist von größeren Firmen durchgeführt werden, die bereits Erfahrung beim Einwerben von Fördermitteln haben und daher die Förderquote beeinflussen können. Dies scheint hier nicht der Fall zu sein und wird positiv gewertet. Auf der anderen Seite wäre es möglich, dass kleinere Projekte eher von Einsteigern gemacht werden, die einen Einsteigerbonus von der Förderinstitution erhalten. Auch dies trifft nicht zu, weshalb man das Fazit ziehen kann, dass der FFF primär die Projekte bewertet und hier ein hohes Maß an Objektivität walten lässt und sich nicht von äußeren Umständen leiten lässt.

\subsection{Verteilung nach Branchen}

Schlüsselt man die Verteilung nach Branchenkategorien auf, so entfielen rund 55\% auf Projekte der Holzwirtschaft im engeren Sinne (Holzbearbeitung und Holzverarbeitung, Möbelerzeugung). Der Rest verteilt sich auf Branchen, die mit der Holzwirtschaft verbunden sind, vor allem Forstwirtschaft, Maschinenbau und chemische 
Industrie. Den größten Anteil an der Forschungsförderung hatten mit 21\% die Entwickler von Bearbeitungstechnologien für die Holzwirtschaft (Tabelle 3).

Tabelle 3: Aufteilung nach technischen Bereichen

\begin{tabular}{lrr}
\hline & Barwert [1.000 $€]$ & {$[\%]$} \\
\hline Bearbeitungstechnologien & 3.017 & 20,8 \\
Konstruktiver Holzbau & 2.656 & 18,3 \\
Platten & 1.602 & 11,0 \\
Forst & 1.049 & 7,2 \\
Oberflächenschutz, Leim & 1.019 & 7,0 \\
Trocknung & 766 & 5,3 \\
Möbel & 744 & 5,1 \\
Qualitätskontrolle & 684 & 4,7 \\
Innenausbau & 618 & 4,3 \\
Einschnitt & 469 & 3,2 \\
Türen, Fenster & 433 & 3,0 \\
Altholzverwertung & 274 & 1,9 \\
Schalungselemente & 176 & 1,2 \\
Sonstige Produkte & 999 & 6,9 \\
\hline Insgesamt & 14.506 & 100,0 \\
\hline Qutle:
\end{tabular}

Quelle: FFF, eigene Berechnungen

Es handelt sich dabei um ein Gruppe von Förderempfängern, die im Sinne der Branchengliederung nicht dem Holzsektor zuzurechnen sind. Das heißt, dass 1/3 der Empfänger nicht dem Holzsektor im engeren Sinne angehört. Im Lichte der Diskussion von Industrieclustern lässt sich hier sehr deutlich ein Clusteransatz in der Förderphilosophie beobachten

\subsection{Verteilung nach Bundesländern}

Die regionale Verteilung der Förderungen - auch im Vergleich mit der Umsatzverteilung der österreichischen Holzwirtschaft insgesamt - ist in Tabelle 4 dargestellt. ${ }^{2}$ Oberösterreich konnte die relative Mehrheit der Förderungen akquirieren. Der Förderanteil lag hier deutlich über dem Anteil an der Holzwirtschaft insgesamt ${ }^{3}$. Während bei vielen Förderprogrammen der Großraum Wien überrepräsentiert ist, ist dies hier offenbar nicht der Fall, was strukturpolitisch durchaus positiv zu bewerten ist.

2 Unter Holzwirtschaft ist hier die NACE Kategorie 20 'Be- und Verarbeitung von Holz' gemeint.

3 Dies ist übrigens ein Hinweis darauf, dass sich die bisweilen behauptete 'Abwerbung' von Förderwerbern aufgrund regionaler Clusterinitiativen hier jedenfalls nicht feststellen lässt. 
Tabelle 4: $\quad$ Aufteilung der geförderten Projekte auf die Bundesländer [\%]

\begin{tabular}{lrr}
\hline & Anteil Holzindustrie & Anteil Förderungen \\
\hline Wien & 2,99 & 4,2 \\
Burgenland & 2,52 & 4,4 \\
Vorarlberg & 4,35 & 5,9 \\
Tirol & 11,3 & 7,7 \\
Salzburg & 11,8 & 11,8 \\
Steiermark & 17,7 & 12,6 \\
Niederösterreich & 20,2 & 14,8 \\
Kärnten & 10,7 & 15,4 \\
Oberösterreich & 18,1 & 22,8 \\
\hline
\end{tabular}

Quelle: Statistik Austria, FFF, eigene Berechnung

\subsection{Treffsicherheit der Förderkriterien und Projektbewertung}

Werfen wir abschließend noch einen Blick auf die Bewertungskriterien des FFF. Jeder beim FFF eingehende Antrag auf Förderung wird anhand einer Checkliste bewertet, die sowohl technologische als auch wirtschaftliche Aspekte beinhaltet (Tabelle 5). Insgesamt sollte ein Projektvorschlag in allen vier relevanten Teilaspekten (technische Bewertung von Firma und Projekt, sowie wirtschaftliche Bewertung von Firma und Markt) jeweils mehr als 20 von 50 möglichen Punkte sammeln.

Die vier Teilaspekte sind wiederum in insgesamt 14 verschiedene Unterkriterien aufgegliedert, ${ }^{4}$ die jeweils individuell gewichtet werden. Der Gewichtungsfaktor drückt sich in der Höhe der maximal erreichbaren Punktzahl aus. Das wichtigste Einzelkriterium ist hier die finanzielle Leistungsfähigkeit des Unternehmens, bei dem der Antragsteller bis zu 30 Punkte sammeln kann. Dies ist ein etwas zweischneidiger Punkt. Einerseits ist es nachvollziehbar, dass sich der FFF finanzstarke Partner wünscht, bei denen die Ausfallwahrscheinlichkeit gering ist. Andererseits stellt dieser Punkt eine gewisse Hürde für kleine 'start-up' Unternehmen dar, also Firmen, die gerade wegen ihrer geringeren Finanzkraft F\&E-Projekte nicht durchstehen können und insofern förderungsbedürftig sind.

Alle Kriterien bis auf den Punkt 'Umwelt', der allerdings einen eher niedrigen Stellenwert hat, differenzieren signifikant zwischen abgelehnten und akzeptierten Bewerbern. Die größten relativen Unterschiede gibt es bei den grau markierten Themenbereichen, die mit der Marktsituation zu tun haben. Die Tatsache, dass der Benotungsunterschied bei der Markterfahrung am Größten ist, spricht wieder dafür, dass es Neugründungen hier eher schwer haben. Laut Angaben des FFF scheitern allerdings die meisten Projekte nicht an technischen Hürden, sondern an der fehlenden Fähigkeit der Unternehmen, die für die neu entwickelten Produkte oder Prozesse auch einen Markt zu entwickeln. Insofern sei es zu rechtfertigen, dass die marktrelevanten Punkte einen hohen Stellenwert haben.

4 Beispielsweise setzt sich der Teilaspekt 'technische Kriterien-Bewertung Firma' aus den Unterkriterien 'Durchführbarkeit', 'F\&E-Dynamik', und 'Know How Zuwachs' zusammen. 
Tabelle 5: $\quad$ Projektbewertung anhand der FFF Auswahlkriterien

\begin{tabular}{|c|c|c|c|c|c|c|c|}
\hline & & & Projektstatus & $\begin{array}{r}\text { Max. } \\
\text { Punkte }\end{array}$ & $\begin{array}{r}\varnothing \\
\text { Punkte }\end{array}$ & $\begin{array}{r}\text { absoluter } \\
\text { Abstand }\end{array}$ & $\begin{array}{l}\text { relativer } \\
\text { Abstand }\end{array}$ \\
\hline \multirow{18}{*}{ 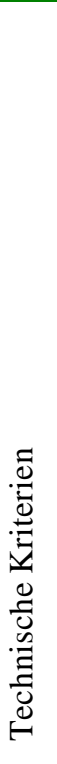 } & \multirow{8}{*}{ 节 } & Durchführbarkeit & bewilligt & \multirow{2}{*}{15} & 9,2 & \multirow{2}{*}{3,7} & \multirow{2}{*}{$68,4 \%$} \\
\hline & & & abgelehnt & & 5,5 & & \\
\hline & & F\&E-Dynamik & bewilligt & \multirow{2}{*}{10} & 6,8 & \multirow{2}{*}{2,3} & \multirow{2}{*}{$49,9 \%$} \\
\hline & & & abgelehnt & & 4,6 & & \\
\hline & & Know How Zuwachs & bewilligt & \multirow{2}{*}{25} & 14,3 & \multirow{2}{*}{5,5} & \multirow{2}{*}{$62,5 \%$} \\
\hline & & & abgelehnt & & 8,8 & & \\
\hline & & Bewertung Firma & bewilligt & \multirow{2}{*}{50} & 30,0 & \multirow{2}{*}{11,6} & \multirow{2}{*}{$63,1 \%$} \\
\hline & & & abgelehnt & & 18,4 & & \\
\hline & \multirow{10}{*}{$\begin{array}{l}\frac{\pi}{0} \\
\frac{0}{0} \\
0\end{array}$} & Umwelt & bewilligt & \multirow{2}{*}{5} & 2,5 & \multirow{2}{*}{0,5} & \multirow{2}{*}{$22,1 \%$} \\
\hline & & & abgelehnt & & 2,0 & & \\
\hline & & Nutzen & bewilligt & \multirow{2}{*}{10} & 5,9 & \multirow{2}{*}{2,6} & \multirow{2}{*}{$77,4 \%$} \\
\hline & & & abgelehnt & & 3,4 & & \\
\hline & & Risiko & bewilligt & \multirow{2}{*}{20} & 11,6 & \multirow{2}{*}{4,1} & \multirow{2}{*}{$54,3 \%$} \\
\hline & & & abgelehnt & & 7,5 & & \\
\hline & & Neuheit & bewilligt & \multirow{2}{*}{15} & 9,8 & 30 & $674 \%$ \\
\hline & & & abgelehnt & & 5,8 & 3,9 & $0 /, 4 \%$ \\
\hline & & Bewertung Projekt & bewilligt & 50 & 29,7 & 125 & $729 \%$ \\
\hline & & & abgelehnt & 50 & 17,2 & 12,5 & $12,9 \%$ \\
\hline & & Soziale Aspekte & bewilligt & & 2,1 & & \\
\hline & & & abgelehnt & 5 & 1,3 & 0,8 & $6 /, / \%$ \\
\hline & & Externe Effekte & bewilligt & 10 & 2,4 & 10 & $702 \%$ \\
\hline & & & abgelehnt & 10 & 1,4 & 1,0 & $10,2 \%$ \\
\hline & & Verwertung & bewilligt & 10 & 5,7 & 29 & $1052 \%$ \\
\hline & & & abgelehnt & 10 & 2,8 & 2,9 & $105,2 \%$ \\
\hline & & Marktaussichten & bewilligt & 15 & 10,1 & 52 & $1081 \%$ \\
\hline & & & abgelehnt & 15 & 4,8 & 3,2 & $100,1 \%$ \\
\hline & & Markterfahrung & bewilligt & 10 & 7,3 & 44 & $1543 \%$ \\
\hline & & & abgelehnt & 10 & 2,9 & 4,4 & $154,5 \%$ \\
\hline & 茫 & Marktsituation & bewilligt & & 27,1 & & \\
\hline$\cdot \frac{\mathscr{O}}{0}$ & $\Sigma$ & & abgelehnt & 50 & 13,5 & 13,6 & $101,2 \%$ \\
\hline 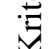 & & Management & bewilligt & 20 & 5,6 & 18 & $483 \%$ \\
\hline d & & & abgelehnt & 20 & 3,8 & 1,8 & $48,3 \%$ \\
\hline . & & $\begin{array}{l}\text { Finanzielle } \\
\text { Leistungsfähigkeit }\end{array}$ & bewilligt & 30 & 14,5 & 5,8 & $67,3 \%$ \\
\hline ల్ & & & abgelehnt & & 8,6 & & \\
\hline$\stackrel{=}{=}$ & $\underline{G}$ & Firmensituation & bewilligt & 50 & 20,2 & 77 & $621 \%$ \\
\hline 3 & ⿷匚 & & abgelehnt & 50 & 12,4 & 1,1 & $02,1 \%$ \\
\hline
\end{tabular}

Quelle: FFF, eigene Berechnungen

Welche Wirkungen hat die Benotung auf die Erfolgswahrscheinlichkeit einer Projekteinreichung? Diese Frage kann empirisch durch eine logistische Regression beantwortet werden (vgl. Hosmer, Lemeshow 1989). Es ist dies eine Regression, bei der die abhängige Variable binär $(0,1)$ ist und daher als Wahrscheinlichkeit interpretiert werden kann. Die zu schätzende Gleichung lautet:

(1) $\operatorname{prob}(y=1)=\frac{e^{X \beta}}{1+e^{X \beta}}$

$\operatorname{prob}(y=1)$ bezeichnet die Wahrscheinlichkeit, dass ein Projekt abgelehnt wird. $X$ ist der Vektor der erklärenden Variablen, $\beta$ der Koeffizientenvektor. Die Ergebnisse der Schätzung sind in Tabelle 6 wiedergegeben. 
Tabelle 6: Ergebnisse logistische Regression

\begin{tabular}{lrrrr}
\hline Variable & Koeffizient & Wald & Signifikanz & odds ratio \\
\hline Konstante & $\mathbf{1 0 , 1 8 3}$ & $\mathbf{2 0 , 1 5 3}$ & $\mathbf{, 0 0 0}$ & \\
Soziale Aspekte &,- 43 &, 31 &, 575 &, 64 \\
Externe Effekte &, 58 & 1,98 &, 158 & 1,78 \\
Verwertbarkeit &, 02 &, 01 &, 908 & 1,02 \\
Markterfahrung &,- 21 & 2,24 &, 134 &, 81 \\
Marktaussicht & $\mathbf{- , 4 6}$ & $\mathbf{7 , 7 3}$ & $\mathbf{, 0 0 5}$ & $\mathbf{, 6 3}$ \\
Management &, 04 &, 07 &, 781 & 1,03 \\
Leistungsfähigkeit &, 04 & 1,06 &, 302 & 1,05 \\
Durchführbarkeit &,- 12 & 1,02 &, 312 &, 88 \\
F\&E-Dynamik &,- 06 &, 10 &, 751 &, 94 \\
'Know How' Zuwachs & $\mathbf{- , 1 5}$ & $\mathbf{3 , 0 0}$ & $\mathbf{, 0 8 3}$ & $\mathbf{8 6}$ \\
Umwelt &, 12 &, 15 &, 701 & 1,12 \\
Nutzen & $\mathbf{- , 6 1}$ & $\mathbf{7 , 2 1}$ & $\mathbf{, 0 0 7}$ & $\mathbf{, 5 4}$ \\
Risiko &,- 08 &, 79 &, 371 &, 93 \\
Neuheit & $\mathbf{- , 2 1}$ & $\mathbf{3 , 4}$ & $\mathbf{, 0 6 5}$ & $\mathbf{8 1}$ \\
\hline
\end{tabular}

Pseudo $\mathrm{R}^{2}$ (Nagelkerke): 0,65. ?²: 132,8

Einen signifikanten Effekt auf die Erfolgswahrscheinlichkeit eines Projektes haben die fettgeduckten Unterkategorien 'Marktaussicht', 'Know How' und 'Nutzen'. Interessant ist ein Blick auf das sogenannte 'odds ratio'. Es gibt an, um wie viele Einheiten sich das Chancenverhältnis $[\mathrm{P}($ abgelehnt $) / \mathrm{P}$ (akzeptiert)] ändert, wenn sich die jeweilige Benotung um eine Einheit ändert. Beispiel: Liegt das Chancenverhältnis eines Projektes, abgelehnt oder akzeptiert zu werden, bei 1:1 und bekommt ein Projekt einen Punkt mehr bei der Unterkategorie 'Marktaussicht', so verändert sich das Chancenverhältnis auf $0,63: 1$, bei zwei zusätzlichen Punkten auf $(0,63)^{2}: 1$. Vor allem bei 'Nutzen' und 'Marktaussicht' reduzieren höhere Punkte die Wahrscheinlichkeit eines Scheiterns dramatisch. Die eben geäußerte Sorge, dass die fehlende finanzielle Leistungsfähigkeit eine unangemessene Hürde für kleine Firmen darstellt, zerstreut sich insofern, als dass die 'Leitungsfähigkeit' keinen statistisch signifikanten Zusammenhang auf die Erfolgswahrscheinlichkeit hat. Die signifikanten Kategorien 'Nutzen' und 'Marktaussicht' unterstreichen die stark anwendungsbezogene Stoßrichtung des Programms.

Wir haben uns weiterhin angesehen, ob die ex ante Bewertung des FFF ein guter Prädiktor der ex post Projektergebnisse ist. Hier gibt es viele Indizien dafür, dass die Bewertungen des FFF eine hohe Zielgenauigkeit haben. Beispielsweise ergibt sich ein signifikanter Zusammenhang zwischen der Beurteilung der Marktaussicht durch den FFF mit den tatsächlichen Umsätzen aus den Projektergebnissen, was ganz eindeutig für die Markterfahrung der FFF Mitarbeiter spricht. ${ }^{5}$

Abschließend haben wir untersucht, ob sich die Zahl der Kriterien reduzieren lässt, ohne dass relevante Information verloren geht. $\mathrm{Zu}$ diesem Zweck wurde eine Faktoranalyse durchgeführt, mit Hilfe derer die 14 Unterkriterien zu vier zusammengefasst werden (Tabelle 7).

5 Die kumulierten Umsätze aus Projektergebnissen, die bei Antragstellung 9 Punkte in der Rubrik Marktaussichten bekommen haben, betrugen im Schnitt 1,4 Mio. €. Demgegenüber stehen Umsätze von 5,4 Mio. $€$ bei Projekten mit einer Benotung von 14 Punkten. Die Unterschiede sind durch einen T-Test positiv auf Signifikanz geprüft worden. 
Tabelle 7: $\quad$ Ergebnisse der Faktoranalyse

\begin{tabular}{llr}
\hline & Kriterium & Faktorladung \\
\hline Faktor 1- & Externe Effekte & 0,74 \\
Markterschließung, & Soziale Aspekte & 0,74 \\
Nutzenstiftung & Nutzen & 0,72 \\
& Markterfahrung & 0,70 \\
& Verwertung & 0,68 \\
& Durchführbarkeit & 0,57 \\
\hline Faktor 2- & Risiko & 0,79 \\
inhärente innovative & Neuheit & 0,77 \\
Gehalt & Know How Zuwachs & 0,76 \\
\hline Faktor 3- & Umwelt & 0,90 \\
??? & Marktaussichten & 0,80 \\
& F\&E-Dynamik & 0,75 \\
\hline Faktor 4 & Management & 0,81 \\
Leistungsfähigkeit & Finanzielle Leistungsfähigkeit & 0,75 \\
\hline Methode: Varimax Rotation, Kaiser Kriterium, 69\% der Varianz werden erklärt
\end{tabular}

Die Interpretation der Ergebnisse ist etwas schwierig, da es auf den ersten Blick keine klaren Gemeinsamkeiten der einzelnen in einem Faktor zusammenge schlos senen Kriterien gibt. Dies bedeutet auch, dass die vier Unterkategorien des FFF (technische Bewertung Firma und Projekt, sowie wirtschaftlich Bewertung Firma und Markt) nur bei der wirtschaftlichen Bewertung der Firma (Management und finanzielle Leistungsfähigkeit) eine direkte Entsprechung in der Faktoranalyse finden (Faktor 4). Faktor 1 beschreibt hier grob die äußeren Gegebenheiten und Faktor 2 den inhärenten innovativen Gehalt des Projekts. Faktor 3 ist zu inhomogen und kann nicht sinnvoll zu einem Oberbegriff zusammengefasst werden. 


\section{Charakterisierung der Förderwerber und der Kontroll- gruppe}

Im folgenden Kapitel werden die Teilnehmer und die Kontrollgruppe anhand der im Fragebogen abgefragten Kriterien miteinander verglichen. Es zeigen sich in vielerlei Hinsicht Unterschiede. Insgesamt sind die teilnehmenden Firmen größer, internationaler und innovationsfreudiger als die Firmen aus der Kontrollgruppe, wobei sich dieser Sachverhalt in einem gewissen Maße natürlich auch aus den Förderkriterien des FFF ergibt. Hier haben wir gesehen, die finanzielle Leistungsfähigkeit ein wichtiges Kriterien für die Förderentscheidung ist. Dementsprechend konnte beobachtet werden, dass bei den Teilnehmern nur 5\% der Firmen mittlerweile Konkurs anmelden mussten. Bei den abgelehnten Bewerben hingegen ist der Anteil mit $11,4 \%$ mehr als doppelt so hoch.

\subsection{Unternehmensgröße}

Die Unternehmensgröße ist im Rahmen eines Forschungsförderungsprogramms insofern ein wichtiger Parameter, als in der wirtschaftswissenschaftlichen Diskussion ein Zusammenhang zwischen Größe und Innovationsverhalten postuliert wird, die in einer Vielzahl von Studien mit unterschiedlichem Ergebnis getestet wurde (vgl. Cohen 1995 für einen Literaturüberblick). Die Diskussion geht auf Schumpeter (1942) zurück, der behauptete, dass innovativer Output überproportional zur Firmengröße wächst: "We have got to accept is that large scale establishment has come to be the most powerful engine of progress" (Schumpeter 1942, S. 106). Intuitiv ist einleuchtend, dass der Wert von Prozessinnovationen, mit deren Hilfe die Herstellungskosten gesenkt werden können, mit zunehmender Marktgröße steigt. Hinzu kommen mögliche Skalenvorteile im Forschungsprozess, Kapitalmarktrestriktionen, die bei kleineren Firmen $\mathrm{zu}$ höheren Finanzierungskosten führen und mögliche Komplementaritäten zwischen F\&E- und andern Aktivitäten (z.B. Marketing), die bei großen Firmen besser entwickelt sind. Auf der anderen Seite haben große Unternehmen mit Bürokratisierung und Kontrollproblemen zu kämpfen. Nicht zuletzt dürften Mitarbeiter in Großunternehmen weniger motiviert sein, da die eigene Leistung den Unternehmenserfolg nicht sichtbar beeinflusst (Scherer, Ross 1990). Aufgrund dieser beiden gegenläufigen Effekte kann man in der Praxis eine Arbeitsteilung im Innovationsprozess beobachtet, bei dem radikale Innovationen meist von kleinen Firmen kommen und die größeren Konzerne lediglich die Marktentwicklung übernehmen (Mueller, Tilton 1969). Bezogen auf die Forschungsförderung bedeutet dieser Befund, dass Programme, bei denen inkrementelle Innovationen oder die industrielle Entwicklung im Vordergrund stehen, sich eher an größere Unternehmen richten sollte. Im Folgenden vergleichen wir die Unternehmensgröße der teilnehmenden Firmen mit der der Nicht-Teilnehmer.

Tabelle 8 zeigt die Ergebnisse: Teilnehmende Firmen wiesen einen durchschnittlichen Umsatz von 33,2 Mio. $€$ (2001) auf, fast viermal soviel wie bei der Kontrollgruppe mit 7 Mio. $€$. Die Gruppe der abgelehnten Bewerber bewegt sich bei hoher Volatilität irgendwo dazwischen. Aufgrund der geringen Stichprobengröße, sollte dieser Gruppe allerdings nicht allzu viel Aufmerksamkeit geschenkt werden, weshalb sie im folgenden nicht immer mit berücksichtigt wird. Der beobachtbare 'Knick' 
in der Zeitreihe ist darauf zurückzuführen, dass viele Respondenten lediglich die Zahlen für 2001 mitgeteilt haben und die Stichprobengröße für dieses Jahr daher größer ist als für vergangenen Jahre. Aus diesem Grund dürften die Angaben für das Jahr 2001 auch besonders verlässlich sein.

Tabelle 8: $\quad$ Umsatzentwicklung [1.000 € ]

\begin{tabular}{lrrrrrrr}
\hline & 1995 & 1996 & 1997 & 1998 & 1999 & 2000 & 2001 \\
\hline Teilnehmer & 18.631 & 16.813 & 19.541 & 24.868 & 34.304 & 39.388 & 33.274 \\
abgelehnte Bewerber & 6.320 & 7.740 & 11.220 & 5.244 & 12.116 & 20.697 & 1.080 \\
Kontrollgruppe & 5.429 & 5.984 & 6.939 & 6.708 & 7.455 & 6.186 & 7.036 \\
\hline
\end{tabular}

Quelle: FFF, Quelle: Fragebogenerhebung

Teilnehmende Firmen haben nicht nur einen absolut höheren Umsatz, sondern sind auch deutlich schneller gewachsen als die Nicht-Teilnehmer. Wird der Umsatz im Jahr 1995 auf 100 normiert, so zeigt sich in den 6 Jahren des Betrachtungszeitraumes ein Umsatzwachstum von $80 \%$ verglichen mit $29 \%$ bei unserer Kontrollgruppe und $22 \%$ bei der österreichischen Holzwirtschaft insgesamt. Auf das Jahr herunter gerechnet, ergibt sich ein jährliches nominales Wachstum von knapp über $10 \%$ bei den Förderteilnehmern gegenüber 4,5\% bei der Kontrollgruppe.

Die Teilnehmer sind nicht nur in den letzten sechs Jahren stärker gewachsen als die Kontrollgruppe, sondern auch über einen längeren Zeitraum. Wie aus Tabelle 9 hervorgeht, sind teilnehmende Firmen deutlich jünger als Unternehmen aus der Kontrollgruppe. Nimmt man den Median als relevanten Parameter, so beträgt der Altersabstand fast 40 Jahre. Kombiniert man das relativ junge Alter der Teilnehmern mit ihren vergleichsweise hohen Umsätzen, so zeigt sich klar die langfristig größere Dynamik.

Tabelle 9: Gründungsjahr

\begin{tabular}{|c|c|c|c|}
\hline & Förderteilnehmer & abgelehnte Bewerber & Kontrollgruppe \\
\hline Durchschnitt & 1957 & 1970 & 1937 \\
\hline Median & 1966 & 1975 & 1928 \\
\hline Maximum & 2001 & 1993 & 2000 \\
\hline Minimum & 1755 & 1926 & 1870 \\
\hline
\end{tabular}

Quelle: Fragebogenerhebung

Die Entwicklung bei den Mitarbeitern hat einen in der Form ähnlichen Verlauf wie die Entwicklung der Umsatzzahlen (Tabelle 10). Allerdings ist hier das Wachstum deutlich schwächer und im gleichen Zeitraum hat sich die Anzahl der Mitarbeiter bei den Teilnehmern lediglich um 36\% erhöht. Bei der Kontrollgruppe hingegen um $80 \%$. Die österreichische Holzindustrie insgesamt hat im selben Zeitraum sogar $12 \%$ der Mitarbeiter abgebaut. Die unterschiedlichen Wachstumsraten bei Umsatz und Mitarbeitern haben dazu geführt, dass die Teilnehmer ihren anfänglichen Rückstand in der Arbeitsproduktivität (Umsatz/Mitarbeiter) gegenüber der Kontrollgruppe aufgeholt haben und nunmehr deutlich vorne liegen. Bei der Interpretation der Daten ist $\mathrm{zu}$ allerdings $\mathrm{zu}$ berücksichtigen, dass die Zahl der Mitarbeiter nur ein grober Maßstab für den Produktionsfaktor Arbeit ist, da Veränderungen in der geleisteten Arbeitszeit oder in der beruflichen Qualifikation keine Berücksichtigung finden. Ein Wachstum in der Arbeitsproduktivität ergibt sich entweder aus einer höheren Kapitalausstattung eines Arbeitsplatzes oder aus Innovationen, die eine besseren Nutzung des gegebenen Kapitalstocks erlauben. Die Teilnahme an der Sonderaktion Holzforschung dürfte hierbei einen positiven Einfluss gehabt haben. 
Tabelle 10: Durchschnittliche Anzahl an Mitarbeitern und Umsatz pro Mitarbeiter [1.000 $€$ ]

\begin{tabular}{llrrrrrrr}
\hline & 1995 & 1996 & 1997 & 1998 & 1999 & 2000 & 2001 \\
\hline Teilnehmer & Mitarbeiter & 152 & 119 & 153 & 179 & 234 & 287 & 206 \\
& Umsatz/Mitarbeiter & 122 & 140 & 128 & 138 & 146 & 137 & 161 \\
\hline Abgelehnte & Mitarbeiter & 57 & 61 & 74 & 38 & 85 & 135 & 14 \\
Bewerber & Umsatz/Mitarbeiter & 110 & 126 & 151 & 135 & 142 & 153 & 77 \\
\hline Kontrollgruppe & Mitarbeiter & 35 & 39 & 40 & 42 & 46 & 43 & 63 \\
& Umsatz/Mitarbeiter & 154 & 152 & 172 & 159 & 161 & 142 & 111 \\
\hline
\end{tabular}

Quelle: Fragebogenerhebung

\subsection{Exportaktivitäten}

Teilnehmende Firmen sind nicht nur größer, sondern auch exportorientierter. Der Zusammenhang zwischen Größe und Exportquote klingt offensichtlich, ist theoretisch allerdings nicht leicht herzuleiten (Schott 2001). Tabelle 11 zeigt, dass die aggregierte Exportquote bei den Teilnehmern mit 49\% (2001) deutlich höher ist als bei den Firmen aus der Kontrollgruppe mit $26 \%$.

Tabelle 11: Exportquoten [\%]

\begin{tabular}{lrrrrrrr}
\hline & 1995 & 1996 & 1997 & 1998 & 1999 & 2000 & 2001 \\
\hline Teilnehmer & 44 & 47 & 50 & 50 & 52 & 51 & 49 \\
Kontrollgruppe & 33 & 37 & 40 & 31 & 29 & 27 & 26 \\
\hline
\end{tabular}

Quelle: Fragebogenerhebung

Allerdings scheint die hohe Exportquote von einigen Ausreißern dominiert zu werden. Im Fragebogen wurde nämlich auch die Frage nach der räumlichen Verteilung der Absatzmärkte gestellt (Tabelle 12), und hier ergeben sich nur kleinere Unterschiede. Insbesondere hat der Anteil des europäischen Exportmarktes in etwa die gleiche Größenordnung. ${ }^{6}$ Der auffälligste Unterschied ist die Zusammensetzung des österreichischen Marktes. Firmen aus der Kontrollgruppe bedienen im Wesentlichen den regionalen Markt $(<100 \mathrm{~km})$, während bei den Teilnehmern der nationale Markt (> $100 \mathrm{~km}$ ) dominiert. Außerdem spielen außereuropäische Absatzmärkte bei der Kontrollgruppe eine untergeordnete Rolle, während sie bei den Teilnehmern immerhin knapp 10\% ausmachen.

Tabelle 12: Räumliche Absatzverteilung [\%]

\begin{tabular}{lrrr}
\hline & Teilnehmer & abgelehnter Bewerber & Kontrollgruppe \\
\hline Regional $(<100 \mathrm{~km})$ & 24,9 & 66,8 & 36,8 \\
National $(>100 \mathrm{~km})$ & 31,5 & 26,2 & 25,2 \\
Europäisch & 35,9 & 7,0 & 36,4 \\
Außereuropäisch & 7,8 & 0,0 & 1,4 \\
\hline
\end{tabular}

Quelle: Fragebogenerhebung

Eine detaillierte Analyse der Exportaktivitäten auf Branchenniveau wird in Kapitel 7 durchgeführt. Dort wird außerdem versucht, Entwicklungen in der Außenhandelsstruktur mit den Förderungen der Sonderaktion Holzforschung in Beziehung zu setzen.

6 Methodisch ist der Unterschied in den Exportquoten dadurch zu erklären, dass es sich bei der Exportquote um eine aggregierte Größe handelt, d.h. es wurde die Summe der Exporte durch die Summe der Umsätze über alle Firmen gebildet. Bei der Frage nach der räumlichen Absatzverteilung hingegen wurde der Durchschnitt der jeweiligen Anteile errechnet, was nicht dasselbe ist. 


\subsection{Eigentumsverhältnisse}

Eine wichtige Frage, die in der forschungsförderungspolitischen Diskus sion eine Rolle spielt, ist die nach der 'Nationalität' der Unternehmen. Schließlich werden die strategischen Unternehmensentscheidungen in der Hauptverwaltung getroffen und dort sind meist auch jene Tätigkeiten angesiedelt, die eine hohe Wertschöpfung erwirtschaften. Vor allem in strategisch wichtigen Hochtechnologiebereichen besteht oftmals eine Abneigung, Unternehmen im ausländischen Eigentum zu unterstützen, da Forschungsergebnisse sofort ins Ausland transferiert werden. Dieser Sachverhalt kann in der Folge die Nachhaltigkeit eines Programms gefährden.

Dieses Problem stellt sich in der österreichischen Holzbranche jedoch nicht. Wie Tabelle 13 zeigt, ist die Holzindustrie insgesamt eine sehr 'österreichische' Industrie und nur wenige Betriebe - jeweils unter $4 \%$ - befinden sich in ausländischem Eigentum.

Tabelle 13: Unternehmensstatus [\%]

\begin{tabular}{lrrr}
\hline & Teilnehmer & $\begin{array}{r}\text { abgelehnte } \\
\text { Bewerber }\end{array}$ & Kontrollgruppe \\
\hline Unternehmensstammsitz & 90,9 & 100,0 & 88,9 \\
Zweigbetrieb im inländischen Mehrheitsbesitz & 3,9 & 0,0 & 3,7 \\
Zweigbetrieb im ausländischen Mehrheitsbesitz & 3,9 & 0,0 & 3,7 \\
keine Angabe & 1,3 & 0,0 & 3,7 \\
\hline
\end{tabular}

Quelle: Fragebogenerhebung

Eine interessante Frage ist außerdem, inwieweit Management und Eigentum getrennt sind. In Hinblick auf das Innovationsverhalten wird die Trennung zwischen Mana gement und Eigentum in der Literatur eher skeptisch beurteilt und bereits Schumpeter hat die zentrale Bedeutung des 'Entrepreneurs' für die wirtschaftliche Entwicklung herausgestrichen. In Bezug auf risikoreiche F\&E-Vorhaben wird ein angestellter Manager eher risikoscheu sein, da er bei einem Scheitern möglicherweise bestraft wird, aber im Falle eines Erfolges nur teilweise von den Erträgen profitiert. Theoretische Modelle postulieren daher, dass angestellte Manager eher weniger in innovative Tätigkeiten investieren (Aghion, Dewatripont, Rey 1997). Empirische Untersuchungen bestätigen diese Theorie (Kraft 1989).

Tabelle 14: $\quad$ Trennung von Eigentum und Management [\%]

\begin{tabular}{lrrr}
\hline & Teilnehmer & $\begin{array}{r}\text { abgelehnte } \\
\text { Bewerber }\end{array}$ & $\begin{array}{r}\text { Kontroll- } \\
\text { gruppe }\end{array}$ \\
\hline Geschäftsführer ist nicht Eigentümer & 33,8 & 0,0 & 0,0 \\
Geschäftsführer ist Alleineigentümer & 31,2 & 40,0 & 55,6 \\
Geschäftsführer ist Mehrheitseigentümer $(>50 \%)$ & 16,9 & 20,0 & 25,9 \\
Geschäftsführer ist Minderheitseigentümer $(<50 \%)$ & 16,9 & 40,0 & 14,8 \\
keine Angaben & 1,3 & 0,0 & 3,7 \\
\hline
\end{tabular}

Quelle: Fragebogenerhebung

Tabelle 14 gibt die Ergebnisse der Fragebogenerhebung wieder. Bei immerhin einem Drittel der Teilnehmer ist gibt es eine Trennung zwischen Eigentum und Management. Bei der Kontrollgruppe hingegen ist der Geschäftsführer in der Regel auch gleichzeitig Eigentümer. Offenbar dominiert bei der Kontrollgruppe der Typ des Familienunternehmens, das entweder selber aufgebaut oder ererbt wurde. Bei den Teilnehmern gibt es demgegenüber wesentlich häufiger ein professionelles Management, das von den Eigentümern eingesetzt wurde. Insgesamt korreliert allerdings die Firmengröße mit der Neigung zu einem externen Management. Der 
eben gemachte Befund gilt aber auch dann, wenn man nur gleichgroße Firmen miteinander vergleicht.

\subsection{F\&E-Verhalten}

Die für die Evaluierung eines Forschungsförderungsprogramms wesentlichen Parameter sind die innovationsbezogenen, wobei man hier Input- und Outputindikatoren unterscheiden kann. Inputs im Innovationsprozess sind die Forschungsausgaben und auf der Outputseite sind es neue Produkte und Produktionsprozesse. Eine wichtige Grundsatzfrage, die Auskunft über den Stellenwert von Innovation in einer Firma liefert, ist die nach der Existenz eines eigenen F\&E-Budgets bzw. einer eigenen F\&E-Abteilung. Hier zeigt sich, ob Innovation innerhalb einer Firma nachhaltig verankert ist, da es hier um Tatbestände geht, die eine gewisse Struktur haben und nicht schnell rückgängig zu machen sind.

Tabelle 15: F\&E- bzw. Innovationsinfrastruktur [\%]

\begin{tabular}{lrrrr}
\hline & Förderteilnehmer & abgelehnte Bewerber & Kontrollgruppe \\
\hline eigenes F\&E-Budget & 64 & 20 & 23 \\
eigene F\&E-Abteilung & 47 & 0 & 4 \\
\hline Quelle: Fragebogenerhebung & & &
\end{tabular}

Quelle: Fragebogenerhebung

Nur 23\% der Firmen aus der Kontrollgruppe verfügt überhaupt über ein eigenes F\&E-Budget (Tabelle 15). Dementsprechend niedrig ist mit 0,3\% die Forschungsquote. Die Teilnehmer unterscheiden sich in diesem Punkt deutlich. Immerhin fast zwei Drittel haben ein eigenes F\&E-Budget, und im Schnitt wurden 1,6\% (2001) des Umsatzes für F\&E ausgeben (Tabelle 16). Im Vergleich mit anderen Branchen ist allerdings auch diese Quote eher gering. ${ }^{7}$ Das Maximum erreichte die F\&E-Quote 1999, seitdem ist ein abnehmender Trend $\mathrm{zu}$ erkennen. Inwieweit hier eine Kausalität mit dem Auslaufen des Förderprogramms besteht, wird in Kapitel 5 unter der Überschrift 'Additionalitäten' behandelt.

Tabelle 16: Entwicklung der F\&E-Quote [\%]

\begin{tabular}{lrrrrrrr}
\hline & 1995 & 1996 & 1997 & 1998 & 1999 & 2000 & 2001 \\
\hline Teilnehmer & 1,61 & 1,73 & 1,95 & 2,02 & 2,03 & 1,87 & 1,62 \\
Kontrollgruppe & 0,1 & 0,1 & 0,1 & 0,1 & 0,1 & 0,7 & 0,3 \\
\hline
\end{tabular}

Quelle: FFF, Fragebogenerhebung

Den Großteil der Forschungsausgaben stellen in der Regel die Personalkosten dar. Dementsprechend beschäftigen die 'forschungsintensiven' Förderteilnehmer deutlich mehr hochqualifiziertes Personal (Tabelle 17). 80\% der Teilnehmer beschäftigen mindestens einen Akademiker, bei der Kontrollgruppe hingegen ist dies nur rund die Hälfte. Der höhere Anteil von Maturanten und Akademiker geht auf Kosten des Facharbeiteranteils, der bei der Kontrollgruppe rund 10 Prozentpunkte höher ist.

7 Firmen aus forschungsintensiven Branchen wie der Pharmaindustrie geben bis zu $20 \%$ des Umsatzes für F\&E aus. 
Tabelle 17: Bildungsabschluss der Mitarbeiter [\%]

\begin{tabular}{lrrr}
\hline & Teilnehmer & abgelehnte Bewerber & Kontrollgruppe \\
\hline Akademiker & 10,9 & 4,6 & 1,9 \\
HTL/HAK Absolventen & 11,0 & 12,1 & 5,8 \\
Maturanten & 4,5 & 0,8 & 1,1 \\
Facharbeiter & 41,9 & 73,5 & 52,6 \\
Ungelernte & 30,0 & 8,5 & 37,9 \\
Leihpersonal & 1,3 & 0,4 & 0,6 \\
\hline
\end{tabular}

Quelle: Fragebogenerhebung

F\&E-Ausgaben bzw. das Forschungspersonal stellen lediglich einen Inputfaktor im F\&E-Prozess dar, auf der Outputseite sind Patente ein beliebter Indikator für Inventionen, also neuen Produkten oder Prozessen. ${ }^{8}$ Auch hier liegen die Teilnehmer mit durchschnittlich fünf Patenten auf 1.000 Mitarbeiter und Jahr klar vorne (Tabelle 18). Bei Unternehmen aus der Kontrollgruppe sind überhaupt nur in einem Jahr Patente angefallen. Die Varianz sowohl innerhalb der Gruppen als auch im Zeitverlauf ist hier allerdings extrem hoch.

Tabelle 18: Entwicklung der Patentanmeldungen [je 1.000 Mitarbeiter]

\begin{tabular}{lrrrrrrr}
\hline & 1995 & 1996 & 1997 & 1998 & 1999 & 2000 & 2001 \\
\hline Teilnehmer & 8,5 & 8,8 & 6,2 & 2,9 & 3,7 & 3,6 & 5,2 \\
Kontrollgruppe & 0 & 0 & 0 & 0 & 0 & 3,8 & 0 \\
\hline
\end{tabular}

Quelle: Fragebogenerhebung

Um den Zusammenhang zwischen Patenanmeldungen und Forschungsausgaben zu überprüfen, haben wir den Patenfunktion als Poissonverteilten Zufallsprozess modelliert, der folgende Form hat:

(2) $\operatorname{Prob}(y)=e^{-?} ?^{y} / y !$

Die Wahrscheinlichkeit einer Entdeckung, die zu y Patenten führt, ist zwar ein zufälliges Ereignis, dem Zufall kann allerdings mit zwei Einflussgrößen auf die Sprünge geholfen werden; den Forschungsausgaben und der Unternehmensgröße (Schumpeter-Hypothese). Geschätzt wird der Parameter $\lambda$ aus Gleichung (2):

(3) $\lambda_{i t}=\exp \left(X_{i i} \beta\right)$

$\mathrm{X}$ ist der Vektor der erklärenden Variablen, in unserem Fall die F\&E-Ausgaben und der Umsatz (jeweils in Mio. €). Die Daten sind in Panelform und der Index $i$ kennzeichnet die Firmen und $t$ das Jahr. Da die Annahmen des Poisson-Modells sehr restriktiv $\operatorname{sind}^{9}$, wird alternativ ein Fehlerterm $e$ eingeführt, der zur sogenannten negativen Binomialverteilung führt:

(4) $\lambda_{i t}=\exp \left(X_{i \uparrow} \beta+e_{i}\right)$

Die in Tabelle 19 dargestellten Ergebnisse legen zweierlei Schlüsse nahe: Einerseits ist die Patentneigung in der gesamten Holzbranche im allgemeinen sehr schwach und auf $€ 1,1$ Mio. Forschungsausgaben entfällt lediglich ein Patent. Entweder werden

8 In diesem Zusammenhang unterscheidet man zwischen der Erfindung (Invention) und der Markteinführung eines neuen Produktes oder Prozesses (Innovation).

9 Es wird angenommen, dass der Mittelwert gleich der Varianz ist. 
nur wenig schützenswerte Erfindungen gemacht oder die Firmen nutzen andere Mechanismen, ihre Innovationen vor Nachahmung zu schützen (z.B. 'first mover advantage'). Wenn Innovationen eher auf firmenspezifischen Routinen basieren, sind sie schwer zu kopieren und ein Patentschutz nicht zwingend erforderlich.

Tabelle 19: Ergebnis Patentschätzung

\begin{tabular}{l|rrr|rrr}
\hline & \multicolumn{3}{|c|}{ Poisson (3) } & \multicolumn{3}{c}{ Neg. Binomial (4) } \\
\cline { 2 - 7 } & Koeffizient & $\mathrm{z}$ & Signifikanz & Koeffizient & $\mathrm{z}$ & Signifikanz \\
\hline F\&E & .8518 & 4.05 & 0.000 & .9262 & 4.79 & 0.000 \\
Umsatz & -0.0095 & -3.57 & 0.000 & -.0103 & -3.99 & 0.000 \\
\hline
\end{tabular}

Geschätzt wurden jeweils 'random effects' Modelle

Quelle: Fragebogenerhebung, eigene Berechnungen

Außerdem sieht man anhand des negativen Vorzeichens der Umsatzvariablen, dass ein gegebener $€$ an Forschungsausgaben in einem kleineren Unternehmen eher zu einem Patent führt als in einem größerem Unternehmen, was die Wichtigkeit unterstreicht, sich als Förderinstitution um kleinere Unternehmen zu kümmern. Bereits in der Zwischenevaluierung wurde hervorgehoben, dass kleinere Unternehmen oftmals die innovativeren sind (Ohler, Teischinger, Schörghofer 1998).

Aufgrund der eher schlechten Datenqualität bei dieser Frage ${ }^{10}$, sollten aus dieser Analyse allerdings keine zu ambitionierten Schlüsse gezogen werden. Nicht zuletzt sind Patente nur ein Zwischenschritt im Innovationsprozess und nur eine geringer Teil von angemeldeten Patenten hat einen positiven ökonomischen Wert (Harhoff, Narin, Scherer, Vopel 1999).

\subsection{Innovationsbarrieren}

Die hohe Unsicherheit bei Patentanmeldungen unterstreicht wieder einmal, dass Innovation ein risikoreiches Unterfangen ist. Dieser Sachverhalt wird auch von allen Firmen so gesehen und auf die Frage nach den wichtigsten Innovationsbarrieren wurden von allen Firmen genannt: (i) Innovationen sind mit $\mathrm{zu}$ hohem Risiko verbunden, (ii) die Finanzierung von Innovationen ist zu schwierig, (iii) die eigenen Forschungskapazitäten sind zu klein (Tabelle 20). Diese drei Punkte sind im Wesentlichen auch die einzigen, deren Wichtigkeit jenseits der Mittelwertes von 3 angesiedelt sind. Alle anderen zur Auswahl stehenden Antworten scheinen nicht wirklich allzu große Probleme zu verursachen.

10 Viele Unternehmen haben die Frage nach Patenten nicht beantwortet und deswegen basiert die Schätzung auf lediglich 90 Beobachtungen. 
Tabelle 20: Innovationsbarrieren

\begin{tabular}{|c|c|c|c|c|c|c|}
\hline & \multicolumn{2}{|c|}{ Teilnehmer } & \multicolumn{2}{|c|}{$\begin{array}{l}\text { abgelehnte } \\
\text { Bewerber }\end{array}$} & \multicolumn{2}{|c|}{$\begin{array}{l}\text { Kontroll- } \\
\text { gruppe }\end{array}$} \\
\hline & Rang & $\varnothing$ & Rang & $\varnothing$ & Rang & $\varnothing$ \\
\hline zu hohe Innovationskosten bzw. Risiken & 1 & 3,45 & 1 & 4,20 & 3 & 3,27 \\
\hline fehlende Innovationsfinanzierung & 2 & 3,30 & 4 & 3,00 & 2 & 3,30 \\
\hline zu geringe eigene $\mathrm{F} \& \mathrm{E}-\mathrm{Kapazitäten}$ & 3 & 3,26 & 3 & 3,20 & 1 & 3,41 \\
\hline zu hohe technische Risiken & 4 & 2,88 & 2 & 3,60 & 5 & 3,09 \\
\hline Mangel an qualifiziertem Personal & 5 & 2,79 & 7 & 2,60 & 9 & 2,50 \\
\hline mangelende externe Unterstützung & 6 & 2,53 & 6 & 2,80 & 7 & 2,90 \\
\hline $\begin{array}{l}\text { Mangel an Management- und } \\
\text { Organisations-Know-How }\end{array}$ & 7 & 2,34 & 9 & 2,50 & 11 & 2,45 \\
\hline $\begin{array}{l}\text { bisher keine ernsthaften Probleme } \\
\text { aufgetreten }\end{array}$ & 8 & 2,34 & 12 & 2,00 & 10 & 2,50 \\
\hline fehlende Kooperationsmöglichkeiten & 9 & 2,29 & 5 & 3,00 & 6 & 3,00 \\
\hline fehlende technologische Möglichkeiten & 10 & 2,25 & 8 & 2,60 & 8 & 2,73 \\
\hline Gesetze, Regulierungen & 11 & 2,18 & 11 & 2,20 & 4 & 3,10 \\
\hline $\begin{array}{l}\text { fehlende Nachfrage nach innovativen } \\
\text { Produkten }\end{array}$ & 12 & 2,14 & 10 & 2,40 & 13 & 2,26 \\
\hline $\begin{array}{l}\text { kein aktueller Bedarf aufgrund bereits } \\
\text { erfolgter Innovationen }\end{array}$ & 13 & 2,08 & 13 & 1,80 & 14 & 1,91 \\
\hline $\begin{array}{l}\text { Widerstand gegenüber Veränderungen } \\
\text { innerhalb des Unternehmens }\end{array}$ & 14 & 2,08 & 14 & 1,60 & 12 & 2,32 \\
\hline
\end{tabular}

Skala: 1: 'trifft gar nicht zu', 5: 'trifft sehr zu'

Quelle: Fragebogenerhebung

Die größten Unterschiede im Antwortverhalten zwischen den Teilnehmern und der Kontrollgruppe sind beim Punkt 'Gesetze und Regulierungen' zu beobachten. Für die Kontrollgruppe stellen Gesetze und Regulierungen eine wesentlich höhere Hürde dar als für die deutlich innovativere Gruppe der Teilnehmer. Es ist nicht auszuschließen, dass sich hier in erster Linie Vorurteile manifestieren. Ein weiterer Unterschied ist bei dem Punkt 'fehlende Kooperationspartner' zu erkennen. Für die Teilnehmer stellt dies eine wesentlich geringere Innovationsbarriere dar als für die Firmen der Kontrollgruppe. Auch wenn es schwer ist, hier Kausalitäten herzustellen, kann vermutet werden, dass der FFF bei seinem Bemühen, Kooperationsprojekte zu fördern, erfolgreich war. Kooperationen und Zusammenarbeit zwischen Firmen und Forschungseinrichtungen zu fördern war schließlich ein explizites Ziel der Sonderaktion Holzforschung. Das dieses Ziel erreicht zu sein scheint, zeigt auch Tabelle 21, aus der deutliche Unterschiede bei den Kooperationspartnern sichtbar werden.

Tabelle 21: Kooperationspartner im Bereich Holzforschung [\%]

\begin{tabular}{lrr}
\hline & Teilnehmer & Kontrollgruppe \\
\hline F\&E-Einrichtungen in Österreich & 60,9 & 22,7 \\
Universitäten in Österreich & 41,3 & 9,1 \\
KMU in Österreich & 36,5 & 4,6 \\
F\&E-Einrichtungen im Ausland & 24,1 & 5,3 \\
Großunternehmen in Österreich & 21,7 & 10,5 \\
Großunternehmen im Ausland & 20,7 & 10 \\
Universitäten im Ausland & 20,7 & 0 \\
KMU im Ausland & 10,9 & 5 \\
\hline
\end{tabular}

(Mehrfachnennungen)

Quelle: Fragebogenerhebung

Man erkennt deutlich, dass die teilnehmenden Firmen besser vernetzt sind als die Unternehmen der Kontrollgruppe. Der Unterschied bleibt auch bestehen, wenn man 
nur diejenigen Firmen berücksichtigt, die ein eigenes F\&E-Budget haben, mithin also forschungsaffin sind. Insgesamt zeigt sich auch, dass die inländischen außeruniversitären Forschungsinstitute die häufigsten Kooperationspartner sind. Zu Partnern im Ausland gibt es hingegen eher seltene Kontakte. Offenbar gibt es eine größere Schwellenangst, internationale Partnerschaften und Kooperationen einzugehen. Die Förderung internationaler Kooperationen war bereits jetzt ein Programmziel, sollte aber in folgenden Programmen noch weiter ausgebaut werden.

\subsection{Marktpositionierung}

Der folgende Abschnitt zeigt die unterschiedliche Marktpositionierung von Teilnehmern und Kontrollgruppe auf. Gemeinsam mit dem FFF haben wir die Produktpalette 'Endprodukte', 'Halbfertigprodukte' und 'Grundstoffe' zugeordnet. Es zeigt sich, dass Teilnehmer eher Produkte an bieten, die weiter 'downstream' in der Wertschöpfungskette stehen (Tabelle 22).

\section{Tabelle 22: Kategorisierung anhand des wichtigsten Produkts [\%]}

\begin{tabular}{lrr} 
& Teilnehmer & Kontrollgruppe \\
\hline Endprodukte & 57,1 & 19,1 \\
Halbfertigprodukte & 20 & 30,8 \\
Grundstoffe & 5,7 & 46,1 \\
nicht zuordenbar & 17,1 & 3,9 \\
\hline
\end{tabular}

Quelle: Fragebogenerhebung

In der Holzbranche sind dies marketingintensive Produkte mit verhältnismäßig hoher Wertschöpfung. Obwohl wir keine Daten über die von den Firmen erwirtschaftete Wertschöpfung - also der Summe aus Löhnen, Gehältern und Gewinnen - haben, ist zu vermuten, dass die teilnehmenden Firmen hier deutlich vorne liegen. Nicht zuletzt haben die Teilnehmer weniger Wettbewerber als die Kontrollgruppe (6,5 gegenüber 25). Dies legt die Vermutung nahe, dass sie es eher geschafft haben, ihre Produkte zu differenzieren und in der Lage sind, am Markt höhere Preise durchzusetzen. Allerdings empfindet die Mehrheit der Firmen, dass der Preisdruck in den letzten Jahren härter geworden ist und nur über erstklassige Produktqualität geführt werden kann (Tabelle 23 und Tabelle 24).

\section{Tabelle 23: Entwicklung des Preisdrucks [\%]}

\begin{tabular}{lrr}
\hline & Teilnehmer & Kontrollgruppe \\
\hline Preisdruck hat zugenommen & 61,04 & 55,56 \\
Preisdruck hat eher zugenommen & 35,06 & 25,93 \\
neutral & 1,30 & 11,11 \\
Preisdruck hat eher abgenommen & 1,30 & 3,70 \\
keine Angaben & 1,30 & 3,70 \\
\hline
\end{tabular}

Quelle: Fragebogenerhebung

Aus Tabelle 24 wird deutlich, dass Produktinnovationen bei den Förderteilnehmern als Wettbewerbsvorteil an zweiter Stelle nach der Produktqualität und vor der Qualität des Personals rangieren. Eine untergeordnete Stelle spielen bei beiden Gruppen der Standort, die räumliche Nähe zu den Kunden und interessanterweise die Finanzkraft. Dies ist insofern etwas erstaunlich, als bei den Innovationsbarrieren an erster Stelle die hohen Kosten genannt wurden. Daher hätte man erwartet, dass der finanziellen Leistungsfähigkeit hier eine größere Bedeutung zukommt. 
Tabelle 24. Eigene Stärken und Wichtigkeit für Wettbewerbsfähigkeit

\begin{tabular}{|c|c|c|c|c|c|c|c|c|c|c|}
\hline & \multicolumn{4}{|c|}{ eigene Stärken (S) } & \multicolumn{4}{|c|}{$\begin{array}{c}\text { Wichtigkeit für } \\
\text { Wettbewerbsfähigkeit }(\mathrm{W})\end{array}$} & \multicolumn{2}{|c|}{ Differenz $(\mathrm{W}-\mathrm{S})$} \\
\hline & \multicolumn{2}{|c|}{ Teilnehmer } & \multicolumn{2}{|c|}{$\begin{array}{c}\text { Kontroll- } \\
\text { gruppe }\end{array}$} & \multicolumn{2}{|c|}{ Teilnehmer } & \multicolumn{2}{|c|}{$\begin{array}{c}\text { Kontroll- } \\
\text { gruppe }\end{array}$} & $\begin{array}{c}\text { Teil- } \\
\text { nehmer }\end{array}$ & $\begin{array}{c}\text { Kontroll } \\
\text { gruppe }\end{array}$ \\
\hline & Rang & $\varnothing$ & Rang & $\varnothing$ & Rang & $\varnothing$ & Rang & $\varnothing$ & & \\
\hline Einkauf & 11 & 2,31 & 3 & 1,74 & 13 & 2,04 & 3 & 1,32 & $-0,26$ & $-0,42$ \\
\hline Finanzkraft & 15 & 2,56 & 14 & 2,52 & 15 & 2,17 & 13 & 2,00 & $-0,39$ & $-0,52$ \\
\hline Flexibilität & 2 & 1,67 & 2 & 1,67 & 5 & 1,60 & 2 & 1,32 & $-0,07$ & $-0,35$ \\
\hline Image/Markenname & 6 & 2,01 & 7 & 2,13 & 10 & 1,93 & 12 & 2,00 & $-0,08$ & $-0,13$ \\
\hline Innovationstätigkeit & 3 & 1,91 & 16 & 2,65 & 2 & 1,52 & 9 & 1,81 & $-0,38$ & $-0,84$ \\
\hline $\begin{array}{l}\text { Kommunikation, } \\
\text { Kooperation }\end{array}$ & 8 & 2,11 & 10 & 2,35 & 12 & 2,03 & 15 & 2,10 & $-0,08$ & $-0,25$ \\
\hline Kundennähe & 10 & 2,15 & 9 & 2,27 & 14 & 2,11 & 11 & 1,90 & $-0,04$ & $-0,37$ \\
\hline Logistik & 13 & 2,48 & 11 & 2,35 & 11 & 2,01 & 14 & 2,09 & $-0,46$ & $-0,26$ \\
\hline Marketing/Vertrieb & 12 & 2,40 & 8 & 2,21 & 4 & 1,58 & 6 & 1,50 & $-0,82$ & $-0,71$ \\
\hline Personal/Qualifikation & 4 & 1,96 & 5 & 1,91 & 3 & 1,55 & 4 & 1,38 & $-0,41$ & $-0,53$ \\
\hline Preis & 16 & 2,59 & 15 & 2,52 & 8 & 1,82 & 10 & 1,90 & $-0,77$ & $-0,62$ \\
\hline Produktionstechnik & 9 & 2,12 & 13 & 2,48 & 9 & 1,91 & 8 & 1,76 & $-0,21$ & $-0,72$ \\
\hline Produktqualität & 1 & 1,66 & 1 & 1,48 & 1 & 1,40 & 1 & 1,27 & $-0,26$ & $-0,21$ \\
\hline Qualitätswesen & 7 & 2,04 & 4 & 1,79 & 6 & 1,60 & 5 & 1,50 & $-0,44$ & $-0,29$ \\
\hline Service/Servicequalität & 5 & 2,01 & 6 & 2,05 & 7 & 1,68 & 7 & 1,57 & $-0,33$ & $-0,48$ \\
\hline Standort & 14 & 2,48 & 12 & 2,43 & 16 & 2,82 & 16 & 2,76 & 0,34 & 0,33 \\
\hline
\end{tabular}

Skala: 1: 'sehr wichtig', 5: 'sehr unwichtig'

Quelle: Fragebogenerhebung

Auffallend ist, dass die Firmen ihre Stärken durchgehend schlechter einschätzen als die jeweilige Wichtigkeit für die Wettbewerbsfähigkeit (Spalte 3: Differenz). Offenbar glauben beide Gruppen, Nachholbedarf bei den für die Wettbewerbsfähigkeit entscheidenden Kompetenzen zu haben. Dies zeigt, dass die meisten Unternehmen ihrer eigenen Einschätzung nach noch nicht gut genug für den Wettbewerb gerüstet sind. Besonders offensichtlich ist dieser Befund für die Teilnehmer bei den Kategorien 'Preis', 'Marketing' und 'Logistik'. Die Firmen scheinen sich so einzuschätzen, dass sie zwar das Geschäft von der Produktseite verstehen, es aber noch Verbesserungspotenzial bei den vertriebsrelevanten Punkten gibt. Bei der Kontrollgruppe klafft die größte Lücke bei dem Punkt 'Innovationstätigkeit', dessen Wichtigkeit für die Wettbewerbsfähigkeit zwar gesehen wird, bei dem die eigenen Fähigkeiten allerdings am schlechtesten eingeschätzt werden. Die Sensibilisierung für das Thema Innovation scheint also auch bei Firmen aus der Kontrollgruppe vorzuliegen. Dies ist umso wichtiger, als dass auch hier die Firmen nicht über einen Preiswettbewerb konkurrieren können und der Preis bei den eigenen Stärken an vorletzter Stelle genannt wird. Hohe Preise sind aber nur über innovative Produkte zu erzielen, die dem Kunden einen hohen Nutzen liefern und für die es dementsprechend eine hohe Zahlungsbereitschaft gibt. Das derzeitige Stärkenprofil, bei dem die beiden Punkte 'Preis' und 'Innovationstätigkeit' zusammen an letzter Stelle rangieren, ist bedenklich und kann sicher nicht auf Dauer aufrecht erhalten werden. 


\section{Die Einschätzung des Förderprogramms}

Nachdem im letzten Abschnitt die teilnehmenden Firmen auf einer allgemeinen Ebene charakterisiert und dabei der Kontrollgruppe gegenübergestellt wurden, werden in diesem Kapitel die konkreten Erfahrungen mit der Sonderaktion Holzforschung in den Mittelpunkt der Betrachtung gerückt. Der gesamte Projektzyklus von der Kontaktaufnahme über die Förderabwicklung wird dabei von den befragten Förderwerbern benotet. Außerdem werden die mit der Sonderaktion Holzforschung verbundenen Ziele und der Stellenwert des Programms in der Unternehmensstrategie diskutiert. Für den FFF lassen sich daraus Schlüsse ziehen, inwiefern die von ihm gesetzten Programmziele auch tatsächlich erreicht wurden.

\subsection{Bekanntheit des Programms und Kontaktaufnahme}

Einleitend steht die Frage, durch wen die Unternehmen auf die Sonderaktion Holzforschung aufmerksam geworden sind. Wie Tabelle 25 zeigt, ist der mit Abstand größte Teil der Teilnehmer direkt über den FFF zur Sonderaktion gekommen. Dies spricht dafür, dass der FFF in der Branche präsent ist und über eine hohe Reichweite verfügt.

Tabelle 25: Wie wurden die Förderteilnehmer auf die Sonderaktion Holzforschung aufmerksam? [\%]

\begin{tabular}{lrr}
\hline & Teilnehmer & abgelehnter Bewerber \\
\hline Lieferanten & 0,0 & 20,0 \\
Kunden & 1,4 & 20,0 \\
Internet & 2,7 & 0,0 \\
WIFI & 2,7 & 20,0 \\
ProHolz & 4,1 & 0,0 \\
Holzcluster & 4,1 & 0,0 \\
Universität/Forschungseinrichtung & 8,1 & 0,0 \\
Wirtschaftskammer/Fachverband & 9,5 & 20,0 \\
Informationsveranstaltung & 9,5 & 20,0 \\
Berater & 10,8 & 40,0 \\
Bank & 11,0 & 20,0 \\
FFF & 74,3 & 20,0 \\
\hline Quelle: Fragebogenerhebung
\end{tabular}

Quelle: Fragebogenerhebung

Auf der anderen Seite hat allerdings immerhin 30\% der Kontrollgruppe angegeben, dass sie von dem Programm nichts gewusst haben und deswegen nicht daran teilgenommen haben (Tabelle 26). Es ist allerdings die Frage, inwieweit hier eine Holschuld der Industrie bzw. eine Bringschuld des FFF vorliegt. Da wir gesehen haben, dass die meisten Unternehmen aus der Kontrollgruppe eher sporadisch innovieren, kann davon ausgegangen werden, dass die relevante Zielgruppe auch erreicht wurde.

Erstaunlich ist die Tatsache, dass sich 2/3 der Unternehmen aus der Kontrollgruppe vorstellen können, in Zukunft an einer Neuauflage der Sonderaktion Holzforschung teilzunehmen. Nur 29,6\% schließen eine Teilnahme dezidiert aus, obwohl die meisten Firmen dieser Gruppe bisher keine relevanten Forschungsprojekte unternommen hat. Realistischerweise dürfte es daher in der Kontrollgruppe nicht mehr als eine Handvoll wirklich ernsthafter Interessenten geben. Sollten diese bei einer tatsäch- 
lichen Neuauflage der Sonderaktion neu dazu kommen, wäre das trotzdem ein Erfolg, der durch diese Fragebogenaktion ausgelöst worden wäre.

\section{Tabelle 26: Gründe für Nichtteilnahme [\%]}

\begin{tabular}{lr}
\hline machen keine Projekte, die für eine Förderung in Frage kommen & 50,0 \\
Programm war nicht bekannt & 31,8 \\
wir nehmen an anderen Förderaktionen teil & 22,7 \\
Kosten und Aufwand einer Antragstellung sind zu hoch & 13,6 \\
nehmen grundsätzlich keine staatliche Förderungen in Anspruch & 9,1 \\
schlechte Erfahrungen mit Förderprogrammen & 4,5 \\
\hline (Mehrfachnennungen) & \\
Quelle: Fragebogenerhebung &
\end{tabular}

Die mit Abstand bekanntesten Initiativen aus dem Holzbereich sind die diversen Holzclusterinitiativen, die über $90 \%$ der Teilnehmer und knapp $80 \%$ der Unternehmen aus der Kontrollgruppe bekannt sind. Obwohl die Kompetenzzentren mit klaren Teilnehmerbedingungen ausgestattet sind und die Zahl der Teilnehmer in der Größenordnung von jeweils 10 liegt, erstaunt der große Bekanntheitsgrad.

\section{Tabelle 27: $\quad$ Bekanntheitsgrad anderer Initiativen [\%]}

\begin{tabular}{lrr}
\hline & Teilnehmer & Kontrollgruppe \\
\hline Kompetenzzentrum Kplus Wood & 52,38 & 29,17 \\
Holzcluster & 90,48 & 79,17 \\
Kompetenznetzwerk Knet Holz & 50,79 & 25,00 \\
industrielles Kompetenzzentrum Kind Holztechnologie & 36,51 & 29,17 \\
industrielles Kompetenzzentrum Kind Holzbau & 33,33 & 33,33 \\
\hline
\end{tabular}

Quelle: Fragebogenerhebung

\subsection{Die Rolle von Kooperationen}

Ein Ziel der Sonderaktion Holzforschung war die Stärkung von nationalen und internationalen Forschungskooperationen. Die Kooperation von Forschern verschiedener Institutionen soll für Wissensspillover sorgen und damit einen Zusatznutzen schaffen gegenüber der Situation, in der Firmen auf sich alleine gestellt sind. Wirtschaftspolitische Praktiker sehen in der Schaffung von Netzwerken und persönlichen Kontakten zwischen Akteuren per se eine gute Sache, die produktive Prozesse in Gang setzten kann. Förderprogramme haben daher meist zum Ziel, die Netzwerkbildung bei den relevanten Akteuren zu unterstützen. Dies deckt sich mit theoretischen Erwägungen, da Kooperationen und Konsortien die mit Forschungsinvestitionen verbundenen externen Effekte internalisieren können. Wie D'Aspremont und Jaquemin (1988) gezeigt haben, gilt dies allerdings nur bei hohen spillover Effekten. Bei geringen spillover Effekten ist aus sozialer Sicht die nicht-kooperative Lösung vorzuziehen, da ansonsten die negativen Monopoleffekte von Kooperationen überwiegen. Denn über eines sollte man sich bei aller Euphorie über Kooperationen stets klar sein: Sie weichen den Wettbewerb auf, und die Grenze zwischen volkswirtschaftlich fruchtbarer F\&E-Zusammenarbeit und schädlichen Kartellen ist oft schwer auszumachen.

Ein weiterer Nachteil von F\&E-Kooperationen mit mehreren Teilnehmern liegt in der Anreizstruktur, die Trittbrettfahrertum begünstigt. Jede Firma würde am liebsten von den Forschungsergebnissen des Partners profitieren ohne dabei eigene Kompetenzen offen zulegen. Wenn alle Partner so denken, ist es daher durchaus möglich, dass am Ende lediglich Forscher aus der zweiten Reihe an den Projekten teilnehmen. Das Geheimhaltungs - und daraus resultierende Anreizproblem ist jedoch nicht in 
allen Fällen von Bedeutung, es kommt stark auf die Zusammensetzung des Konsortiums (z.B. horizontale vs. vertikale Gliederung, Nichteinbindung von Konkurrenten) an.

Der folgende Abschnitt untersucht, ob und wie kooperiert wurde. Ungefähr jeder dritte Teilnehmer gab an, im Rahmen des Projektes mit mindestens einem Partner kooperiert zu haben (Tabelle 28). In der Förderdatenbank des FFF hingegen sind 43\% der Projekte als Kooperationsprojekte geführt. Der Unterschied von über 10 Prozentpunkten mag damit zusammenhängen, dass einigen Geschäftsführern, an die der Fragebogen zugegangen ist, nicht mehr alle Kooperationen bekannt waren. ${ }^{11}$

Tabelle 28: Anzahl von Kooperationspartnern [\%]

\begin{tabular}{lrrrrrrrrrrrr}
\hline Kooperationspartner & 0 & 1 & 2 & 3 & 4 & 5 & 6 & 7 & 8 & 9 & 10 & $>10$ \\
\hline Anteil & 69 & 15,5 & 7,1 & 4,4 & 0,4 & 1,3 & 0 & 0 & 0 & 0 & 1,3 & 0,8 \\
\hline
\end{tabular}

Quelle: Fragebogenerhebung

Die maximale Anzahl der Kooperationspartner betrug entsprechend unserer Fragebogenerhebung 40, die meisten Kooperationen waren allerdings eher klein und in nur ca. 8\% der Fälle waren mehr als 2 Partner beteiligt. Im Schnitt betrug die Anzahl der Kooperationspartnern 2,8. ${ }^{12}$ Die Zahl der Kooperationspartner deckt sich mit Erfahrungen aus der Organisationstheorie, die zeigen, dass eine zu hohe Teilnehmerzahl bei Projekten $\mathrm{zu}$ suboptimalen Ergebnis sen führt, da der Koordinationsaufwand mit der Teilnehmerzahl wächst und ab einer bestimmten Größe der Nutzen geringer ist als die Kosten.

Die Kooperationen haben in einem nicht unerheblichem Unfang auch $\mathrm{zu}$ neuen Netzwerken geführt: $78 \%$ der Firmen, die kooperiert haben, gaben an, dass sie den Kooperationspartner schon vorher kannten, davon haben aber die Hälfte aber noch nicht miteinander kooperiert. Der Rest ist Beziehungen mit völlig neuen Partnern eingegangen (Tabelle 29).

\section{Tabelle 29: $\quad$ Entstehung der Kooperation [\%]}

\begin{tabular}{lr}
\hline Partner kannten wir vor der Projektvorbereitung, hatten aber noch nicht kooperiert & 39 \\
Partner waren bereits früher schon Kooperationspartner & 39 \\
Partner wurden uns empfohlen & 13 \\
Partner wurde durch andere Projektpartner ins Team geholt & 9 \\
\hline
\end{tabular}

Quelle: Fragebogenerhebung

Einleitend wurde gesagt, dass die Beziehungen zwischen den teilnehmenden Firmen horizontal oder vertikal gegliedert sein können, wobei die Art der Gliederung Einfluss auf die Anreizstrukturen innerhalb des Konsortiums haben kann. Da die Sonderaktion Holzforschung stark marktnah angesiedelt war, spielt es eine große Rolle, ob es sich bei den Mitgliedern eines Verbundes um Konkurrenten oder um vertikale Elemente der Wertschöpfungskette handelt.

Nur $8 \%$ der Kooperationspartner waren direkte Mitbewerber, die überwiegende Mehrzahl der Kooperationspartner waren hingegen Zulieferer und Kunden (Tabelle

11 In der Zwischenevaluierung wurde der Anteil an Kooperationsprojekten sogar auf 75\% geschätzt (Ohler, Teischinger, Schörghofer 1998). Möglicherweise ist die Kooperationsintensität in der zwe iten Hälfte des Programms weniger stark ausgeprägt gewesen.

12 Dies ist die durchschnittliche Anzahl der Kooperationspartner für diejenigen Firmen, die überhaupt kooperiert haben. Über die gesamte Stichprobe beträgt der Wert 0,9. 
30). Das bedeutet, dass die meisten Kooperationen offenbar vertikal strukturiert waren. Dieser Aufbau ist für ein marktnahes Projekt positiv zu beurteilen, da in einem solchen Umfeld die angesprochenen Probleme der Geheimhaltung weit weniger virulent sind als bei einer horizontalen Struktur.

\begin{tabular}{lr} 
Tabelle 30: Struktur der Kooperationen [\%] & \\
\hline Zulieferer (potentielle und tatsächliche) & 25 \\
Kunden (potentielle und tatsä̈hliche) & 35 \\
indirekte Mitbewerber (mit ähnlichen Produkten auf anderen Märkten) & 1 \\
direkte Mitbewerber (mit ähnlichen Produkten auf gleichen Märkten) & 8 \\
Universitäten & 6 \\
Forschungsinstitute & 14 \\
Berater und Spezialisten & 10 \\
Sonstige & 3 \\
\hline
\end{tabular}

Quelle: Fragebogenerhebung

\subsection{Bedeutung der Programmteilnahme: Ziele und Zielerreichung}

In diesem Abschnitt werden die mit der Programmteilnahme verbundenen Ziele und das Maß der Zielerreichung diskutiert. Welchen Stellenwert hatte die Teilnahme an der Sonderaktion Holzforschung für die Teilnehmer? 53,4\% gaben an, dass das geförderte Projekt nur eine von mehreren F\&E-Aktivitäten ist. Für jeden 5. Teilnehmer $(21,9 \%)$ hingegen war es der Einstieg in die Holzforschung. Die Gruppe der Neueinsteiger ist deshalb besonders wichtig, als davon ausgegangen werden kann, dass hier der Einfluss der FFF Förderung besonders hoch ist. Ein gutes Beispiel für ein Forschungsprojekt eines solchen Neueinsteigers liefert die Firma Weinberger aus Kärnten, deren ist Geschichte kurz in nachfolgender Box dargestellt wird.

\footnotetext{
Fallbeispiel: Produktdifferenzierung mit Hilfe eines FFF Projektes

Die Firma Weinberger aus Kärnten ist bereits 1856 als Dorfsägewerk gegründet worden und hatte in der Folge eine Einschnittskapazität von $15.000 \mathrm{fm}$ Holz pro Jahr. In guten Zeiten wurde gute 2 Mio. $€$ umgesetzt. In den späten 80er Jahren hat sich ein Paradigmenwechsel in der Sägetechnik vollzogen, die neue Zerspanertechnik löste zunehmend die bis dahin vorherrschende Gattertechnik ab. Die Durchlaufgeschwindigkeit konnte durch diese Technologie deutlich erhöht werden, was zu einem starken Anstieg der Arbeitsproduktivität geführt hat. Während ein Arbeiter mit der Gattertechnik $2.500 \mathrm{fm}$ pro Jahr leistet, schafft der gleiche Arbeiter mit der neuen Technik einen Ausstoß von über $9.000 \mathrm{fm}$. Größere Sägewerke, die mit der neuen Technologie ausgestattet sind, haben mittlerweile einen Ausstoß von 800.000 fm pro Jahr. Dies ist das 8-16- fache von traditionellen Betrieben. Durch die Zerspanertechnik wird jedoch nicht nur die Produktivität des Sägeprozesses verbessert, sondern es wird durch eine wesentlich höhere Maßgenauigkeit sich auch die Qualität deutlich erhöht. Die Implikationen für die Markstruktur sind klar: Eine konstante Nachfrage kann von wesentlich weniger Herstellern bedient werden. Da die Umrüstung der Betriebe kostenintensiv ist, kommt es zu einem Konzentrationsprozess, bei dem kleinere Anbieter aus dem Markt gedrängt werden. Dementsprechend sind in den 90er Jahren zahlreiche Sägewerke vom Markt verschwunden. Heute dominieren große, finanzkräftige Konzerne wie Stora-Enso (S, SF) oder aus Österreich Mayer Melnhof oder die Bindergruppe. Die zwei einzigen Möglichkeiten für Kleinunternehmen wie Wein berger waren, sich mit anderen Werken zusammenzuschließen, oder ihre Produkte entlang der Wertschöpfungskette zu diversifizieren. Wein berger entschied sich für die letztere Strategie und versuchte, sich auf geleimte Holzbohlen zu spezialisieren. Da Mitte der 90er Jahre eine Schweizer Firma einen neuen PU Klebstoff auf den Markt gebracht hat, der absolut emissionsfrei ist, sah man gute Markt chancen bei geleimtem Holz. Der Vorteil bei geleimten Holzbohlen ist, dass sie von außen rissfrei sind, da die rissige Seite jeweils innen miteinander verklebt werden. 1996 hat man im Rahmen des FFF Projektes erforscht, wie sich die Bohlen am Besten verkleben lassen. Wichtige zu erkundende Parameter waren hier die Druck- und Witterungsverhältnisse. Das erfolgreiche Projekt hat dem Unternehmen das Überleben gesichert und mittlerweile hat es sich ganz auf Holzverleimung spezialisiert und aus dem Sägewerksgeschäft zurückgezogen. Die Produktionsanlagen wurden indessen nach Russland verkauft und
} 
stehen mittlerweile am sibirischen Baikalsee. Die verleimten Holzbohlen bieten bei hohen Margen großes Marktpotenzial, was sich in Export quote von $75 \%$ niederschlägt.

Die mit der Teilnahme verbundenen Ziele sowie deren Erreichung sind in Tabelle 31 dargestellt. ${ }^{13}$ Die drei wichtigsten Ziele waren 'Entwicklung neuer Produkte', 'Verbesserung der allgemeinen Wettbewerbsfähigkeit' sowie die 'Entwicklung neuer Prototypen'. Eine deutlich untergeordnete Rolle spielten die Punkte 'Reduktion der Mitarbeiterzahl', 'Diplomarbeiten und Dissertationen' und die 'Reorganisation des Unternehmens'. Die mit der Teilnahme verbunden Ziele sind demnach sehr produktorientiert und vorwärtsgerichtet, während die unwichtigen Ziele wie die 'Reduktion der Mitarbeiter' (z.B. durch die Entwicklung von arbeitssparender Technologie) eher defensiven Charakter haben. Dieser Befund ist positiv zu beurteilen.

Die drei wesentlichen Programmziele des FFF, nämlich (i) Verbesserung der Forschungssituation, (ii) Beseitigung von Innovationshemmnissen und (iii) Stärkung der Wettbewerbsfähigkeit bilden sich auch in den Zielen der Teilnehmer ab. Eine deutlich geringere Entsprechung findet jedoch das Programmziel 'Stärkung von nationalen und internationalen Forschungskooperationen'. Kooperationen betreffende Punkte wie 'Vertiefung von Kooperationen zu Lieferanten und Kunden', 'Erfahrung sammeln bei Kooperationen' und 'Risikoteilung mit Projektpartnern' sind den Teilnehmern nur mäßig wichtig gewesen. Insgesamt unterstreichen die Zielsetzungen der Teilnehmer den anwendungsbezogenen Charakter des Programms, bei dem akademische Ziele wie Publikationen oder Dissertationen keine Rolle spielen. Ein wenig überraschend ist die relativ niedrige Priorität bei dem Punkt 'Zugang zu neuen Finanzierungsquellen'. Die Teilnehmer verbinden mit einer Teilnahme an der Sonderaktion Holzforschung vermutlich in erster Linie Lerneffekte und gar nicht so sehr die direkten finanziellen Unterstützungen.

Aus der Tabelle 31 lässt sich auch der Grad der Zielerreichung ablesen, der durch vier Kategorien abgebildet wird: Ziel wurde (i) innerhalb eines Jahres, (ii) innerhalb von 3 Jahren, (iii) später, (iv) gar nicht erreicht. Alles in allem wurden die mit dem Projekt verbundenen Ziele zu über 90\% erreicht (letzte Spalte), meist bereits schon im ersten Jahr.

13 Bedeutsame Ziele sind hier diejenigen Ziele, die von den Respondenten als sehr wichtig oder wichtig eingestuft wurden. 
Tabelle 31: Bedeutende Ziele und deren Erreichung [absolute Häufigkeiten]

\begin{tabular}{|c|c|c|c|c|c|c|}
\hline & \multirow[t]{2}{*}{ Ziel } & \multicolumn{5}{|c|}{ Zielerreichung } \\
\hline & & $<1 \mathrm{~J}$. & $<3 \mathrm{~J}$ & $>3 \mathrm{~J}$. & $\begin{array}{r}\text { gar } \\
\text { nicht }\end{array}$ & $\begin{array}{r}\text { Erreichung } \\
{[\%]}\end{array}$ \\
\hline Entwicklung neuer Produkte & 58 & 24 & 24 & 8 & 2 & $97 \%$ \\
\hline $\begin{array}{l}\text { Verbesserung der allgemeinen } \\
\text { Wettbewerbsfähigkeit }\end{array}$ & 56 & 14 & 28 & 10 & 4 & $93 \%$ \\
\hline Entwicklung von Prototypen & 49 & 29 & 17 & 2 & 1 & $98 \%$ \\
\hline $\begin{array}{l}\text { Entwicklung neuer } \\
\text { Produktionstechnologien }\end{array}$ & 42 & 14 & 22 & 4 & 2 & $95 \%$ \\
\hline $\begin{array}{l}\text { Vertiefung bestehender } \\
\text { Anwendungskenntnisse }\end{array}$ & 40 & 18 & 19 & 2 & 1 & $98 \%$ \\
\hline Verbesserung existierender Produkte & 38 & 24 & 10 & 2 & 2 & $95 \%$ \\
\hline $\begin{array}{l}\text { Verbesserung existierender } \\
\text { Produktionstechnologien }\end{array}$ & 38 & 16 & 15 & 6 & 1 & $97 \%$ \\
\hline interner Kompetenzaufbau & 38 & 15 & 20 & 2 & 1 & $97 \%$ \\
\hline $\begin{array}{l}\text { Unterstützung der Qualifikation von } \\
\text { Mitarbeitern }\end{array}$ & 31 & 14 & 14 & 2 & 1 & $97 \%$ \\
\hline $\begin{array}{l}\text { Zugang zu zusätzlichen } \\
\text { Finanzierungsquellen }\end{array}$ & 30 & 14 & 10 & 0 & 6 & $80 \%$ \\
\hline Patentanmeldungen & 28 & 13 & 8 & 4 & 3 & $89 \%$ \\
\hline $\begin{array}{l}\text { Adaption neuer Verfahren auf eigenen } \\
\text { Bedarf }\end{array}$ & 28 & 9 & 14 & 2 & 3 & $89 \%$ \\
\hline $\begin{array}{l}\text { Vertiefung von Kooperationen zu } \\
\text { Lieferanten/Kunden }\end{array}$ & 27 & 14 & 8 & 3 & 2 & $93 \%$ \\
\hline Benchmarking ('Wo stehen wir?') & 24 & 9 & 11 & 3 & 1 & $96 \%$ \\
\hline $\begin{array}{l}\text { Zugang zu Labors, Test- und } \\
\text { Prüfeinrichtungen }\end{array}$ & 22 & 15 & 5 & 0 & 2 & $91 \%$ \\
\hline Erfahrung sammeln bei Kooperationen & 21 & 9 & 8 & 3 & 1 & $95 \%$ \\
\hline Publikationen & 20 & 11 & 8 & 1 & 0 & $100 \%$ \\
\hline Risikoteilung mit Projektpartnern & 18 & 10 & 3 & 2 & 3 & $83 \%$ \\
\hline Einstellung neuer Mitarbeiter & 16 & 10 & 5 & 0 & 1 & $94 \%$ \\
\hline Entwicklung von Normen und Standards & 11 & 3 & 3 & 2 & 3 & $73 \%$ \\
\hline Reorganisation des Unternehmens & 10 & 2 & 6 & 2 & 0 & $100 \%$ \\
\hline Diplomarbeiten/Dissertationen & 8 & 6 & 1 & 0 & 1 & $88 \%$ \\
\hline Reduktion der Mitarbeiterzahl & 2 & 0 & 1 & 0 & 1 & $50 \%$ \\
\hline
\end{tabular}

Quelle: Fragebogenerhebung

Um zu überprüfen, ob Ziele, die als wichtig eingestuft werden, auch schneller erreicht werden, wurde in Tabelle 32 eine mittlere Zielerreichung gebildet, die folgendermaßen zu lesen ist. Diejenige Firma, für die das Ziel 'Entwicklung von Prototypen' wichtig bzw. sehr wichtig war, hat dieses Ziel im Schnitt (Median) innerhalb eines Jahres erreicht. Solche Firmen, für die dieses Ziel unwichtig bzw. sehr unwichtig war, hat dieses (unwichtige) Ziel im Schnitt zwar auch erreicht, jedoch erst nach drei Jahren oder später. Das Ergebnis, dass wichtige Ziele schneller erreicht wurden als unwichtige Ziele, mag etwas unspektakulär klingen, zeigt aber doch, dass die Teilnehmer sehr zielorientiert gearbeitet haben. Relevanz fokussiert also die Aufmerksamkeit. 
Tabelle 32: Mittlere Zielerreichung bei bedeutsamen und unbedeutsamen Zielsetzungen

\begin{tabular}{|c|c|c|}
\hline & $\begin{array}{l}\text { unwichtige und eher } \\
\text { unwichtige Ziele }\end{array}$ & $\begin{array}{l}\text { sehr wichtig und } \\
\text { wichtige Ziele }\end{array}$ \\
\hline Entwicklung von Prototypen & später & $<1 \mathrm{Jahr}$ \\
\hline Verbesserung existierender Produkte & später & $<1 \mathrm{Jahr}$ \\
\hline Publikationen & gar nicht & $<1 \mathrm{Jahr}$ \\
\hline Diplomarbeiten/Dissertationen & gar nicht & $<1 \mathrm{Jahr}$ \\
\hline Vertiefung von Kooperationen zu Lieferanten/Kunden & gar nicht & $<1 \mathrm{Jahr}$ \\
\hline Zugang zu Labors-, Test- und Prüfeinrichtungen & gar nicht & $<1 \mathrm{Jahr}$ \\
\hline Risikoteilung mit den Projektpartnern & gar nicht & $<1 \mathrm{Jahr}$ \\
\hline Einstellung neuer Mitarbeitern & gar nicht & $<1 \mathrm{Jahr}$ \\
\hline Entwicklung neuer Produkte & gar nicht & $<3$ Jahre \\
\hline Entwicklung neuer Produkttechnologien/Verfahren & gar nicht & $<3$ Jahre \\
\hline Verbesserung existierender Technologien/Verfahren & gar nicht & $<3$ Jahre \\
\hline Vertiefung bestehender Anwendungskenntnisse & $<3$ Jahren & $<3$ Jahre \\
\hline Adaption neuer Verfahren auf eigenen Bedarf & gar nicht & $<3$ Jahre \\
\hline Entwicklung von Normen und Standards & gar nicht & $<3$ Jahre \\
\hline Patentanmeldungen & gar nicht & $<3$ Jahre \\
\hline Unterstützung der Qualifikation von Mitarbeitern & gar nicht & $<3$ Jahre \\
\hline Erfahrungen sammeln bei Kooperationsprojekten & später/gar nicht & $<3$ Jahre \\
\hline Benchmarking ('Wo stehen wir?') & gar nicht & $<3$ Jahre \\
\hline Zugang zu zusätzlichen Finanzierungsquellen & gar nicht & $<3$ Jahre \\
\hline Reorganisation des Unternehmens & gar nicht & $<3$ Jahre \\
\hline interner Kompetenzaufbau & gar nicht & $<3$ Jahre \\
\hline Verbesserung der allgemeinen Wettbewerbsfähigkeit & keine Angabe & $<3$ Jahre \\
\hline Reduktion der Mitarbeiterzahl & gar nicht & später \\
\hline
\end{tabular}

Quelle: Fragebogenerhebung

Eine wichtige Bemerkung noch zum Ziel 'Reduktion der Mitarbeiterzahl'. Es hat nicht nur keine Rolle gespielt, sondern die teilnehmenden Firmen haben im Zuge der geförderten Projekte ganz im Gegenteil sogar 273 neue Mitarbeiter eingestellt. Hochgerechnet auf die Grundgesamtheit bedeutet das, das ca. 500 neue Arbeitsplätze entstanden sind.

\subsection{Schwierigkeiten im Projektverlauf}

Werfen wir nun einen Blick auf die im Projektverlauf entstandenen Schwierigkeiten. Folgende Probleme sind in erster Linie aufgetreten (Tabelle 33): (i) Die Problemstellung war schwieriger als erwartet, (ii) Zeit und Kosten wurden unterschätzt, (iii) Projektmitarbeiter waren zu sehr mit dem Tagesgeschäft beschäftigt.

Die Tatsache, dass die Schwierigkeiten von vielen unterschätzt wurden, deutet darauf hin, dass viele Firmen Erfahrungsdefizite im F\&E-Bereich haben. Um zu überprüfen, inwiefern Erfahrung die perzipierten Schwierigkeiten beeinflusst, haben wir die Teilnehmer in zwei Gruppen - F\&E-Einsteiger und F\&E-Profis - eingeteilt $^{14}$. Hier zeigt sich, dass in der Tat die Schwierigkeiten von den Einsteigern eher unterschätzt wurden als von den Versierten: Während 93\% der Einsteiger die technische Problemstellung unterschätzt haben, betrug der Anteil bei den Versierten lediglich 73\%. Entsprechend gaben $81 \%$ der Einsteiger an, Zeit und Kosten falsch eingeschätzt zu haben, bei den Versierten waren dies nur 68\%. Mit dem Problem,

14 Profis sind diejenigen Firmen, die mehrere F\&E-Projekte gleichzeitig durchführen. Einsteiger sind diejenigen, bei denen die Teilnahme an der 'Sonderaktion' der Einstieg in die Holzforschung darstellte. 
dass die Mitarbeiter zu sehr mit dem Tagesgeschäft zu tun hatten, kämpften hingegen eher die Versierten. Der Anteil lag hier bei $60 \%$ für die Versierten und lediglich 46\% für die Einsteiger. Dies zeigt, dass die Einsteiger zwar Schwierigkeiten hatten, die Komplexität des Projektes richtig einzuschätzen, das Projekt scheint andererseits aber eher im Mittelpunkt der Aufmerksamkeit gestanden zu sein als bei den Versierten.

Tabelle 33: Im Rahmen des Projektverlaufs eingetretene Schwierigkeiten und deren Lösung [absolute Häufigkeiten]

\begin{tabular}{|c|c|c|c|c|c|c|}
\hline & \multirow{2}{*}{$\begin{array}{l}\text { einge- } \\
\text { treten }\end{array}$} & \multicolumn{4}{|c|}{ Problemstärke } & \multirow{2}{*}{$\begin{array}{l}\text { Problem } \\
\text { gelöst }\end{array}$} \\
\hline & & $\begin{array}{l}\text { großes } \\
\text { Problem }\end{array}$ & $\begin{array}{l}\text { geringes } \\
\text { Problem }\end{array}$ & $\begin{array}{c}\text { kein } \\
\text { Problem }\end{array}$ & k.A. & \\
\hline $\begin{array}{l}\text { technische Problemstellung } \\
\text { war schwieriger als erwartet }\end{array}$ & $\begin{array}{r}55 \\
(77,5 \%)\end{array}$ & 20 & 28 & 2 & 5 & $40(73 \%)$ \\
\hline $\begin{array}{l}\text { Zeit- und Kosten wurden } \\
\text { unterschätzt }\end{array}$ & $\begin{array}{r}53 \\
(72,6 \%)\end{array}$ & 21 & 24 & 4 & 4 & $28(53 \%)$ \\
\hline $\begin{array}{l}\text { Projektmitarbeiter waren zu } \\
\text { sehr mit Tagesgeschäften } \\
\text { beschäftigt }\end{array}$ & $\begin{array}{r}40 \\
(55,6 \%)\end{array}$ & 11 & 25 & 4 & 0 & $20(50 \%)$ \\
\hline $\begin{array}{l}\text { mangelnde Kenntnis } \\
\text { geeigneter } \\
\text { Kooperationspartner }\end{array}$ & $\begin{array}{r}22 \\
(31,0 \%)\end{array}$ & 5 & 11 & 3 & 3 & $7(32 \%)$ \\
\hline $\begin{array}{l}\text { qualifiziertes Personal war } \\
\text { nicht ausreichend verfügbar }\end{array}$ & $\begin{array}{r}18 \\
(25,0 \%)\end{array}$ & 7 & 10 & 1 & 0 & $10(56 \%)$ \\
\hline $\begin{array}{l}\text { Partner bringen zu wenig } \\
\text { ein (Trittbrettfahrer) }\end{array}$ & $\begin{array}{r}17 \\
(23,9 \%)\end{array}$ & 7 & 5 & 3 & 2 & $8(47 \%)$ \\
\hline $\begin{array}{l}\text { Offenlegung von } \\
\text { Innovations- und } \\
\text { Produktstrategien gegenüber } \\
\text { Dritten }\end{array}$ & $\begin{array}{r}16 \\
(22,5 \%)\end{array}$ & 2 & 5 & 9 & 0 & $1(6 \%)$ \\
\hline $\begin{array}{l}\text { Änderungen der eigenen } \\
\text { Zielsetzungen }\end{array}$ & $\begin{array}{r}16 \\
(22,5 \%)\end{array}$ & 2 & 9 & 5 & 0 & $5(31 \%)$ \\
\hline $\begin{array}{l}\text { Änderungen der Ziele bei } \\
\text { den Partner }\end{array}$ & $\begin{array}{r}15 \\
(21,1 \%)\end{array}$ & 9 & 3 & 2 & 1 & $7(47 \%)$ \\
\hline $\begin{array}{l}\text { Fluktuation im eigenen } \\
\text { Projektteam }\end{array}$ & $\begin{array}{r}13 \\
(18,1 \%)\end{array}$ & 5 & 5 & 3 & 0 & $7(54 \%)$ \\
\hline $\begin{array}{l}\text { Schwierigkeiten bei der } \\
\text { Geheimhaltung gemeinsam } \\
\text { erzielter Ergebnisse }\end{array}$ & $\begin{array}{r}12 \\
(16,4 \%)\end{array}$ & 4 & 3 & 4 & 1 & $3(25 \%)$ \\
\hline $\begin{array}{l}\text { Projektmanagement hat } \\
\text { nicht funktioniert }\end{array}$ & $\begin{array}{r}11 \\
(15,3 \%)\end{array}$ & 4 & 5 & 2 & 0 & $4(36 \%)$ \\
\hline $\begin{array}{l}\text { Fluktuation in den } \\
\text { Projektteams der Partner }\end{array}$ & $7(9,9 \%)$ & 1 & 3 & 3 & 0 & $3(43 \%)$ \\
\hline $\begin{array}{l}\text { Fragestellung des Projektes } \\
\text { hat sich als überholt } \\
\text { erwiesen }\end{array}$ & $5(6,9 \%)$ & 2 & 0 & 3 & 0 & $1(20 \%)$ \\
\hline
\end{tabular}

Quelle: Fragebogenerhebung

Kaum Probleme gab es mit dem Projektmanagement. Hier wurde in den Gesprächen auch die Unterstützung des FFF erwähnt, der auf eine zügige Abwicklung des Projektes geachtet hat. Eher im Mittelfeld rangiert auch die Sorge vor einer Offenlegung von Forschungsergebnissen. In anderen Branchen wie der Halbleiterindustrie mit extrem kurzen Produktzyklen ist dies ein ernstes Problem und hält viele Firmen von einer Teilnahme an öffentlichen Programmen ab (Brandstetter, Sakihabara 1998). Im Vergleich dazu ist die Holzforschung doch eher bodenständig. Es wirft aber auch ein positives Licht auf den FFF, dem offenbar Diskretion zugetraut wird. 
Ebenfalls aus Tabelle 33 ist ersichtlich, dass die Schwierigkeiten grosso modo in ca. $50 \%$ der Fälle gelöst werden konnten. Gerade bei den Schwierigkeiten, die besonders häufig aufgetreten sind, liegt die Lösungsquote auch besonders hoch. So haben $73 \%$ der Unternehmen, für die es die technische Fragestellung schwieriger als erwartet war, das Problem im laufe des Projektes gelöst. Ebenfalls konnten in 56\% der Fälle die Probleme gelöst werden, die sich aus der falschen Einschätzung der Projektkosten und Zeit ergeben haben. Hohe Lösungsquoten gab es außerdem bei der fehlenden Verfügbarkeit von qualifiziertem Personal (56\%) bzw. der Fluktuation im Projektteam (54\%). Die niedrige Lösungsquote bei dem Punkt 'mangelnde Kenntnis geeigneter Kooperationspartner' überrascht etwas, da der FFF hier sicher Hilfestellung hätte geben können und sicherlich auch gemacht hätte, sofern man diesbezüglich auf ihn zukommen wäre.

\subsection{Kommerzielle Verwertung und Fortsetzung der Forschungstätigkeiten}

Eines der wesentlichen Ziele bei marktnahen Forschungsprojekten ist natürlich die rasche kommerzielle Umsetzung der Projektergebnisse und die Realisierung von Gewinnen. Eher längerfristige Wirkungen wie ein allgemeiner Kompetenzaufbau und eine Erhöhung der Absorptionskapazität für externes Wissen sind zwar nicht minder wichtig, doch ganz unmittelbar lassen sich die Projektergebnisse am Besten durch die neu generierten Umsätze beurteilen. Hier kann man sehen, dass die Projekte sehr marktnah waren. Das wird dadurch unterstrichen, dass ca. 80\% der befragten Teilnehmer entweder bereits mit der kommerziellen Umsetzung der Projektergebnisse begonnen haben bzw. konkrete Verwertungspläne ausgearbeitet haben (Tabelle 34).

\section{Tabelle 34: Kommerzielle Verwertung der Ergebnisse [\%]}

es existieren noch keine Verwertungspläne $\quad 18,2$

es existieren Verwertungspläne, aber mit der Verwertung wurde noch nicht begonnen $\quad 16,9$

es wurde bereits mit der kommerziellen Verwertung begonnen $\quad 54,5$

keine Angaben

10,4

Quelle: Fragebogenerhebung

Für 73\% der Firmen, die bereits mit der Verwertung begonnen haben, vergingen nicht mehr als drei Jahre zwischen Projektbeginn und der Kommerzialisierung der Forschungsergebnisse (Tabelle 35). Dies ist unserer Erfahrung nach relativ schnell, bei anderen von Technopolis evaluierten Programmen ist diese Zeitspanne von Projektbeginn bis zur Kommerzialisierung mitunter deutlich länger.

\begin{tabular}{lc} 
Tabelle 35: & $\begin{array}{c}\text { Zeitlicher Abstand zwischen Projektbeginn und kommerzieller } \\
\text { Verwertung [\%] }\end{array}$ \\
\hline < 1 Jahr & 13,3 \\
< 2 Jahre & 28,9 \\
< 3 Jahre & 31,1 \\
< 4 Jahre & 8,9 \\
< 5 Jahre & 2,2 \\
< 6 Jahre & 2,2 \\
< 7 Jahre & 2,2 \\
keine Angaben & 11,1 \\
\hline Quelle: Fragebogenerhebung
\end{tabular}


Die langfristigen Wirkungen des Programms wie der Aufbau von F\&E-Kapazitäten sind etwas schwieriger zu quantifizieren. Nichtsdestotrotz haben wir die Teilnehmer nach ihren Plänen nach Projektende gefragt, um gewisse Rückschlüsse auf die Nachhaltigkeit des Programms ziehen zu können. Hier zeigt sich, dass lediglich 15\% der Teilnehmer nach Projektablauf die Forschungstätigkeiten in diesem Bereich wieder einstellen. Der Rest möchte weitermachen und die Holzforschungsaktivitäten teilweise sogar noch verstärken, zur Hälfte allerdings nur mit neuen Fördermitteln (Tabelle 36). Während die eine Erkenntnis erfreulich ist, lässt die andere Zweifel aufkommen. Es ist in diesem Zusammenhang nur zu hoffen, dass sich hier keine Subventionsmentalität breit macht, bei der öffentliche Förderungen und Subventionen mit einer Selbstverständlichkeit eingemahnt - und bedient - werden, vor der kritische Beobachter immer wieder warnen (Olson 1992).

\section{Tabelle 36: Pläne nach Projektende [\%]}

\begin{tabular}{lr}
\hline die F\&E-Aktivitäten in diesem Bereich mit Förderungen fortsetzen & 49,3 \\
die kommerzielle Verwertung der Projektergebnisse vorantreiben & 45,1 \\
die F\&E-Aktivitäten in diesem Bereich mit eigenen Mitteln fortsetzen & 42,3 \\
gemeinsam mit Partnern F\&E-Aktivitäten auf andere Themen beginnen & 31,0 \\
alleine F\&E-Aktivitäten auf andere Themen beginnen & 28,2 \\
Projektteam auflösen und F\&E-Aktivitäten in diesem Bereich beenden & 15,5 \\
die F\&E-Aktivitäten in diesem Bereich fortsetzen, jedoch mit anderen Partnern & 8,5 \\
Gründung eines Joint/Ventures / spin-off Unternehmens & 4,2 \\
\hline (Mehrfachnennungen) & \\
Quelle: Fragebogenerhebung &
\end{tabular}

\subsection{Kosten und Nutzen der Programmteilnahme}

Kommen wir nun zur Einschätzung des Kosten-Nutzen Verhältnisses der Programmteilnahme. Diese Frage testet sozusagen den Wohlfühlfaktor der beteiligten Unternehmen. Hier gilt, dass ein hohe Zufriedenheit allenfalls eine notwendige, nicht jedoch eine hinreichende Bedingung für ein gutes Programm ist. Es ist schließlich nicht der alleinige Zweck, die Zufriedenheit der Firmen zu bedienen, sondern positive volkswirtschaftliche Effekte zu erzielen (siehe Kapitel 6).

Wenn man das Verhältnis aus Kosten und Nutzen aufspaltet, so ergibt sich der Nutzen aus der Programmteilnahme aus der Förderung und aus Lerneffekten, die durch die Projektbetreuung des FFF resultieren. Auf der Kostenseite ist in erster Linie die Abwicklung der Förderung zu nennen (Antragstellung, Dokumentation etc.), deren Kosten im Prinzip leicht in Arbeitsstunden bzw. in Geld zu beziffern. Wenn die Höhe der Förderung geringer ist als die Arbeitskosten für die Antragstellung und Abwicklung, so ist die Teilnahme am Programm aus der ex post Sicht der Firma ein Fehler gewesen, und nur weil ex ante Unklarheit über Kosten und Nutzen herrschte, hat man sich überhaupt um eine Teilnahme beworben. Da bei hohen Externalitäten des Forschungsprojekts nichtsdestotrotz positive gesamtwirtschaftliche Effekte induziert werden können, ist die private Sicht der Unternehmen allerdings nicht die letztendliche Richtschnur, an der ein Programm bewertet werden soll.

Wie beurteilen nun die Teilnehmer die Sonderaktion Holzforschung? Für $2 / 3$ der Teilnehmer hat der Nutzen der Teilnahme an der Sonderaktion die damit verbundenen Kosten überwogen (Tabelle 37). Dies sieht auf dem ersten Blick nicht überragend aus, ist im Vergleich mit anderen Programmen aber eher hoch. Bei der von Technopolis durchgeführten Evaluierung des deutschen Förderprogramms 
Mikrosystemtechnik gaben gerade einmal 51\% der beteiligten Unternehmen an, dass das Programm einen Nettonutzen gestiftet hat.

Tabelle 37: Kosten-Nutzen-Einschätzung der Programmteilnahme durch die Förderteilnehmer [\%]

\begin{tabular}{lr}
\hline Kosten sehr viel höher als Nutzen & 10,4 \\
Kosten höher als Nutzen & 7,8 \\
Kosten ein wenig höher als Nutzen & 7,8 \\
Kosten gleich Nutzen & 7,8 \\
Nutzen ein wenig höher als Kosten & 11,7 \\
Nutzen höher als Kosten & 16,9 \\
Nutzen sehr viel höher als Kosten & 29,9 \\
keine Angaben & 7,8 \\
\hline
\end{tabular}

Quelle: Fragebogenerhebung

Die Einschätzung des Nettonutzens ist übrigens unabhängig von der F\&E-Erfahrung der Teilnehmer. Wir haben die Gruppe der Einsteiger mit der Gruppe der Profis miteinander verglichen und keine signifikanten Unterschiede feststellen können. ${ }^{15}$ Erwartet haben wir abnehmende Grenzkosten einer Bewerbung und daher ein besseres Kosten-Nutzen Verhältnis bei den Profis, die oft schon Erfahrungen mit geförderten F\&E-Projekten gemacht haben dürften.

\subsection{Bewertung der administrativen Programmabwicklung}

Das Kapitel über den Einschätzungen des Programms soll abgeschlossen werden mit der Bewertung der administrativen Projektabwicklung von dem Zeitpunkt der Bewerbung bis hin zu der Dokumentation der Projektergebnisse. Dieser Punkt steht im engen Zusammenhang mit den Kosten einer Programmteilnahme, die umso höher sind, je aufwendiger und komplizierter die Bewerbungs- und Abwicklungsmodalitäten sind. Im Allgemeinen bekommt der FFF von den Teilnehmern durchwegs gute bis sehr gute Noten (Tabelle 38, die häufigsten Nennungen sind jeweils fett hervorgehoben). Besonders zufrieden ist man mit der Geschwindigkeit der Abwicklung und der Zugänglichkeit zu Informationen. Erwartungsgemäß haben die abgelehnten Bewerbern durchgängig eine schlechtere Meinung von den Leistungen des FFF. Es ist allerdings anzunehmen, dass hier eine gewisse Verbitterung hinein spielt.

Tabelle 38: Benotungen der Administration [\%]

\begin{tabular}{l|rr|rr|rr|r}
\cline { 2 - 7 } & \multicolumn{2}{|c|}{$\begin{array}{c}\text { Geschwindigkeit der } \\
\text { Abwicklung }\end{array}$} & \multicolumn{2}{c|}{$\begin{array}{c}\text { Nachvollziehbarkeit der } \\
\text { Entscheidung }\end{array}$} & \multicolumn{2}{c|}{$\begin{array}{c}\text { Zugänglichkeit von } \\
\text { Informationen }\end{array}$} & $\begin{array}{c}\text { Berichts- und } \\
\text { Kontroll- } \\
\text { aufwand }\end{array}$ \\
\cline { 2 - 8 } & Teilnehmer & Abgelehnte & Teilnehmer & Abgelehnte & Teilnehmer & Abgelehnte & Teilnehmer \\
\hline sehr gut & $\mathbf{4 0 , 3}$ & 20,0 & $\mathbf{3 1 , 2}$ & 0,0 & $\mathbf{4 4 , 2}$ & 20,0 & 19,5 \\
gut & 33,8 & $\mathbf{4 0 , 0}$ & 20,8 & $\mathbf{4 0 , 0}$ & 31,2 & 20,0 & 19,5 \\
neutral & 15,6 & 0,0 & $\mathbf{3 1 , 2}$ & 0,0 & 13,0 & 20,0 & $\mathbf{2 6 , 0}$ \\
schlecht & 3,9 & 40,0 & 10,4 & $\mathbf{4 0 , 0}$ & 7,8 & $\mathbf{4 0 , 0}$ & $\mathbf{2 6 , 0}$ \\
sehr schlecht & 1,3 & 0,0 & 2,6 & 20,0 & 3,0 & 0,0 & 3,9 \\
k.A. & 5,2 & 0,0 & 3,9 & 0,0 & 3,9 & & \\
\hline
\end{tabular}

Quelle: Fragebogenerhebung

15 Profis sind diejenigen Firmen, die mehrere F\&E-Projekte gleichzeitig durchführen. Einsteiger sind diejenigen, bei denen die Teilnahme an der 'Sonderaktion' der Einstieg in die Holzforschung darstellte. 


\section{Additionalitäten}

Die Frage nach den Additionalität eines Programms nimmt einen entscheidenden Stellenwert bei einer Evaluierung ein. In diesem Abschnitt soll noch einmal kurz dargelegt werden, warum dies sozusagen die Gretchenfrage ist.

Das klassische Argument der Ökonomen lautet, dass Firmen ohne öffentliche Unterstützung nicht genug in F\&E investieren würden, da sie die Erträge aus ihren F\&EInvestitionen nicht exklusiv nutzen können. Da F\&E gesellschaftlich wünschenswert sind und den zentralen Bestimmungsfaktor für Produktivitäts- und Wirtschaftswachs tum darstellen, sollte die öffentliche Hand die Anreize für F\&E-Investitionen erhöhen. Studien kommen immerhin zu jährlichen volkswirtschaftlichen Ertragsraten von F\&E-Investitionen von bis zu 50\% (Tabelle 39), weshalb F\&E-Aktivitäten aus gesellschaftlicher Perspektive eine lohnende Investition sind. Ausnahmen sind hier duplikative F\&E-Ausgaben (sogenannte Patentrennen), bei denen mehrere Firmen an einem Problem forschen, aber letztendlich nur ein Gewinner die Früchte der Forschungsausgaben ernten kann (siehe z.B. Reinganum 1982). In einer solchen Situation, die man oftmals in High-Tech Branchen vorfindet, mag es sinnvoll sein, verschiedene Unternehmen in einem Konsortium zusammenzufassen, damit die aggregierten Forschungsausgaben sinken. Amerikanische und japanische Konsortien im Halbleiterbereich haben z.B. erfolgreich dazu beigetragen, volkswirtschaftlich sinnlose F\&E-Investitionen zu verringern (Brandstetter, Sakihabara 1998, Irwin, Klenow 1996). Additionalität ist also nicht per se gut, sondern kontextabhängig. Im Fall der Sonderaktion Holzforschung ist allerdings davon auszugehen, dass eine Erhöhung der privaten F\&E-Ausgaben angestrebt wurde und es eher wenig Indizien dafür gibt, dass sich die österreichische Holzindustrie einen F\&E-Wettlauf liefert, den man Eindämmen müsste.

Tabelle 39: $\quad$ Ertragsraten auf F\&E-Ausgaben

\begin{tabular}{|c|c|c|c|}
\hline Firmenebene & & Branchenebene & \\
\hline Autoren & $\begin{array}{r}\text { Ertragsrate } \\
{[\%]}\end{array}$ & Autoren & $\begin{array}{r}\text { Ertragsrate } \\
{[\%]}\end{array}$ \\
\hline Minasian (1969) & 54 & Scherer $(1982,1984)$ & 36 \\
\hline Schankerman (1981) & 49 & Bernstein, Nadiri (1991) & 22 \\
\hline Griliches (1986) & 36 & Griliches (1980) & 21 \\
\hline Griliches, Mairesse (1986) & 33 & Link (1978) & 19 \\
\hline Griliches, Mairesse (1984) & 30 & Bernstein, Nadiri (1988) & 19 \\
\hline Mansfield (1980) & 28 & Sveikauskas (1981) & 16 \\
\hline Griliches (1980) & 27 & Wolff, Nadiri (1987) & 15 \\
\hline Jaffe (1986) & 25 & Terleckyj (1974) & 15 \\
\hline Clark, Griliches (1984) & 19 & Mohnen, Nadiri, Prucha (1986) & 11 \\
\hline Griliches, Mairesse (1983) & 19 & Patel, Soete (1988) & 6 \\
\hline Bernstein, Nadiri (1989) & 15 & Griliches, Lichtenberg (1984) & 4 \\
\hline Lichtenberg, Siegel (1991) & 13 & & \\
\hline Schankerman, Nadiri (1986) & 13 & & \\
\hline Bernstein, Nadiri (1989) & 7 & & \\
\hline
\end{tabular}

Quelle: Nadiri (1993)

Akzeptiert man den Befund, dass es das wirtschaftspolitische Ziel war, die F\&EAnreize in der Holzindustrie zu erhöhen, so können für die Erreichung dieses Zieles verschiedene Instrumente eingesetzt werden. Subventionen (entweder über das 
Steuersystem oder als direkter Geldtransfer) ist hier nur ein Instrument von mehreren. Andere Ansatzpunkte sind hier die Stärkung des Patentrechts oder die Stimulierung der Nachfrage nach innovativen Produkten, beispielsweise im Rahmen von öffentlichen Beschaffungsprogrammen (Porter 1990).

Der Nachteil von Forschungssubventionen ist, dass in der Praxis deren optimale Höhe nur schwer zu bestimmen ist. Meist arbeiten die Förderinstitutionen daher mit Faustregeln, die z.B. besagen, dass 50\% der Kosten eines F\&E-Projekts übernommen werden. Dabei kann es vorkommen, dass durch die Subvention keine zusätzliche Investition ausgelöst wird sondern lediglich die Finanzierung einer ohnehin geplanten Investition auf die öffentliche Hand überwälzt wird (crowding out). In diesem Zusammenhang muss auch erwähnt werden, dass eine Förderung nur dann zu mehr F\&E führen kann, wenn es ein hinreichend elastisches Angebot an Inputfaktoren gibt, die im Forschungsprozess eine Rolle spielen. Wenn es z. B. ein Engpass an Ingenieuren auf dem Arbeitsmarkt gibt, so kann kurzfristig selbst eine Förderungen nicht zu einer Erhöhung der Forschungsleistung beitragen. In einer solchen Situation würde es lediglich einen Preiseffekt geben, der sich in höheren Gehältern der Forscher niederschlägt. Ein Förderprogramm, das beispielsweise im Jahr 2000 auf die Internetbranche abzielte, hätte mit Sicherheit nicht die erwünschten Effekte gehabt und stattdessen lediglich dazu beigetragen, den Markt weiter zu überhitzen. Wie wir im letzten Abschnitt gesehen haben, wird die Verfügbarkeit von qualifiziertem Personal nicht als sehr großes Problem angesehen (Tabelle 33). Insofern ist die Holzbranche ein guter Adressat für ein Forschungsförderungsprogramm.

Es gibt mehrere Möglichkeiten, ein Programm auf Mitnahmeeffekte hin zu untersuchen:

- Fragebogenerhebung. Im Rahmen von standardisierten Fragebögen werden die verantwortlichen Manager direkt gefragt, ob sie das geförderte Projekt auch in Abwesenheit von Förderung durchgeführt hätten. Hierbei muss aber mit strategischem Antwortverhalten gerechnet werden, da es im Sinne des Managers ist, dass die Programmevaluierung gut ausfällt und dadurch weitere Fördergelder gesichert werden (vgl. Klette, Moen, Griliches 2000). Die Additionalität dürfte bei dieser Methode daher systematisch höher geschätzt werden.

- Fallstudien und Interviews. Auch hier ist das Problem des strategischen Antwortverhaltens evident. Außerdem sind Fallstudien nicht in dem Sinn repräsentativ wie es Fragebogenerhebungen sein können und die Ergebnisse von den Wahrnehmungen und (Vor)Urteilen des Interviewers abhängig. Es ist nicht auszuschließen, dass der Interviewer seine Interpretations spielräume dazu nutzt, das Programm in einem guten oder schlechten Licht erscheinen zu lassen. Hinzu kommen die hohen Grenzkosten pro Fall, so dass Fallstudien nur in Ergänzung $\mathrm{zu}$ anderen Methoden angewendet werden sollten.

- Ökonometrische Analyse. Statistische Analyseverfahren, die sich auf Firmenoder Branchendaten stützen, ist die vorherrschende Methode in der wissenschaftlichen Diskussion. Der Vorteil ist hier die größere Objektivität ${ }^{16}$, die aber zumeist durch eine enge Fragestellung erkauft wird und nicht auf das geförderte Projekt und dessen unmittelbares Wirkungsumfeld abstellt. Dazu kommt, dass die verfügbare Methode öfter die Fragestellung prägt als umgekehrt.

16 Dabei wird jedoch häufig vergessen, dass die 'objektiven' Fakten nicht selten über Fragebögen ermittelt werden (s.o.). 


\subsection{Fragebogenauswertung und Interviews}

Im Rahmen des Befragung wurde den Teilnehmern die Frage gestellt, ob sie das F\&E-Projekt auch ohne die Förderung durchgeführt hätten und wenn ja, ob sie es in Inhalt oder Umfang modifiziert hätten. Die abgelehnten Firmen sollten auf die Frage antworten, ob sie das beim FFF eingereichte Forschungsprojekt trotz Ablehnung weiter verfolgt haben. In Tabelle 40 sind die Ergebnisse zusammengefasst.

Tabelle 40: Additionalität des Programms [\%]

\begin{tabular}{lrr}
\hline & Teilnehmer & abgelehnte Bewerber \\
\hline wäre durchgeführt worden & 59,7 & 60,0 \\
wäre nicht durchgeführt worden & 32,5 & 40,0 \\
keine Angaben & 7,8 & 0,0 \\
\hline
\end{tabular}

Quelle: Fragebogenerhebung

Ca. 60\% der Teilnehmer gaben an, das Projekt in irgendeiner Form auch ohne die Unterstützung durchgeführt haben zu wollen. Dies ist auf den ersten Blick ein recht hoher Anteil, der sich allerdings relativiert, wenn man sich ansieht, in welcher Form das Projekt ohne Subvention durchgeführt worden wäre. Hier haben nur vier Unternehmen angegeben, dass sie das Projekt ganz ohne Änderungen abgewickelt hätten. Bei den weitaus meisten wäre es wohl zu Modifikationen sowohl im Projektumfang als auch in der Projektdauer gekommen. Von den fünf abgelehnten Firmen haben immerhin vier das Projekt trotzdem durchgeführt, wenn auch mit Änderungen in den Projektinhalten (Tabelle 41).

Tabelle 41: Additionalität des Programms [\%]

\begin{tabular}{lrr}
\hline & Teilnehmer & abgelehnte Bewerber \\
\hline durchgeführt, mit verändertem Projektvolumen & 35 & 0 \\
durchgeführt, mit Änderungen von Projektinhalten & 20 & 50 \\
durchgeführt, mit einer veränderten Projektlaufzeit & 18 & 25 \\
durchgeführt, mit einer modifizierten Zielsetzung & 16 & 25 \\
durchgeführt, mit weniger Projektpartner & 6 & 0 \\
durchgeführt, jedoch ohne jede Änderungen & 5 & 0 \\
\hline
\end{tabular}

Quelle: Fragebogenerhebung

Die Ergebnisse liegen im internationalen Vergleich im guten Rahmen. Bei eher grund lagenorientierten Forschungsprogrammen, wie dem von Technopolis evaluierten Mikrosystemtechnik Förderprogramm in Deutschland, ist die Additionalität mit über $60 \%$ deutlich höher. Bei anwendungsorientierten Programmen sind die Werte allerdings vergleichbar bis niedriger. In einer von Kemp (2002) zitierten FraunhoferISI Evaluierung eines deutschen Förderprogramms in der Umwelttechnologie auf haben beispielsweise 36\% der Teilnehmer angegeben, dass sie das Projekt ohne Unterstützung nicht durchgeführt. Dieser Wert ist fast identisch mit den 35\% der Sonderaktion Holzforschung. Ebenfalls hätten 5\% der Teilnehmer das Projekt ohne Unterstützung ohne jegliche Änderungen durchgeführt. Diese 5\% sind die eigentlichen Trittbrettfahrer, und auch hier deckt sich der Wert mit dem der Sonderaktion Holzforschung. In der Fraunhofer Studie werden die 5\% als ein ausgesprochen gutes Ergebnis bewertet und wir haben keinen Grund dies nicht auch für die Sonderaktion Holzforschung zu tun. Vielleicht sogar in einem verstärkten Maße als bei dem deutschen Programm, da dort die Förderquote mit durchschnittlich 51\% deutlich höher war. 
Die Ergebnisse der Fragebogenauswertung decken sich mit den Eindrücken aus den Interviews. Hier haben eigentlich alle Gesprächspartner gesagt, dass sie das Projekt in einem kleineren Umfang bzw. nicht so schnell durchgeführt hätten. Die Teilnahme an der Sonderaktion Holzforschung hat hier nicht wirklich neue Projekte ins rollen gebracht, sondern durch die Unterstützung konnte das F\&E-Projekt viel intensiver und rascher bearbeitet werden. Hier spielt nicht zuletzt auch die Projektbegleitung des FFF eine Rolle, der zu Recht darauf achtet, dass Termine und Kosten eingehalten werden und die Projektdokumentation stimmt. Dies wurde als konstruktiver Druck empfunden, der die Projektarbeit professionalisiert hat.

\section{2 Ökonometrische Analyse}

Es gibt mittlerweile eine relativ umfangreiche wissenschaftliche Literatur, die sich mit den Auswirkungen von F\&E-Förderungen beschäftigt. Grundsätzlich wird anhand von Firmen- oder Branchendaten untersucht, ob eine F\&E-Förderung die Firmen zu mehr Forschung anspornt oder es lediglich zu Mitnahmeeffekten führt. Mitnahmeeffekte sind dann sehr wahrscheinlich, wenn es sich um sehr marktnahe Projekte handelt, die mit wenig Risiko verbunden sind und ein hohes Ertragspotenzial haben. Tabelle 42 gibt einen Überblick über einige relevante Arbeiten und deren Ergebnisse. Da jeweils andere Branchen, Zeiträume und Förderregimes untersucht wurden, ist der Vergleich auf einer Metaebene natürlich schwierig und ersetzt nicht den genauen Blick auf den konkreten Einzelfall.

Tabelle 42: Einfluss von F\&E-Subventionen-Studien auf Firmenebene

\begin{tabular}{llll}
\hline US Daten & & Europäische Daten & \\
\hline Autoren & Ergebnis & Autoren & Ergebnis \\
\hline Hamberg (1966) & Komplementär & Holemans, Sleuwagen (1988) & Komplementär \\
Shrives (1978) & Substitutiv & Antonelli (1989) & Komplementär \\
Carmichael (1981) & Substitutiv & Busom (2000) & Komplementär \\
Higgings, Link (1981) & Substitutiv & Toivanen, Niinen (1998) & Substitutiv \\
Link (1982) & Komplementär & Meeusen, Janssens (2001) & Komplementär \\
Lichtenberg (1984) & Substitutiv & Almus, Czarnitzky (2002) & Komplementär \\
Lichtenberg (1987) & Insignifikant & Czarnitzky, Fier (2002) & Komplementär \\
Lichtenberg (1988) & Substitutiv & & \\
Wallsten (2000) & Substitutiv & & \\
\hline
\end{tabular}

Quelle: David, Hall, Toole (2000), eigene Recherche

Eine Auffälligkeit ist aber evident: Während die meisten amerikanischen Studien eine crowding out diagnostizieren, ist die Situation in Europa umgekehrt. Entweder sind amerikanische Unternehmen geschickter beim Einwerben von Fördermitteln, oder europäische Förderinstitutionen vorausschauender bei der Vergabe von Fördermitteln. Oder es trifft das zu, was seit gut einem Jahrzehnt behauptet wird, dass es nämlich Innovationssysteme gibt, also spezifische Zuschnitte von Institutionen, Beziehungen, Wertesysteme etc..

\section{Modell}

Um zu überprüfen, ob die FFF Förderungen bei den Teilnehmern substitutive oder komplementäre Effekte ausgelöst haben, wird in Anlehnung an Irwin und Klenow (1996) oder Meeusen und Janssens (2001) folgendes Modell geschätzt: 
(3) $F E U M S_{i t}=\alpha_{i}+\sum_{k=1}^{K} \lambda_{k} Z_{k i t}+\beta_{1} F E U M S_{i t-1}+\beta_{2} F \ddot{O U M S} S_{i t}+\beta_{3} F \ddot{O U} U S_{i t-1}+e_{i t}$,

$i$ ist der Firmenindex und $t$ der Zeitindex. $e$ ist der Fehlerterm. Die abhängige Variable FEUMS ist die Forschungsintensität, d.h. Forschungsausgaben im Verhältnis zum Umsatz. Auf der rechten Seite der Gleichung gibt es ein Konstante $\alpha$, sowie als erklärende Variablen die F\&E-Intensität des Vorjahres, die FFF Unterstützung im Verhältnis zum Umsatz FÖUMS in der gegenwärtigen und in der Vorperiode, sowie einen Vektor $Z_{k}$ mit $K$ Kontrollvariablen Wir benutzen hier den Logarithmus des Umsatzes $\left(L O G U M S_{t}\right)$, die Wachstumsrate des Umsatzes $\left(U M S W A_{t}\right)$ sowie die kumulierte Anzahl von geförderten Projekten (PRO). Außerdem werden folgende Dummyvariablen verwendet: $A G$ zeigt an, ob es sich bei der Firma um eine Aktiengesellschaft handelt; $N B$ nimmt den Wert Eins an, wenn sich die Firma nicht um eine Förderung beworben hat; $M H$ zeigt an, wenn die Firma im Minderheitsbesitz ist.

Die Parameter $\beta_{2}$ und $\beta_{3}$ in (3) geben jeweils den kurzfristigen Effekt der Subventionen auf die eigenen F\&E-Ausgaben an. Der langfristige Bruttoeffekt der Förderung, der sich durch Hinzuziehung der abhängigen 'lagged' Variable ergibt, lautet:

(4) $\beta_{L}=\left(\beta_{2}+\beta_{3}\right) / \beta_{1}$

Der Nettoeffekt der Förderung lautet dementsprechend:

(5) $\beta *_{L}=\beta_{L^{-}} 1$

(5) gibt an, um wie viel sich die eigenen Forschungsausgaben der Firmen aufgrund einer Förderung ändern. Ist $\beta^{*}{ }_{L}$ signifikant größer Null, so ist dies ein Indiz für Additionalität, bei einem Wert unter Null liegt ein Substitutionseffekt vor.

\section{Schätzmethode}

Gleichung (3) enthält als erklärende Variable die F\&E-Intensität der Vorperiode, d.h. die Variable ist stochastisch in dem Sinne, als ihre Ausprägung von dem Störterm der Vorperiode abhängt. Der OLS Schätzer ${ }^{17}$ führt in einer solchen Situation zu verzerrten Ergebnissen, weshalb hier mit einem von Arellano und Bond (1991) entwickelten GMM (Generalized Methods of Moments) Schätzer gearbeitet wird. Er wird auch als 'dynamic panel data estimator' bezeichnet.

Wir kontrollieren allerdings nicht nach einem potentiellen selection bias, da wir zuwenig Daten von solchen Firmen haben, die nicht an der Sonderaktion teilgenommen haben, in anderen Merkmalen jedoch mit den Teilnehmern ähnlich sind. Ein selection bias kann auftreten, wenn die Förderinstitution die Teilnehmer nicht zufällig auswählt, sondern beispielsweise nur solche Firmen fördert, die durch eine steigende Forschungsquote bereits auf sich aufmerksam gemacht haben. Unsere Ergebnisse wären in diesem Fall nach oben verzerrt, d.h. es würde der Förderung ein zu hohe Wirkung beigemessen, weshalb Kauko (1996) die Meinung vertritt, dass die meisten empirischen Studien bis dato den Einfluss von Förderungen systematisch

17 OLS = Ordinary Least Squares ('Methode der kleinsten Quadrate') 
überschätzt haben. Methodisch müsste man diesen selection bias durch ein Gleichungs system in den Griff bekommen, bei dem zusätzlich die Förderentscheidung der die Gelder vergebenen Institution modelliert wird (etwa durch ein Heckman Sample Selection Modell). Studien, die diese Verzerrung berücksichtigen, kommen daher tendenziell eher auf einen substitutiven denn auf einen komplementären Effekt der Förderung. Wallsten (2000) beispielsweise findet anhand eines dreistufigen Modells heraus, dass jeder Dollar F\&E-Förderung im Rahmen des amerikanischen 'Small Business Innovation Research' Programmes die eigenen F\&EAusgaben der Firmen um genau diesen Dollar vermindert, es mit anderen Worten zu einem totalen crowding out kommt.

\section{Ergebnisse und Diskussion}

Die Ergebnisse der Schätzung sind in Tabelle 43 wiedergegeben. Da die Dummyvariablen keinen signifikanten Effekt hatten, sind sie letztendlich nicht in die angegebene Gleichung aufgenommen worden. Die signifikanten Koeffizienten sind fettgedruckt, sie können in diesem Modell als Elastizitäten interpretiert werden.

\section{Tabelle 43: $\quad$ Regressionsergebnisse der GMM Schätzung}

\begin{tabular}{lrrr}
\hline & Koeffizient. & $\mathrm{z}$ & Signifikanz \\
\hline FEUMS $_{\mathrm{t}-1}$ & $\mathbf{- . 5 5 3}$ & -2.14 & 0.032 \\
FÖUMS $_{\mathrm{t}}$ & $\mathbf{- . 1 9 9}$ & -0.80 & 0.423 \\
FÖUMS $_{\mathrm{t}-1}$ & $\mathbf{. 9 5 6}$ & 2.01 & 0.045 \\
LOGUMS $_{\mathrm{t}}$ & $\mathbf{- 1 . 2 8 2}$ & -8.72 & 0.000 \\
UMSWA $_{\mathrm{t}}$ & .0085 & 1.47 & 0.141 \\
KONST. & $\mathbf{. 0 6 3 7}$ & 4.04 & 0.000 \\
\hline
\end{tabular}

Quelle: Fragebogenerhebung, eigene Berechnungen

Der für unsere Fragestellung relevante Parameter lautet

(6) $\beta^{*}{ }_{L}=-0,513$

Das bedeutet, dass eine Förderung von $1 €$ langfristig zu einer Reduktion der selbstfinanzierten F\&E-Investitionen von 51 Cent führt. Anders ausgedrückt: Die Subvention von $1 €$ führt lediglich zu einer Steigerung der Brutto F\&E-Ausgaben von 0,49 Cents. Offenbar liegt hier ein gewisses Maß an crowding out vor. Selbst wenn man den insignifikanten Koeffizienten $\beta_{2}$ nicht berücksichtigt, liegt $\beta{ }^{*}$ bei knapp -0,4. Dies ist zwar deutlich besser als beispielsweise bei Wallsten (2000), aber sicher nicht optimal, zumal der Koeffizient durch Vernachlässigung des sample selection bias eher zu hoch ausfallen dürfte, das tatsächliche Maß an crowding out also unterschätzt wird.

Eine Erklärung ist, dass die Förderquote von durchschnittlich 25\% doch relativ niedrig ist. Im Zusammenspiel mit der recht kurzen Laufzeit der Förderungen (ca. 1 Jahr) ist es sicherlich schwer, noch wesentlich höhere Nettoeffekte zu erzielen. Wie wir gesehen haben, sind die meisten Projekte bereits in kurzer Zeit abgeschlossen und die Projektergebnisse werden sehr schnell kommerzialisiert. Das alles spricht nicht dafür, dass es sich hier um besonders langfristige und daher risikoreiche Forschung handelte, weshalb gewisse Mitnahmeeffekte unvermeidlich sind. Vor diesem Hintergrund ist des $\mathrm{Maß}$ an crowding out wahrscheinlich noch im Rahmen und wäre bei anderen Branchen, die mehr Forschungs(förderungs)erfahrung haben, sicherlich höher. 
Dass sich die Ergebnisse der Regressionsanalyse von den Befunden aus der Fragebogenerhebung erwartungsgemäß unterscheiden, unterstreicht die Gefahr von strategischem Antwortverhalten bei Interviews und Befragungen. Verknüpft man die Ergebnisse aus der statistischen Analyse mit denen der Fragebogen und Interviews, welches Fazit ist dann zu ziehen? Obwohl es leider zu gewissen Mitnahmeeffekten gekommen ist, so kann sich trotzdem die Qualität und die Effizienz der Forschung in den Unternehmen durch die Teilnahme an der Sonderaktion verbessert haben. Durch die Erfahrungen und Kontakte des FFF, von denen die teilnehmenden Firmen im Zuge ihres Projektes wesentlich profitieren, mag dazu führen, dass $1 € \mathrm{~F} \& \mathrm{E}$ Investition in einem geförderten Betrieb höhere Erträge bringt als in einen nichtgeförderten. Diese Vermutung stützt sich auf die von uns durchgeführten Interviews. Unisono wurde gesagt, dass man deutlich von der Erfahrung des FFF hinsichtlich der Abwicklung von F\&E-Projekten profitiert hat und dadurch in der Lage war, dass Projekt schnell und zielstrebig abzuwickeln. Ein relativ hoher Effizienzgewinn, auch wenn er anhand unserer Daten nicht zu überprüfen ist, ist daher durchaus wahrscheinlich. Das soll den FFF aber nicht davon abhalten, in Zukunft noch mehr auf potenzielle Mitnahmeeffekte zu achten. Eine mögliche Handlungsempfehlung in diesem Zusammenhang wäre die stärkere Fokussierung auf vorwettbewerbliche, risikoreichere und längerfristige Projekte mit einer längeren Laufzeit und einer höheren Förderquote. 


\section{Volkswirtschaftliche und außenwirtschaftliche Wirkungen}

In diesem Kapitel wird der Blickwinkel von der Firmen- auf die auf Branchenebene gewechselt. Mit aggregierten Zahlen von Statistik Austria und WIFO wird erstens ein Bild der österreichischen Holzindustrie gezeichnet und zweitens versucht, die beobachteten Entwicklungen mit der Sonderaktion Holzforschung in Beziehung zu setzen. Robuste Zusammenhänge $\mathrm{zu}$ finden ist auf diesem höheren Aggregationsniveau allerdings deutlich schwieriger als auf Firmenebene.

\subsection{Fragestellung}

Die Sonderaktion Holzforschung förderte die Prozess- und Produktinnovation in der Holzwirtschaft (Be- und Verarbeitung von Holz, Möbelerzeugung). Die Aktion schloss auch die Zulieferer (Forstwirtschaft, chemische Industrie, Hersteller von Schlössern und Beschlägen, Softwarehäuser, Datenverarbeiter u. a.) und die Hersteller von Investitionsgütern (Öfen, Hebezeuge, Forstmaschinen, Werkzeugmaschinen u. a.) für die Holzwirtschaft ein. Erfolgreiche Forschungs- und Entwicklungstätigkeiten stärken die internationale Wettbewerbsfähigkeit der heimischen Produzenten. Dies sollte sich auf die Produktion, die Beschäftigung und die Außenhandelsbilanz der Holzwirtschaft auswirken.

Die Förderungen im Rahmen der Sonderaktion Holzforschung wurden vom FFF in den Jahren 1996 bis 1999 bewilligt. Die Erfolge der Förderungen können sich also frühestens ab 1998 in den Kennzahlen der Holzwirtschaft niederschlagen. Der vorliegende Beitrag stellt die Entwicklungen der letzten Jahre mit der Förderungsintensität in den einzelnen Bereichen der Holzwirtschaft in Beziehung. Es wird versucht, daraus Schlüsse über den gesamtwirtschaftlichen Erfolg der Förderungen zu ziehen.

\subsection{Struktur und Dynamik der Holzwirtschaft}

Die Holzwirtschaft umfasst sehr unterschiedliche Produktionen aus den Bereichen be- und verarbeitendes Gewerbe und Industrie. Das Gemeinsame dieser Gruppe ist der Werkstoff Holz, wobei in Teilbereichen (z. B. Möbelherstellung) in hohem Maße Kunststoffe und Metalle das Holz ersetzt haben. Die letzte Vollerhebung der Leistungs- und Strukturdaten der Nicht-landwirtschaftlichen Produktions- und Dienstleistungsbereiche führte das Österreichische Statistische Zentralamt (nunmehr Statistik Austria) 1995 durch. Die Ergebnisse werden auf der Basis einer Stichprobenerhebung jährlich fortgeschrieben. Bei der Interpretation der Ergebnisse ist $\mathrm{zu}$ berücksichtigen, dass in Bereichen mit wenigen Unternehmen die Fortschreibungen mit einem hohen Stichprobenfehler behaftet sein können. Dies trifft z. B. für die Plattenindustrie zu.

\subsubsection{Ausgangslage 1995}

1995 beschäftigte die Holzwirtschaft 80.487 Personen, das waren 12,4\% der Beschäftigten in der Sachgütererzeugung bzw. 2,4\% der Erwerbstätigen in Österreich insgesamt (Tabelle 47). Gemessen an den Beschäftigten war die Herstellung von Möbeln die weitaus wichtigste Gruppe innerhalb der Holzwirtschaft 
(51,1\% der hier Beschäftigten); ihr folgte die Herstellung von Konstruktionsteilen (Türen, Fenster, Schalungsplatten, Parkette, Fertighäuser, Hallen, Leimbinder u. a. Bautischlerarbeiten) mit einem Anteil von 27,1\%, die Sägewerke (Sägewerke, Hobelwerke, Holzimprägnierungswerke) mit 12,7\%, die Plattenwerke (Furnier-, Sperrholz-, Holzfaser- und Holzspanwerke) mit 4,5\%, die Herstellung von Kleinwaren (Griffe, Stiele, Formen, Leisten, Kleiderbügel, Haushaltsartikel und Küchengeräte, Figuren, Schmuck, Intarsien, Schatullen, Spulen, Kork-, Flecht- und Korbwaren) mit 3,5\% und die Herstellung von Verpackungsmitteln (Paletten, Fässer, Kisten u. a.) mit 1,0\%.

Die Holzwirtschaft ist vornehmlich kleingewerblich organisiert. Je Unternehmen waren 1995 durchschnittlich nur zwölf Personen beschäftigt (Sachgütererzeugung insgesamt: 26 Personen). Innerhalb der Holzwirtschaft ist die Größenstruktur der Unternehmen sehr unterschiedlich. Großunternehmen überwogen 1995 in der Plattenerzeugung (150 Beschäftigte). Die Herstellung von Kleinwaren beschäftigte durchschnittlich nur fünf Personen je Unternehmen. Der Personalaufwand je unselbständig Beschäftigten erreichte 1995 in der Holzwirtschaft $24.523 €$ (um 29\% weniger als im Durchschnitt der gesamten Sachgütererzeugung). Mit Ausnahme der Plattenwerke zählten alle Bereiche der Holzwirtschaft zu den Niedriglohnbranchen. Die Bruttowertschöpfung je Beschäftigten betrug $199532.224 €$ (um 31\% weniger als in der gesamten Sachgütererzeugung). Die Produktivität der Plattenwerke übertraf den Durchschnitt der Sachgütererzeugung um 13\%, jene der Möbelherstellung lag um 39\% darunter. Die Lohnquote war innerhalb der Holzwirtschaft recht unterschiedlich. In den kapitalintensiven Sägewerken betrug sie $55 \%$, in der arbeitsintensiven Möbelherstellung 76\%.

\subsubsection{Entwicklungen 1995 bis 2000}

Die Produktionsdynamik der heimischen Sachgüterproduktion hängt einerseits von der nationalen und internationalen Nachfrage nach ihren Produkten (Marktwachstum), andererseits von ihrer internationalen Wettbewerbsfähigkeit (Marktanteilsentwicklung) ab. Innerhalb der Holzwirtschaft verzeichneten im Zeitraum 1995 bis 2000 die Plattenwerke und Sägewerke die höchsten Produktionszuwächse (Tabelle 48). Das Wachstum der Plattenproduktion beschleunigte sich in den letzten Jahren (Abbildung 2). Auch die Herstellung von Verpackungsmitteln ist überdurchschnittlich gewachsen. Die konsumnahen Bereiche der Holzproduktion (Möbel $+4 \%$, Kleinwaren -17\%) und der Bereich Investitionsgüter (Konstruktionsteile $+13 \%$ ) blieben im Wachstum zurück. 


\section{Abbildung 2: Entwicklung des Produktionswertes in der Holzwirtschaft [1995 $=100]$}

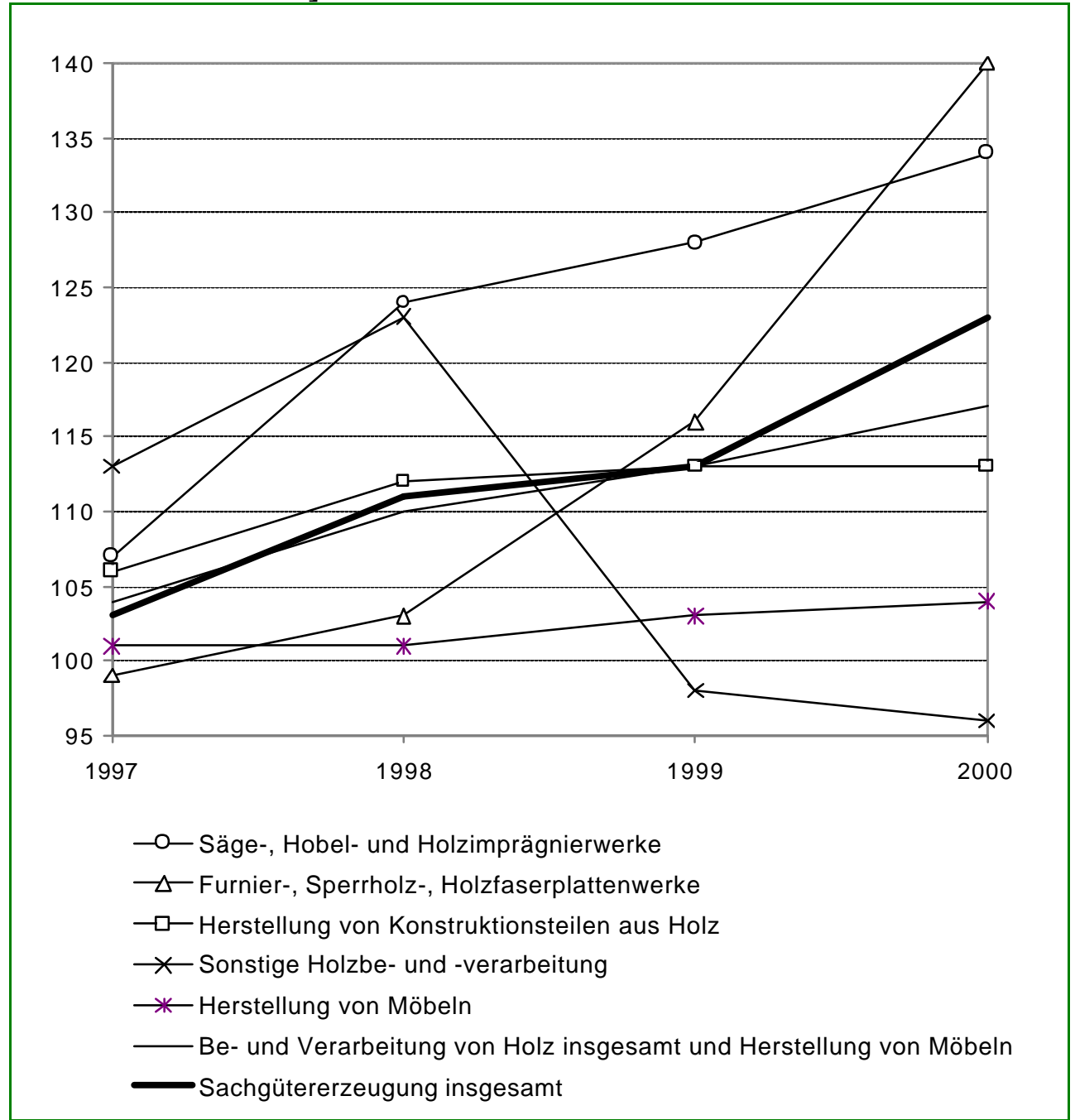

Quelle: Statistik Austria, eigene Berechnungen

Die Beschäftigung in der Holzwirtschaft nahm im Zeitraum 1995 bis 2000 stärker ab $(-6 \%)$ als in der gesamten Sachgütererzeugung $(-3 \%)$, wobei Plattenwerke $(+20 \%)$, die Hersteller von Verpackungsmitteln $(+9 \%)$ und die Sägewerke $(+5 \%)$ im Jahr 2000 mehr Personen beschäftigten als 1995. Die Zunahme der Beschäftigung der Plattenwerke im Jahr 2000 (Abbildung 3) dürfte auf einen Stichprobenfehler zurückzuführen sein. 
Abbildung 3: Entwicklung der Beschäftigten in der Holzwirtschaft [1995=100]

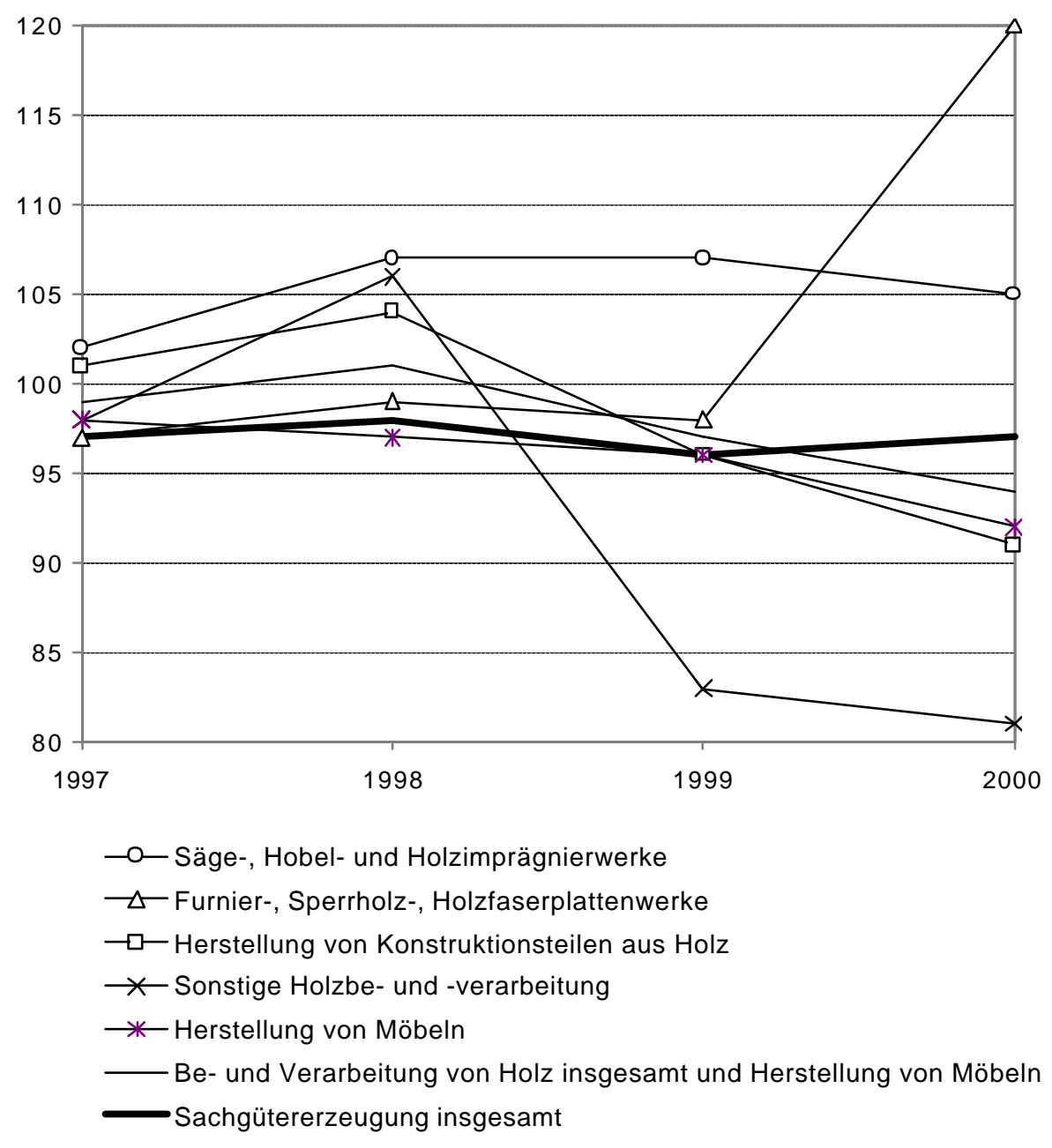

Quelle: Statistik Austria, eigene Berechnungen

Die Produktivitätsentwicklung (gemessen an der Wertschöpfung je Beschäftigten) der Holzverarbeitung hielt mit der Entwicklung in der gesamten Sachgüterproduktion mit $(+20 \%)$. Überdurchschnittlich stark stieg sie in den Sägewerken $(+31 \%)$, wobei sie sich in den letzten beiden Jahren besonders verbesserte (Abbildung 3). 
Abbildung 4: Entwicklung der We rtschöpfung je Beschäftigten in der Holzwirtschaft $[1995=100]$

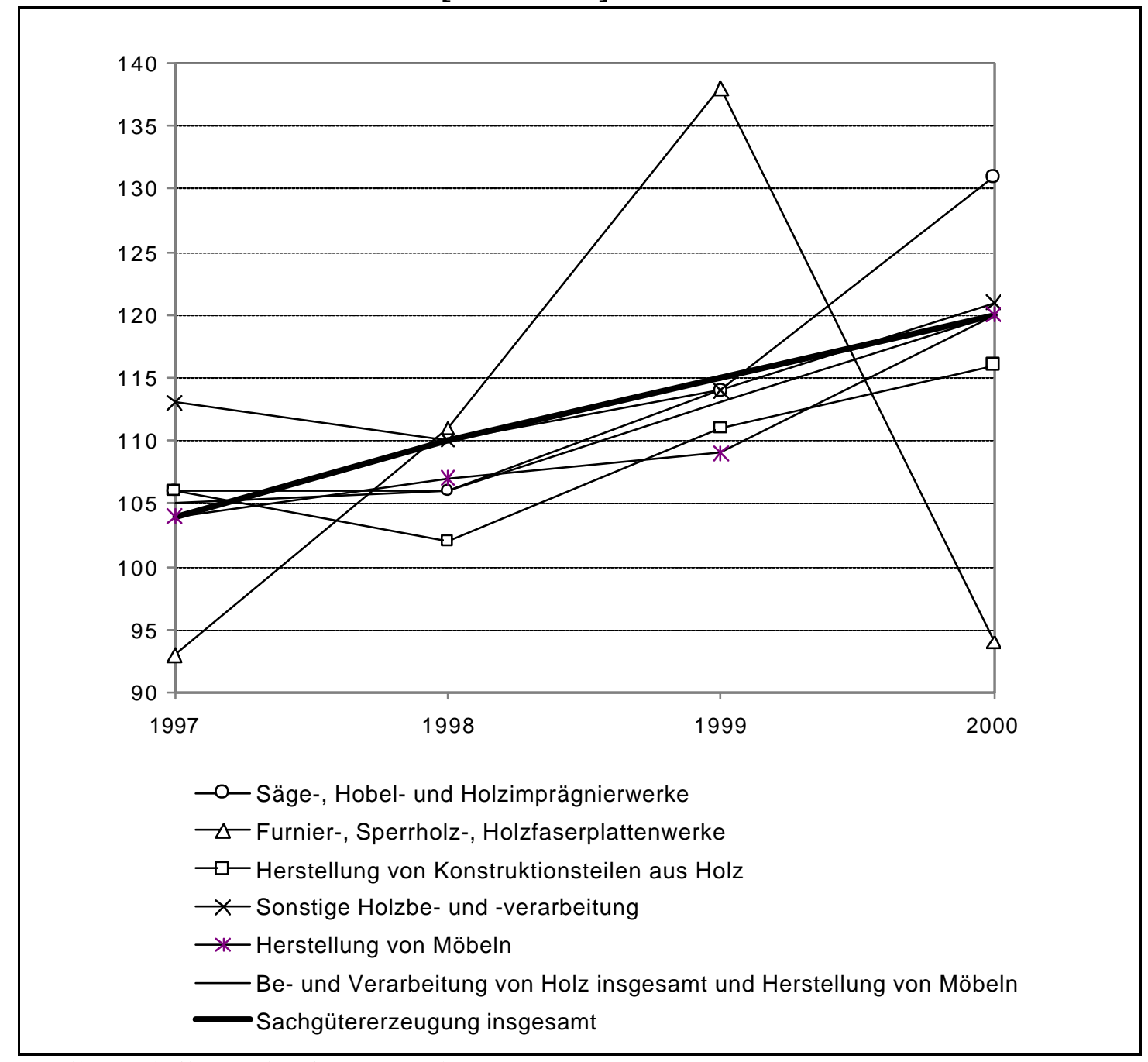

Quelle: Statistik Austria, eigene Berechnungen

Das Lohnniveau in der Holzwirtschaft näherte sich geringfügig dem durchschnittlichen Niveau der gesamten Sachgüterproduktion. Ab 1998 zogen die Löhne in den Sägewerken und in der Herstellung von Konstruktionsteilen stark an (Abbildung 5). 
Abbildung 5: Entwicklung des Personalaufwandes je unselbständig Beschäftigten in der Holzwirtschaft [1995 = 100]

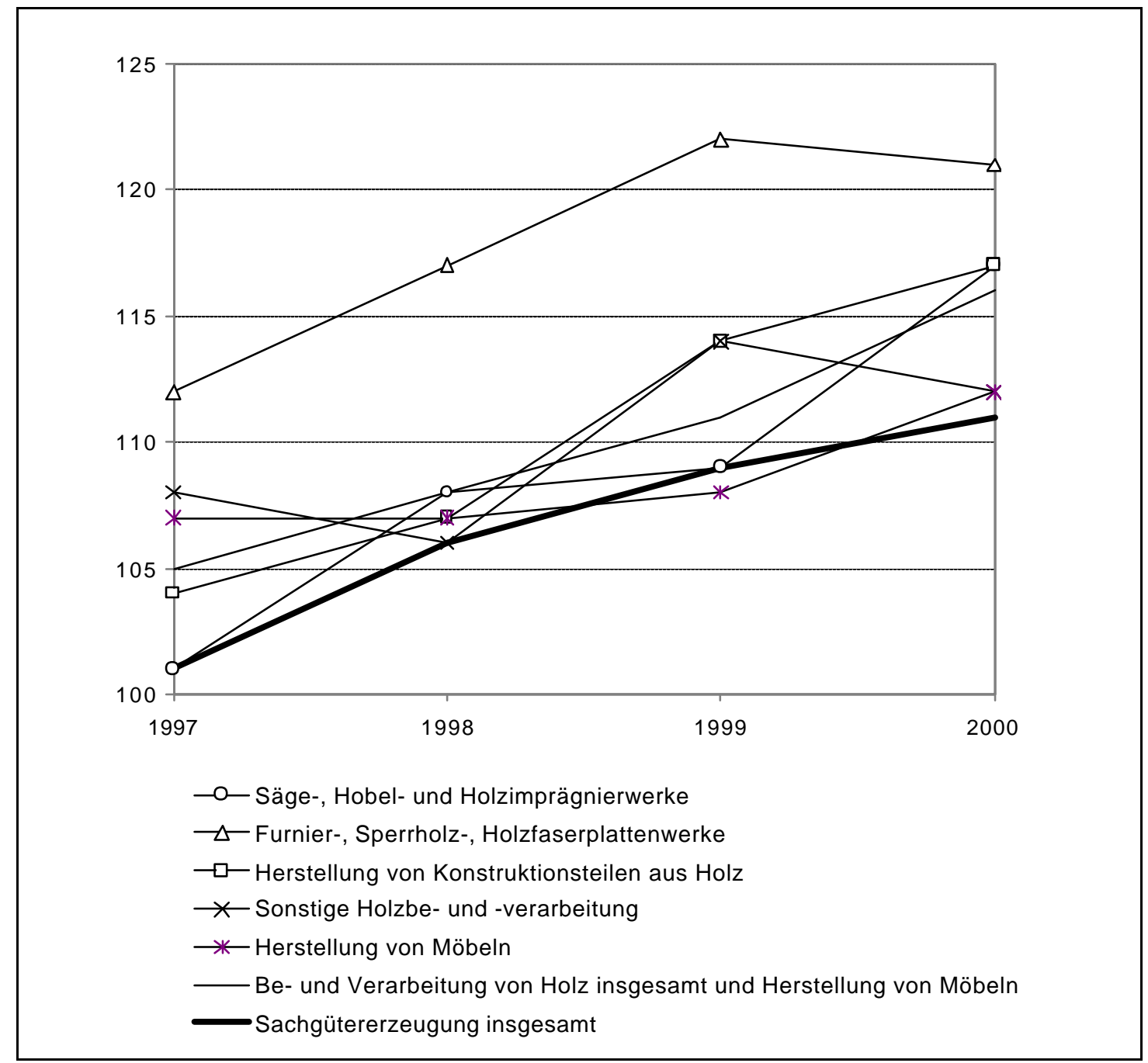

Quelle: Statistik Austria, eigene Berechnungen

\subsection{Forschungsförderung und Dynamik der Holzwirtschaft in den Jahren 1997 bis 2000}

Im Rahmen der Sonderaktion Holzforschung bewilligte der Forschungsförderungsfonds in den Jahren 1996 bis 1999 Förderungsmittel in der Höhe eines Barwertes von insgesamt 14,5 Mio. $€$. Davon entfielen rund $55 \%$ auf Projekte der Holzwirtschaft (Holzbe- und Verarbeitung, Möbelerzeugung). Der Rest verteilt sich auf die Forschung in Branchen, die mit der Holzwirtschaft verbunden sind (Forstwirtschaft, Maschinenbau, chemische Industrie). Den größten Anteil an der Forschungsförderung hatten mit $21 \%$ die Entwickler von Bearbeitungstechnologien für die Holzwirtschaft.

Es wurde geprüft, ob zwischen der Intensität der Forschung von Teilbereichen der Holzwirtschaft und ihrer Wachstumsdynamik in den Jahren 1998 bis 2000 ein Zusammenhang besteht. Eine Zuordnung der Förderung von Forschungsprojekten der Hersteller von Vorleistungen und Investitionsgütern für die Holzwirtschaft auf ihre Teilbereiche wurde dabei nicht vorgenommen. Es wurde also nur die 'direkte' 
Forschungsförderung der Holzwirtschaft untersucht; die Barwerte wurden entsprechend der NACE-Klassifizierung der Antragsteller auf die Teilbereiche der Holzwirtschaft aufgeteilt (Tabelle 49).

Gemessen an den Beschäftigten im Jahre 1995 waren die Plattenwerke der am stärksten (Barwert von $414 €$ je Beschäftigten, Tabelle 49), die Möbelherstellung (13 $€)$ der am wenigsten geförderte Bereich. Die Förderungsintensität, gemessen an der Wertschöpfung ergibt ein ähnliches Bild. Aufgrund der geringen Wertschöpfung je Beschäftigten liegt in dieser Kennzahlenreihung die Herstellung von Kleinwaren vor den Plattenwerken.

Die Forschungsförderung soll zur Entwicklung neuer und besserer Produkte und Produktionsverfahren führen. Eine erfolgreiche Umsetzung der Entwicklungen stärkt die Wettbewerbsstellung der geförderten Unternehmen: Sie wachsen rascher als ihre Konkurrenten, indem sie Marktanteile gewinnen aber auch zusätzliche Märkte für neue Produkte erschließen. Es wäre zu erwarten, dass in Summe die relative Höhe der Forschungsförderung mit dem Wachstum der Wertschöpfung korreliert. Verbesserungen im Produktionsablauf können Produktivitätssteigerungen bewirken, wodurch auch bei zunehmender Produktion die Beschäftigung sinkt. Dieser Zusammenhang kann 'gestört' werden:

- Auf stagnierenden oder schrumpfenden Absatzmärkten verschärft die Förderung hauptsächlich den Verdrängungswettbewerb, die gesamte Produktionsdynamik eines Bereiches ändert sich dadurch wenig.

- Das Wachstum in kürzeren Beobachtungszeiträumen (im konkreten Fall drei Jahre) wird stark durch konjunkturelle Schwankungen geprägt. Die Konjunkturzyklen der einzelnen Bereiche der Holzwirtschaft laufen nicht immer parallel.

- Die Ergebnisse der laufenden Produktions- und Beschäftigungsstatistik können mit Stichprobenfehler behaftet sein.

Aufgrund der relativ geringen Förderung (gemessen an den Beschäftigten oder der Wertschöpfung), des kurzen Beobachtungszeitraums (neue NACE-Gliederung ab 1995, Umsetzung von Forschungsergebnissen ab 1998) und des Stichprobenfehlers erscheint für die gegenständliche Evaluierung eine feinere Analyse (z. B. Bereinigung um Trend-Zykluseinflüsse) als wenig zielführend. Die Untersuchung beschränkt sich auf einen Vergleich der Förderungsintensitäten mit den Änderungen in den Wachstumsraten nach 1997. Auf mögliche konjunkturelle Einflüsse auf die Wachstumsgeschwindigkeiten wird hingewiesen.

Die Plattenwerke beschleunigten das Wachstum der Beschäftigung und der Wertschöpfung in der Periode 1997/2000 gegenüber der Periode 1995/1997 am stärksten. Sie konnten auch die Produktivität steigern (Tabelle 49). Die industrielle Plattenerzeugung betreibt an sich eine rege Forschungs- und Entwicklungstätigkeit. Dementsprechend stark beteiligte sie sich auch an der Sonderaktion Holzforschung. Recht günstig entwickelten sich auch die Sägewerke. Die Modernisierungs- und Rationalisierungsinvestition der letzten Jahre verbesserten die Produktivität maßgeblich. Die 'direkt' geförderte Forschungsintensität der Sägewerke lag unter dem Durchschnitt der gesamten Holzwirtschaft. Ihr kamen Innovationen im Bereich der maschinellen Ausrüstungen zugute. 
Die Produktion der Platten- und Sägewerke wurde durch die anhaltend gute Nachfrage im Zeitraum 1997/2000 begünstigt. Relativ kräftig gefördert wurde die Forschung in der Herstellung von Konstruktionsteilen und Kleinwaren. Sowohl die Beschäftigung als auch die Wertschöpfung dieser Bereiche verloren aber in den letzten drei Jahren stark an Dynamik. In der Produktivitätsentwicklung fielen sie zurück. Zum Teil ist die relativ ungünstige Entwicklung durch die schwache Baukonjunktur, zum Teil durch strukturelle Nachfrageänderungen erklärbar. Fenster und Kleinwaren aus Holz wurden durch Kunststoffprodukte verdrängt, der Importdruck aus Niedriglohnländern verstärkte sich.

Die Wertschöpfung in der Möbelherstellung nahm zu; bei leicht beschleunigtem Beschäftigungsabbau verbesserte sich die Produktivität seit 1997 relativ stark. Die 'direkte' Forschungsförderung war schwach. Eine Rangkorrelation zwischen Förderungsintensität (gemessen am Barwert je Beschäftigten oder in Prozent der Wertschöpfung) und der Wachstumsveränderung (in Prozentpunkten) zwischen dem Durchschnitt der Jahre 1995/1997 und 1997/2000 zeigte keinerlei Zusammenhang. Dies mag an der Inhomogenität der Produkte innerhalb der Bereiche der Holzwirtschaft liegen. So produziert der Bereich Konstruktionsteile für den stagnierenden Fenster- und Türenmarkt und für den expandierenden Markt für

Leimbinderelemente und Betonschalungssysteme. Eine Analyse der Außenhandelsdaten kann hier differenzierte Aufschlüsse über den Erfolg der Forschungsförderung bringen.

\subsection{Der Außenhandel mit Holz und Holzprodukten}

Eine gestärkte Wettbewerbsfähigkeit einer Branche schlägt sich in den Außenhandelsdaten nieder: Mit dem Gewinn von Marktanteilen im In- und Ausland verbessert sich die Handelsbilanz. Die exakte und detaillierte Erfassung des Außenhandels ermöglicht die Analyse einzelner Produkte der Holzwirtschaft.

\subsubsection{Außenhandelsverflechtung der heimischen Holzwirtschaft}

Die Außenhandelsverflechtung der Holzwirtschaft ist wesentlich geringer als in der übrigen Sachgüterproduktion: Im Jahr 2000 exportierte die Holzverarbeitung 49\% ihrer Produktion, die Sachgütererzeugung insgesamt 82\% (Tabelle 44). 
Tabelle 44: Außenwirtschaftskennzahlen [1995 und 2000]

\begin{tabular}{llrr}
\hline & & 1995 & 2000 \\
\hline Exportquote $^{1}$ & Sägeindustrie & 55,1 & 59,2 \\
& Holzverarbeitung & 31,0 & 43,8 \\
& Möbelerzeugung & 25,1 & 46,9 \\
& Holzwirtschaft insgesamt & 34,5 & 49,1 \\
& Sachgütererzeugung insgesamt & 62,8 & 81,8 \\
\hline Deckungsquote $^{2}$ & Sägeindustrie & 164,6 & 176,8 \\
& Holzverarbeitung & 110,5 & 122,4 \\
& Möbelerzeugung & 85,1 & 86,8 \\
& Holzwirtschaft insgesamt & 106,6 & 115,9 \\
& Sachgütererzeugung insgesamt & 94,5 & 98,4 \\
\hline Marktanteil Inland $^{3}$ & Sägeindustrie & 73,9 & 72,1 \\
& Holzverarbeitung & 76,2 & 68,8 \\
& Möbelerzeugung & 63,7 & 46,1 \\
& Holzwirtschaft insgesamt & 69,9 & 59,0 \\
& Sachgütererzeugung insgesamt & 35,1 & 17,9 \\
\hline
\end{tabular}

${ }^{1}$ Exportquote: Export/Produktionswert (Wert der technischen Produktion)

2 Deckungsquote: Produktionswert/Inlandsverbrauch (Produktionswert - Export + Import)

${ }^{3}$ Marktanteil Inland: (Produktionswert - Export)/Inlandsverbrauch.

Quelle: Statistik Austria, Konjunkturerhebung (WIFO-Datenbank)

Gleichzeitig betrug der Anteil der heimischen Holzwirtschaft am Inlandsverbrauch von Produkten der Holzwirtschaft 59\%, der Inlandmarktanteil der gesamten Sachgüterproduktion 18\%. Die traditionell geringe Außenhandelsverflechtung der Holzwirtschaft erklärt sich durch mehrere Faktoren. So überwiegt in der Produktion von Möbeln und Bauwaren noch das Kleingewerbe, das generell wenig geneigt ist, komplizierte Außenhandelsgeschäfte durchzuführen. Außerdem sind die Produkte sperrig, die Transportkosten hoch; eine intensive Beratung sowie Serviceleistungen sind notwendig; die länderspezifischen Unterschiede im Käufergeschmack aber auch in den Baunormen erschweren den internationalen Handel. Zur Zunahme der Außenhandelsintensität tragen Verbesserungen im Verpackungs-, Transport- und Normenwesen, die Ausweitung der industriellen und großgewerblichen Produktion bei.

Die Außenhandelsverflechtung der heimischen Wirtschaft nahm in den letzten fünf Jahren generell kräftig zu. Sie wurde durch den EU-Beitritt und die Ostöffnung wesentlich gefördert. Die Holzwirtschaft steigerte ihre Exportquote $(+42 \%)$ stärker als die Sachgütererzeugung insgesamt $(+30 \%)$. Innerhalb der Holzwirtschaft konnte die Möbelerzeugung die höchsten Zunahmen in der Exportquote erzielen. Die bereits stark exportorientierte Sägeindustrie legte nur mehr wenig zu. Der Grund dafür liegt in erster Linie im stagnierenden inländischen Rohholzangebot.

Die hohe Deckungsquote der Sägeindustrie erklärt sich primär durch den Waldreichtum Österreichs; aber auch die internationale Wettbewerbsstärke der heimischen Unternehmen trägt dazu bei. Die Plattenwerke haben eine ähnliche Stellung wie die Sägeindustrie. Die Möbelhersteller produzieren um rund 13\% weniger als der inländische Markt aufnehmen könnte.

Die heimische Holzwirtschaft verlor in den letzten fünf Jahren weniger Marktanteile im Inland als die Sachgütererzeugung insgesamt, wobei die Sägeindustrie ihre Marktanteile fast halten konnte und die Möbelindustrie stark verlor. 


\subsubsection{Die internationale Nachfrage nach Holz und Holzprodukten}

Für Holz und Holzprodukte mit ihren relativ niedrigen 'Unit Values' (Wert je Gewichtseinheit) sind die Transportkosten ein wesentlicher Wettbewerbsfaktor. Die Entfernung zu den wichtigen Importländern beeinflusst die Exportstruktur.

\section{Rohholz, einfach bearbeitetes Holz und Furniere}

Österreich ist eines der waldreichsten Länder in Europa, gleichzeitig aber auch der weitaus größte Importeur von Nadelrohholz (Tabelle 51). Dies deutet auf die Wettbewerbsfähigkeit der heimischen Säge-, Papier- und Plattenindustrie hin, die den Schweizer, süddeutschen und mittelosteuropäischen Waldbesitzern trotz der stärkeren Transportkostenbelastung die gleichen Preise bieten kann wie die dort ansässige Industrie.

Nadelschnitt- und Profilholz werden hauptsächlich in der Bauwirtschaft verwendet. Neben Großbritannien sind Italien und Deutschland die größten europäischen Importeure (Tabelle 51). Laubholz, hauptsächlich Tropenholz, daneben aber auch Buche und Eiche, wird am stärksten von Italien (Möbelindustrie) nachgefragt. Die österreichischen Exporteure von Schnitt- und Profilholz profitieren von ihrem Standort zwischen den beiden wichtigen Importländern Italien und Deutschland.

\section{Holzplatten}

Holzplatten (Spanplatten, Sperrholz, Faserplatten) werden vor allem in der Bau- und Möbelindustrie eingesetzt. Weitere Verbraucher sind die Autoindustrie, die Elektroindustrie u. ä. In Europa sind Deutschland und Großbritannien die größten Absatzmärkte für Holzplatten (Tabelle 52).

\section{Verpackungen und Bauwaren aus Holz}

Deutschland ist der wichtigste Importeur von Kisten und Paletten; Fässer haben in den Weinländern Italien, Frankreich und Spanien den stärksten Absatz (Tabelle 53). Die größten Importeure für Holzfenster, Holztüren und andere Bautischlerarbeiten in Europa sind Deutschland, Großbritannien, Frankreich und Italien.

\section{Fertighäuser, Möbel aus Holz.}

Deutschland importiert weltweit am meisten Fertighäuser. Weitere wichtige Importeure in Europa sind neben Frankreich auch die Schweiz und Österreich. Die starken europäischen Möbelimportländer Großbritannien und Frankreich aber auch die Niederlande (Küchenmöbel) (Tabelle 54) liegen transportmäßig für die österreichischen Möbelhersteller relativ ungünstig.

\subsection{3 Österreichs Exporte von Holzprodukten im Jahr 2001}

Die Exporte Österreichs von Holz und Holzprodukten (ohne Papier) erreichten im Jahr 2001 2,84 Mrd. €, das waren 4\% des Wertes aller Warenausfuhren. In den Exporten von Holz und Holzprodukten hatten Nadelschnittholz (35\%), Bautischlerarbeiten (ohne Holzfenster und Holztüren) (13\%), Spanplatten (12\%), Faserplatten (7\%), Wohnzimmermöbel (5\%) und Schaltafeln (4\%) die höchsten Anteile. 
Anhaltspunkte über die spezifischen (komparativen) Vorteile eines Landes für bestimmte Produktionen können aus den Außenhandelsbilanzen gewonnen werden. Einen ersten Überblick verschafft die Höhe des Exportüberschusses. Die höchsten Überschüsse in den Exporten der heimischen Holzwirtschaft ergaben sich 2001 für Schnittholz, Spanplatten, Bautischlerarbeiten (ohne Türen und Fenster), Faserplatten und Schaltafeln. Die Importüberschüsse erreichten für Nadelrundholz, Sitz- und Wohnzimmermöbel die größten Werte (Tabelle 50).

Bei internationalen Vergleichen von Export- oder Importüberschüssen ist zu berücksichtigen, dass die Höhe der Salden auch von der Landesgröße bzw. von der Stärke der außenwirtschaftlichen Verflechtung abhängt. Eine gebräuchliche Maßzahl für die internationale Wettbewerbsfähigkeit einer bestimmten Produktion, in die auch diese Faktoren eingehen, ist der RCA-Wert ('revealed comparative advantage'). Er ist der Logarithmus des Quotienten aus dem Export-Import-Verhältnis einer bestimmten Ware und dem Export-Import-Verhältnis einer entsprechenden Bezugswarengruppe. Ein RCA-Wert von Null zeigt an, dass für die betreffende Ware weder Standortvor- noch Nachteile realisiert wurden; positive RCA-Werte weisen auf bestehende und auch genutzte komparative Vorteile, negative auf Nachteile hin. Die in Tabelle 50 angeführten RCA-Werte sind auf den gesamten Außenhandel bezogen. In den RCA-Werten drücken sich somit die Standortvorteile aus, die die einzelnen Produktionen der Holzverarbeitung, verglichen mit der Gesamtproduktion, in Konkurrenz zur übrigen Welt haben.

Österreich ist nach diesem Konzept ein guter Standort für die Produktion von relativ rohholz- und transportintensiven Produkten wie Faserplatten, Spanplatten, Nadelschnittholz, Schaltafeln und Bautischlerarbeiten (ohne Holzfenster und Holztüren). Der negative RCA-Wert eines Waldlandes wie Österreich für Nadelrohholz erklärt sich aus der beschränkten inländischen Rohholzversorgung der im Export sehr erfolgreichen Säge-, Platten- und Papierindustrie. Die negativen RCA-Werte für Schlafzimmer-, Sitz- und Wohnzimmermöbel weisen auf hohe Erzeugungskosten, schwaches Design und Marketing am Produktionsstandort in Österreich hin.

\subsubsection{Dynamik der Exporte Österreichs}

Ein Erfolg der Sonderaktion Holzforschung sollte sich in der Dynamik der Exporte der geförderten Branchen auswirken, nämlich in zunehmendes Exportwachstum, und in der Verbesserung der Unit Values und RCA-Werte. Zu prüfen ist aber auch, ob die heimische Holzwirtschaft international Marktanteile gewann, d. h. ob ihre Exporte stärker wuchsen als jene der Konkurrenzländer.

\section{Holzprodukte, roh und einfach behandelt}

In dieser Gruppe erzielten ab 1998 die Exporte von Laubholz (Position anderes Holz, roh, gesägt, profiliert) die größten Zuwächse (Abbildung 6). Die 'Unit Values' wurden stark durch die internationale Holzpreisentwicklung geprägt (Abbildung 7). Die RCA-Werte der Laubholzsortimente verbesserten sich spürbar (Abbildung 8). Die Wettbewerbsstärke der Nadelprofilholzerzeugung ist, gemessen am RCA-Wert, gesunken. 
Trotz der wesentlichen Erleichterungen für Rohholzexporte in die EU (Abbau nichttarifärer Handelshemmnisse- insbesondere der Kontingentierung des grenzüberschreitenden Straßengüterverkehrs und Grenzaufenthalte) waren die österreichischen Nadelrohholzexporte nach 1997 rückläufig (Abbildung 18), ein Zeichen mehr für die Wettbewerbsstärke der heimischen Sägeindustrie. Die Laubholzexporte aus Österreich sind schwächer gestiegen als die aus Deutschland (Abbildung 19). Die Nadelschnittholzexporte nahmen zu, wogegen sie in Schweden und Finnland stagnierten (Abbildung 20). In den Exporten von Profilnadelholz trat nach dem starken Wachstum in den Jahren zuvor 1997 eine Abschwächung ein. Ähnliche Entwicklungen waren auch in Finnland und Schweden (Abbildung 21) zu beobachten. In den Laubschnitt- und Profilholzexporten profitierte sowohl Österreich als auch die europäische Konkurrenz von der starken Nachfragebelebung (Abbildung 22, Abbildung 23).

\section{Furniere, Platten aus Holz}

Die Exporte dieser Holzwaren nahmen in den letzten drei Jahren durchwegs kräftig zu (Abbildung 9). Besonders stark beschleunigte sich das Wachstum der Exporte von Faserplatten. Die 'Unit Values' von Sperrholz verschlechterten sich geringfügig, Span- und Faserplatten hielten das Niveau (Abbildung 10). Die RCA-Werte für Span- und Faserplatten verbesserten sich ab 1999 spürbar (Abbildung 11).

Österreichs Exporte von Furnieren aus Nadelholz waren von 1997 bis 2000 rückläufig, Marktanteile gingen verloren (Abbildung 24). Die Exporte von Laubholzfurnieren entwickelten sich hingegen sehr günstig (Abbildung 25). Am europaweiten Spanplattenmarkt konnten die österreichischen Exporteure ihre Marktstellung behaupten (Abbildung 26). Deutschland, Belgien und Frankreich verloren Marktanteile; Schweden, Polen und Italien erzielten starke Gewinne. Die Exporte von Sperrholz (mit Tropenholz oder veredelter Oberfläche) stiegen, ausgehend von einem niedrigen Niveau, kräftig (Abbildung 27). Das Wachstum der Exporte von Sperrholz aus Nadelholz endete 1997 (Abbildung 29), in den letzten drei Jahren waren sie rückläufig. Tschechien, Deutschland und Italien gewannen in Europa Marktanteile. Österreich ist nach Indonesien der wichtigste Exporteur von Schaltafeln (Abbildung 30). Trotz ungünstiger Baukonjunktur konnte ab 1997 wieder ein starkes Exportwachstum erzielt werden; Finnland, der Hauptkonkurrent in Europa, gewann Marktanteile.

Der Faserplattenmarkt expandierte weiter: Österreichs Industrie behauptete zwar seinen Rang in den Exporten von schweren Faserplatten (Abbildung 31), sie konnte aber mit dem Wachstum der Konkurrenz nicht mithalten. Im Bereich der mittelschweren (Abbildung 32) und leichten Faserplatten (Abbildung 33) erzielte Österreich in den letzten drei Jahren sehr gute Exporterfolge.

\section{Verpackungen, Bauwaren aus Holz}

Das Wachstum der Exporte von Bautischlerarbeiten (ohne Türen und Fenster) aus Österreich beschleunigte sich in den Jahren 1999 und 2000 (Abbildung 12). Die Exporte von Flachpaletten nahmen bereits ab 1997 stark zu. Wenig dynamisch entwickelten sich die Exporte von Holzfenster und Türen, hier mussten auch Preiseinbußen hingenommen werden (Abbildung 13). Die Preise für vorgefertigte 
Häuser zogen an. Die Wettbewerbsstellung, gemessen am RCA-Wert (Abbildung 14) verbesserte sich ab 1998 für Bautischlerarbeiten (ohne Fenster und Türen) und Flachpaletten.

In den Exporten für Kisten und Trommeln verlor Österreich ab 1997 deutlich Marktanteile an Deutschland, Tschechien und Schweden (Abbildung 34). Vom wachsenden Markt für Flachpaletten (Abbildung 35) profitierten Österreichs Exporteure. Kräftig wuchs auch der Markt für Fässer (Abbildung 36); Österreich konnte seine Stellung in Europa behaupten. Am Fenstermarkt teilte Österreich das Schicksal der deutschen, französischen und norwegischen Erzeuger; sie verloren Marktanteile an Polen, Großbritannien und Ungarn (Abbildung 37). Der Türenmarkt (Abbildung 38) entwickelte sich ab 1997 für alle europäischen Hersteller ungünstig.

Österreich ist in Europa der größte Exporteur von Konstruktionsholz (Abbildung 39). Das Exportwachstum beschleunigte sich in den letzten Jahren deutlich, wogegen die Konkurrenten in Deutschland und Schweden starke Einbußen hinnehmen mussten. Der Rückgang der Nachfrage nach Fertighäusern in Europa (Abbildung 40) wurde auch für die österreichischen Exporteure spürbar.

\section{Möbel}

In den Exporten für Büromöbel setzte ab 1996 ein kräftiges Wachstum ein (Abbildung 15). Die Exporte der Position andere Möbel aus Holz (hauptsächlich Wohnzimmermöbel) wuchsen nach einer kräftigen Zunahme 1995 in den folgenden Jahren stetig. Die Exporte von Küchen-, Schlafzimmer- und Sitzmöbeln nahmen ab 1999 stark ab. Aus der Entwicklung der Unit Values ist zu schließen, dass die Exporte von Küchen- und Schlafzimmermöbeln eher in das höhere Preissegment hineinwuchsen, wogegen der Unit Value der Exporte von Büro- und Wohnzimmermöbeln fiel (Abbildung 16). Gemessen am RCA-Wert konnte die Möbelindustrie lediglich die Wettbewerbsstellung im Bereich Wohnzimmermöbel leicht verbessern (Abbildung 17). Österreich verlor am kräftig wachsenden Weltmarkt für Möbel weitere Marktanteile. Dies betraf aber ebenso die starken Exportländer Italien und Deutschland. Kanada und China zählten zu den stärksten Gewinnern.

Im Export für Sitzmöbel (Abbildung 41) waren auch Mexiko, Malaysia, Thailand und Polen sehr erfolgreich. Im Büro- und Küchenmöbelbereich konnte Dänemark seine Position verbessern (Abbildung 42, Abbildung 43). Polen erreichte auch in den Schlafzimmerexporten eine starke Steigerung (Abbildung 44). Im Bereich Wohnzimmermöbel sind die italienischen Exporteure nach wie vor führend. China und Kanada konnten Dänemark vom zweiten Platz verdrängen (Abbildung 45).

\subsubsection{Veränderung der Handelsbilanz, der RCA-Werte und der Marktanteile 1997- 2000}

Von 1997 bis 2000 verbesserte sich der Außenhandelssaldo für Produkte der Holzwirtschaft um 301,5 Mio. $€$ (Tabelle 45). Besonders stark schlugen die absoluten Zuwächse in den Bereichen Nadelschnittholz, Bautischlerarbeiten (ohne Fenster und Türen), Faserplatten und Spanplatten zu Buche. Der Saldo für Nadelrohholz, Sitzmöbel, Küchenmöbel und Fertighäuser verschlechterte sich am stärksten. 
Tabelle 45: Veränderung der internationalen Wettbewerbsposition der österreichischen Holzwirtschaft [1997 - 2000]

\begin{tabular}{|c|c|c|c|c|c|c|c|}
\hline \multirow[t]{3}{*}{ SITC } & & $\begin{array}{l}\text { Export - } \\
\text { Import }\end{array}$ & RCA-Wert & \multicolumn{4}{|c|}{ Marktanteile } \\
\hline & & $\begin{array}{l}\text { Verände- } \\
\text { rung Saldo }\end{array}$ & $\begin{array}{l}\text { Veränder- } \\
\text { ung }\end{array}$ & & EU2000 & & Welt2000 \\
\hline & & In Mio. $€$ & & In $\%$ & $1997=100$ & In $\%$ & $1997=100$ \\
\hline 2474 & Nadelrohholz, unbehandelt & $-183,30$ & $-0,972$ & 2,7 & 52,6 & 0,7 & 60,5 \\
\hline 2475 & Anderes Rohholz, unbehandelt & $+3,91$ & $+0,489$ & 2,1 & 201,3 & 0,7 & 207,0 \\
\hline 2482 & Nadelholz ges. $>6 \mathrm{~mm}$ & $+222,57$ & $+0,025$ & 17,4 & 119,8 & 5,6 & 123,3 \\
\hline 2483 & Nadelholz profil. & $+2,91$ & $-0,173$ & 16,8 & 117,5 & 5,1 & 88,0 \\
\hline 2484 & Anderes Holz ges. $>6 \mathrm{~mm}$ & $-3,17$ & $+0,145$ & 0,9 & 103,7 & 0,7 & 134,3 \\
\hline 2485 & Anderes Holz profil. & $+4,52$ & $+0,219$ & 2,5 & 126,1 & 1,6 & 141,5 \\
\hline 63411 & Furniere aus Nadelholz & $+0,55$ & $+0,054$ & 2,0 & 89,3 & 1,0 & 71,3 \\
\hline 63412 & Furniere aus and. Holz & $+10,48$ & $+0,296$ & 1,8 & 112,3 & 1,9 & 137,7 \\
\hline 63422 & Span- u.ä. Platten aus Holz & $+47,09$ & $-0,063$ & 17,1 & 109,9 & 7,9 & 86,9 \\
\hline 63431 & Sperrholz mit Tropenholz & $-0,23$ & $+0,277$ & 0,7 & 129,5 & 0,2 & 169,8 \\
\hline 63439 & Anderes Sperrholz od. Furnier & $+2,24$ & $+1,585$ & 0,4 & 680,1 & 0,2 & 475,4 \\
\hline 63441 & Anderes Sperrholz ( Nadelholz) & $-2,71$ & $-0,168$ & 8,3 & 90,1 & 2,4 & 84,8 \\
\hline 63449 & $\begin{array}{l}\text { Anderes Sperrholz u.ä. a.n.g. } \\
\text { (Schaltafel) }\end{array}$ & $+20,22$ & $+0,196$ & 32,7 & 111,9 & 13,7 & 105,9 \\
\hline 63451 & Faserplatten $>0,8 \mathrm{~g} / \mathrm{cm} 3$ & $+49,69$ & $+0,192$ & 9,6 & 87,4 & 8,4 & 104,8 \\
\hline 63452 & Faserplatten $0,5-0,8 \mathrm{~g} / \mathrm{cm} 3$ & $+11,85$ & $+0,740$ & 2,8 & 86,3 & 1,4 & 88,0 \\
\hline 63453 & Faserplatten $0,35-0,5 \mathrm{~g} / \mathrm{cm} 3$ & $+0,61$ & $+1,531$ & 0,3 & 456,8 & 1,8 & 908,6 \\
\hline 63511 & Kisten, Trommeln u.ä. & $-1,60$ & $-0,231$ & 2,1 & 69,0 & 1,6 & 78,5 \\
\hline 63512 & Flachpaletten u.dgl. & $-5,70$ & $+0,145$ & 0,7 & 78,1 & 1,5 & 107,4 \\
\hline 6352 & Fässer, Tröge u.dgl. & $-1,67$ & $-0,596$ & 2,1 & 119,9 & 0,7 & 115,2 \\
\hline 63531 & Holzfenster u.dgl. & $+10,17$ & $+0,306$ & 4,7 & 104,4 & 3,2 & 94,7 \\
\hline 63532 & Holztüren u.dgl. & $+4,07$ & $+0,092$ & 2,5 & 82,2 & 1,5 & 75,3 \\
\hline 63539 & And. Bautischlerarbeit & $+150,02$ & $+0,399$ & 14,8 & 124,7 & 10,4 & 124,5 \\
\hline 811 & Vorgefertigte Häuser & $-16,14$ & $-0,227$ & 5,2 & 100,7 & 2,6 & 102,2 \\
\hline 82116 & Sitze a.n.g. mit Holzgestell & $-17,20$ & $-0,191$ & 1,4 & 62,8 & 0,7 & 60,9 \\
\hline 82151 & Büromöbel aus Holz & $-5,39$ & $-0,272$ & 3,8 & 108,7 & 1,9 & 73,7 \\
\hline 82153 & Küchenmöbel aus Holz & $-16,84$ & $-0,286$ & 6,3 & 72,8 & 3,1 & 66,1 \\
\hline 82155 & Schlafzimmermöbel aus Holz & $+17,65$ & $+0,045$ & 2,3 & 76,8 & 1,0 & 54,5 \\
\hline \multirow[t]{2}{*}{82159} & Andere Möbel aus Holz & $-3,10$ & $+0,005$ & 2,2 & 77,4 & 1,2 & 68,1 \\
\hline & Insgesamt & $+301,50$ & $+0,049$ & 5,4 & 98,5 & 2,9 & 103,5 \\
\hline
\end{tabular}

Quelle: Statistik Austria (WIFO-Datenbank), eigene Berechnungen

Gemessen an der RCA-Wertveränderung konnte die Holzwirtschaft ihre internationale Wettbewerbsstellung verbessern. Relativ stark waren die RCAWertzuwächse in den Bereichen leichte Faserplatten und Bautischlerarbeiten (ausgenommen Türen). Wesentliche Einbußen erlitten die Produzenten von Fässern, Küchen- und Büromöbeln.

Österreichs Holzwirtschaft steigerte ihren Anteil am Welthandel für Holzprodukte von 2,8\% auf 2,9\%. Der Anteil an den EU-Importen sank von 5,5\% auf 5,4\%. Die größten Marktanteilsverluste im EU-Handel ergaben sich für Nadelrohholz, Küchenmöbel und schweren Faserplatten. Die Marktanteilsgewinne waren für Schaltafel, Nadelschnittholz, Bautischlerarbeiten (ohne Fenster und Türen) sowie Nadelprofilholz besonders hoch. Bezogen auf den Welthandel wurden erhebliche Marktanteile für Bautischlerarbeiten (ohne Fenster und Türen), leichte Faserplatten, Nadelschnittholz und Schaltafel gewonnen, für Möbel, Spanplatten, Nadelprofilholz und Holztüren verloren.

\subsection{Forschungsförderung und internationale Wettbewerbsfähigkeit}

Eine erfolgreiche Forschungsförderung verbessert die internationale Wettbewerbsfähigkeit. In exportorientierten Branchen schlägt sich dies entsprechend stark in den Produktionszahlen nieder. Die Analysen im Abschnitt 2 zeigten kaum einen Zusammenhang zwischen der Beteiligung an der Sonderaktion Holzforschung und der Produktionsentwicklung der einzelnen Bereiche der Holzwirtschaft. Das erklärt 
sich zum Teil durch die weitgefächerten Produktpaletten. Die Außenhandelsstatistik erlaubt hier eine etwas detailliertere Analyse.

Die Forschungsintensität wurde an der Relation des Barwertes der Forschungsförderung zum Exportwert des Jahres 1997 gemessen. Im Durchschnitt der gesamten Branche erreichte der Barwert 3,9€ je $1.000 €$ Exportwert (Tabelle 46).

Tabelle 46: Forschungsförderung und Exporterfolge

\begin{tabular}{|c|c|c|c|c|c|c|c|c|}
\hline & \multirow{2}{*}{\multicolumn{2}{|c|}{$\begin{array}{l}\text { Barwert der } \\
\text { Förderung } \\
\text { Exportwert } 1997\end{array}$}} & \multicolumn{2}{|c|}{ Exportwachstum } & \multicolumn{2}{|l|}{ RCA-Werte } & \multicolumn{2}{|c|}{ RCA-Veränderung } \\
\hline & & & $1997-200$ & & 1997 & 2001 & $\begin{array}{l}1997- \\
2001\end{array}$ & \\
\hline & In $1.000 €$ & Rang & In \% p.a. & Rang & & & & Rang \\
\hline Schnittholz & 0,69 & 8 & $+6,4$ & 6 & $+1,409$ & $+1,378$ & $-0,031$ & 6 \\
\hline Platten usw. ${ }^{1}$ & 3,88 & 4 & $+11,3$ & 2 & $+0,995$ & $+1,206$ & $+0,211$ & 2 \\
\hline $\begin{array}{l}\text { Konstruktions- } \\
\text { teile }^{2}\end{array}$ & 12,05 & 2 & $+11,2$ & 3 & $+0,400$ & $+0,688$ & $+0,288$ & 1 \\
\hline Verpackungen & 1,77 & 5 & $+13,1$ & 1 & $-0,712$ & $-0,618$ & $+0,094$ & 4 \\
\hline Kleinwaren & 68,45 & 1 & $+10,1$ & 4 & $-0,737$ & $-0,585$ & $+0,152$ & 3 \\
\hline $\begin{array}{l}\text { Sitze a.n.g. mit } \\
\text { Holzgestell }\end{array}$ & 0,63 & 9 & $-1,1$ & 8 & $-0,792$ & $-0,956$ & $-0,164$ & 7 \\
\hline $\begin{array}{l}\text { Büromöbel aus } \\
\text { Holz }\end{array}$ & 6,84 & 3 & $+6,8$ & 5 & $+0,576$ & $+0,361$ & $-0,215$ & 8 \\
\hline $\begin{array}{l}\text { Küchenmöbel } \\
\text { aus Holz }\end{array}$ & 0,87 & 6 & $-4,4$ & 9 & $+0,296$ & $-0,088$ & $-0,384$ & 9 \\
\hline $\begin{array}{l}\text { Sonstige Möbel } \\
\text { aus Holz }\end{array}$ & 0,75 & 7 & $+1,0$ & 7 & $-0,783$ & $-0,754$ & $+0,029$ & 5 \\
\hline Insgesamt & 3,90 & & $+7,4$ & & $+0,463$ & $+0,600$ & $+0,137$ & \\
\hline
\end{tabular}

1 ohne Schaltafeln

2 einschließlich Schaltafeln und Fertighäuser

Quelle: FFF, Statistik Austria, eigene Berechnungen

Am stärksten wurde die Forschung in der Produktion von Kleinwaren und Konstruktionsteilen gefördert (gemessen an den Exporten). Die Exporte dieser Produktionen entwickelten sich nach 1997 besonders dynamisch, die RCA-Werte verbesserten sich überdurchschnittlich stark. Am wenigsten beteiligten sich die Sägewerke und Hersteller von Holzsitzmöbeln an der Forschungsförderung. Ihre Exportdynamik blieb zurück, die RCA-Werte verschlechterten sich. Die Rangkorrelation bestätigt den positiven Zusammenhang zwischen Förderungsintensität (gemessen am Barwert je $1.000 €$ Exportwert) und dem Exportwachstum (Rangkorrelation 0,60) sowie der Verbesserung des RCAWertes (Rangkorrelation 0,53) nach 1997. 


\section{Schlussfolgerungen}

Die Sonderaktion Holzforschung ist abgeschlossen. Es ist daher angebracht, zurück zu blicken, dabei aber nicht nur die spezifischen, auf Holzforschung gerichteten Beobachtungen zusammenzuführen, sondern auch über den Anlass hinaus zu blicken um für andere, ähnlich gelagerte Förderprogramme Lektionen zu erschließen. Dies soll in diesem abschließenden Kapitel geschehen.

\section{Innovativer Ansatz innerhalb des FFF}

Die Sonderaktion Holzforschung stellt einen innovativen Schritt innerhalb des FFF dar. Es handelt sich dabei (i) um ein the matisches Top-down-Programm, welches (ii) von allen relevanten Akteuren des Holzsektors entwickelt und getragen wurde. Dies ist allein aus historischer Perspektive - das Programm wurde in den Jahren 1994 und 1995 entwickelt - erwähnenswert. Diese Vernetzung relevanter politischer Akteure in der Programmentwicklung hat sich auch darin ausgedrückt, dass (iii) vorlaufend und parallel zum Forschungsförderprogramm eine Reihe von Innovationen im institutionellen bzw. regulatorischen Bereich (insb. Bauordnungen, Brandschutzbestimmungen) durchgeführt wurden. Hier wurde ansatzweise eine integrative Politik praktiziert. Dies ist wegen ihrer Seltenheit ausdrücklich zu würdigen und sollte als Richtschnur für künftige Programme gelten.

\section{Evaluierungskultur}

Eine zweite Würdigung betrifft die Tatsache, dass in diesem Programm sowohl eine Zwischen- als auch eine ex-post Evaluierung durchgeführt wurde und dass insbesondere die Zwischenevaluierung dazu genutzt wurde, um Kurskorrekturen und Präzisierungen für den weiteren Verlauf der Sonderaktion Holzforschung vorzunehmen.

\section{Clusteransatz}

Während das Programm zwar auf den Holzsektor ausgerichtet war, blieb es im Einzelnen insofern offen, als es sich nicht auf jene Sektoren beschränkt hat, innerhalb derer die Holzwirtschaft organisiert ist und von denen etwa der einschlägige Maschinenbau oder Teile der chemischen Industrie ausgeschlossen sind. Retrospektiv kann man hier - innerhalb des Forschungsprogramms - einen sortenreinen Clusteransatz beobachten, unabhängig davon, dass dies in keiner Phase der Entstehung bzw. Abwicklung des Sonderaktion Holzforschung explizit erwogen wurde.

\section{Zielgruppe der Sonderaktion Holzforschung}

Hier ergeben sich einige interessante, bisweilen überraschende Beobachtungen. Die geförderten Projekte weisen unterschiedliche Größen auf und liegen zwischen $€ 7.500$ und $€ 400.000$. Die $8 \%$ der größten Projekte lukrieren 25\% der Fördersumme. Überproportional viele Projekte stammen aus Oberösterreich, was deshalb erwähnenswert ist, als dort eine Clusterinitiative im Holzbereich durchgeführt wird, die sich mit der Laufzeit der Sonderaktion Holzforschung zumindest überlappt. Die Förderhöhe liegt mit 27\% im Wesentlichen auf dem demselben Niveau wie andere FFF-Förderungen. Auffallend ist das hohe Ausmaß der Reduktion der anerkannten 
Kosten. Einem Projektvolumen von $€ 78$ Mio. stehen anerkannte $€ 57$ Mio. gegenüber, wobei vor allem bei den Personalkosten Reduktionen vorgenommen wurden. Hier lässt sich zweifellos durch gezielte Kommunikation der Unterschied in den Erwartungen verringern. Interessant ist auch, dass es keinen Zusammenhang zwischen Förderbarwert und Förderquote gibt. Die Förderquote hängt also nicht von der Projektgröße ab. Schließlich ist zu erwähnen, dass der Sektor, der am meisten gefördert wurde - Bearbeitungsmaschinen, 20,8\% der Fördermittel - nicht dem offiziellen Holzsektor angehört.

\section{Treffsicherheit der Förderkriterien}

Dies ist eines der herausragendsten und zugleich positivsten Ergebnisse. Zunächst ist zu erwähnen, dass alle Kriterien - ausge nommen der Faktor 'Umwelt' - signifikant mit Ablehnung bzw. Förderung korrelieren, am stärksten bei den markt- und umsetzungsbezogenen Kriterien. Dies eröffnet Potenziale für eine Vereinfachung des Kriteriensatzes. Am Erstaunlichsten ist aber die Beobachtung, dass die Kriterien eine hohe Prädiktorwirkung auf späteren Markterfolg haben, dabei vor allem die Einschätzung der Marktaus sichten. Dies lässt sich als hohe Kompetenz der Gutachter interpretieren.

\section{Charakterisierung der geförderten Firmen}

Generell sind die teilnehmenden Firmen größer, internationaler und innovations freudiger als die nicht-teilnehmenden. Sie haben nicht nur einen absolut höheren Umsatz, sondern sind auch deutlich schneller gewachsen als die Nicht-Teilnehmer. Die Teilnehmer sind nicht nur in den letzten Jahren stärker gewachsen als die Kontrollgruppe, sondern auch über einen längeren Zeitraum. Teilnehmende Firmen sind überdies jünger als Unternehmen aus der Kontrollgruppe. Fügt man diese Einzelbefunde zusammen, zeigt sich klar die langfristig größere Dynamik bei den teilnehmenden Firmen.

Eine im Muster analoge, wenngleich schwächere Entwicklung lässt sich in Bezug auf die Mitarbeiter beobachten. Seit 1996 haben die Teilnehmer ihre Mitarbeiterzahl um 36\% erhöht, die österreichische Holzindustrie insgesamt hat im selben Zeitraum dagegen $12 \%$ der Mitarbeiter abgebaut.

Teilnehmende Firmen sind nicht nur größer, sondern auch exportorientierter. Am meisten fällt aber auf, dass die nicht-teilnehmenden bevorzugt den regionalen Markt $(<100 \mathrm{~km})$ bedienen, während bei den Teilnehmern der nationale Markt $(>100 \mathrm{~km})$ dominiert.

Generell lässt sich hier ein aus anderen Förderaktionen bekanntes Phänomen beobachten, dass durch die Förderaktion eher die ohnehin schon wachstumsorientierten und innovationsfreudigen Unternehmen angesprochen und in ihren Bemühungen verstärkt werden.

\section{Teilnehmende Firmen sind professioneller 'aufgestellt'}

Bei den Teilnehmern gibt es wesentlich häufiger ein professionelles Management. Insgesamt korreliert die Firmengröße mit der Neigung zu einem externen Management. 
Nur 23\% der Nicht-Teilnehmer verfügen überhaupt über ein eigenes F\&E-Budget, entsprechend $0,3 \%$ Forschungsquote. Anders bei den Teilnehmern, wo fast zwei Drittel ein eigenes F\&E-Budget haben und im Schnitt 1,6\% (2001) des Umsatzes für F\&E ausgeben.

Die Teilnehmer beschäftigen deutlich mehr hochqualifiziertes Personal. 80\% der Teilnehmer beschäftigen mindestens einen Akademiker, bei der Kontrollgruppe hingegen ist dies nur rund die Hälfte. Dieser eine Ingenieur ist wesentlich für die Funktion eines Technological Gatekeepers und damit für den Zugang zu neuen Technologien und in der Folge für Innovation.

\section{Innovationsbarrieren halten sich generell in Grenzen}

Die drei wesentlichsten Barrieren sind (i) das zu hohe Risiko, (ii) Schwierigkeiten in der Finanzierung der Innovationen, (iii) zu kleine eigene Forschungskapazitäten. Andere Innova tions barrie ren spielen nur unmaßgebliche Rollen. Innovationen durchzuführen oder nicht ist offenbar weniger von den Barrieren bestimmt als von den Anreizen und der generellen 'Aufstellung'.

\section{Kooperation: Teilnehmende Firmen sind besser vernetzt}

Man erkennt aus den Analysen deutlich, dass die teilnehmenden Firmen besser vernetzt sind als die Unternehmen der Kontrollgruppe. Ungefähr jeder dritte Teilnehmer gab an, im Rahmen des Projektes mit mindestens einem Partner kooperiert zu haben. Diese Zusammenarbeit hat in deutlich mehr als der Hälfte der Fälle auch zu neuen Netzwerken geführt. Interessant ist, dass nur $8 \%$ der Kooperationspartner direkte Mitbewerber waren, die überwiegende Mehrzahl der Kooperationspartner waren hingegen Zulieferer und Kunden. Damit waren Probleme wie etwa die Offenlegung von Know-how allenfalls Probleme vom Hören-Sagen.

\section{Markt- / Wettbewerbsposition}

Hier zeigt sich deutlich, dass Teilnehmer eher weiter downstream in der Wertschöpfungskette angesiedelt sind. In der Holzbranche sind dies marketingintensive Produkte mit verhältnismäßig hoher Wertschöpfung. Dies legt die Vermutung nahe, dass die Teilnehmer es eher geschafft haben, ihre Produkte zu differenzieren und in der Lage sind, am Markt höhere Preise durchzusetzen. Dies wird auch dadurch erhärtet, dass bei den geförderten Firmen Produktinnovationen als Wettbewerbsvorteil an zweiter Stelle nach der Produktqualität und vor der Qualität des Personals rangiert.

Es zeigt sich aber auch, dass die meisten Unternehmen ihrer eigenen Einschätzung nach noch nicht gut genug für den Wettbewerb gerüstet sind. Besonders offensichtlich ist dieser Befund für die Teilnehmer bei den Kategorien 'Preis', 'Marketing' und 'Logistik'. Die Firmen scheinen sich so einzuschätzen, dass sie zwar das Geschäft von der Produktseite verstehen, es aber noch Verbesserungs potenziale bei den markt- und vertriebsrelevanten Kompetenzen gibt. Dieses Ungleichgewicht zwischen dem technologischen und dem marktseitigen Ende des Innovationsprozesses sollte jedenfalls mit besonderer Aufmerksamkeit wahrgenommen werden. Allerdings 
sollte diese Aufgabe eher an die einschlägigen Fachorganisationen und Interessenvertretungen als an den FFF adressiert werden.

\section{Bekanntheit des Programms und Kontaktaufnahme}

Hier gibt es einige sehr unmittelbare Beobachtungen. Der mit Abstand größte Teil der Teilnehmer ist direkt über den FFF zur Sonderaktion gekommen. Dies spricht dafür, dass der FFF in der Branche präsent ist und über eine hohe Reichweite verfügt. Gut bekannt sind übrigens auch andere Programme: (Holz)Cluster und (Holz)Kompetenzzentren. Es gibt also wenig Hinweise auf den vielfach behaupteten 'Förderdschungel'.

\section{Ziele und Zielerreichung}

Gut die Hälfte gab an, dass das geförderte Projekt nur eine von mehreren F\&EAktivitäten ist, für jeden 5. Teilnehmer (21,9\%) hingegen war es der Einstieg in die Holzforschung. Die Gruppe der Neueinsteiger ist deshalb wichtig, als davon ausgegangen werden kann, dass hier der Einfluss der FFF Förderung besonders hoch war.

Die drei wichtigsten Ziele waren 'Entwicklung neuer Produkte', 'Verbesserung der allgemeinen Wettbewerbsfähigkeit' sowie die 'Entwicklung neuer Prototypen'. Die mit der Teilnahme verbunden Ziele waren eher produktorientiert und vorwärtsgerichtet, während die defensiven Ziele wie die 'Reduktion der Mitarbeiter' eher unwichtig waren. Dieser Befund ist positiv zu beurteilen.

Interessant ist auch die Zeitspanne, in der die Ziele erreicht werden. Hier zeigt sich, dass (i) die meisten Ziele tatsächlich erreicht wurden, (ii) meist schon im ersten Jahr und (iii) dass wichtige Ziele schneller erreicht wurden als unwichtige Ziele - Relevanz fokus siert also offenbar Aufmerksamkeit. Schließlich ist (iv) zu erwähnen, dass das Ziel 'Reduktion der Mitarbeiterzahl' nicht nur keine Rolle gespielt hat, sondern im Gegenteil haben die teilnehmenden Firmen im Zuge der geförderten Projekte sogar 273 neue Mitarbeiter eingestellt. Hochgerechnet auf die Grund gesamtheit bedeutet das, das ca. 500 neue Arbeitsplätze rund um die Innovationsvorhaben entstanden sind.

\section{Schwierigkeiten im Projektverlauf}

Hier sind vor allem folgende Probleme aufgetreten: (i) Problemstellung war schwieriger als erwartet, (ii) Zeit und Kosten wurden unterschätzt, (iii) Projektmitarbeiter waren zu sehr mit dem Tages geschäft beschäftigt. Dies deutet darauf hin, dass viele Firmen Erfahrungsdefizite im F\&E-Bereich haben, wobei Einsteiger hiervon besonders betroffen sind. Während 93\% der Einsteiger die technische Problemstellung unterschätzt haben, betrug der Anteil bei den Versierten lediglich 73\%. Entsprechend gaben $81 \%$ der Einsteiger an, Zeit und Kosten falsch eingeschätzt zu haben, bei den Versierten waren dies nur 68\%. Die Schwierigkeiten konnten grosso modo in ca. 50\% der Fälle gelöst werden. Interessant ist, dass bei Clusterinitiativen, wo die Clustermanager ihre Rolle nicht zuletzt im Betreuen von Projekten sehen, der Anteil der ungelösten Probleme verschwindend gering ist. 


\section{Verwertung der Ergebnisse und Fortsetzung der F\&E-Tätigkeiten}

Für drei Viertel der Firmen, die bereits mit der Verwertung begonnen haben, vergingen nicht mehr als drei Jahre zwischen Projektbeginn und der Kommerzialisierung der Forschungs ergebnisse. Dies ist relativ schnell und es zeigt, dass die Sonderaktion Holzforschung von ihrer Anlage her - wie generell der FFF - sehr marktnah ausgerichtet war, was sich unmittelbar in den Förderkriterien und ihren jeweiligen Cewichtungen aus drückt. Gleichzeitig gibt es Aktivitäten mit langfristiger Perspektive. Im Besonderen zeigt sich, dass lediglich 15\% der Teilnehmer nach Projektablauf die F\&E-Aktivitäten in diesem Bereich wieder einstellen wollen. Der Rest möchte weitermachen und die Holzforschungsaktivitäten teilweise sogar noch verstärken, zur Hälfte allerdings nur mit neuen Fördermitteln.

\section{Kosten und Nutzen der Programmteilnahme}

Für 2/3 der Teilnehmer hat der Nutzen der Teilnahme an der Sonderaktion die damit verbundenen Kosten überwogen. Dies sieht auf dem ersten Blick nicht überragend aus, ist aber im Vergleich mit anderen Programmen eher hoch. Im Einzelnen ist die Einschätzung des Nettonutzens unabhängig von der F\&E-Erfahrung der Teilnehmer.

\section{Bewertung der administ rativen Programmabwicklung}

Hier bekommt der FFF von den Firmen durchwegs gute bis sehr gute Noten. Besonders zufrieden sind die Teilnehmer mit der Geschwindigkeit der Abwicklung und der Zugänglichkeit zu Informationen. Erwartungsgemäß haben die abgelehnten Bewerbern durchgängig eine schlechtere Meinung von den Leistungen des FFF.

\section{Additionalitäten}

Hier wurden mehrere Ansätze herangezogen, um die Additionalität der Sonderaktion Holzforschung zu beurteilen. Ein indirekter Indikator für Additionalität ist die oben angesprochene Fortsetzung der Forschungstätigkeit. Im Besonderen wurden aber folgende zwei Herangehensweisen gewählt. Die direkte Befragung der Teilnehmer und die Abschätzung mithilfe eines ökono metrischen Modells.

In direkter Befragung gaben ca. 60\% der Teilnehmer an, das Projekt in irgendeiner Form auch ohne die Unterstützung durchgeführt haben zu wollen, davon alle bis auf vier mit unterschiedlichen Modifikationen. Dieses Ergebnis liegt im internationalen Vergleich im guten Rahmen, zumal zu berücksichtigen ist, dass die geförderten Projekte durchwegs sehr marktnah waren.

Die ökonometrische Analyse zeigt ein etwas nüchterneres Bild. Im Besonderen kommt ein gewisser Crowding out Effekt zum Vorschein, wonach die Verfügbarkeit von Fördergeld eigenes, privates Geld substituiert. Im Falle der Sonderaktion Holzforschung bedeutet dies, dass eine Förderung von $1 €$ langfristig zu einer Reduktion der selbstfinanzierten F\&E-Investitionen von 51 Cent führt. Eine Erklärung ist, dass die Förderquote von durchschnittlich $25 \%$ doch relativ niedrig ist. Im Zusammenspiel mit der recht kurzen Laufzeit der Förderungen und der starken Marktorientierung ist es sicherlich schwer, noch wesentliche höhere Nettoeffekte zu erzielen. 
Verknüpft man die Ergebnisse aus der statistischen Analyse mit denen der Fragebogen und Interviews, welches Fazit ist dann zu ziehen? Obwohl es zu gewissen Mitnahmeeffekten gekommen ist, so gibt es trotzdem Hinweise, dass sich die Qualität und die Effizienz der Forschung in den Unternehmen durch die Teilnahme an der Sonderaktion verbessert haben. Durch die Erfahrung und die Kontakte des FFF, von denen die teilnehmenden Firmen im Zuge ihres Projektes wesentlich profitieren, mag dazu führen, dass $1 € \mathrm{~F} \& \mathrm{E}$-Investition in einem geförderten Betrieb am Ende höhere Erträge bringt als es durch den zusätzlichen EURO aufgrund der ökonometrischen Betrachtung nahegelegt wird. Diese Vermutung wird durch die geführten Interviews gestützt. Unisono wurde gesagt, dass man deutlich von der Erfahrung des FFF hinsichtlich der Abwicklung von F\&E-Projekten profitiert hat und dadurch in der Lage war, dass Projekt rasch und zielstrebig abzuwickeln. Ein relativ hoher Effizienzgewinn ist daher durchaus wahrscheinlich. Das soll den FFF aber nicht davon abhalten, in Zukunft noch mehr auf potenzielle Mitnahmeeffekte zu achten. Eine nicht unattraktive Perspektive wäre in diesem Zusammenhang die stärkere Fokussierung auf vorwettbewerbliche, risikoreichere und längerfristige Projekte mit längerer Laufzeit und höherer Förderquote.

\section{Forschungsförderung und Dynamik der Holzwirtschaft in den Jahren 1997 bis 2000}

Um den volkswirtschaftlichen Wirkungen der Sonderaktion Holzforschung zu analysieren, wurde geprüft, ob zwischen der Intensität der Forschung von Teilbereichen der Holzwirtschaft und ihrer Wachstumsdynamik in den Jahren 1998 bis 2000 ein Zusammenhang besteht. Eine Zuordnung der Förderung von Forschungsprojekten der Hersteller von Vorleistungen und Investitionsgütern für die Holzwirtschaft auf ihre Teilbereiche wurde dabei nicht vorgenommen. Es wurde also nur die 'direkte' Forschungsförderung der Holzwirtschaft untersucht; wobei die Barwerte entsprechend der NACE- Klassifizierung der Antragsteller auf die Teilbereiche der Holzwirtschaft aufgeteilt wurden:

Gemessen an den Beschäftigten im Jahre 1995 waren die Plattenwerke der am stärksten (Barwert von $414 €$ je Beschäftigten), die Möbelherstellung (13€ je Beschäftigten) der am wenigsten geförderte Bereich. Die Förderungsintensität, gemessen an der Wertschöpfung, ergibt ein ähnliches Bild. Aufgrund der geringen Wertschöpfung je Beschäftigten liegt in dieser Kennzahlenreihung die Herstellung von Kleinwaren vor den Plattenwerken. Die Forschungsförderung soll zur Entwicklung neuer und besserer Produkte und Produktionsverfahren führen. Eine erfolgreiche Umsetzung der Entwicklungen stärkt die Wettbewerbsposition der geförderten Unternehmen: Sie wachsen rascher als ihre Konkurrenten, indem sie Marktanteile gewinnen aber auch zusätzliche Märkte für neue Produkte erschließen. Es wäre zu erwarten, dass in Summe die relative Höhe der Forschungsförderung eines Bereiches der Holzwirtschaft mit ihren Wachstumskennzahlen (Wertschöpfung, Beschäftigung) korreliert.

Die Plattenwerke beschleunigten das Wachstum, sowohl der Beschäftigung als auch der Wertschöpfung, in der Periode 1997/2000 gegenüber der Periode 1995/1997 am stärksten. Sie konnten auch ihre Produktivitätsentwicklung verbessern. Die industrielle Plattenerzeugung betreibt an sich eine rege Forschungs- und Entwicklungs- 
tätigkeit. Dementsprechend stark beteiligte sie sich auch an der Sonderaktion Holzforschung.

Recht günstig entwickelten sich auch die Sägewerke. Die Modernisierungs- und Rationalisierungsinvestition der letzten Jahre verbesserten die Produktivität maßgeblich. Die 'direkt' geförderte Forschungstätigkeit der Sägewerke lag unter dem Durchschnitt der Holzwirtschaft. Ihr kommen Innovationen im Bereich der maschinellen Ausrüstungen zugute. Die Produktion der Platten- und Sägewerke wurde durch die anhaltend gute Nachfrage im Zeitraum 1997/2000 begünstigt.

Relativ kräftig gefördert wurde die Forschung der Herstellung von Konstruktionsteilen und der Kleinwaren. Sowohl die Beschäftigung als auch die Wertschöpfung verloren aber in den letzten drei Jahren stark an Dynamik. Auch in der Produktivitätsentwicklung fielen diese Bereiche zurück. Zum Teil ist die ungünstige Entwicklung auf die schwache Baukonjunktur, zum Teil auf strukturelle Nachfrageänderungen erklärbar. Holzfenster und Kleinwaren aus Holz werden durch Kunststoffprodukte sukzessive verdrängt.

Die Herstellung von Möbeln nahm trotz der geringen Forschungsförderung kräftig $\mathrm{zu}$.

Eine Rangkorrelation zwischen Förderungsintensität (gemessen am Barwert je Beschäftigten oder in Prozent der Wertschöpfung) und der Wachstumsveränderung zwischen dem Durchschnitt der Jahre 1995/1997 und 1997/2000 zeigte keinerlei Zusammenhang. Dies mag zum Teil auf die Inhomogenität der Produkte innerhalb der Bereiche der Holzwirtschaft liegen. So produziert der Bereich Konstruktionsteile für den stagnierenden Fenster- und Türenmarkt und für den expandierenden Markt für Leimbinderelemente und Betonschalungssysteme.

\section{Der Außenhandel mit Holz und Holzprodukten}

Die Verbesserung der Wettbewerbsfähigkeit einer Branche schlägt sich in den Außenhandelsdaten nieder: Mit dem Gewinn von Marktanteilen im In- und Ausland sinken die Importe und wachsen die Exporte. Die exakte und detaillierte Erfassung des Außenhandels ermöglicht die Analyse einzelner Produkte der Holzwirtschaft.

Die Exporte Österreichs von Holz und Holzprodukten erreichten im Jahr 2001 2,84 Mrd. €, das waren 4\% des Wertes aller Warenausfuhren. Innerhalb der Exporte von Holz und Holzprodukten hatten Nadelschnittholz (35\%), Bautischlerarbeiten (ohne Holzfenster und Holztüren) (13\%), Faserplatten (7\%), Wohnzimmermöbel (5\%) und Schaltafeln (4\%) die höchsten Anteile.

Anhaltspunkte über die spezifischen (komparativen) Vorteile eines Landes für bestimmte Produktionen können aus den Außenhandelsbilanzen gewonnen werden. Einen ersten Überblick verschafft die Höhe des Exportüberschusses. Die höchsten Überschüsse in den Exporten der heimischen Holzwirtschaft ergaben sich 2001 für Schnittholz, Spanplatten, Bautischlerarbeiten (ohne Türen und Fenster), Faserplatten und Schaltafeln. Die Importüberschüsse erreichten für Nadelrundholz, Sitz- und Wohnzimmermöbel die größten Werte. 
Bei internationalen Vergleichen von Export- oder Importüberschüssen ist zu berücksichtigen, dass die Höhe der Salden auch von der Landesgröße bzw. von der Stärke der außenwirtschaftlichen Verflechtung abhängt. Eine gebräuchliche Maßzahl für die internationale Wettbewerbsfähigkeit einer bestimmten Produktion, in die auch diese Faktoren eingehen, ist der RCA-Wert ('revealed comparative advantage'). Er ist der Logarithmus des Quotienten aus dem Export-Import-Verhältnis einer bestimmten Ware und dem Export-Import-Verhältnis einer entsprechenden Bezugswarengruppe. Ein RCA-Wert von Null zeigt an, dass für die betreffende Ware weder Standortvornoch nachteile realisiert wurden; positive RCA-Werte weisen auf bestehende und auch genutzte komparative Vorteile, negative auf Nachteile hin. Die in angeführten RCA-Werte sind auf den gesamten Außenhandel bezogen. In den RCA-Werten drücken sich somit die Standortvorteile aus, die die einzelnen Produktionen der Holzverarbeitung -verglichen mit der Gesamtproduktion- in Konkurrenz zur übrigen Welt haben.

Österreich ist nach diesem Konzept ein guter Standort für die Produktion von Faserplatten, Spanplatten, Nadelschnittholz, Schaltafeln und Bautischlerarbeiten (ohne Holzfenster und Holztüren). Relativ schlecht scheinen die Bedingungen für die Produktion von Nadelrohholz zu sein. Dieser Wert ergibt sich aus der beschränkten inländischen Rohholzversorgung der im Export sehr erfolgreichen Säge-, Plattenund Papierindustrie. Die negativen RCA-Werte weisen auf die Standortnachteile in der Erzeugung von Schlafzimmer-, Sitz- und Wohnzimmermöbel hin.

Ein Erfolg der Sonderaktion Holzforschung, also die Verbesserung der internationalen Wettbewerbsfähigkeit der Holzwirtschaft, sollte sich in den Veränderungen der RCA-Werte zwischen 1998 und 2001 zeigen.

Sehr stark verbesserte sich demnach die Wettbewerbsfähigkeit der heimischen Produzenten von:

- Laubschnitt- und - profilholz,

- Faserplatten und

- Bautischlerarbeiten (Leimbinder, Konstruktionsteile ausgenommen Türen und Fenster).

Verbesserungen gab es auch für:

- Spanplatten,

- Schaltafeln,

- Holztüren und Holzfenster.

Es verschlechterte sich die internationale Wettbewerbsfähigkeit der Hersteller von:

- Nadelprofilholz,

- Büro-, Küchen- und Wohnmöbel.

Da wir gesehen haben, dass die Plattenwerke der am stärksten, die Möbelherstellung der am wenigsten geförderte Bereich waren, zeigt diese Reihung der Entwicklung der Wettbewerbsfähigkeit zwischen 1998 und 2001 einen positiven Zusammenhang mit der Intensität der Forschungsförderung durch den FFF. 


\section{$9 \quad$ Literaturverzeichnis}

Aghion, P., Dewatripont, M., Rey, P. (1997): 'Corporate Governance, Competition Policy and Industrial Policy', European Economic Review, 41, 797-805.

Almus, M., Czarnitzky, D. (2002): 'The Effects of Public R\&D Subsidies on Firms' Innovation Activities: The Case of Eastern Germany', Journal of Business and Economic Statistics.

Antonelli, C. (1989): 'A failure-inducement model of resaerch and development expenditure', Journal of Economic Behaviour and Organization, 12, 159-180.

Arellano, M., Bond, S. (1991): 'Some Tests of Specification for Panel Data: Monte Carlo Evidence and an Application of Employment Equations', Review of Economic Studies, 58, 277-297.

Brandstetter, L., Sakihabara, M. (1998): 'Japanese research consortia: a microeconomic analysis of industrial policy', Journal of Industrial Economics, 46, 207-233.

Busom, I. (2000): 'An emprirical investigation of the effects of R\&D subsidies', Economics of Innovation and New Technology, 9(2), 111-148.

Carmichael, J. (1981): 'The effect of mission-oriented public R\&D spending on private industry', Journal of Finance, 36, 617-627.

Cohen, W. (1995): 'Emprirical Studies of Innovative Activities', in: Stoneman, P. (Hg.): 'Handbook of the Economics of Innovation and Technological Change', 182-264, Oxford.

Czarnitzki, D., Fier, A. (2002): 'Do Innovation Subsidies Crowd Out Private Investment? Evidence from the German Service Sector, Konjunkturpolitik', Applied Economics Quarterly, 48(1).

D’Aspremont, C., Jaquemin, A. (1988): 'Cooperative and Non-cooperative R\&D in Duopoly with Spillovers', American Economic Review, 78, 1133-1137.

David, P.A., Hall, B.H., Toole, A.A. (2000): 'Is public R\&D a complement or substitute for private R\&D? A review of the evidence', Research Policy, 29, 497-529.

Haarhoff, D., Narin, F., Scherer, F., Vopel, K. (1999): 'Citation Frequency and the Value of Patented Innovation', Review of Economics and Statistics, 81 (3), 511-515.

Hamberg, D. (1966): 'Essays on the Economics of Research and Development', New York: Random House. 
Higgins, R., Link, A. (1981): 'Federal support of technological growth in industry: some evidence of crowding out', IEEE Transactions on Engineering Management, 28, 86-88.

Holemans, B., Sleuwaegen, L. (1988): 'Innovation expenditure and the role of government in Belgium', Research Policy, 17, 375-379.

Hosmer, D., Lemeshow, S. (1989): 'Applied Logistic Regression', New York: Wiley.

Irwin, D., Klenow, P. (1996): 'High Tech R\&D subsidies- estimating the effects of SEMATECH', Journal of International Economics, 40, 323-344.

Kauko, K. (1996): 'Effectiveness of R\&D subsidies- a sceptical note', Research Policy, 25, 321-323.

Kemp, R. (2002): 'An integrated policy for innovation for the environment', in: P. Boekhold (Hg.): 'Innovation and Sustainable Development: Can Innovation Incentive Make a Difference?', IWT Observatory, 40, 37-54.

Klette T.J., Moen J., Griliches, Z. (2000): 'Do Subsidies to Commercial RD Reduces Market Failure? Microeconometric Evaluation Studies', Research Policy, 29, 471-495.

Kraft, K. (1989): 'Market Structure, Firm Characteristics and Innovative Activity', Journal of Industrial Economics, 37, 329-336.

Meeusen, W., Janssens, W. (2001): 'Substitution versus additionality: econometric evaluation by means of micro-economic data of the efficacy and efficiency of R\&D subsidies to firms in the Flemish Region', Univerisity of AntwerpCentre for the Economic Study of Innovation and Technology (CESIT), Discussion Paper, 01/2001.

Mueller, D.C., Tilton, J.E. (1969): 'Research and Development Costs as Barrier to Entry', Canadian Journal of Economics, 2, 570-579.

Nadiri, I. (1993): 'Innovations and Technological Spillovers', NBER Working Paper, 4423.

Ohler, Teischinger, Schörghofer (1998), 'Zwischenevaluierung der Sonerderaktion Holzforschung', mimeo.

Porter, M. E. (1990): 'The Competitive Advantage of Nations', London.

Reinganum, J. F. (1982): 'A dynamic game of R\&D: patent protection and competetive behaviour', Econometrica, 50, 671-688.

Schott, H. (2001): 'Export und Unternehmensgröße', Frankfurt: Peter Lang Verlag.

Schumpeter, J. (1942): 'Capitalism, Socialism, and Democracy', Cambridge: MIT Press. 
Scott, A., Steyn, G., Geuna, A., Brusconi, S., Steinmueller, E. (2001): 'The Economic Returns to Basic Research and the Benefits of University.Industry Relationships: A Literature Review and Update of Findings', Report for the Office of Science and Technology by SPRU-Science and Techhnology Polica Research, Brighton.

Shankerman, M. (1981): 'The Effects of Double Counting and Expensing on the Measured Rates of Return', Review of Economics and Statistics, 63, 454-458.

Shrives, R. (1978): 'Market structure and innovation. A new perspective', Journal of Industrial Economics, 26, 329-347.

Toivanen, O., Niininen, P. (1998): 'Investment, R\&D subsidies and credit constraints', MITWorking Paper, Depart. Of Economics.

Wallsten, S. (2000): 'The effects of government-industry R\&D programs on private R\&D: the case of the Small Business Innovation Research Program', RAND Journal of Economics, 31 (1), 82-100 


\section{Appendix: Tabellen und Abbildungen}

\section{Tabelle 47: Kennzahlen der Holzwirtschaft [1995]}

\begin{tabular}{|c|c|c|c|c|c|c|c|c|c|c|c|}
\hline \multicolumn{2}{|c|}{ NACE } & \multirow[t]{2}{*}{ Unternehmen } & \multicolumn{2}{|c|}{ Beschäftigte am 31.12.1995 } & \multirow{2}{*}{$\begin{array}{l}\text { Personal- } \\
\text { aufwand } \\
\text { Mio. } €\end{array}$} & \multirow{2}{*}{$\begin{array}{l}\text { Produktions- } \\
\text { wert } \\
\text { Mio. } €\end{array}$} & \multirow{2}{*}{$\begin{array}{l}\text { Bruttowertschöpfung } \\
\text { zu Faktorkosten } \\
\text { Mio. } €\end{array}$} & \multirow{2}{*}{$\begin{array}{l}\text { Lohnquote }^{1} \\
\text { In } \%\end{array}$} & \multirow{2}{*}{$\begin{array}{l}\text { Beschäftigte } \\
\text { Je Unternehmen }\end{array}$} & \multirow{2}{*}{$\begin{array}{l}\text { Personalaufwand } \\
\text { Je unselbständig } \\
\text { Beschäftigten (€) }\end{array}$} & \multirow{2}{*}{$\begin{array}{l}\text { Bruttowertschöpfung } \\
\text { Je Beschäftigten ( } €)\end{array}$} \\
\hline & & & Insgesamt & $\begin{array}{l}\text { davon } \\
\text { unselbständig }\end{array}$ & & & & & & & \\
\hline 201 & $\begin{array}{l}\text { Säge-, Hobel- und } \\
\text { Holzimprägnierwerke }\end{array}$ & 1.311 & 10.221 & 8.894 & 228 & 1.526 & 413 & 55,3 & 7,8 & 25.642 & 40.365 \\
\hline 202 & $\begin{array}{l}\text { Furnier-, Sperrholz-, } \\
\text { Holzfaserplattenwerke }\end{array}$ & 24 & 3.592 & 3.582 & 117 & 600 & 190 & 61,8 & 149,7 & 32.779 & 52.856 \\
\hline 203 & $\begin{array}{l}\text { Herstellung von } \\
\text { Konstruktionsteilen aus } \\
\text { Holz }\end{array}$ & 1.458 & 21.816 & 20.523 & 512 & 1.638 & 713 & 71,7 & 15,0 & 24.924 & 32.683 \\
\hline 204 & $\begin{array}{l}\text { Herstellung von } \\
\text { Verpackungsmitteln aus } \\
\text { Holz }\end{array}$ & 112 & 870 & 762 & 17 & 60 & 26 & 64,9 & 7,8 & 22.042 & 29.731 \\
\hline \multirow[t]{2}{*}{205} & $\begin{array}{l}\text { Herstellung von } \\
\text { Holzwaren a.n.g., } \\
\text { Flecht- und }\end{array}$ & & & & & & & & & & \\
\hline & $\begin{array}{l}\text { Korbwaren } \\
\text { („,Kleinwaren“) }\end{array}$ & 582 & 2.826 & 2.275 & 50 & 150 & 76 & 66,3 & 4,9 & 22.034 & 26.757 \\
\hline 20 & $\begin{array}{l}\text { Be- und Verarbeitung } \\
\text { von Holz insgesamt }\end{array}$ & 3.487 & 39.325 & 36.036 & 924 & 3.974 & 1.417 & 65,2 & 11,3 & 25.638 & 36.031 \\
\hline \multirow[t]{3}{*}{361} & Herstellung von Möbeln & 3.191 & 41.162 & 38.254 & 898 & 2.454 & 1.177 & 76,3 & 12,9 & 23.473 & 28.587 \\
\hline & $\begin{array}{l}\text { Be- u. Verarbeitung von } \\
\text { Holz insgesamt und } \\
\text { Herstellung von Möbeln }\end{array}$ & 6.678 & 80.487 & 74.290 & 1.822 & 6.428 & 2.594 & 70,2 & 12,1 & 24.523 & 32.224 \\
\hline & $\begin{array}{l}\text { Sachgütererzeugung } \\
\text { insgesamt }\end{array}$ & 25.509 & 650.932 & 629.747 & 21.602 & 79.976 & 30.576 & 70,7 & 25,5 & 34.303 & 46.972 \\
\hline
\end{tabular}

${ }^{1}$ Personalaufwand in \% der Bruttowertschöpfung zu Faktorkosten.

Quelle: Statistik Austria, eigene Berechnungen 
Tabelle 48: Entwicklung der Kennzahlen der Holzwirtschaft [1995/2000]

\begin{tabular}{|c|c|c|c|c|c|c|c|c|c|c|c|}
\hline \multicolumn{2}{|c|}{ NACE } & \multirow{2}{*}{$\begin{array}{l}\text { Unternehmen } \\
1995=100\end{array}$} & \multicolumn{2}{|c|}{ Beschäftigte am 31.12.2000 } & \multirow[t]{2}{*}{ Personalaufwand } & \multirow[t]{2}{*}{ Produktionswert } & \multirow{2}{*}{$\begin{array}{l}\text { Bruttowertschöpfung } \\
\text { zu Faktorkosten }\end{array}$} & \multirow[t]{2}{*}{ Lohnquote $^{1}$} & \multirow{2}{*}{$\begin{array}{l}\text { Beschäftigte } \\
\text { Je } \\
\text { Unternehmen } \\
\end{array}$} & \multirow{2}{*}{$\begin{array}{l}\text { Personalaufwand } \\
\text { Je unselbständig } \\
\text { Beschäftigten }\end{array}$} & \multirow{2}{*}{$\begin{array}{l}\text { Bruttowertschöpfung } \\
\text { Je Beschäftigten }\end{array}$} \\
\hline & & & Insgesamt & $\begin{array}{l}\text { davon } \\
\text { unselbständig }\end{array}$ & & & & & & & \\
\hline 201 & $\begin{array}{l}\text { Säge-, Hobel- und } \\
\text { Holzimprägnierwerke }\end{array}$ & 96,9 & 104,5 & 105,2 & 123,0 & 133,7 & 136,9 & 89,8 & 107,8 & 116,9 & 131,0 \\
\hline 202 & $\begin{array}{l}\text { Furnier-, Sperrholz-, } \\
\text { Holzfaserplattenwerke²) }\end{array}$ & 116,7 & 120,4 & 120,3 & 146,0 & 140,1 & 113,4 & 128,7 & 103,2 & 121,4 & 94,2 \\
\hline 203 & $\begin{array}{l}\text { Herstellung von } \\
\text { Konstruktionsteilen aus } \\
\text { Holz }\end{array}$ & 84,6 & 90,9 & 90,9 & 106,5 & 112,8 & 105,8 & 100,7 & 107,4 & 117,2 & 116,4 \\
\hline 204 & $\begin{array}{l}\text { Herstellung von } \\
\text { Verpackungsmitteln } \\
\text { aus Holz }\end{array}$ & 96,4 & 109,3 & 111,5 & 117,3 & 127,6 & 126,3 & 92,8 & 113,4 & 105,1 & 115,6 \\
\hline 205 & $\begin{array}{l}\text { Herstellung von } \\
\text { Holzwaren a.n.g., } \\
\text { Flecht- und Korbwaren } \\
\text { (,Kleinwaren“) }\end{array}$ & 68,4 & 71,7 & 72,6 & 83,7 & 83,5 & 87,2 & 96,0 & 104,8 & 115,4 & 121,7 \\
\hline 20 & $\begin{array}{l}\text { Be- und Verarbeitung } \\
\text { von Holz insgesamt }\end{array}$ & 87,2 & 96,1 & 96,7 & 114,6 & 124,1 & 115,3 & 99,4 & 110,3 & 118,5 & 119,9 \\
\hline \multirow[t]{3}{*}{361} & $\begin{array}{l}\text { Herstellung von } \\
\text { Möbeln }\end{array}$ & 106,4 & 92,4 & 91,6 & 102,7 & 104,3 & 110,9 & 92,7 & 86,8 & 112,2 & 120,0 \\
\hline & $\begin{array}{l}\text { Be- u. Verarbeitung } \\
\text { von Holz insgesamt } \\
\text { und Herstellung von } \\
\text { Möbeln }\end{array}$ & 96,3 & 94,2 & 94,0 & 108,7 & 116,5 & 113,3 & 96,0 & 97,8 & 115,6 & 120,2 \\
\hline & $\begin{array}{l}\text { Sachgütererzeugung } \\
\text { insgesamt }\end{array}$ & 98,2 & 96,6 & 96,7 & 107,2 & 123,2 & 116,3 & 92,2 & 98,4 & 110,8 & 120,4 \\
\hline
\end{tabular}

${ }^{1}$ Personalaufwand in \% der Bruttowertschöpfung zu Faktorkosten

2 Ergebnisse sind zum Teil wenig plausibel (hoher Stichprobenfehler)

Quelle: Statistik Austria, eigene Berechnungen 
Tabelle 49: Forschungsförderung und Wachstum in der Holzwirtschaft

\begin{tabular}{|c|c|c|c|c|c|c|c|c|c|c|c|c|c|c|c|}
\hline \multirow{4}{*}{ Differenz 97/00 - 95/97 } & \multicolumn{3}{|c|}{ Barwert der Förderung 1995} & \multicolumn{12}{|l|}{ Wachstum } \\
\hline & \multirow{3}{*}{$\begin{array}{r}\text { Je Beschäf- } \\
\text { tigten } \\
\text { in } €\end{array}$} & \multirow{2}{*}{\multicolumn{2}{|c|}{$\begin{array}{l}\text { Relation zur Brutto- } \\
\text { wertschöpfung }\end{array}$}} & \multirow{2}{*}{\multicolumn{3}{|c|}{$\begin{array}{l}\text { Beschäftigte } \\
\varnothing \text { jährliche Veränderung 1995/1997 } \\
1997 / 2000\end{array}$}} & \multirow{2}{*}{\multicolumn{4}{|c|}{$\begin{array}{l}\text { Bruttowertschöpfung } \\
\varnothing \text { jährliche Veränderung 1995/1997 } \\
1997 / 2000\end{array}$}} & \multirow{2}{*}{\multicolumn{4}{|c|}{$\begin{array}{l}\text { Produktivität } \\
\text { Øjährliche Veränderung 1995/1997 } \\
\text { 1997/2000 }\end{array}$}} & \multirow[b]{3}{*}{ Rang } \\
\hline & & & & & & & & & & & & & & & \\
\hline & & in $\%$ & Rang & in $\%$ & in $\%$ & $\begin{array}{r}\text { in \% } \\
\text { Punkten }\end{array}$ & Rang & in $\%$ & in $\%$ & $\begin{array}{r}\text { in } \% \\
\text { Punkten }\end{array}$ & Rang & in $\%$ & in $\%$ & $\begin{array}{r}\text { in \% } \\
\text { Punkten }\end{array}$ & \\
\hline $\begin{array}{l}\text { Säge-, Hobel- und } \\
\text { Holzimprägnierwerke }\end{array}$ & 60,3 & 0,1 & 4 & $+0,9$ & $+0,9$ & $-0,08$ & 3 & $+4,0$ & $+8,2$ & $+4,20$ & 2 & $+3,0$ & $+7,3$ & $+4,25$ & 1 \\
\hline $\begin{array}{l}\text { Furnier-, Sperrholz- und } \\
\text { Holzfaserplattenwerke }\end{array}$ & 414,0 & 0,8 & 2 & $-1,3$ & $+7,3$ & $+8,66$ & 1 & $-4,9$ & $+7,8$ & $+12,76$ & 1 & $-3,6$ & $+0,5$ & $+4,11$ & 2 \\
\hline $\begin{array}{l}\text { Herstellung von Konstruk- } \\
\text { tionsteilen aus Holz }\end{array}$ & 215,3 & 0,7 & 3 & $+0,5$ & $-3,4$ & $-3,90$ & 5 & $+3,3$ & $-0,3$ & $-3,56$ & 5 & $+2,8$ & $+3,3$ & $+0,47$ & 4 \\
\hline $\begin{array}{l}\text { Herstellung von } \\
\text { Verpackungsmitteln aus Holz }\end{array}$ & 29,4 & 0,1 & 5 & $-1,0$ & $+3,7$ & $+4,77$ & 2 & $+6,1$ & $+3,9$ & $-2,14$ & 4 & $+7,2$ & $+0,2$ & $-6,99$ & 6 \\
\hline $\begin{array}{l}\text { Herstellung von Holzwaren } \\
\text { a.n.g., Flecht- und Korbwaren }\end{array}$ & 243,4 & 0,9 & 1 & $-1,0$ & $-9,9$ & $-8,92$ & 6 & $+4,7$ & $-7,3$ & $-11,96$ & 6 & $+5,7$ & $+2,9$ & $-2,81$ & 5 \\
\hline Herstellung von Möbeln & 12,7 & 0,0 & 6 & $-1,0$ & $-2,0$ & $-0,95$ & 4 & $+1,0$ & $+2,8$ & $+1,76$ & 3 & $+2,1$ & $+4,8$ & $+2,78$ & 3 \\
\hline Zusammen & 99,9 & 0,3 & & $-0,4$ & $-1,7$ & $-1,35$ & & $+1,9$ & $+3,0$ & $+1,09$ & & $+2,2$ & $+4,8$ & $+2,52$ & \\
\hline
\end{tabular}

Quelle: FFF, Statistik Austria, eigene Berechnungen 
Tabelle 50: Österreichischer Außenhandel mit Holz und Holzprodukten [1998 und 2001]

\begin{tabular}{|c|c|c|c|c|c|c|c|c|}
\hline \multirow{2}{*}{ SITC } & \multicolumn{2}{|c|}{ Export (2001) } & \multicolumn{2}{|c|}{ Import (2001) } & \multicolumn{2}{|c|}{ Saldo (Mio. €) } & \multicolumn{2}{|c|}{ RCA-Wert ${ }^{1}$} \\
\hline & Mio. $€$ & $1998=100$ & Mio. $€$ & $1998=100$ & 1998 & 2001 & 1998 & 2001 \\
\hline 2474 Nadelrohholz, unbehandelt & 34,67 & 77,2 & 349,51 & 140,0 & $-204,71$ & $-314,84$ & $-1,632$ & $-2,253$ \\
\hline 2475 Anderes Rohholz, unbehandelt & 40,47 & 220,3 & 63,86 & 114,6 & $-37,33$ & $-23,39$ & $-1,026$ & $-0,398$ \\
\hline 2483 Nadelholz profil. & 60,77 & 89,1 & 25,58 & 136,4 & $+49,48$ & $+35,19$ & $+1,375$ & $+0,923$ \\
\hline 2484 Anderes Holz ges., > $6 \mathrm{~mm}$ & 64,85 & 155,3 & 84,04 & 115,5 & $-31,01$ & $-19,19$ & $-0,472$ & $-0,201$ \\
\hline 2485 Anderes Holz profil. & 25,51 & 141,6 & 22,35 & 109,9 & $-2,31$ & $+3,16$ & $-0,037$ & $+0,191$ \\
\hline 63411 Furniere aus Nadelholz & 4,99 & 115,8 & 4,52 & 93,2 & $-0,54$ & $+0,47$ & $-0,037$ & $+0,156$ \\
\hline 63412 Furniere aus and. Holz & 44,30 & 143,6 & 53,43 & 148,3 & $-5,18$ & $-9,13$ & $-0,072$ & $-0,129$ \\
\hline 63422 Span- u. ä. Platten aus Holz & 352,94 & 122,4 & 58,39 & 102,5 & $+231,32$ & $+294,55$ & $+1,705$ & $+1,857$ \\
\hline 63431 Sperrholz mit Tropenholz & 10,98 & 143,7 & 27,22 & 119,3 & $-15,18$ & $-16,24$ & $-1,011$ & $-0,850$ \\
\hline 63439 Anderes Sperrholz oder Furnier & 6,10 & 968,3 & 9,90 & 79,5 & $-11,83$ & $-3,80$ & $-2,907$ & $-0,427$ \\
\hline 63441 Anderes Sperrholz aus Nadelholz & 17,75 & 133,0 & 21,12 & 104,2 & $-6,91$ & $-3,37$ & $-0,334$ & $-0,115$ \\
\hline 63449 Anderes Sperrholz u. ä. a.n.g. (Schaltafel) & 107,25 & 131,5 & 22,04 & 113,0 & $+62,06$ & $+85,21$ & $+1,514$ & $+1,640$ \\
\hline 63451 Faserplatten, $>0,8 \mathrm{~g} / \mathrm{cm} 3$ & 151,11 & 179,8 & 48,58 & 118,0 & $+42,87$ & $+102,53$ & $+0,797$ & $+1,193$ \\
\hline 63452 Faserplatten, 0,5 bis $0,8 \mathrm{~g} / \mathrm{cm} 3$ & 30,34 & 158,7 & 12,10 & 103,0 & $+7,37$ & $+18,24$ & $+0,571$ & $+0,977$ \\
\hline 63511 Kisten, Trommeln u. ä. & 4,26 & 125,7 & 8,00 & 126,4 & $-2,94$ & $-3,74$ & $-0,541$ & $-0,571$ \\
\hline 63512 Flachpaletten udgl. & 19,38 & 155,4 & 38,48 & 129,2 & $-17,31$ & $-19,10$ & $-0,787$ & $-0,628$ \\
\hline 6352 Fässer, Tröge udgl. & 4,64 & 254,9 & 5,74 & 526,6 & $+0,73$ & $-1,10$ & $+0,593$ & $-0,155$ \\
\hline 63531 Holzfenster udgl. & 21,36 & 86,5 & 23,81 & 74,7 & $-7,18$ & $-2,45$ & $-0,172$ & $-0,050$ \\
\hline 63532 Holztüren udgl. & 23,86 & 103,0 & 32,87 & 87,1 & $-14,58$ & $-9,01$ & $-0,405$ & $-0,262$ \\
\hline 63539 a. Bautischlerarbeit & 362,09 & 159,5 & 154,48 & 114,5 & $+92,05$ & $+207,61$ & $+0,603$ & $+0,910$ \\
\hline 811 Vorgefertigte Häuser & 81,40 & 125,1 & 82,96 & 130,0 & $+1,23$ & $-1,56$ & $+0,102$ & $+0,039$ \\
\hline 82116 Sitze a.n.g. m. Holzgestell & 60,33 & 102,5 & 166,25 & 104,8 & $-99,80$ & $-105,92$ & $-0,908$ & $-0,956$ \\
\hline 82151 Büromöbel aus Holz & 48,70 & 121,9 & 35,99 & 141,9 & $+14,57$ & $+12,71$ & $+0,537$ & $+0,361$ \\
\hline 82153 Küchenmöbel aus Holz & 59,29 & 79,6 & 68,60 & 116,5 & $+15,55$ & $-9,31$ & $+0,318$ & $-0,088$ \\
\hline 82155 Schlafzimmermöbel aus Holz & 38,91 & 92,1 & 110,44 & 86,4 & $-85,54$ & $-71,53$ & $-1,024$ & $-0,985$ \\
\hline 82159 Andere Möbel aus Holz & 154,03 & 109,1 & 323,98 & 103,3 & $-172,30$ & $-169,95$ & $-0,714$ & $-0,685$ \\
\hline Insgesamt & $2.838,37$ & 126,5 & $2.028,87$ & 114,4 & $+470,18$ & $+809,50$ & $+0,319$ & $+0,394$ \\
\hline
\end{tabular}

${ }^{1}$ RCA: Revealed Comparative Advantage

Quelle: Statistik Austria (WIFO-Datenbank) 
Tabelle 51: Die wichtigsten Importeure von Rohholz, einfach bearbeitetem Holz und Furnieren [2000]

\begin{tabular}{|c|c|c|c|c|c|c|c|c|c|c|}
\hline & Länder & Japan & Südkorea & China & Österreich & Kanada & USA & Italien & Finnland & Deutschland \\
\hline & Importwert, in Mio. $€$ & $1.855,4$ & 448,3 & 409,8 & 397,6 & 229,8 & 204,8 & 178,2 & 163,9 & 152,6 \\
\hline & Unit Value, in $€$ je $\mathrm{kg}$ & 0,22 & 0,09 & 0,09 & 0,07 & . & 0,15 & 0,10 & 0,05 & 0,07 \\
\hline \multirow{3}{*}{$\begin{array}{l}\text { Anderes Rohholz, } \\
\text { unbehandelt }\end{array}$} & Länder & China & Japan & Italien & Frankreich & Spanien & Finnland & Kanada & Deutschland & Portugal \\
\hline & Importwert, in Mio. $€$ & $1.382,4$ & 667,7 & 361,4 & 262,0 & 227,0 & 215,7 & 190,8 & 167,4 & 162,7 \\
\hline & Unit Value, in $€$ je $\mathrm{kg}$ & 0,26 & 0,24 & 0,13 & 0,24 & 0,12 & 0,04 & 0,13 & 0,38 & 0,17 \\
\hline \multirow[t]{3}{*}{ Nadelholz ges. $>6 \mathrm{~mm}$} & Länder & USA & Japan & GB & Italien & Deutschland & Frankreich & Niederlande & Dänemark & Spanien \\
\hline & Importwert, in Mio. $€$ & $7.488,7$ & $2.806,9$ & $1.459,1$ & $1.021,9$ & 866,4 & 520,6 & 510,3 & 459,7 & 367,5 \\
\hline & Unit Value, in $€$ je $\mathrm{kg}$ & 0,27 & 0,53 & 0,37 & 0,29 & 0,30 & 0,35 & 0,29 & 0,37 & 0,33 \\
\hline \multirow[t]{3}{*}{ Nadelholz profil. u.ä. } & Länder & USA & Kanada & Japan & Deutschland & GB & Italien & Schweiz & Australien & Frankreich \\
\hline & Importwert, in Mio. $€$ & 677,9 & 91,7 & 90,2 & 70,7 & 57,8 & 38,2 & 31,9 & 27,5 & 25,9 \\
\hline & Unit Value, in $€$ je $\mathrm{kg}$ & . & . & 1,81 & 0,65 & 1,00 & 0,66 & 0,99 & 0,96 & 0,94 \\
\hline \multirow[t]{3}{*}{ Anderes Holz ges. $>6 \mathrm{~mm}$} & Länder & China & Italien & Japan & USA & Hongkong & Kanada & Spanien & Niederlande & GB \\
\hline & Importwert, in Mio. $€$ & 973,4 & 759,6 & 694,7 & 572,1 & 510,4 & 435,1 & 431,0 & 388,5 & 343,1 \\
\hline & Unit Value, in $€$ je kg & 0,45 & 0,49 & 0,87 & 0,49 & 0,62 & 0,53 & 0,56 & 0,74 & 0,75 \\
\hline \multirow[t]{2}{*}{ Anderes Holz profil. u.ä. } & Länder & USA & Japan & Italien & Kanada & Belgien & GB & Niederlande & Deutschland & Frankreich \\
\hline & Unit Value, in $€$ je $\mathrm{kg}$ & . & 1,98 & 1,43 & & 0,23 & 2,09 & 0,96 & 1,35 & 1,47 \\
\hline \multirow[t]{3}{*}{ Furniere aus Nadelholz } & Länder & USA & Japan & Frankreich & Spanien & Italien & Deutschland & GB & Kanada & Saudi-Arab. \\
\hline & Importwert, in Mio. $€$ & 121,8 & 29,0 & 24,6 & 23,7 & 21,0 & 18,4 & 15,7 & 14,3 & 13,2 \\
\hline & Unit Value, in $€$ je kg & 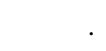 & 0,86 & 0,67 & 1,43 & 2,77 & 3,37 & 1,38 & & 0,81 \\
\hline \multirow[t]{3}{*}{ Furniere aus and. Holz } & Länder & USA & Deutschland & Italien & China & Kanada & Spanien & Japan & Südkorea & Frankreich \\
\hline & Importwert, in Mio. $€$ & 385,9 & 268,7 & 219,2 & 195,6 & 161,2 & 130,9 & 80,6 & 74,4 & 73,9 \\
\hline & Unit Value, in $€$ je kg & . & 2,70 & 1,55 & 0,42 & . & 2,11 & 1,12 & 0,64 & 1,90 \\
\hline
\end{tabular}

Quelle: UNO (WIFO-Datenbank) 
Tabelle 52: Die wichtigsten Importeure von Holzplatten [2000]

\begin{tabular}{|c|c|c|c|c|c|c|c|c|c|c|}
\hline \multirow[t]{3}{*}{ Span- u.ä. Platten aus Holz } & Länder & USA & Deutschland & Frankreich & Niederlande & GB & Japan & Kanada & Spanien & Italien \\
\hline & Importwert, in Mio. $€$ & $1.715,7$ & 307,4 & 177,6 & 171,1 & 157,0 & 134,6 & 114,2 & 104,9 & 103,6 \\
\hline & Unit Value, in $€$ je kg & 0,37 & 0,33 & 0,41 & . & 0,24 & 0,55 & . & 0,26 & 0,23 \\
\hline \multirow[t]{3}{*}{ Sperrholz mit Tropenholz } & Länder & Japan & USA & Deutschland & Südkorea & GB & Niederlande & Belgien & China & Mexiko \\
\hline & Importwert, in Mio. $€$ & $1.705,5$ & 853,6 & 287,5 & 274,2 & 236,0 & 169,8 & 147,8 & 136,2 & 123,9 \\
\hline & Unit Value, in $€$ je kg & . & & 0,85 & 0,49 & 0,70 & . & 0,77 & . & 0,27 \\
\hline \multirow[t]{3}{*}{ Anderes Sperrholz o. Furnier } & Länder & GB & Deutschland & China & USA & Japan & Niederlande & Mexiko & Italien & Frankreich \\
\hline & Importwert, in Mio. $€$ & 172,7 & 140,2 & 122,0 & 120,9 & 119,1 & 93,0 & 86,4 & 55,5 & 54,1 \\
\hline & Unit Value, in $€$ je kg & 0,63 & 0,61 & . & . & . & . & 0,16 & 0,66 & 0,80 \\
\hline \multirow{3}{*}{$\begin{array}{l}\text { Anderes Sperrholz aus } \\
\text { Nadelholz }\end{array}$} & Länder & Japan & USA & China & Deutschland & GB & Schweiz & Hongkong & Südkorea & Jordanien \\
\hline & Importwert, in Mio. $€$ & 150,2 & 123,2 & 60,0 & 52,5 & 37,8 & 36,9 & 29,8 & 22,4 & 22,0 \\
\hline & Unit Value, in $€$ je kg & & & & 0,92 & 0,83 & 2,70 & . & 1,20 & 0,51 \\
\hline Anderes Sperrholz u.ä. a.n.g. & Länder & China & Japan & Saudi-Arab. & Schweiz & USA & Deutschland & Italien & GB & Frankreich \\
\hline \multirow[t]{2}{*}{ (darunter Schaltafeln) } & Importwert, in Mio. $€$ & 154,7 & 75,2 & 68,9 & 66,8 & 65,6 & 34,3 & 26,7 & 20,6 & 20,1 \\
\hline & Unit Value, in $€$ je kg & & 1,35 & 0,69 & 1,14 & . & 0,99 & 0,90 & 1,32 & 1,01 \\
\hline \multirow[t]{3}{*}{ Faserplatten $>0,8 \mathrm{~g} / \mathrm{cm} 3$} & Länder & USA & GB & China & Frankreich & Belgien & Kanada & Deutschland & Italien & Polen \\
\hline & Importwert, in Mio. $€$ & 308,8 & 154,3 & 129,7 & 102,6 & 84,8 & 77,0 & 73,1 & 50,0 & 49,3 \\
\hline & Unit Value, in $€$ je kg & . & 0,67 & 0,59 & 0,63 & 0,45 & 0,92 & 0,41 & 0,32 & 0,65 \\
\hline \multirow[t]{3}{*}{ Faserplatten $0,5-0,8 \mathrm{~g} / \mathrm{cm} 3$} & Länder & USA & Japan & GB & China & Spanien & Deutschland & Belgien & Südkorea & Hongkong \\
\hline & Importwert, in Mio. $€$ & 254,9 & 146,2 & 102,0 & 100,5 & 72,8 & 71,2 & 70,2 & 65,7 & 58,8 \\
\hline & Unit Value, in $€$ je kg & . & 0,46 & 0,42 & 0,42 & 0,32 & 0,32 & 0,31 & 0,30 & . \\
\hline \multirow[t]{3}{*}{ Faserplatten $0,35-0,5 \mathrm{~g} / \mathrm{cm} 3$} & Länder & USA & Türkei & China & Mexiko & Kanada & Deutschland & Australien & GB & Spanien \\
\hline & Importwert, in Mio. $€$ & 23,4 & 7,4 & 6,2 & 4,9 & 4,2 & 3,2 & 3,1 & 1,6 & 1,5 \\
\hline & Unit Value, in $€$ je kg & & 0,26 & 0,25 & 0,21 & 0,79 & 0,27 & . & 0,57 & 0,31 \\
\hline
\end{tabular}

Quelle: UNO (WIFO-Datenbank) 
Tabelle 53: Die wichtigsten Importeure von Verpackungen und Bauwaren aus Holz [2000]

\begin{tabular}{|c|c|c|c|c|c|c|c|c|c|c|}
\hline \multirow[t]{3}{*}{ Kisten, Trommeln u.ä. } & Länder & USA & Deutschland & Frankreich & Belgien & Mexiko & Niederlande & Italien & Schweiz & Kanada \\
\hline & Importwert, in Mio. $€$ & 40,3 & 36,7 & 24,8 & 24,0 & 16,9 & 14,3 & 10,6 & 10,0 & 8,0 \\
\hline & Unit Value, in $€$ je kg & & 0,67 & 0,70 & 0,85 & 1,56 & 0,93 & 0,82 & 1,42 & \\
\hline \multirow[t]{3}{*}{ Flachpaletten u.dgl. } & Länder & Deutschland & USA & GB & Frankreich & Italien & Belgien & Niederlande & Spanien & Österreich \\
\hline & Importwert, in Mio. $€$ & 184,7 & 152,9 & 135,4 & 126,2 & 89,8 & 76,0 & 56,5 & 50,5 & 40,5 \\
\hline & Unit Value, in $€ \mathrm{je} \mathrm{kg}$ & 0,20 & . & 0,42 & 0,34 & 0,25 & 0,32 & . & 0,34 & 0,25 \\
\hline \multirow[t]{3}{*}{ Fässer, Tröge u.dgl. } & Länder & USA & Australien & Italien & GB & Frankreich & Spanien & Chile & Südafrika & Kanada \\
\hline & Importwert, in Mio. $€$ & 133,7 & 36,7 & 35,3 & 27,9 & 25,0 & 22,5 & 13,6 & 13,5 & 12,3 \\
\hline & Unit Value, in $€$ je kg & & . & 5,16 & 1,36 & 2,01 & 2,96 & . & 7,19 & \\
\hline \multirow[t]{3}{*}{ Holzfenster u.dgl. } & Länder & Deutschland & USA & Japan & GB & Frankreich & Niederlande & Belgien & Italien & Österreich \\
\hline & Importwert, in Mio. $€$ & 123,0 & 111,1 & 63,8 & 47,5 & 41,0 & 28,0 & 27,7 & 26,9 & 25,1 \\
\hline & Unit Value, in $€$ je kg & 3,71 & . & 5,81 & 4,90 & 3,72 & 2,96 & 3,74 & 3,54 & 3,74 \\
\hline \multirow[t]{3}{*}{ Holztüren u.dgl. } & Länder & USA & GB & Deutschland & Japan & Hongkong & Kanada & Dänemark & Norwegen & Irland \\
\hline & Importwert, in Mio. $€$ & 468,9 & 224,1 & 143,9 & 124,1 & 76,4 & 60,7 & 55,9 & 55,4 & 46,4 \\
\hline & Unit Value, in $€ \mathrm{je} \mathrm{kg}$ & & 1,94 & 2,49 & 4,76 & & . & 2,97 & 3,84 & 2,33 \\
\hline \multirow[t]{3}{*}{ Holzschindeln } & Länder & USA & Kanada & Frankreich & Deutschland & Italien & GB & Mexiko & Jamaika & Österreich \\
\hline & Importwert, in Mio. $€$ & 253,7 & 11,5 & 3,6 & 2,4 & 1,8 & 1,7 & 1,2 & 1,1 & 1,1 \\
\hline & Unit Value, in $€$ je $\mathrm{kg}$ & 1,12 & & 0,63 & 1,17 & 0,84 & 1,85 & 0,96 & 1,25 & 1,10 \\
\hline \multirow[t]{3}{*}{ And. Bautischlerarbeit } & Länder & USA & Deutschland & Japan & GB & Österreich & Italien & Frankreich & Schweiz & Niederlande \\
\hline & Importwert, in Mio. $€$ & 956,9 & 618,4 & 385,2 & 173,1 & 167,4 & 164,5 & 159,5 & 124,3 & 109,5 \\
\hline & Unit Value, in $€$ je kg & & 1,43 & 1,28 & 1,96 & 1,48 & 1,12 & 1,33 & 2,00 & \\
\hline
\end{tabular}

Quelle: UNO (WIFO-Datenbank) 
Tabelle 54: Die wichtigsten Importeure von vorgefertigten Häusern und Möbeln aus Holz [2000]

\begin{tabular}{|c|c|c|c|c|c|c|c|c|c|c|}
\hline \multirow[t]{3}{*}{ Vorgefertigte Häuser } & Länder & Deutschland & USA & Japan & Frankreich & Schweiz & Mexiko & Kanada & Belgien & Österreich \\
\hline & Importwert, in Mio. $€$ & 408,8 & 316,7 & 246,6 & 185,1 & 106,4 & 96,6 & 86,2 & 84,9 & 84,6 \\
\hline & Unit Value, in $€$ je kg & 1,10 & 2,44 & 1,44 & 1,43 & 2,12 & 2,45 & & & 1,28 \\
\hline \multirow[t]{3}{*}{ Sitze a.n.g. mit Holzgestell } & Länder & USA & Deutschland & Frankreich & GB & Japan & Belgien & Niederlande & Schweiz & Kanada \\
\hline & Importwert, in Mio. $€$ & $2.398,2$ & $1.009,0$ & 542,0 & 531,3 & 514,6 & 279,3 & 275,9 & 251,0 & 239,8 \\
\hline & Unit Value, in $€$ je kg & $\cdot$ & 3,06 & 6,61 & 5,19 & 4,62 & 5,63 & 4,63 & 7,93 & . \\
\hline \multirow[t]{3}{*}{ Büromöbel aus Holz } & Länder & USA & GB & Frankreich & Deutschland & Belgien & Schweiz & Norwegen & Kanada & Hongkong \\
\hline & Importwert, in Mio. $€$ & 774,8 & 169,6 & 141,9 & 119,5 & 92,5 & 86,9 & 81,4 & 66,0 & 54,7 \\
\hline & Unit Value, in $€$ je kg & & 3,79 & 3,03 & 1,79 & 3,86 & 3,16 & 5,26 & . & \\
\hline \multirow[t]{3}{*}{ Küchenmöbel aus Holz } & Länder & USA & Niederlande & Frankreich & Schweiz & Deutschland & GB & Belgien & Norwegen & Österreich \\
\hline & Importwert, in Mio. $€$ & 721,8 & 161,6 & 159,2 & 139,2 & 97,2 & 87,6 & 82,5 & 70,4 & 69,2 \\
\hline & Unit Value, in $€$ je kg & . & 2,73 & 2,35 & 5,54 & 2,18 & 3,34 & 2,93 & 3,54 & 2,72 \\
\hline \multirow[t]{3}{*}{ Schlafzimmermöbel aus Holz } & Länder & USA & Deutschland & Frankreich & GB & Schweiz & Niederlande & Belgien & Österreich & Hongkong \\
\hline & Importwert, in Mio. $€$ & $1.827,0$ & 441,6 & 270,1 & 240,9 & 156,3 & 139,3 & 118,3 & 113,1 & 110,8 \\
\hline & Unit Value, in $€$ je kg & . & 1,97 & 2,00 & 2,68 & 2,75 & 2,13 & 1,94 & 2,16 & \\
\hline \multirow[t]{3}{*}{ Andere Möbel aus Holz } & Länder & USA & Deutschland & Japan & Frankreich & GB & Hongkong & Niederlande & Belgien & Schweiz \\
\hline & Importwert, in Mio. $€$ & $3.955,1$ & $1.260,5$ & 928,6 & 839,7 & 825,5 & 485,7 & 412,2 & 353,1 & 326,2 \\
\hline & Unit Value, in $€$ je kg & & 2,47 & 2,84 & 2,45 & 2,88 & & 2,38 & 2,71 & 3,15 \\
\hline
\end{tabular}

Quelle: UNO (WIFO-Datenbank) 
Abbildung 6: Holzwaren, roh und einfach behandelt, Index 1998=100 [1990 - 2001]

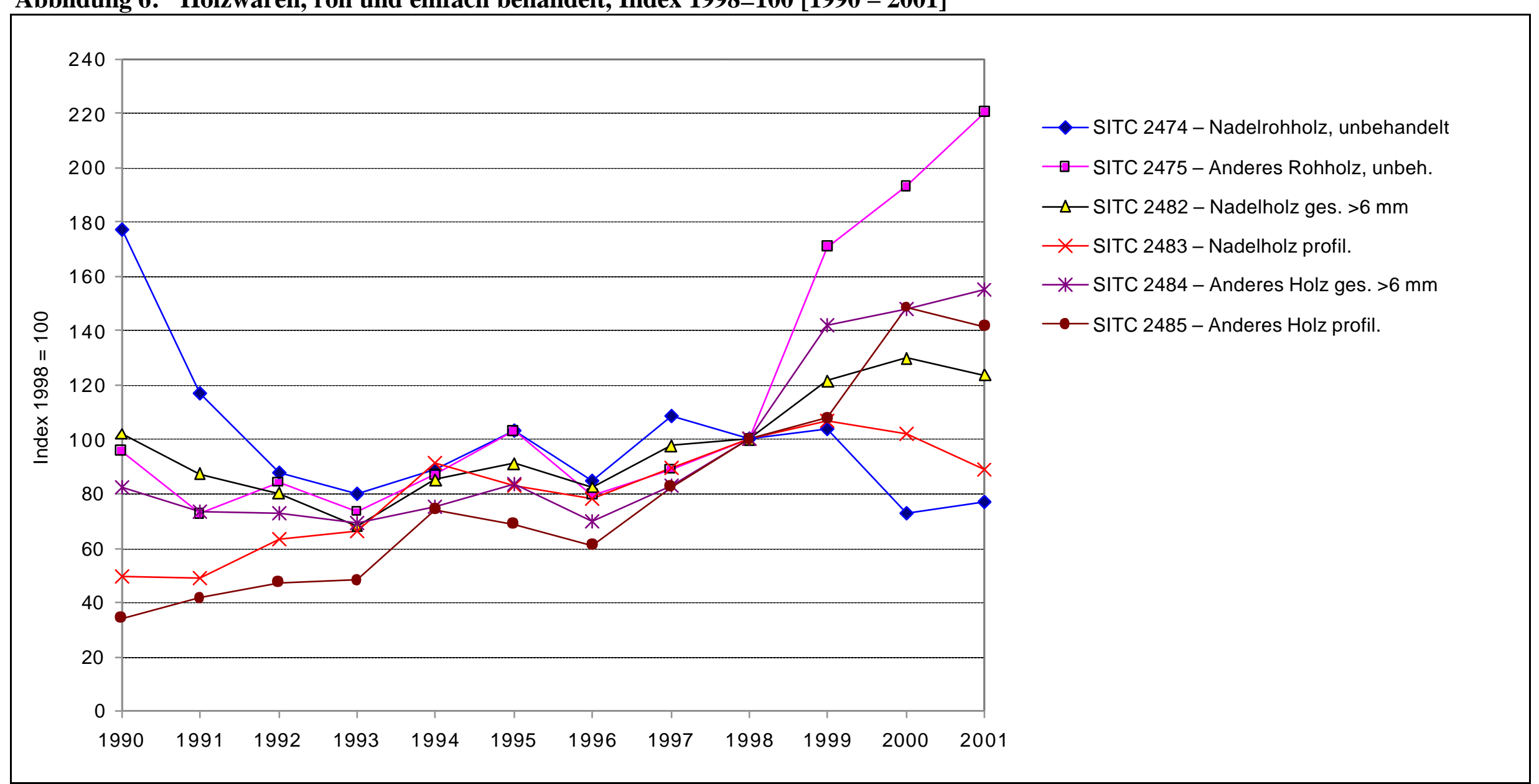

Quelle: Statistik Austria (WIFO-Datenbank) 


\section{Abbildung 7: Export von Holzwaren, roh und einfach behandelt, Unit Values [1990 - 2001]}

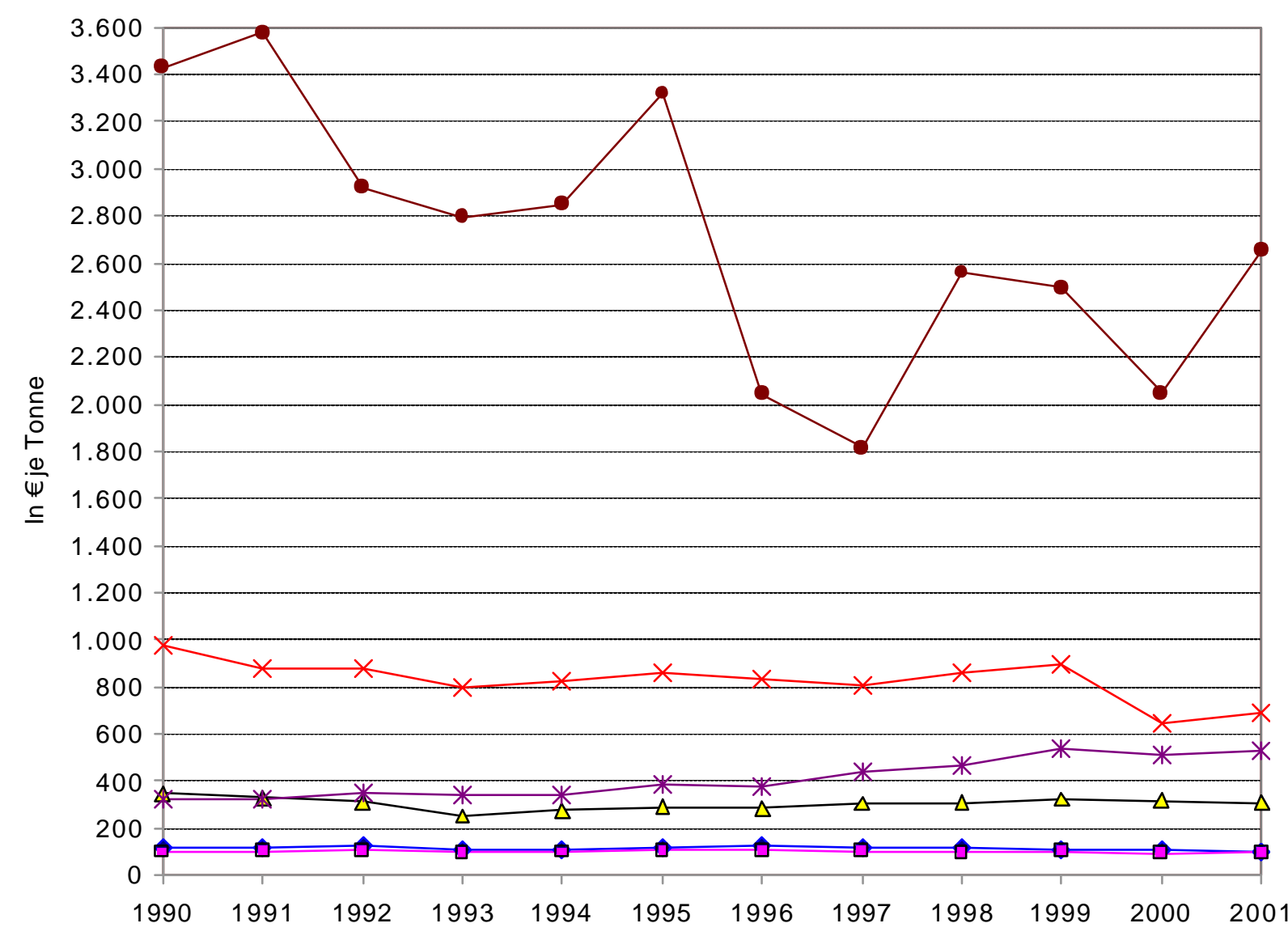

$\multimap$ SITC 2474 - Nadelrohholz, unbehandelt

- - SITC 2475 - Anderes Rohholz, unbehandelt

$\triangle$ SITC 2482 - Nadelholz ges. $>6$ mm

$x$ SITC 2483 - Nadelholz profil.

* SITC 2484 - Anderes Holz ges. >6 mm

$\longrightarrow$ SITC 2485 - Anderes Holz profil.

Quelle: Statistik Austria (WIFO-Datenbank) 
Abbildung 8: Holzwaren, roh und einfach behandelt, RCA-Werte [1990 - 2001]

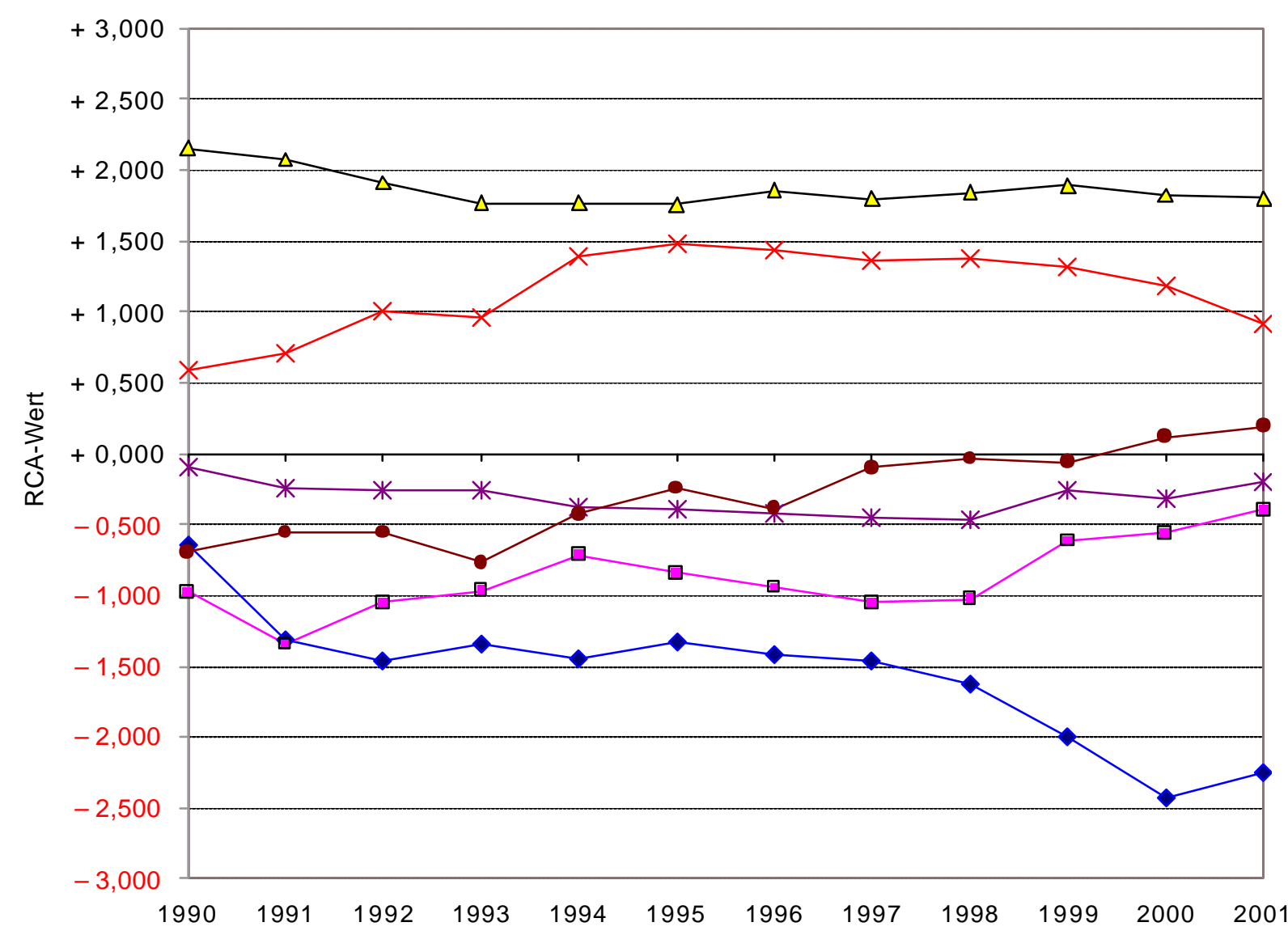

$\multimap$ SITC 2474 - Nadelrohholz, unbehandelt

$\square$ SITC 2475 - Anderes Rohholz,

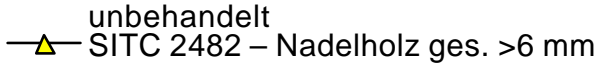

$\leftarrow$ SITC 2483 - Nadelholz profil.

* SITC 2484 - Anderes Holz ges. $>6$ mm

- SITC 2485 - Anderes Holz profil.

Quelle: Statistik Austria (WIFO-Datenbank) 
Abbildung 9: Exportwert von Furnieren und Platten aus Holz, Index $1998=100$ [1990 - 2001]

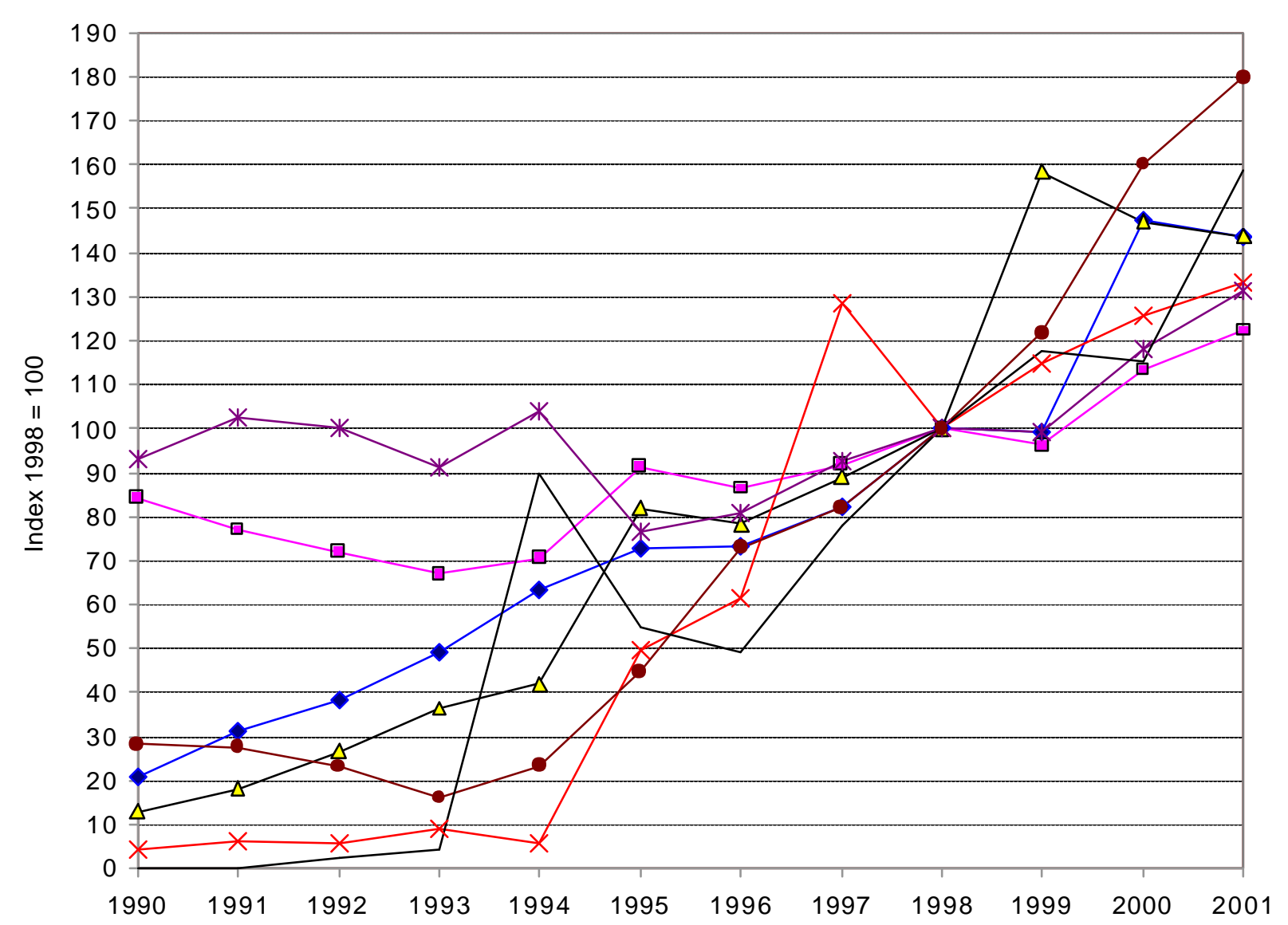

$\multimap$ SITC 63412 - Furniere aus and. Holz

—-SITC 63422 - Span- u.ä. Platten aus $\mathrm{Holz}$

$\checkmark$ SITC 63431 - Sperrholz mit Tropenholz

$\leftarrow$ SITC 63441 - And. Sperrholz a.

* SITC 63449 - Anderes Sperrholz u.ä.

$\longrightarrow$ a.n.g. 63451 - Faserplatten $>0,8 \mathrm{~g} / \mathrm{cm} 3$ ......... -

Quelle: Statistik Austria (WIFO-Datenbank) 
Abbildung 10: $\quad$ Export von Furnieren und Platten aus Holz, Unit Values [1990 - 2001]

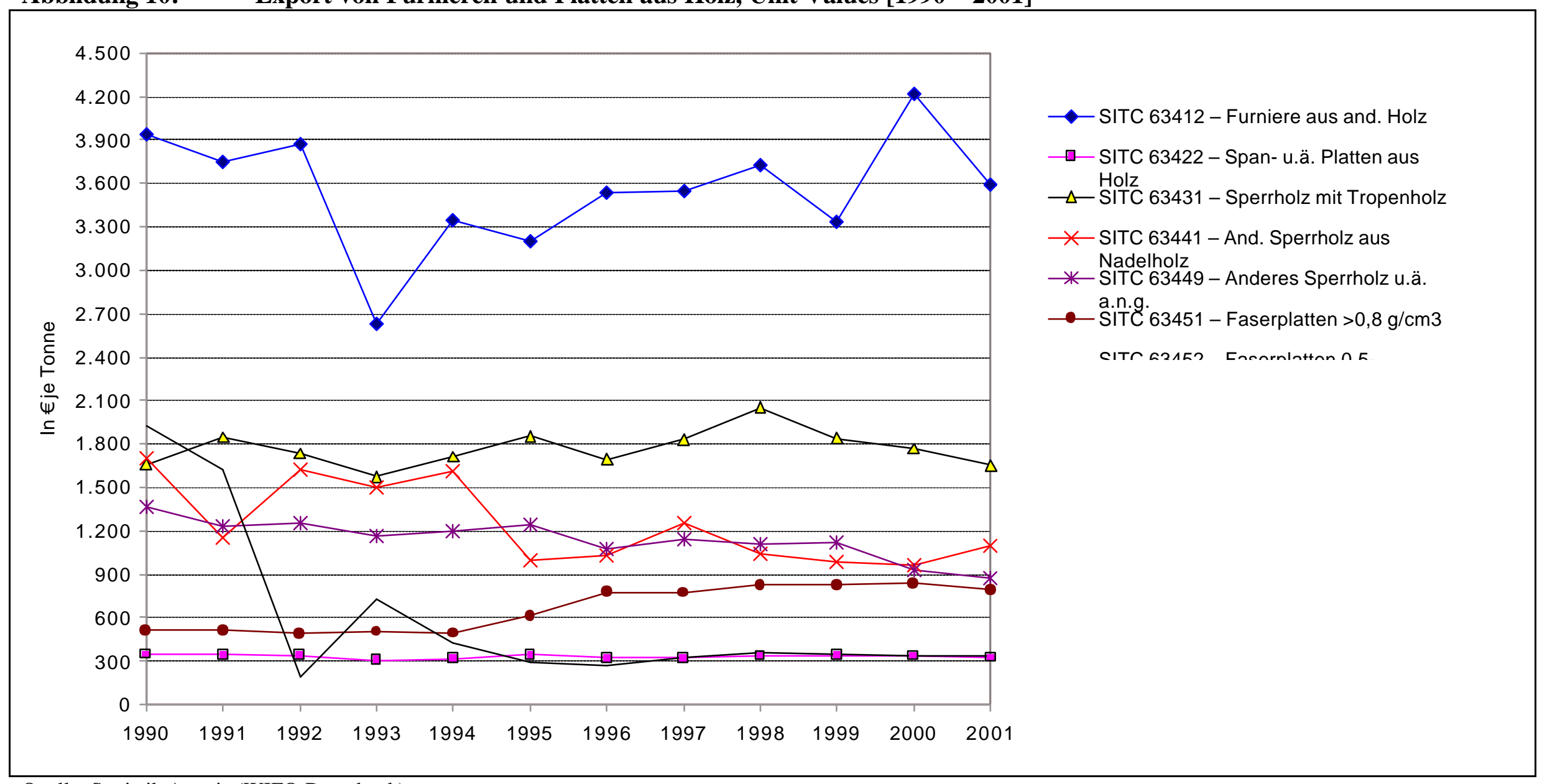

Quelle: Statistik Austria (WIFO-Datenbank) 
Abbildung 11: Furniere und Platten aus Holz, RCA-Werte [1990 - 2001]

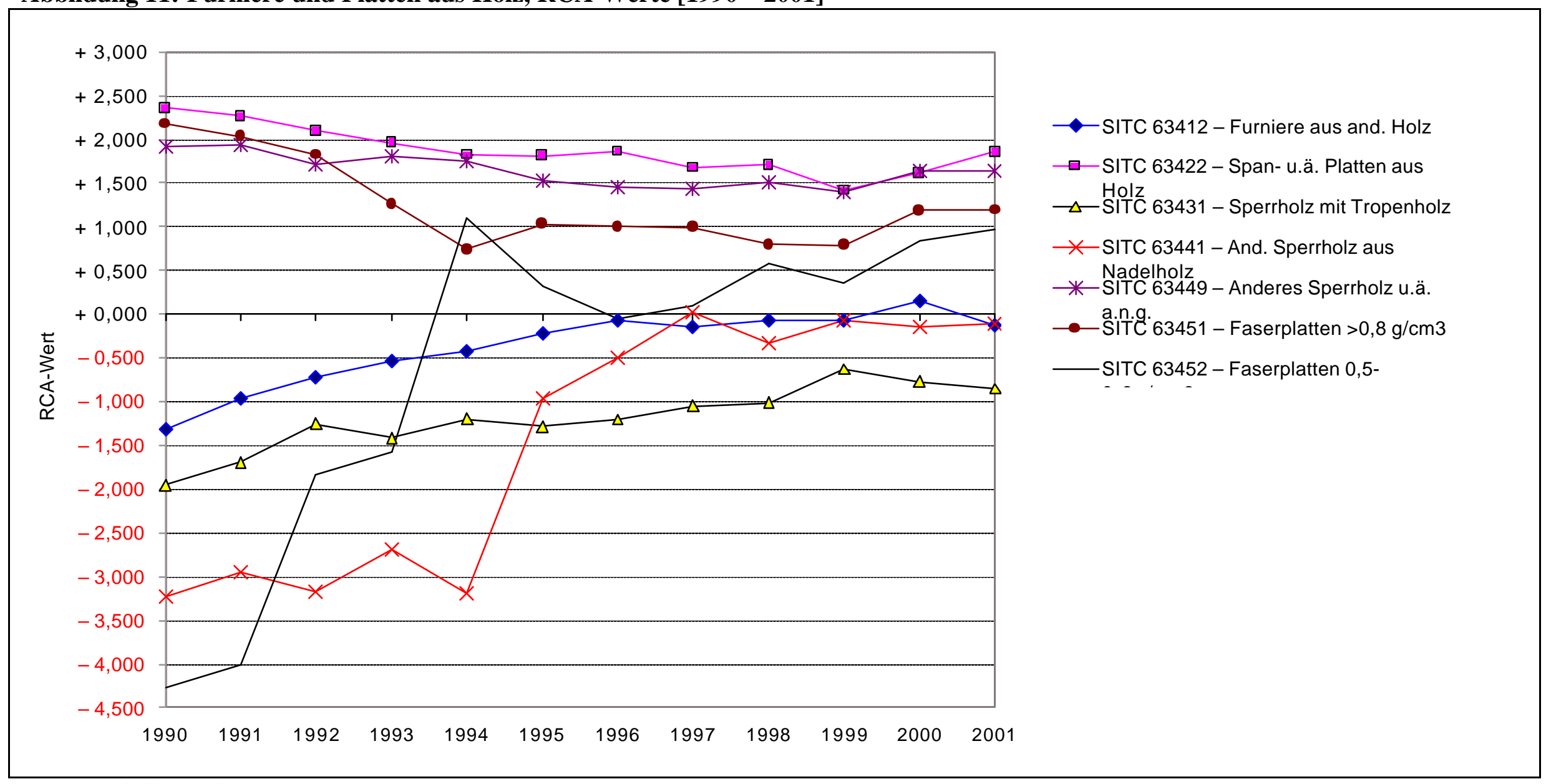

Quelle: Statistik Austria (WIFO-Datenbank) 
Abbildung 12: Exportwert von Holzwaren, Index 1998 = 100 [1990 - 2001]

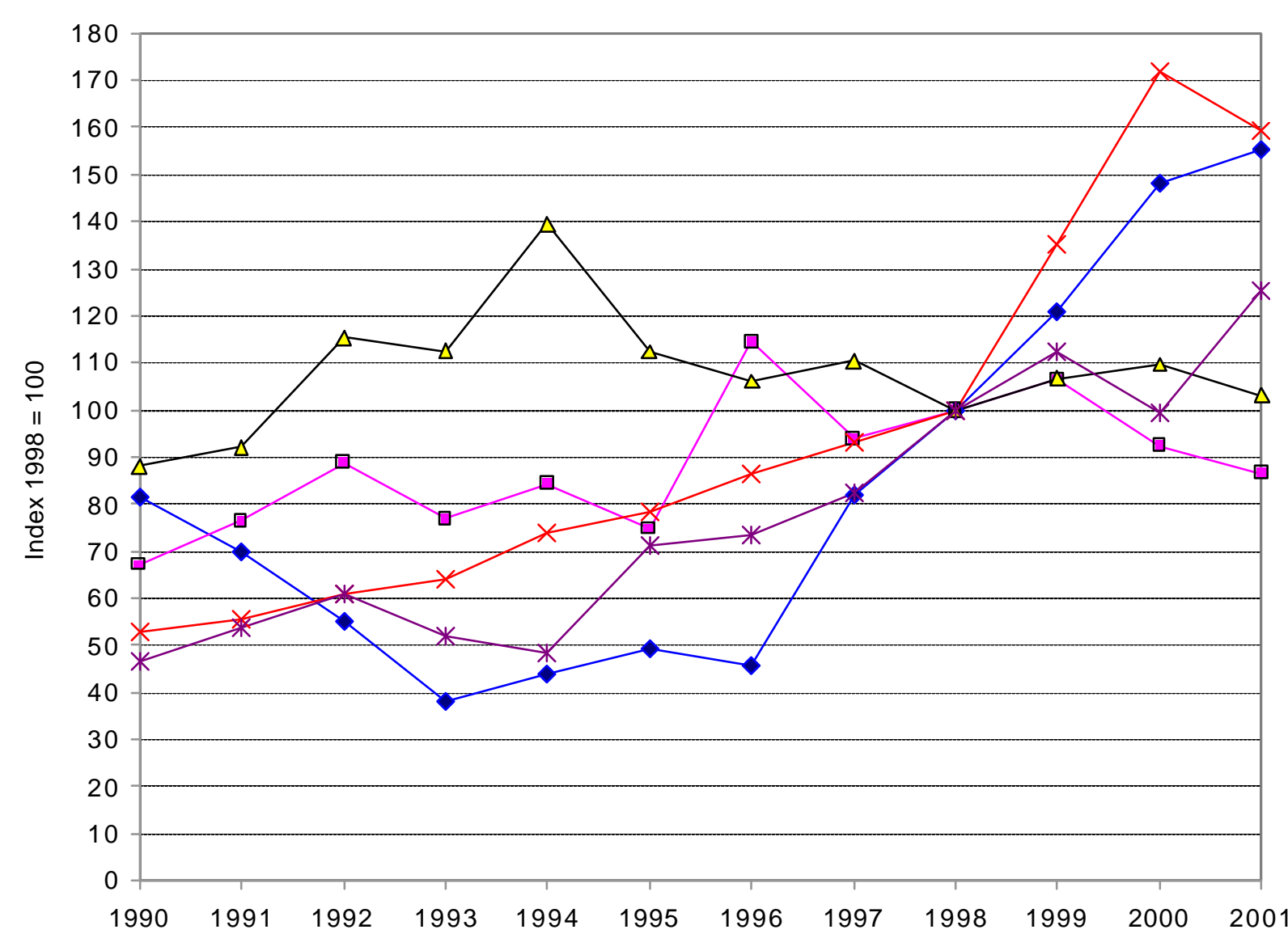

$\bullet$ SITC 63512 - Flachpaletten u.dgl.

—SITC 63531 - Holzfenster u.dgl. $\triangle \_$SITC 63532 - Holztüren u.dgl.

$\leftarrow$ SITC $63539-$ A. Bautischlerarbeit

* SITC 811 - Vorgefertigte Häuser

Quelle: Statistik Austria (WIFO-Datenbank) 
Abbildung 13: Export von Holzwaren, Unit Values [1990 - 2001]

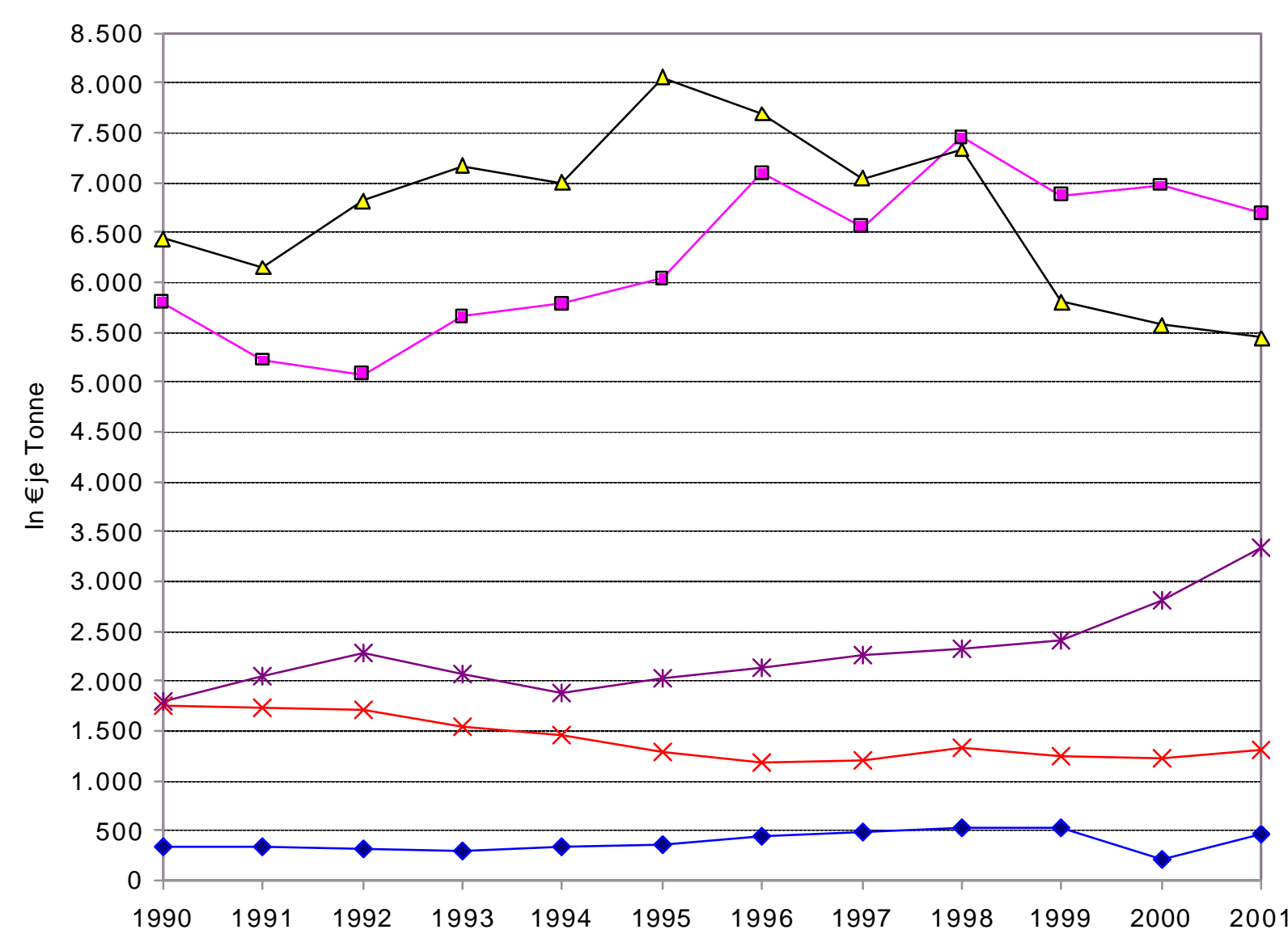

—SITC 63512 - Flachpaletten u.dgl.

घ-SITC 63531 - Holzfenster u.dgl.

$\triangle$-SITC 63532 - Holztüren u.dgl.

$\leftarrow$ SITC $63539-$ A. Bautischlerarbeit

* SITC 811 - Vorgefertigte Häuser

Quelle: Statistik Austria (WIFO-Datenbank) 
Abbildung 14: Holzwaren, RCA-Werte [1990 - 2001]

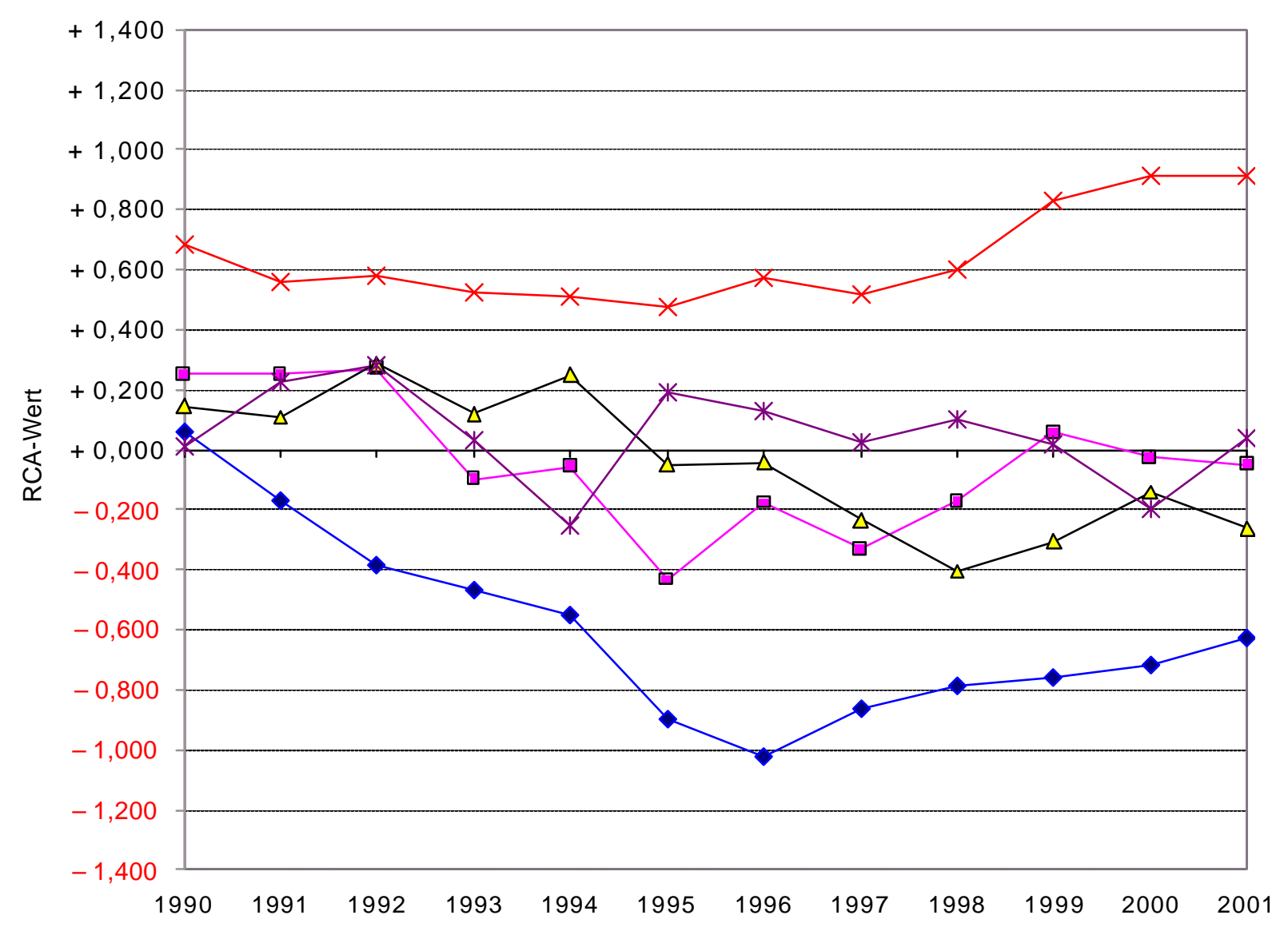

—SITC 63512 - Flachpaletten u.dgl.

$\square$ SITC 63531 - Holzfenster u.dgl.

$\triangle$ SITC 63532 - Holztüren u.dgl.

$\leftarrow$ SITC $63539-$ A. Bautischlerarbeit

* SITC 811 - Vorgefertigte Häuser

Quelle: Statistik Austria (WIFO-Datenbank) 
Abbildung 15: Exportwert von Möbeln aus Holz, Index 1998 = 100 [1990 - 2001]

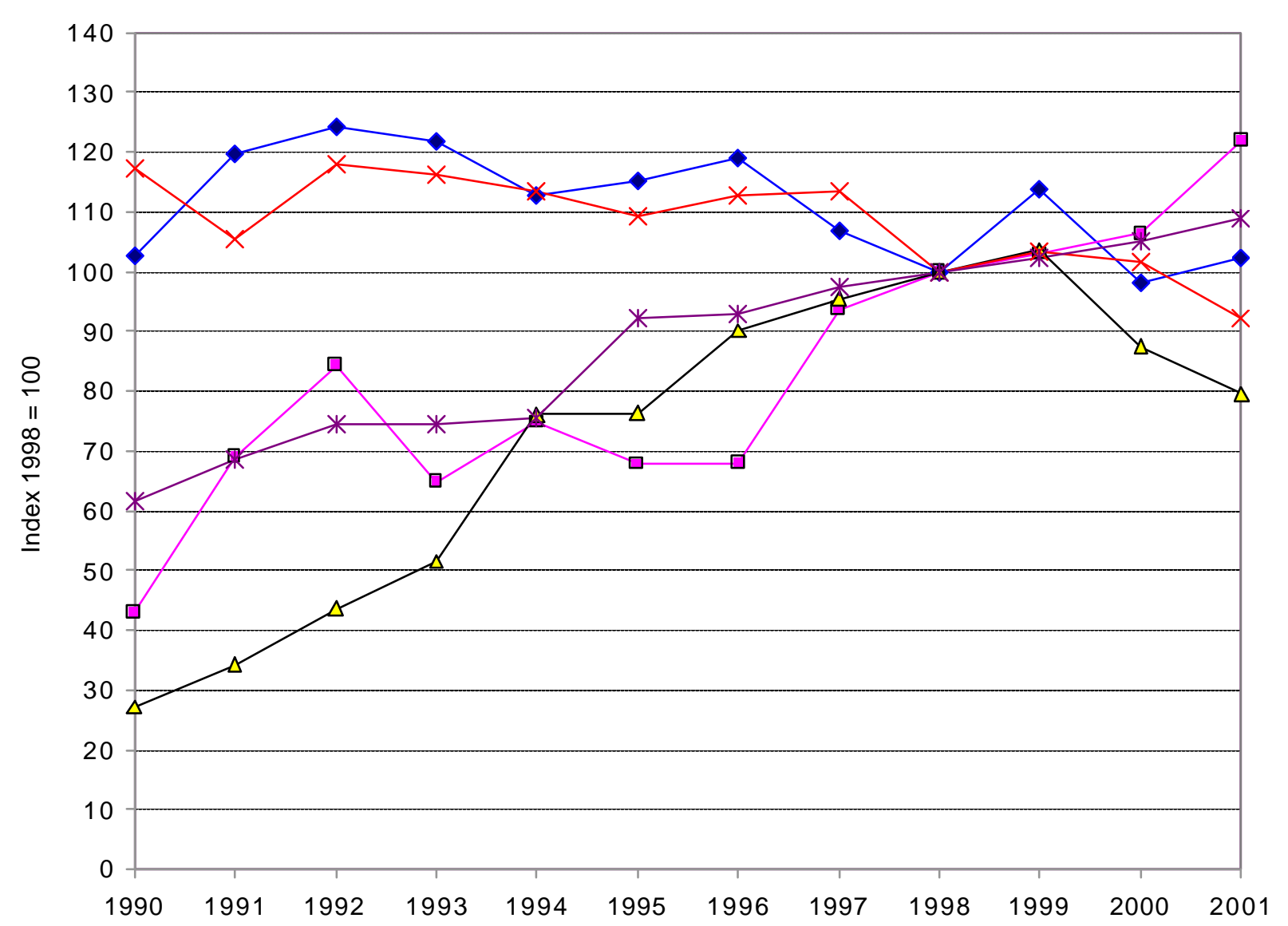

—SITC 82116 - Sitze a.n.g. m. Holzgestell —-SITC 82151 - Büromöbel aus Holz $\triangle$ SITC 82153 - Küchenmöbel aus Holz × SITC 82155 - Schlafzimmer aus Holz * SITC 82159 - Andere Möbel aus Holz

Quelle: Statistik Austria (WIFO-Datenbank) 
Abbildung 16:

Export von Möbeln aus Holz, Unit Values [1990 - 2001]

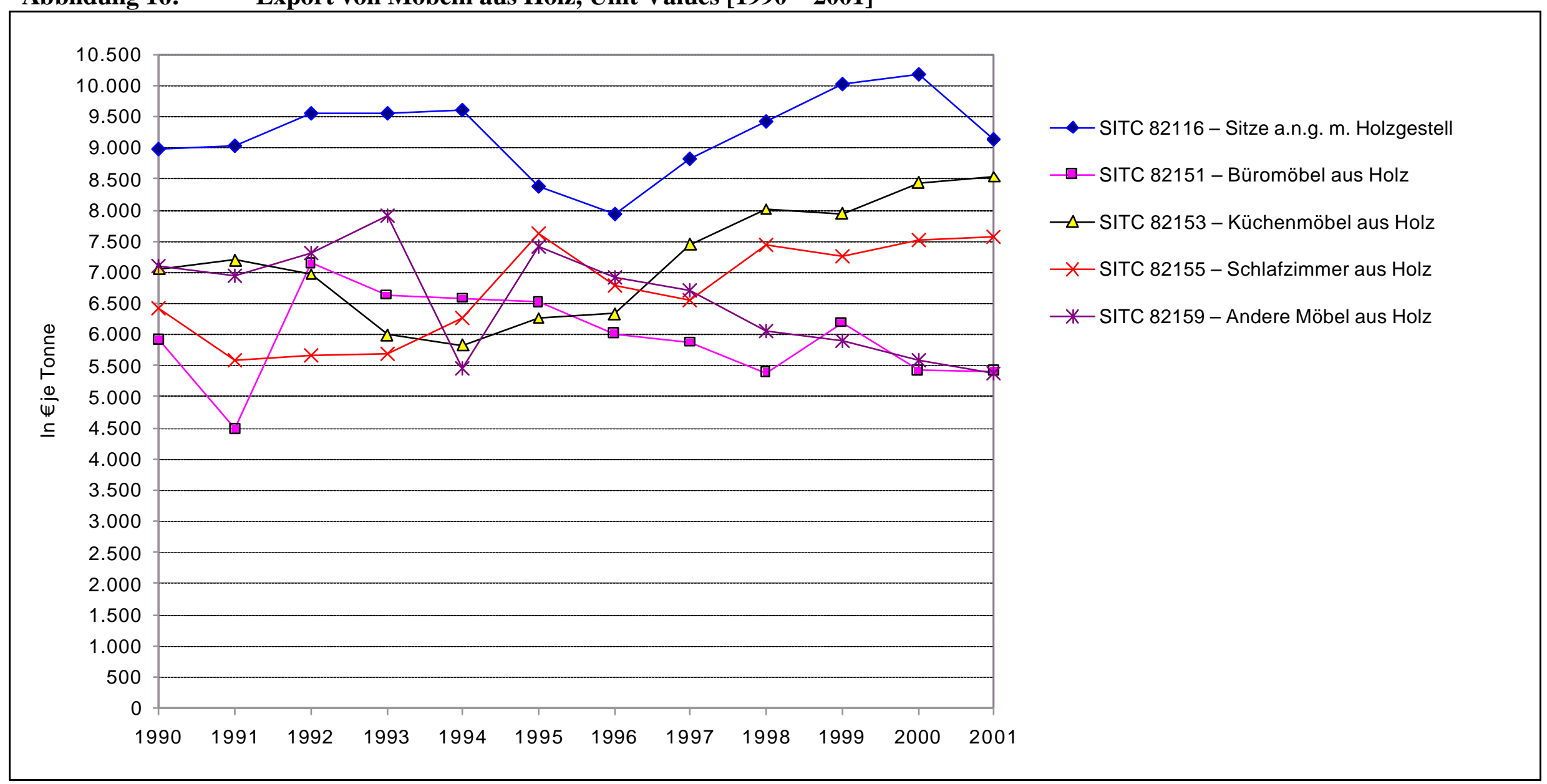

Quelle: Statistik Austria (WIFO-Datenbank) 
Möbel aus Holz, RCA-Werte [1990 - 2001]

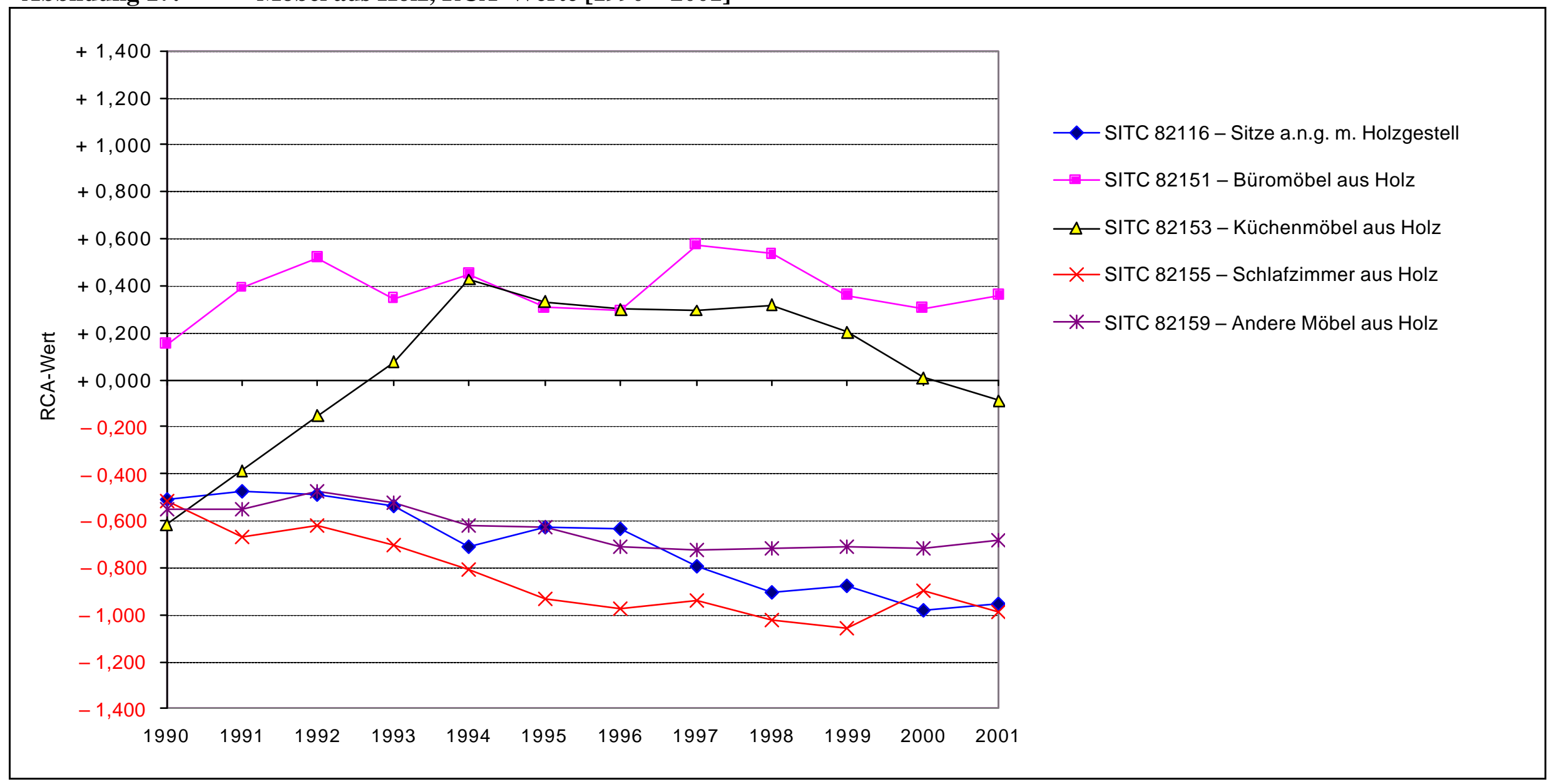

Quelle: Statistik Austria (WIFO-Datenbank) 
Abbildung 18: Dynamik der Exporte von Nadelrohholz, unbehandelt

\begin{tabular}{|c|c|c|c|}
\hline SITC 2474 & Exportwert & & RCA-Wert \\
\hline & \multicolumn{2}{|c|}{ Durchschnittliches jährl. Wachstum in \% } & \\
\hline & $1990 / 1997$ & $1997 / 2000$ & 2000 \\
\hline Russland & . & $+20,8$ & $+4,450$ \\
\hline USA & $-4,0$ & $-5,4$ & $+2,261$ \\
\hline Neuseeland & $+26,2$ & $-1,9$ & $+8,793$ \\
\hline Kanada & $-6,4$ & $+80,0$ & $+0,096$ \\
\hline Deutschland & $-4,8$ & $+2,7$ & $+0,027$ \\
\hline Schweiz & $+0,6$ & $+36,2$ & $+3,575$ \\
\hline Estland & . & $+24,6$ & $+2,335$ \\
\hline Frankreich & $-12,0$ & $+60,4$ & $+1,067$ \\
\hline Tschechien & . & $-7,2$ & $+0,941$ \\
\hline Lettland & . & $+30,9$ & $+3,641$ \\
\hline Österreich & $-6,1$ & $-12,4$ & $-2,428$ \\
\hline
\end{tabular}

Quelle: UNO (WIFO-Datenbank)
Exportwert Nadelrohholz, unbehandelt In Mio. € 2000

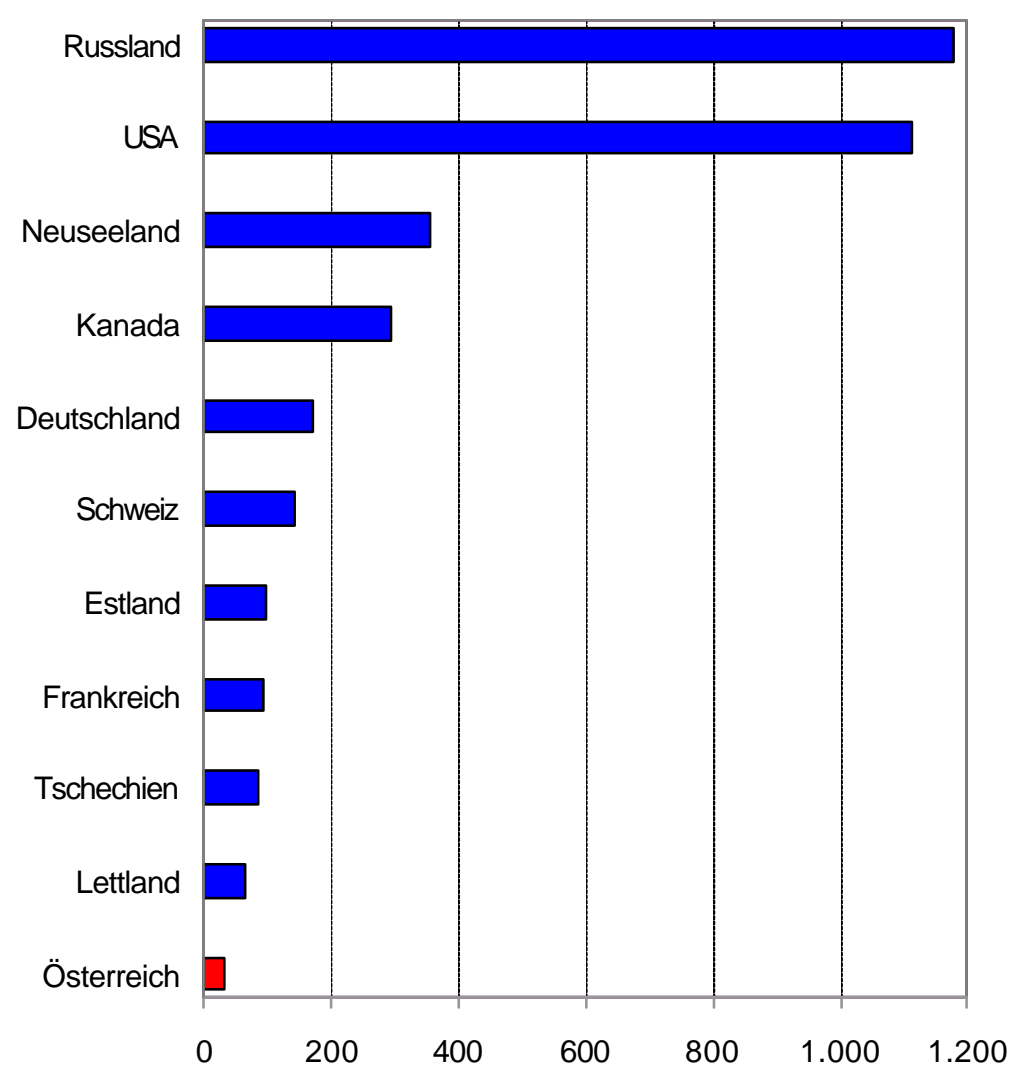


Abbildung 19: Dynamik der Exporte von anderem Rohholz, unbehandelt

\begin{tabular}{|c|c|c|c|}
\hline SITC 2475 & Exportwert & & RCA-Wert \\
\hline & \multicolumn{2}{|c|}{ Durchschnittliches jährl. Wachstum in \% } & \\
\hline & $1990 / 1997$ & $1997 / 2000$ & 2000 \\
\hline Malaysia & $-6,4$ & $-1,4$ & $+2,163$ \\
\hline USA & $+4,5$ & $+17,1$ & $+2,731$ \\
\hline Frankreich & $-4,7$ & $+16,5$ & $+0,211$ \\
\hline Russland & . & $+4,7$ & $+6,840$ \\
\hline Deutschland & $+9,8$ & $+44,0$ & $+0,164$ \\
\hline Lettland & . & $+31,0$ & $+3,154$ \\
\hline Kanada & $+20,6$ & $+14,3$ & $-1,274$ \\
\hline Belgien & $+4,3$ & $+3,2$ & $-0,629$ \\
\hline Dänemark & $+19,0$ & $+33,9$ & $+0,543$ \\
\hline Estland & . & $+11,8$ & $+2,857$ \\
\hline Österreich & $-0,4$ & $+29,7$ & $-0,561$ \\
\hline
\end{tabular}

Quelle: UNO (WIFO-Datenbank)
Exportwert anderes Rohholz, unbehandelt In Mio. €

2000

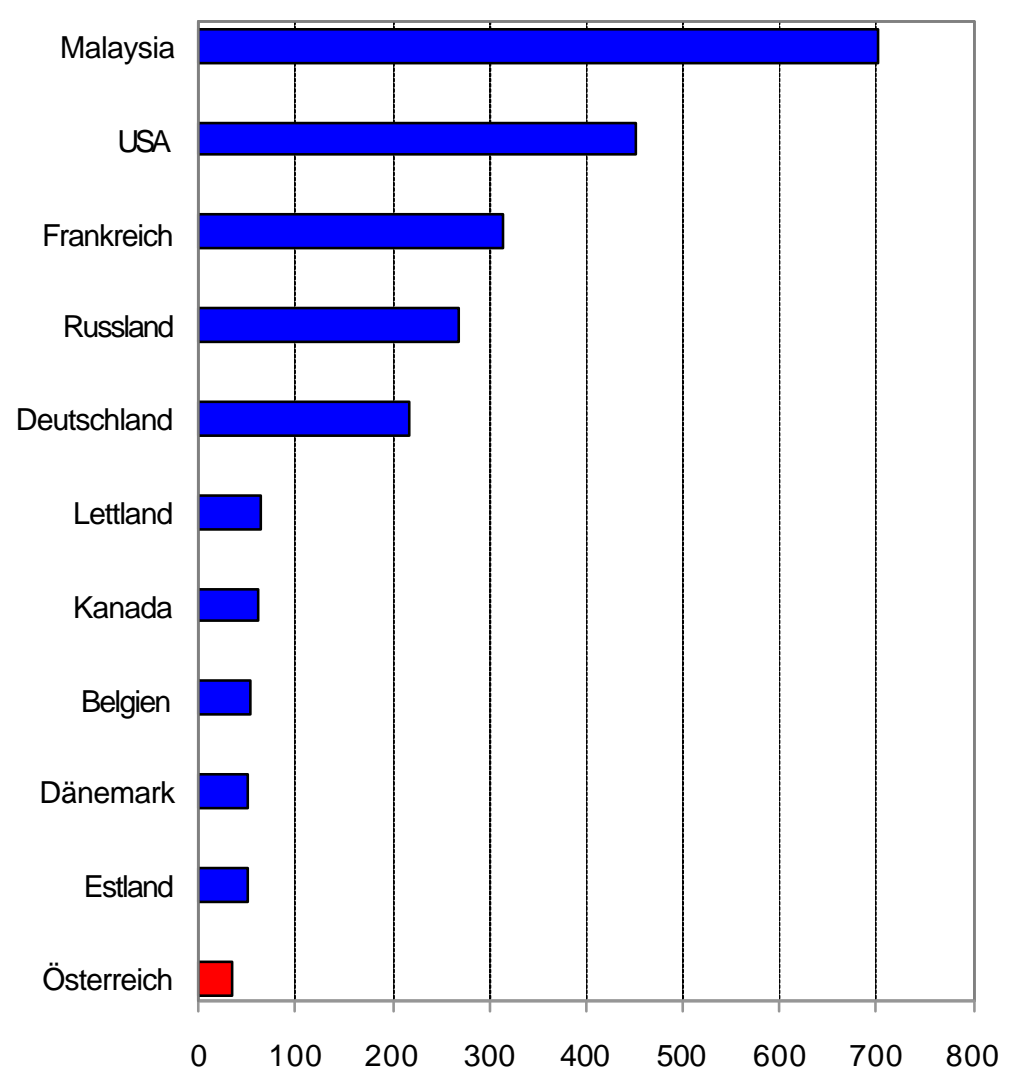


Abbildung 20: Dynamik der Exporte von Nadelholz ges.

\section{$>6 \mathrm{~mm}$}

\begin{tabular}{|c|c|c|c|}
\hline SITC 2482 & Exportwert & & RCA-Wert \\
\hline & \multicolumn{2}{|c|}{ Durchschnittliches jährl. Wachstum in \% } & \\
\hline & $1990 / 1997$ & $1997 / 2000$ & 2000 \\
\hline Kanada & $+12,3$ & $+1,9$ & $+3,741$ \\
\hline Schweden & $+6,6$ & $-1,0$ & $+4,095$ \\
\hline Finnland & $+6,8$ & $+2,5$ & $+3,635$ \\
\hline Österreich & $+0,0$ & $+10,1$ & $+1,824$ \\
\hline USA & $-1,1$ & $-5,7$ & $-1,649$ \\
\hline Russland & . & $+10,9$ & $+6,138$ \\
\hline Deutschland & $+8,4$ & $+14,1$ & $-0,694$ \\
\hline Lettland & . & $+16,9$ & $+3,949$ \\
\hline Neuseeland & $+16,4$ & $+14,3$ & $+3,200$ \\
\hline Chile & $+12,0$ & $+10,2$ & $+4,937$ \\
\hline
\end{tabular}

Quelle: UNO (WIFO-Datenbank)

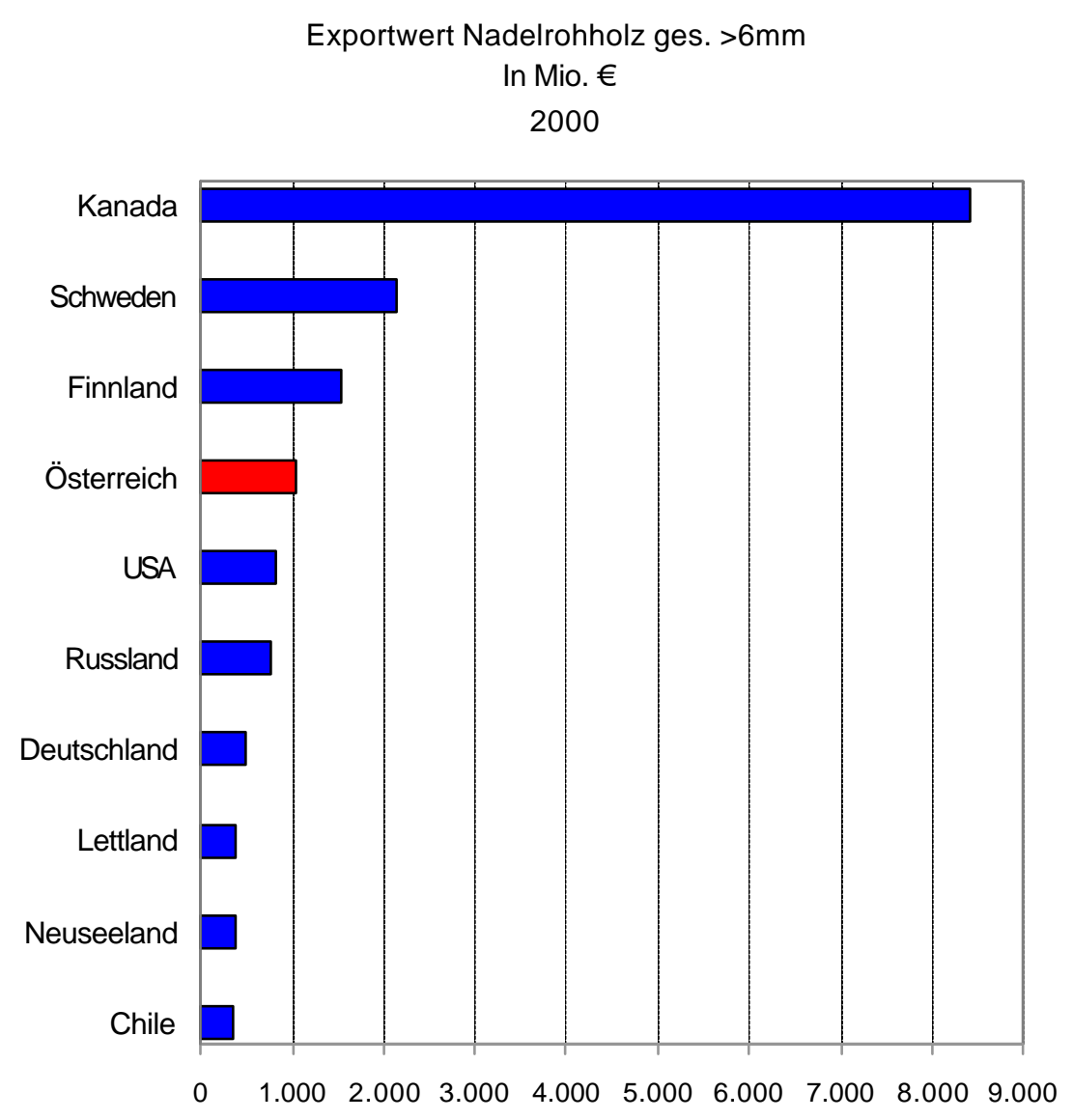


Abbildung 21: Dynamik der Exporte von Nadelholz profiliert

\begin{tabular}{|c|c|c|c|}
\hline SITC 2483 & Exportwert & & RCA-Wert \\
\hline & \multicolumn{2}{|c|}{ Durchschnittliches jährl. Wachstum in \% } & \\
\hline & 1990/1997 & $1997 / 2000$ & 2000 \\
\hline Kanada & $+5,1$ & $+11,6$ & $+0,779$ \\
\hline Chile & $+36,7$ & $+5,5$ & $+4,546$ \\
\hline USA & $+10,2$ & $-3,0$ & $-1,452$ \\
\hline Mexiko & $+6,3$ & $-8,8$ & $+1,608$ \\
\hline Österreich & $+9,5$ & $+4,5$ & $+1,186$ \\
\hline Italien & $+17,3$ & $+5,3$ & $+0,370$ \\
\hline Neuseeland & $+31,4$ & $+12,9$ & $+4,849$ \\
\hline Deutschland & $-2,0$ & $+2,0$ & $-0,495$ \\
\hline Finnland & $+9,8$ & $+6,8$ & $+3,209$ \\
\hline Schweden & $+0,4$ & $-0,4$ & $+1,925$ \\
\hline
\end{tabular}

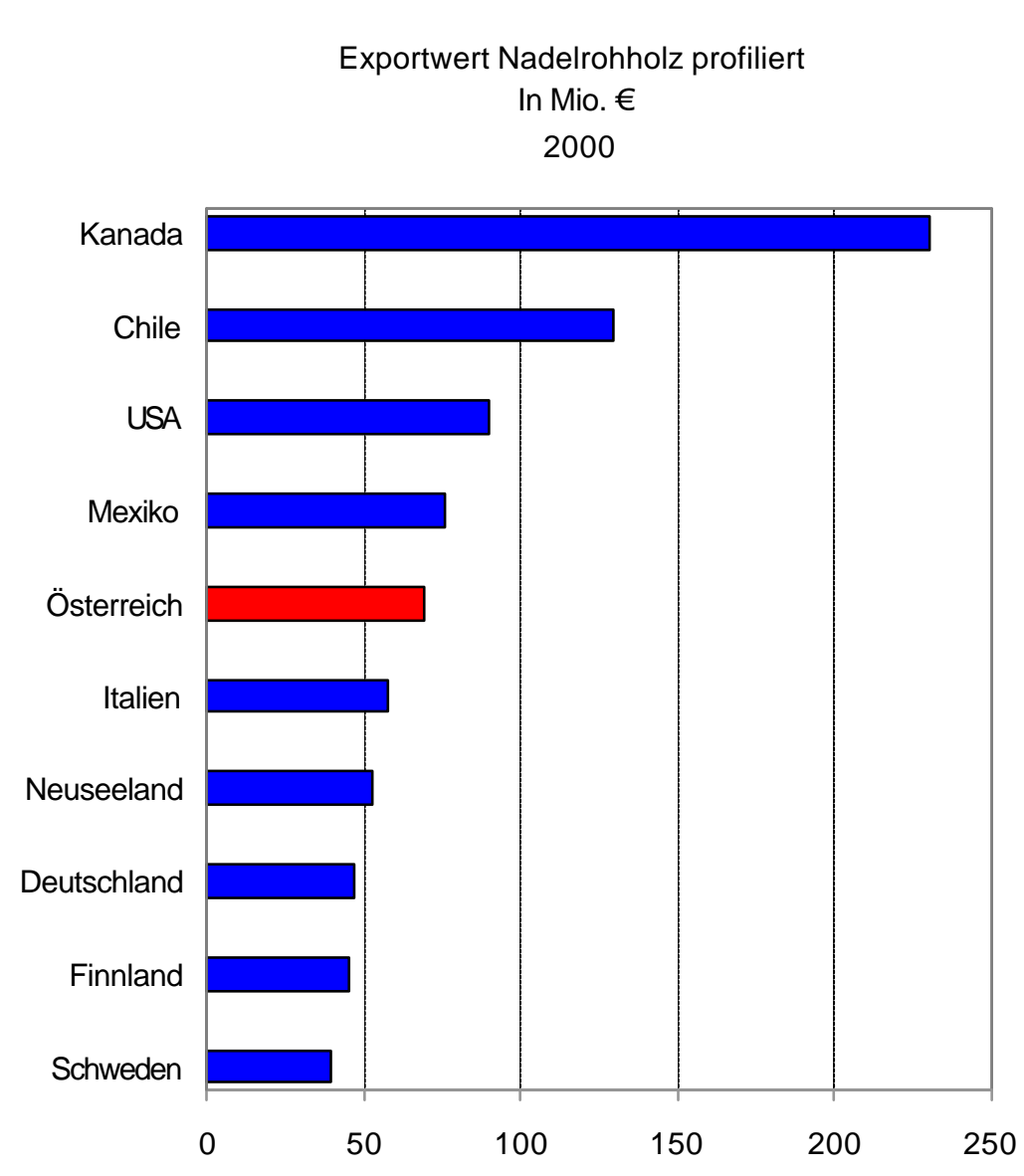


Abbildung 22: Dynamik der Exporte von anderem Holz ges. $>6 \mathrm{~mm}$

\begin{tabular}{|c|c|c|c|}
\hline SITC 2484 & Exportwert & & RCA-Wert \\
\hline & \multicolumn{2}{|c|}{ Durchschnittliches jährl. Wachstum in \% } & \\
\hline & 1990/1997 & $1997 / 2000$ & 2000 \\
\hline USA & $+10,4$ & $+7,0$ & $+1,563$ \\
\hline Malaysia & $+0,5$ & $+0,4$ & $+2,236$ \\
\hline Kanada & $+15,3$ & $+10,5$ & $+0,008$ \\
\hline Brasilien & $+13,5$ & $+12,4$ & $+4,068$ \\
\hline Deutschland & $+3,1$ & $+23,9$ & $-0,223$ \\
\hline Elfenbeinküste & . & $+16,4$ & $+7,112$ \\
\hline Frankreich & $-1,8$ & $+7,3$ & $-0,371$ \\
\hline Belgien & $+11,4$ & $+15,4$ & $-0,549$ \\
\hline Rumänien & $+16,3$ & $+30,1$ & $+4,456$ \\
\hline China & . & $+3,4$ & $-1,962$ \\
\hline Österreich & $+0,7$ & $+21,4$ & $-0,311$ \\
\hline
\end{tabular}

Quelle: UNO (WIFO-Datenbank)

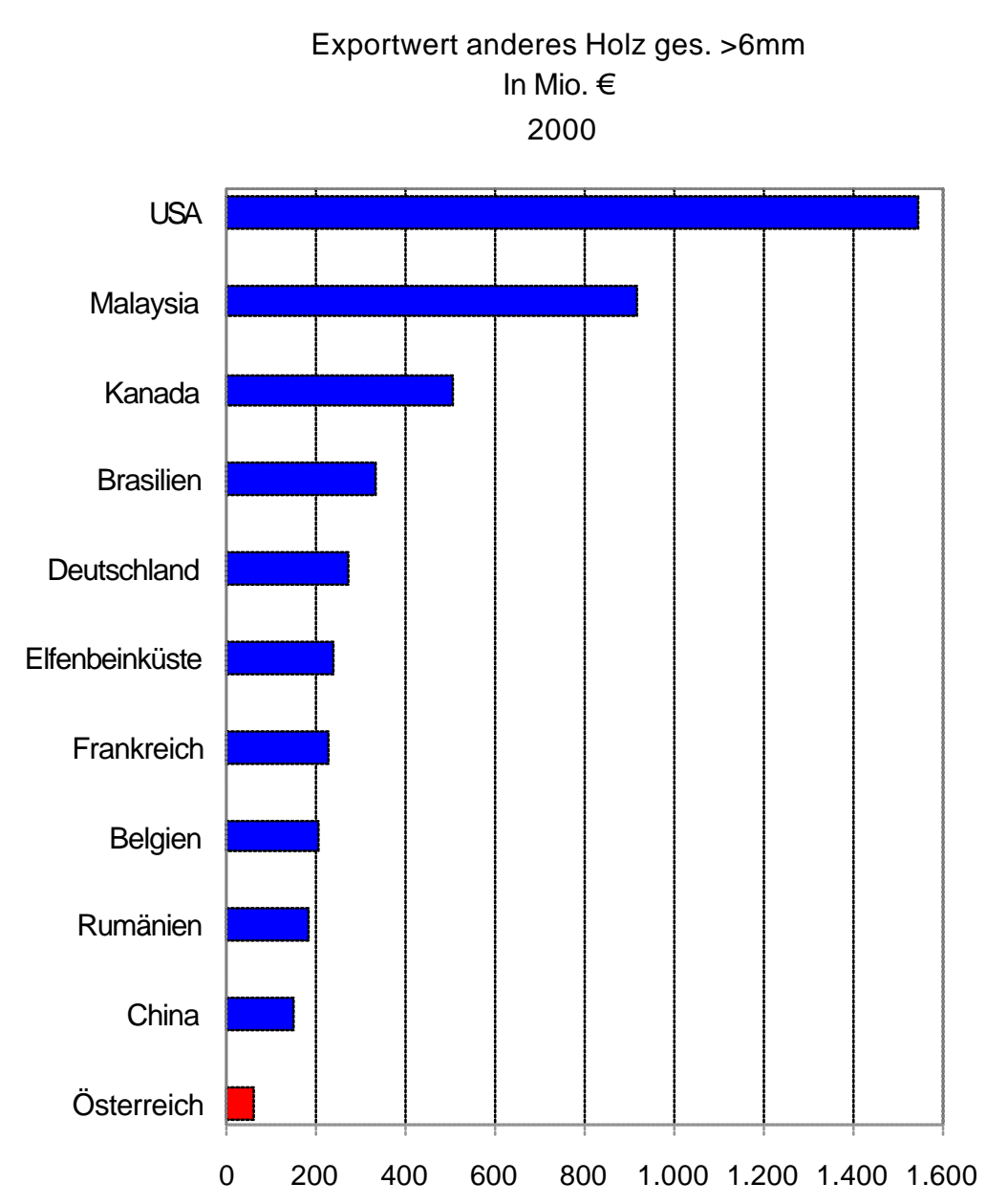


Abbildung 23: Dynamik der Exporte von anderem Holz profiliert

\begin{tabular}{|c|c|c|c|}
\hline SITC 2485 & Exportwert & & RCA-Wert \\
\hline & \multicolumn{2}{|c|}{ Durchschnittliches jährl. Wachstum in \% } & \\
\hline & $1990 / 1997$ & $1997 / 2000$ & 2000 \\
\hline Indonesien & $+1,6$ & $+22,6$ & $+4,764$ \\
\hline Malaysia & $+5,6$ & $+0,3$ & $+2,486$ \\
\hline Italien & $+18,2$ & $+11,7$ & $+0,294$ \\
\hline USA & $+8,9$ & $+10,6$ & $-0,274$ \\
\hline Brasilien & $+38,9$ & $+37,5$ & $+4,582$ \\
\hline China & 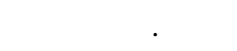 & $+28,2$ & $+2,374$ \\
\hline Belgien & $+18,7$ & $+37,7$ & $-0,383$ \\
\hline Kanada & $+10,1$ & $+22,0$ & $-0,534$ \\
\hline Thailand & $+13,3$ & $+18,2$ & $+3,878$ \\
\hline Frankreich & $+7,3$ & $+8,6$ & $+0,362$ \\
\hline Österreich & $+14,1$ & $+21,8$ & $+0,119$ \\
\hline
\end{tabular}

Quelle: UNO (WIFO-Datenbank)
Exportwert anderes Holz profiliert

In Mio. $€$

2000

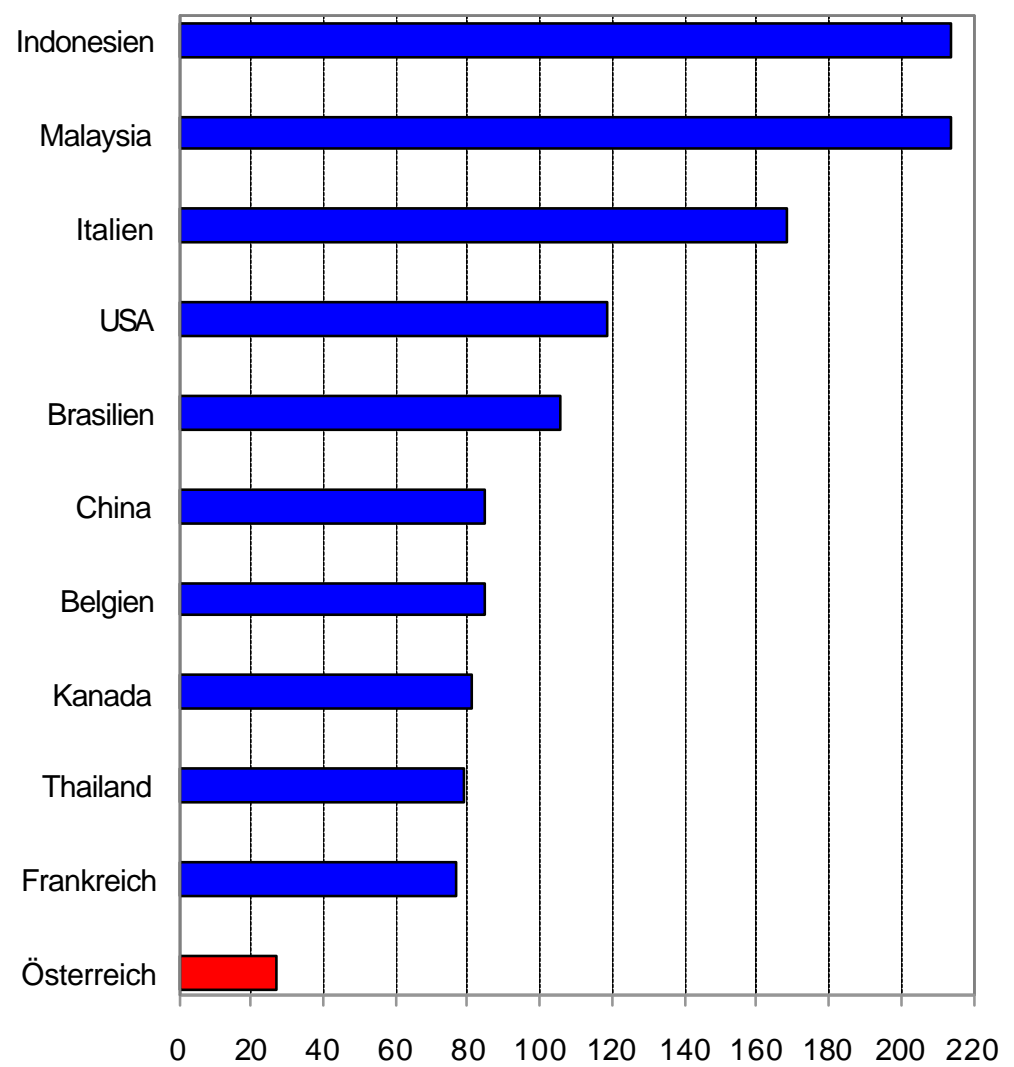


Abbildung 24: Dynamik der Exporte von Furnieren aus Nadelholz

\begin{tabular}{|c|c|c|c|}
\hline SITC 63411 & Exportwert & & RCA-Wert \\
\hline & \multicolumn{2}{|c|}{ Durchschnittliches jährl. Wachstum in \% } & \\
\hline & $1990 / 1997$ & $1997 / 2000$ & 2000 \\
\hline Kanada & $+19,8$ & $+21,0$ & $+1,908$ \\
\hline USA & $+7,6$ & $+28,4$ & $-0,483$ \\
\hline Finnland & $+12,0$ & $+7,6$ & $+3,666$ \\
\hline Chile & $+61,5$ & $-4,5$ & $+0,000$ \\
\hline Portugal & $-8,0$ & $-2,7$ & $+1,146$ \\
\hline Spanien & $+1,7$ & $+14,9$ & $-0,547$ \\
\hline Italien & $-0,6$ & $+7,9$ & $-0,817$ \\
\hline Brasilien & $+86,4$ & $-22,3$ & $+1,771$ \\
\hline Schweden & $+3,2$ & $+1,1$ & $-0,563$ \\
\hline Großbritannien & $-8,3$ & $+32,8$ & $-0,655$ \\
\hline Österreich & $-6,4$ & $-6,5$ & $-0,001$ \\
\hline
\end{tabular}

Quelle: UNO (WIFO-Datenbank)

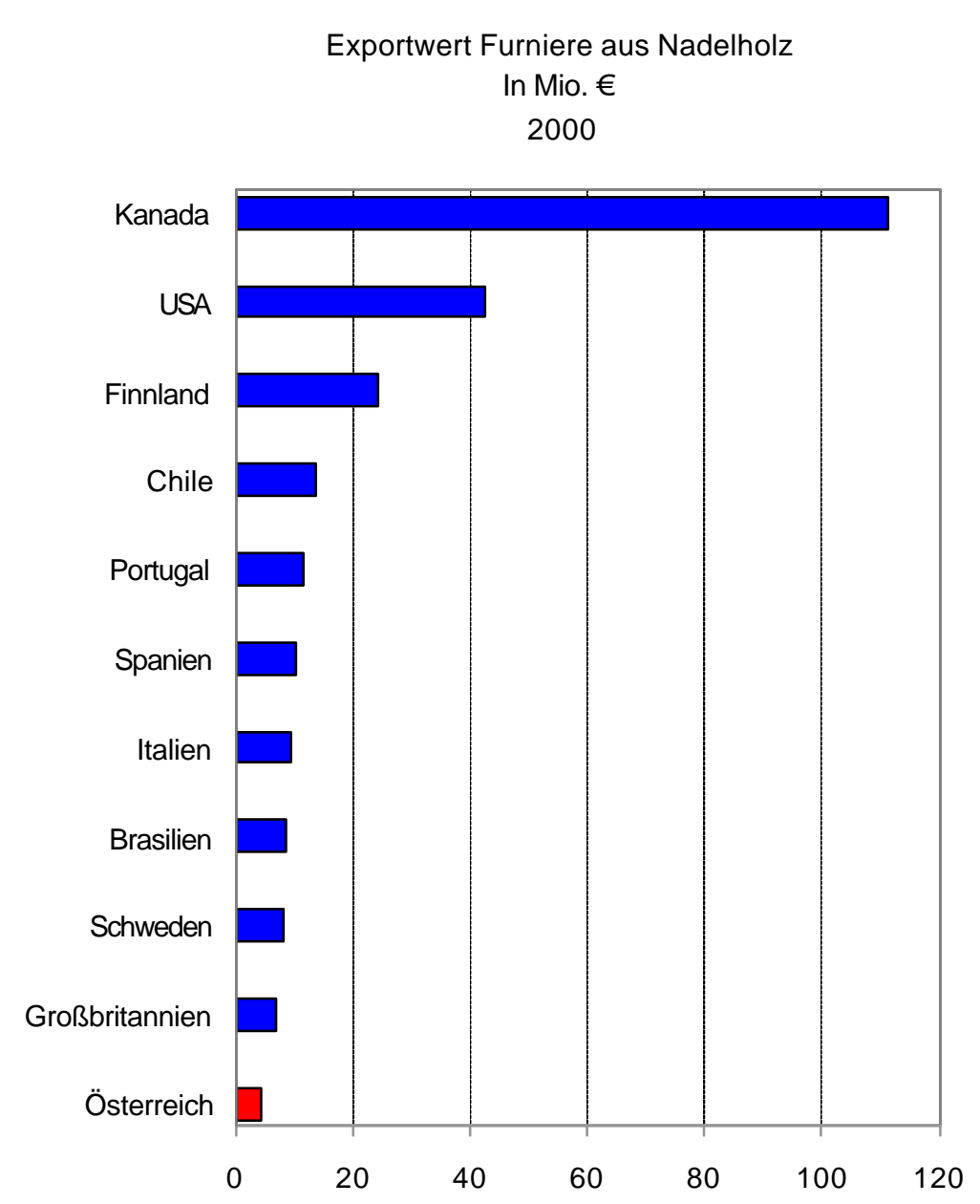


Abbildung 25: Dynamik der Exporte von Furnieren aus anderem Holz

\begin{tabular}{|c|c|c|c|}
\hline SITC 63412 & Exportwert & & RCA-Wert \\
\hline & \multicolumn{2}{|c|}{ Durchschnittliches jährl. Wachstum in \% } & \\
\hline & $1990 / 1997$ & $1997 / 2000$ & 2000 \\
\hline USA & $+11,0$ & $+11,6$ & $+0,707$ \\
\hline Deutschland & $+6,5$ & $+7,5$ & $-0,005$ \\
\hline Kanada & $+9,8$ & $+13,8$ & $+0,362$ \\
\hline Malaysia & $+22,1$ & $+0,3$ & $+1,749$ \\
\hline Frankreich & $+6,1$ & $+5,9$ & $+0,471$ \\
\hline Italien & $+14,8$ & $+4,3$ & $-0,989$ \\
\hline China & . & $+16,1$ & $-1,379$ \\
\hline Spanien & $+10,8$ & $+13,6$ & $-0,665$ \\
\hline Elfenbeinküste & & $+6,5$ & $+9,341$ \\
\hline Belgien & $+1,7$ & $+0,2$ & $-0,105$ \\
\hline Österreich & $+22,7$ & $+21,5$ & $+0,149$ \\
\hline
\end{tabular}

Quelle: UNO (WIFO-Datenbank)
Exportwert Furniere aus anderem Holz

In Mio. $€$

2000

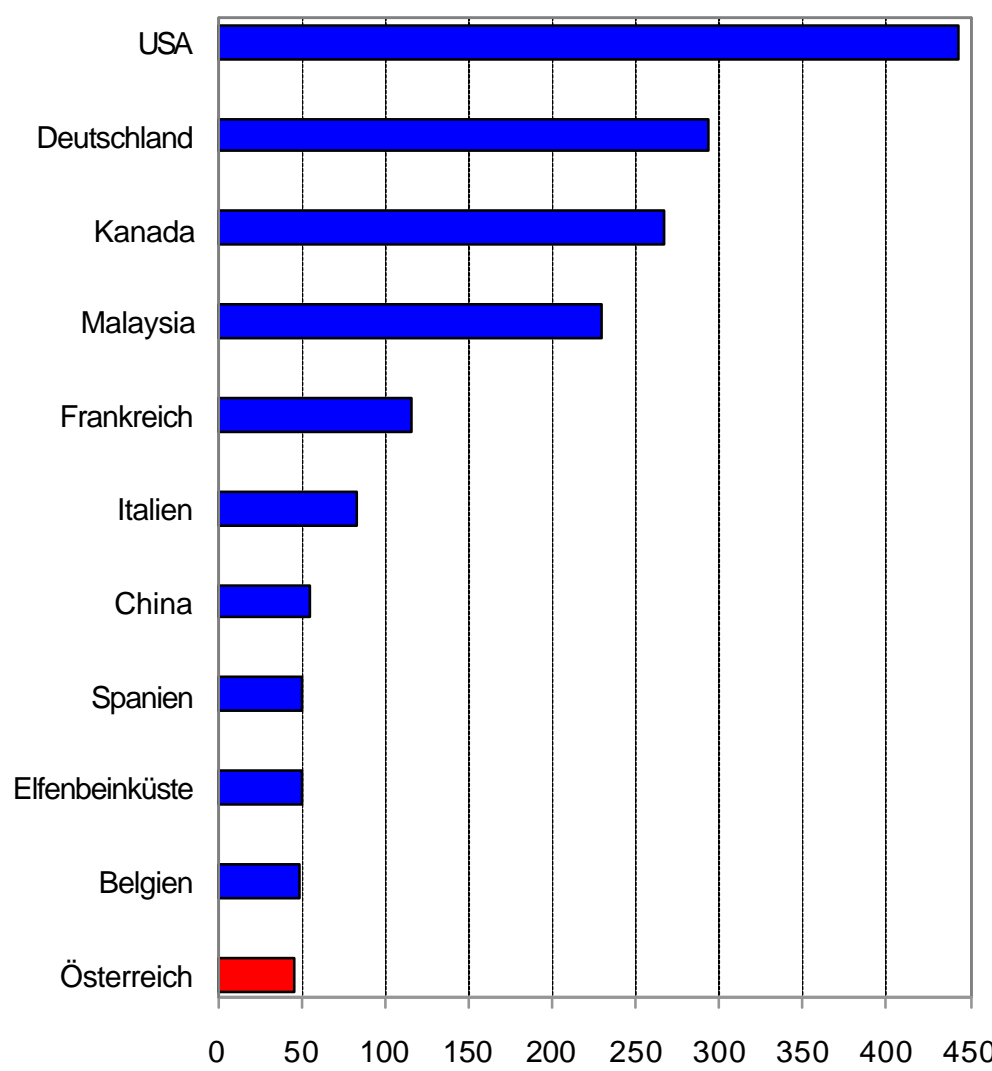


Abbildung 26: Dynamik der Exporte von Span- u.a. Platten aus Holz

\begin{tabular}{|c|c|c|c|}
\hline SITC 63422 & Exportwert & & RCA-Wert \\
\hline & \multicolumn{2}{|c|}{ Durchschnittliches jährl. Wachstum in \% } & \\
\hline & 1990/1997 & $1997 / 2000$ & 2000 \\
\hline Kanada & $+25,2$ & $+29,5$ & $+2,544$ \\
\hline Deutschland & $+4,8$ & $+4,9$ & $+0,348$ \\
\hline Belgien & $+2,7$ & $+1,6$ & $+1,538$ \\
\hline Österreich & $+1,9$ & $+7,4$ & $+1,615$ \\
\hline Frankreich & $+12,0$ & $+4,5$ & $+0,327$ \\
\hline USA & $+7,8$ & $+17,1$ & $-1,715$ \\
\hline Schweden & $+6,5$ & $+31,2$ & $+0,525$ \\
\hline Polen & . & $+53,7$ & $+1,430$ \\
\hline Italien & $+9,6$ & $+25,9$ & $-0,128$ \\
\hline Schweiz & $+4,3$ & $-5,7$ & $+0,171$ \\
\hline Kanada & $+25,2$ & $+29,5$ & $+2,544$ \\
\hline
\end{tabular}

Quelle: UNO (WIFO-Datenbank)
Exportwert Span- u.ä. Platten aus Holz In Mio. € 2000

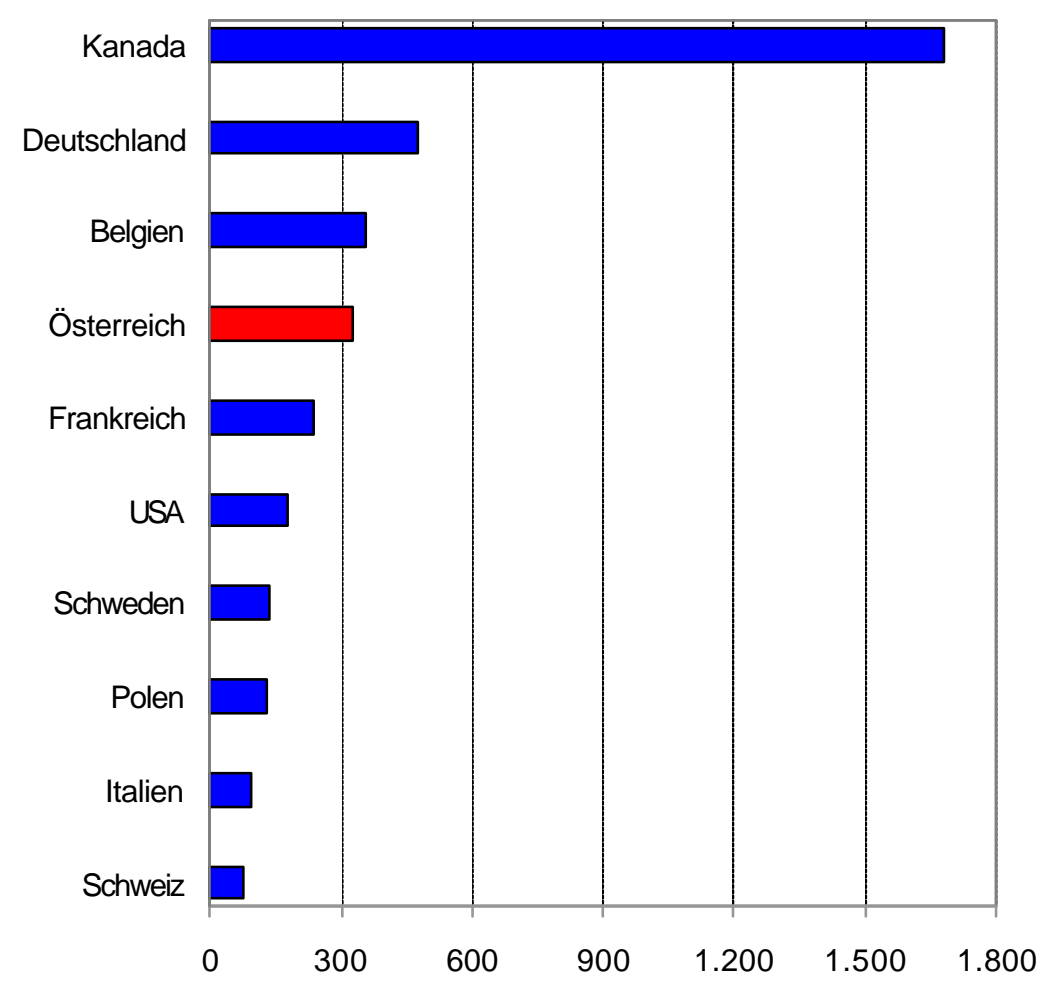


Abbildung 27: Dynamik der Exporte von Sperrholz mit Tropenholz

\begin{tabular}{|c|c|c|c|}
\hline SITC 63431 & Exportwert & & RCA-Wert \\
\hline & \multicolumn{2}{|c|}{ Durchschnittliches jährl. Wachstum in \% } & \\
\hline & $1990 / 1997$ & $1997 / 2000$ & 2000 \\
\hline Indonesien & $+2,8$ & $-11,5$ & $+6,813$ \\
\hline Malaysia & $+27,1$ & $-7,0$ & $+5,964$ \\
\hline Finnland & $-1,6$ & $+2,8$ & $+3,261$ \\
\hline Russland & . & $+8,1$ & $+4,669$ \\
\hline Kanada & $+18,8$ & $+22,1$ & $+0,881$ \\
\hline Brasilien & $+18,4$ & $+6,1$ & $+6,232$ \\
\hline Frankreich & $+5,6$ & $+7,4$ & $+0,221$ \\
\hline Belgien & $+26,8$ & $-2,7$ & $-0,382$ \\
\hline Lettland & . & . & $+5,204$ \\
\hline Italien & $+18,3$ & $+5,3$ & $-0,669$ \\
\hline
\end{tabular}

Quelle: UNO (WIFO-Datenbank)
Exportwert Sperrholz mit Tropenholz
In Mio. €
2000

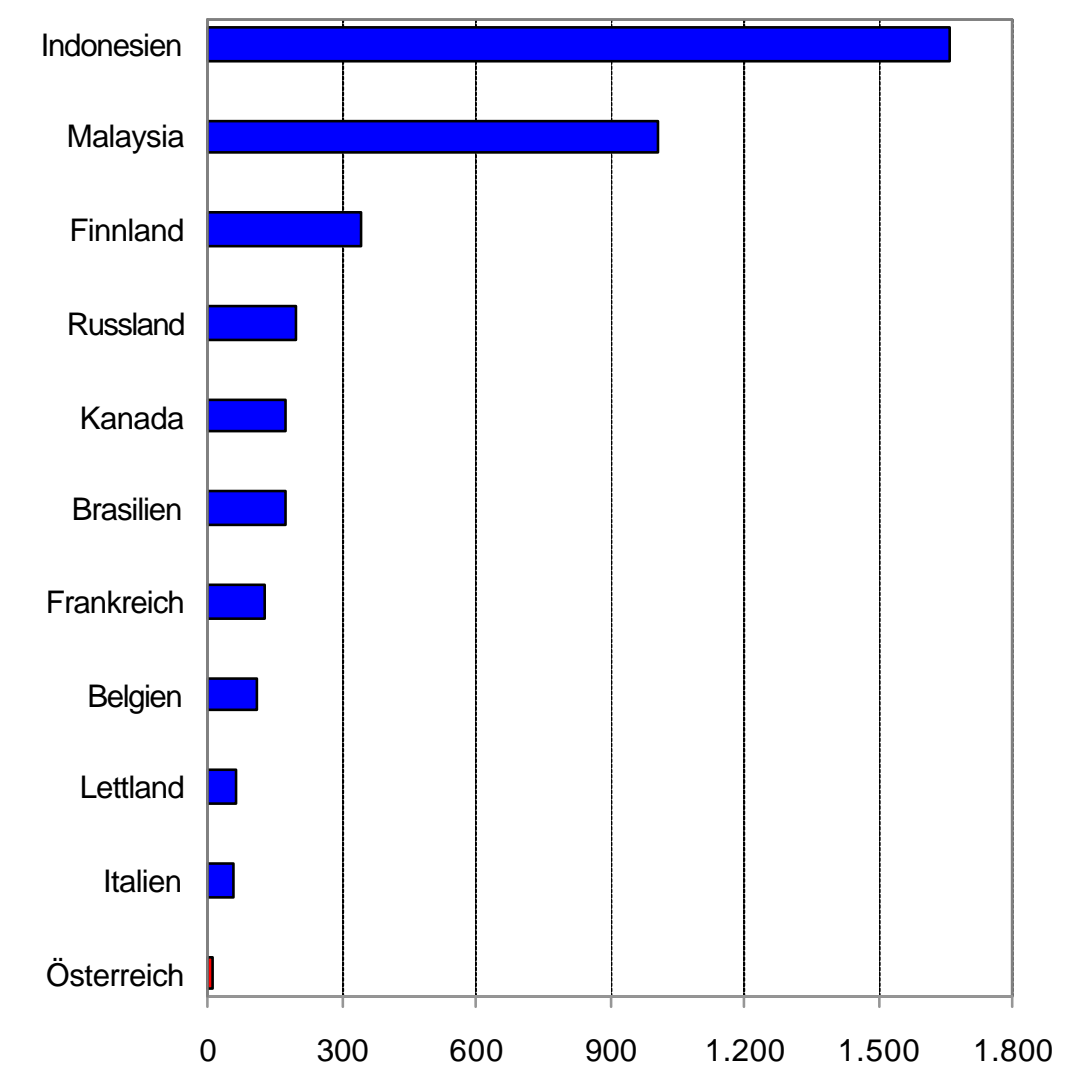


Abbildung 28: Dynamik der Exporte von anderem Sperrholz oder Furnier

\begin{tabular}{|c|c|c|c|}
\hline SITC 63439 & Exportwert & & RCA-Wert \\
\hline & \multicolumn{2}{|c|}{ Durchschnittliches jährl. Wachstum in \% } & \\
\hline & $1990 / 1997$ & $1997 / 2000$ & 2000 \\
\hline Indonesien & $+22,8$ & $-4,7$ & $+4,646$ \\
\hline Kanada & $+14,4$ & $+1,6$ & $+1,346$ \\
\hline Brasilien & $+30,6$ & $+48,2$ & $+6,427$ \\
\hline Finnland & $+24,1$ & $+6,1$ & $+4,528$ \\
\hline USA & $+3,6$ & $-19,0$ & $+0,860$ \\
\hline China & & $+17,3$ & $-0,692$ \\
\hline Chile & $+37,4$ & $+142,4$ & $+3,593$ \\
\hline Russland & . & $+11,4$ & $+4,070$ \\
\hline Frankreich & $+7,1$ & $+6,1$ & $-0,330$ \\
\hline Neuseeland & $+48,2$ & $-0,5$ & $+2,506$ \\
\hline Österreich & $+10,1$ & $+77,7$ & $-0,994$ \\
\hline
\end{tabular}

Quelle: UNO (WIFO-Datenbank)
Exportwert anderes Sperrholz oder Furnier In Mio. €

2000

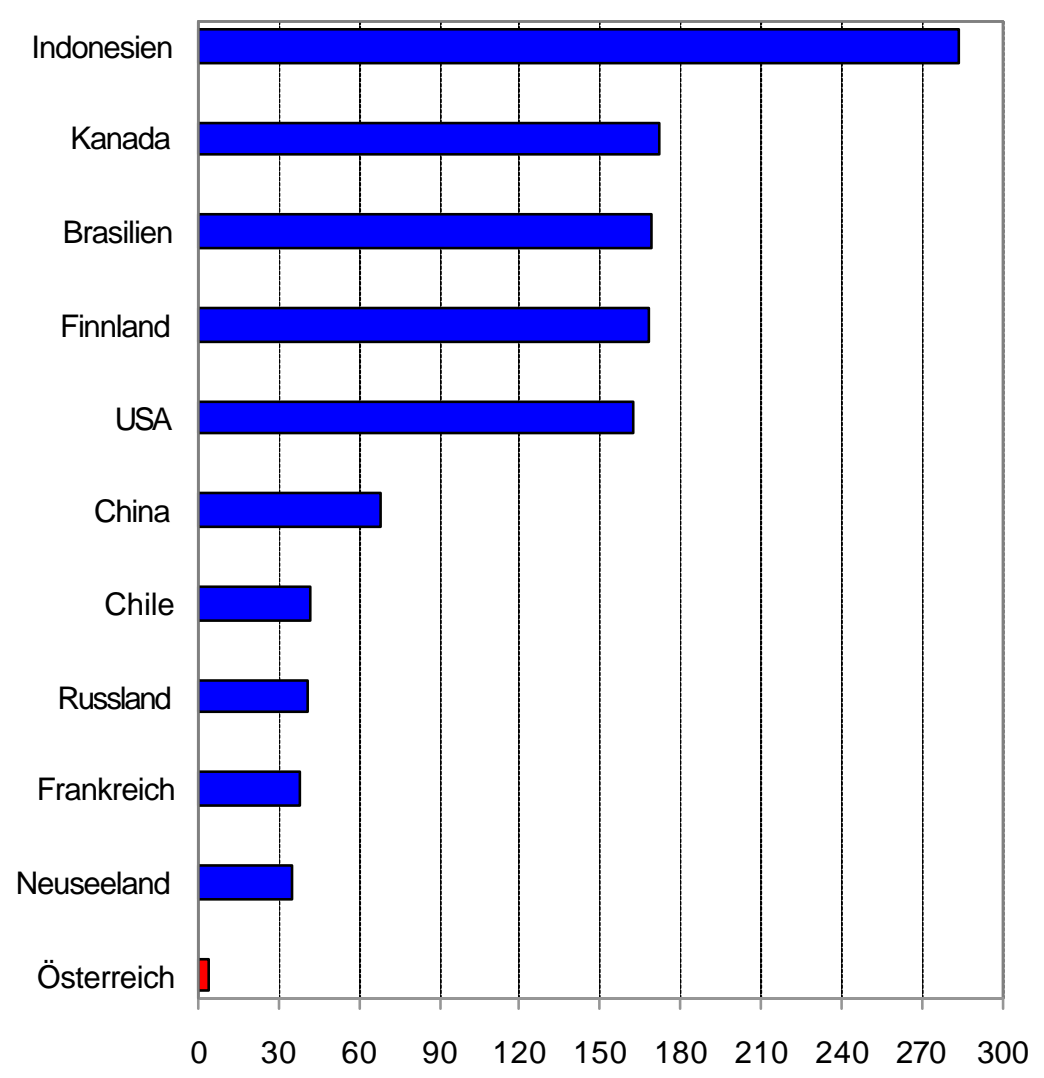


Abbildung 29: Dynamik der Exporte von and. Sperrholz aus Nadelholz

\begin{tabular}{|c|c|c|c|}
\hline SITC 63441 & Exportwert & & RCA-Wert \\
\hline & \multicolumn{2}{|c|}{ Durchschnittliches jährl. Wachstum in \% } & \\
\hline & $1990 / 1997$ & $1997 / 2000$ & 2000 \\
\hline Malaysia & $+57,3$ & $-8,7$ & $+3,139$ \\
\hline China & . & $+28,1$ & $+0,154$ \\
\hline Indonesien & $+11,0$ & $-25,4$ & $+5,678$ \\
\hline Deutschland & $-1,7$ & $+12,4$ & $-0,185$ \\
\hline Südkorea & $+1,1$ & $+22,9$ & $+0,606$ \\
\hline Italien & $+0,1$ & $+9,6$ & $+0,586$ \\
\hline Belgien & $-3,3$ & $+5,2$ & $+1,241$ \\
\hline Tschechien & . & $+31,3$ & $+1,822$ \\
\hline Kanada & $+29,2$ & $+14,0$ & $+0,556$ \\
\hline Brasilien & $+14,7$ & $-7,5$ & $+5,607$ \\
\hline Österreich & $+63,3$ & $-0,7$ & $-0,152$ \\
\hline
\end{tabular}

Quelle: UNO (WIFO-Datenbank)
Exportwert anderes Sperrholz aus Nadelholz In Mio. €

2000

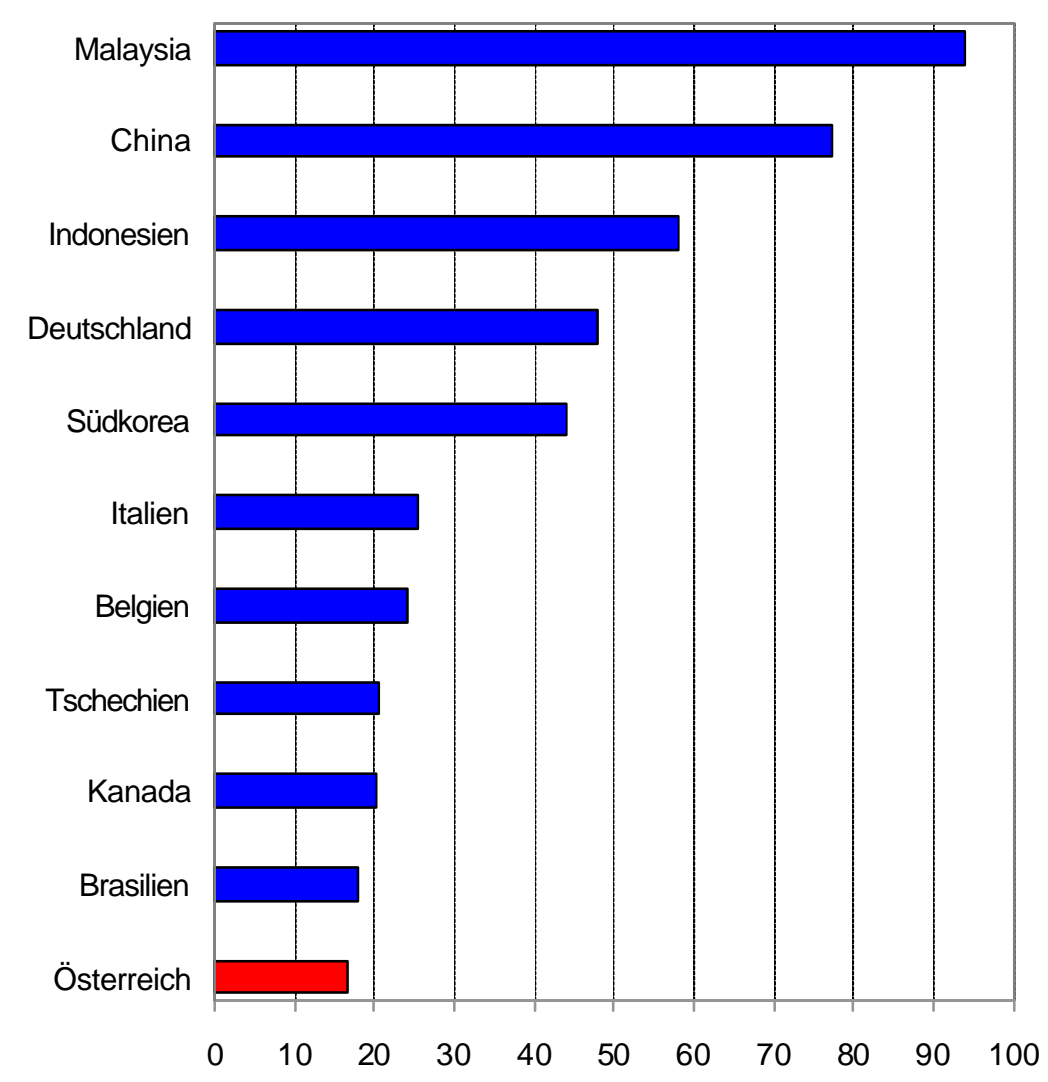


Abbildung 30: Dynamik der Exporte von anderem Sperrholz u.ä. a.n.g.

\begin{tabular}{|c|c|c|c|}
\hline SITC 63449 & Exportwert & & RCA-Wert \\
\hline & \multicolumn{2}{|c|}{ Durchschnittliches jährl. Wachstum in \% } & \\
\hline & $1990 / 1997$ & $1997 / 2000$ & 2000 \\
\hline Indonesien & $+28,9$ & $+0,7$ & $+5,529$ \\
\hline Österreich & $+0,6$ & $+8,5$ & $+1,639$ \\
\hline Brasilien & $-3,7$ & $+48,8$ & $+5,476$ \\
\hline Kanada & $+28,8$ & $+116,5$ & $+1,876$ \\
\hline Finnland & $+9,2$ & $+16,1$ & $+4,654$ \\
\hline Italien & $-4,3$ & $+4,3$ & $+0,077$ \\
\hline China & . & $-6,0$ & $-1,771$ \\
\hline Neuseeland & $+32,3$ & $+2,8$ & $+2,735$ \\
\hline Deutschland & $-0,0$ & $+4,9$ & $-0,326$ \\
\hline Polen & . & $+7,7$ & $+1,929$ \\
\hline
\end{tabular}

Quelle: UNO (WIFO-Datenbank)
Exportwert anderes Sperrholz u.ä. a.n.g. In Mio. € 2000

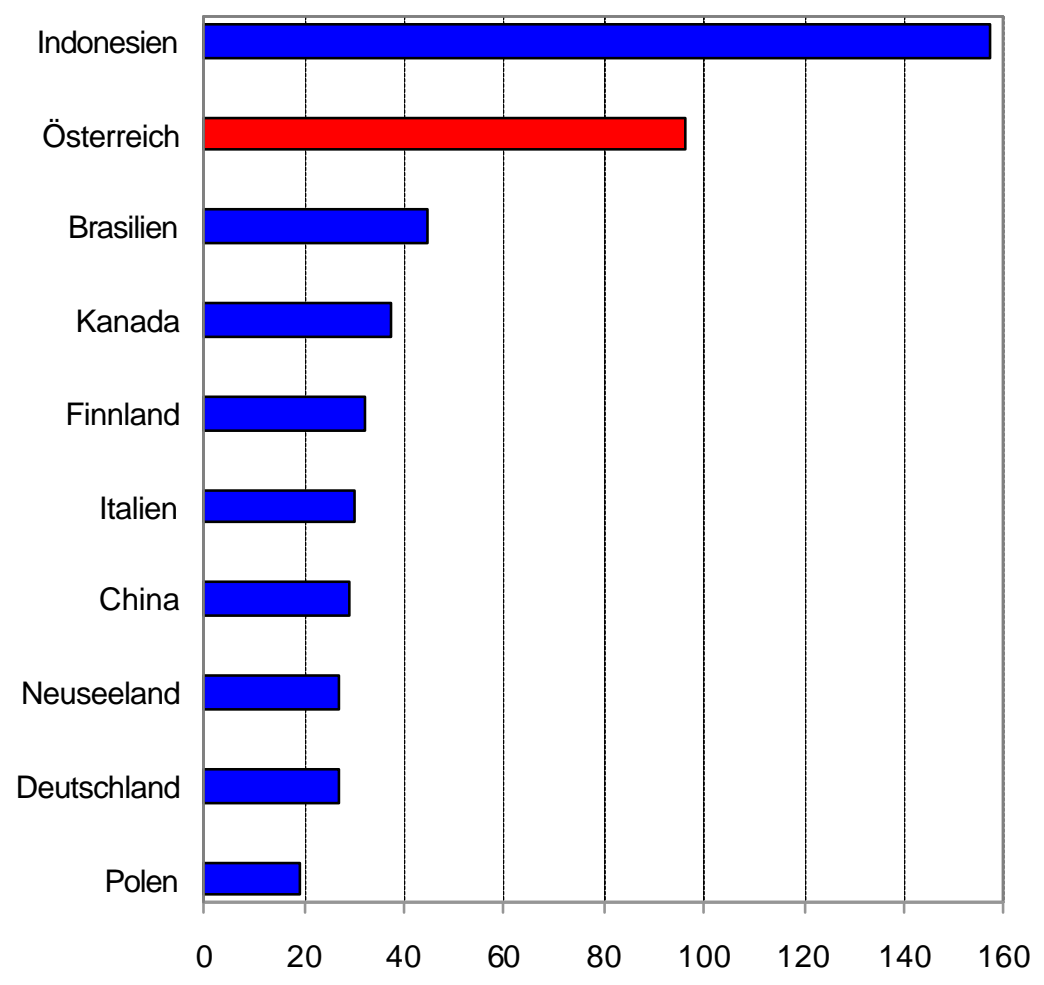


Abbildung 31: Dynamik der Exporte von Faserplatten > 0,8 g/cm3

\begin{tabular}{llll}
\hline SITC 63451 & Exportwert & RCA-Wert \\
\hline & Durchschnittliches jährl. Wachstum in $\%$ & \\
& $1990 / 1997$ & $1997 / 2000$ & 2000 \\
Deutschland & $+33,0$ & $+47,5$ & $+2,027$ \\
Österreich & $+17,2$ & $+25,2$ & $+1,187$ \\
USA & $+17,9$ & $+0,8$ & $-0,435$ \\
Frankreich & $+13,5$ & $+24,9$ & $+0,007$ \\
Kanada & $+19,3$ & $+25,4$ & $+0,043$ \\
Belgien & $+19,5$ & $+41,2$ & $-0,073$ \\
Brasilien & $+1,6$ & $-2,8$ & $+2,082$ \\
Polen & + & $+32,1$ & $+0,458$ \\
Spanien & $+16,1$ & $+12,8$ & $+1,210$ \\
Thailand & & &
\end{tabular}

Quelle: UNO (WIFO-Datenbank)

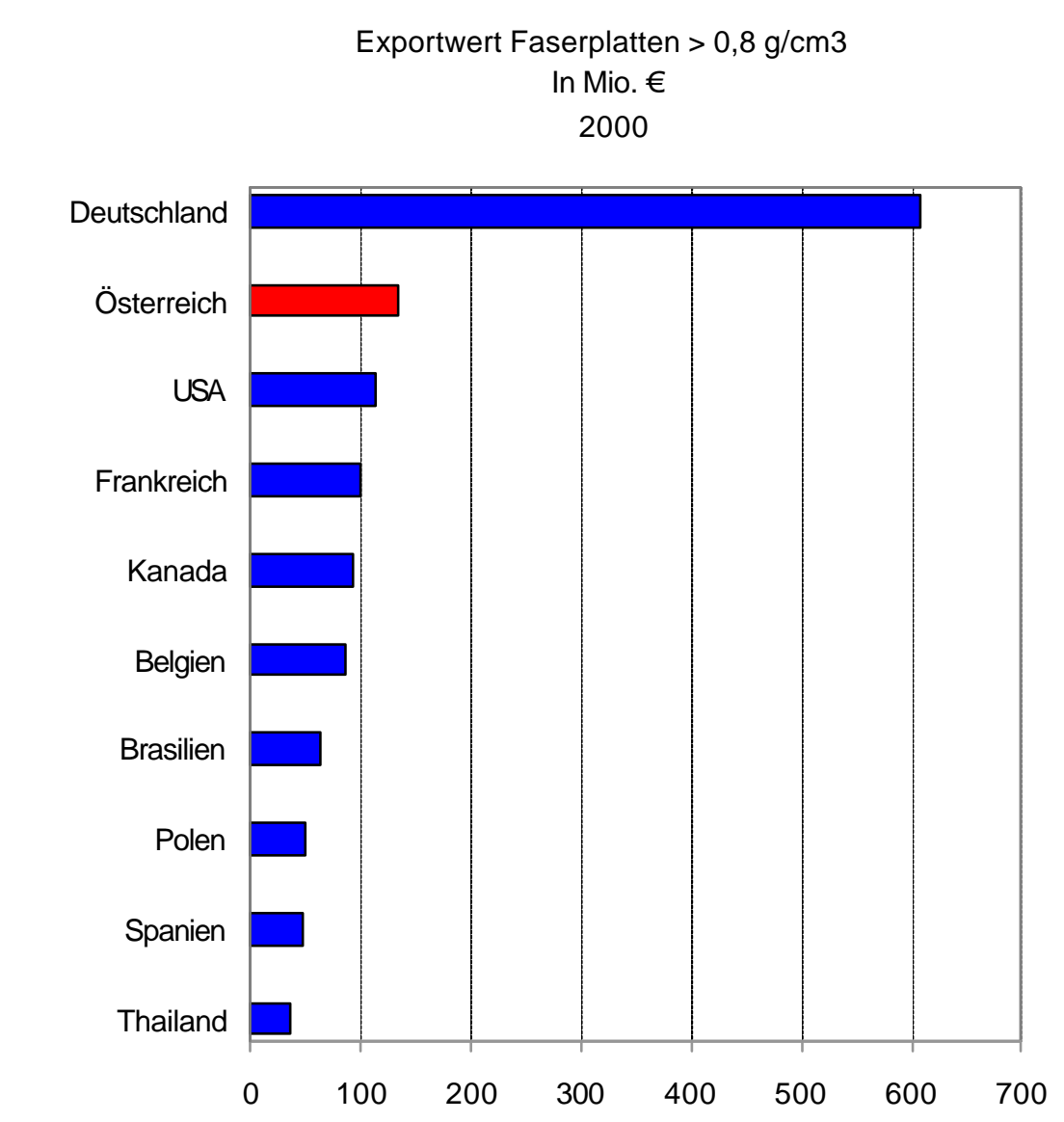


Abbildung 32: Dynamik der Exporte von Faserplatten 0,5 $0,8 \mathrm{~g} / \mathrm{cm} 3$

\begin{tabular}{|c|c|c|c|}
\hline SITC 63452 & Exportwert & & RCA-Wert \\
\hline & \multicolumn{2}{|c|}{ Durchschnittliches jährl. Wachstum in \% } & \\
\hline & $1990 / 1997$ & $1997 / 2000$ & 2000 \\
\hline Malaysia & . & $+37,7$ & $+3,966$ \\
\hline Kanada & $+19,3$ & $+42,7$ & $+1,350$ \\
\hline Deutschland & $+24,3$ & $+7,6$ & $+0,663$ \\
\hline Neuseeland & $+7,4$ & $+14,6$ & $+4,509$ \\
\hline Italien & $+14,9$ & $-2,0$ & $+0,883$ \\
\hline Frankreich & $+24,2$ & $+16,8$ & $+0,443$ \\
\hline Schweiz & 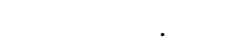 & . & $+1,772$ \\
\hline Spanien & $+4,9$ & $+18,6$ & $+0,287$ \\
\hline Portugal & $+18,8$ & $+0,2$ & $+2,522$ \\
\hline Irland & $+9,7$ & $-3,1$ & $+1,276$ \\
\hline Österreich & . & $+14,1$ & $+0,831$ \\
\hline
\end{tabular}

Quelle: UNO (WIFO-Datenbank)
Exportwert Faserplatten $0,5-0,8 \mathrm{~g} / \mathrm{cm} 3$ In Mio. €

2000

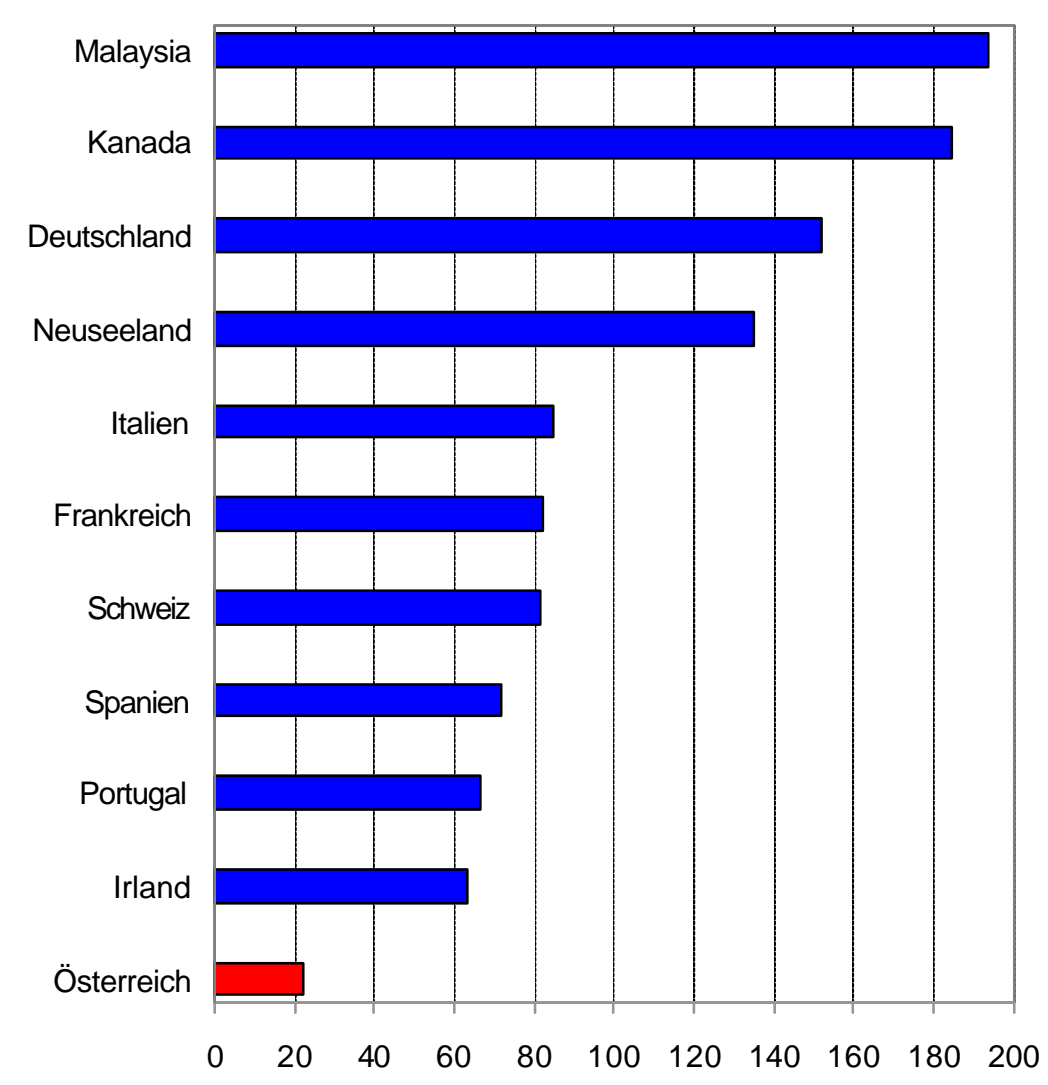


Abbildung 33: Dynamik der Exporte von Faserplatten 0,35 $0,5 \mathrm{~g} / \mathrm{cm} 3$

\begin{tabular}{|c|c|c|c|}
\hline SITC 63453 & Exportwert & & RCA-Wert \\
\hline & \multicolumn{2}{|c|}{ Durchschnittliches jährl. Wachstum in \% } & \\
\hline & $1990 / 1997$ & $1997 / 2000$ & 2000 \\
\hline Chile & . & $+45,4$ & $+0,000$ \\
\hline Luxemburg & $\cdot$ & $\cdot$ & $+7,442$ \\
\hline Japan & $-10,7$ & $+0,2$ & $+1,783$ \\
\hline USA & $-6,1$ & $+3,1$ & $-1,588$ \\
\hline Österreich & $+3,7$ & $+145,2$ & $+0,421$ \\
\hline Schweden & $+38,0$ & $-7,4$ & $+0,303$ \\
\hline Belgien & . & $+159,8$ & $+1,719$ \\
\hline Spanien & & & $-0,027$ \\
\hline Norwegen & $+22,8$ & $-9,7$ & $+0,154$ \\
\hline Kanada & & $+7,1$ & $-1,768$ \\
\hline Belgien-Lux. & $+112,6$ & . & . \\
\hline
\end{tabular}

Quelle: UNO (WIFO-Datenbank)
Exportwert Faserplatten 0,35 - 0,5 g/cm3 In Mio. €

2000

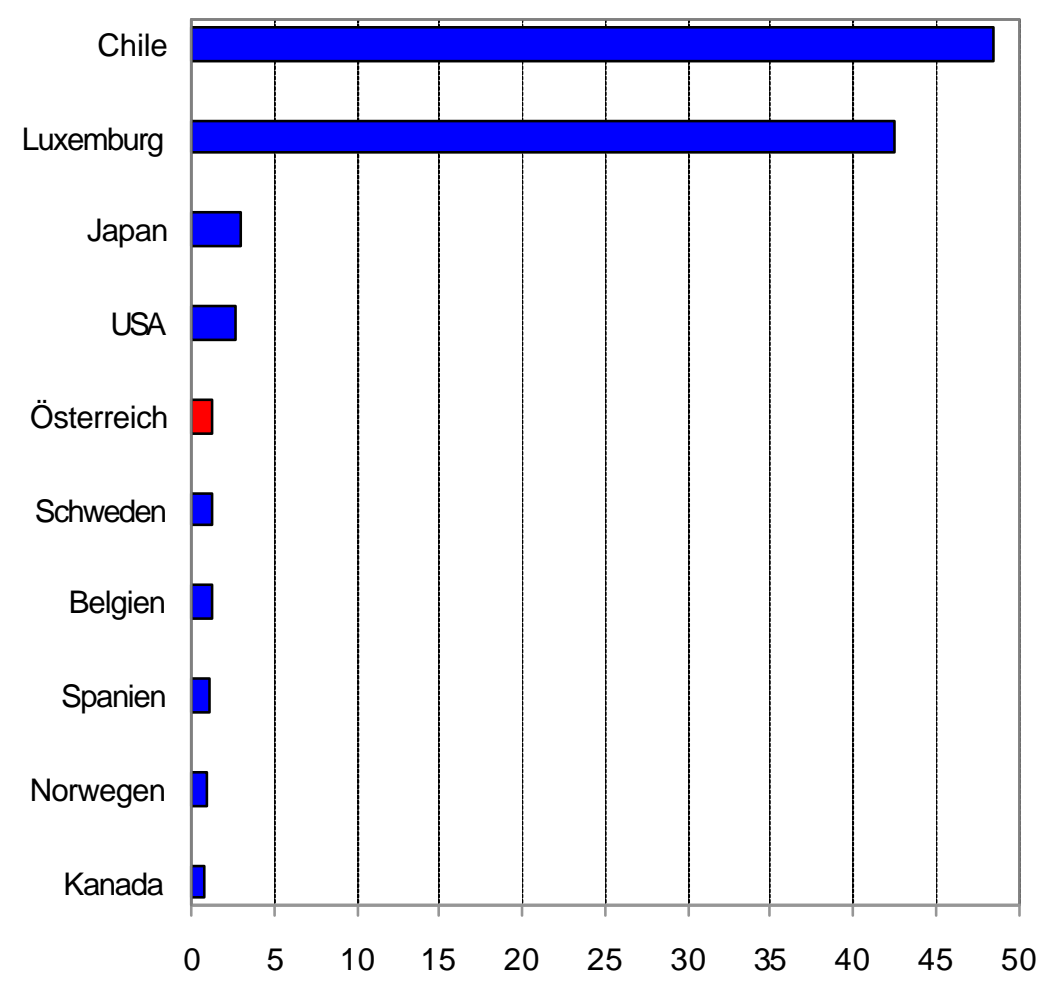


Abbildung 34: Dynamik der Exporte von Kisten, Trommeln

$$
\text { u.ä. }
$$

\begin{tabular}{|c|c|c|c|}
\hline SITC 63511 & Exportwert & & RCA-Wert \\
\hline & \multicolumn{2}{|c|}{ Durchschnittliches jährl. Wachstum in \% } & \\
\hline & 1990/1997 & $1997 / 2000$ & 2000 \\
\hline USA & $+13,4$ & $+11,1$ & $+0,081$ \\
\hline Deutschland & $+6,8$ & $+9,0$ & $-0,540$ \\
\hline Spanien & $+7,5$ & $+3,3$ & $+1,863$ \\
\hline Kanada & $+35,8$ & $+2,3$ & $+0,808$ \\
\hline Frankreich & $+1,7$ & $+1,8$ & $-0,201$ \\
\hline China & . & $+24,8$ & $+3,037$ \\
\hline Tschechien & . & $+12,0$ & $+2,380$ \\
\hline Schweden & $-2,0$ & $+16,2$ & $+1,707$ \\
\hline Mexiko & $+36,8$ & $+15,6$ & $-0,022$ \\
\hline Indonesien & $+11,2$ & $+57,2$ & $+3,004$ \\
\hline Österreich & $+12,3$ & $+3,2$ & $-0,459$ \\
\hline
\end{tabular}

Quelle: UNO (WIFO-Datenbank)

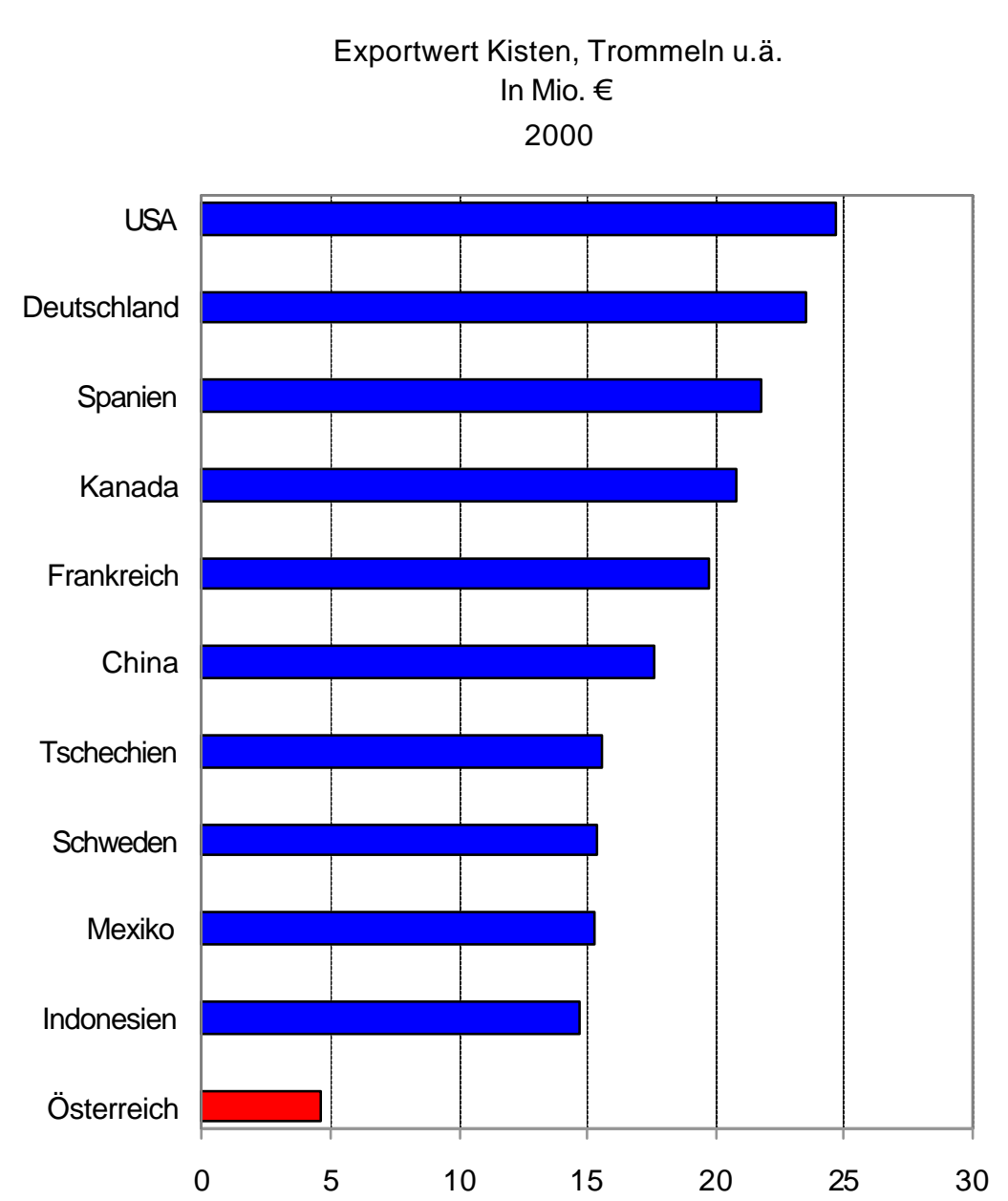


Abbildung 35: Dynamik der Exporte von Flachpaletten u.dgl.

\begin{tabular}{|c|c|c|c|}
\hline SITC 63512 & Exportwert & & RCA-Wert \\
\hline & \multicolumn{2}{|c|}{ Durchschnittliches jährl. Wachstum in \% } & \\
\hline & $1990 / 1997$ & $1997 / 2000$ & 2000 \\
\hline Polen & . & $+12,5$ & $+2,503$ \\
\hline Kanada & $+43,0$ & $+53,8$ & $+1,739$ \\
\hline Belgien & $+7,2$ & $+28,1$ & $+0,262$ \\
\hline Großbritannien & $+21,3$ & $+15,4$ & $-0,160$ \\
\hline Deutschland & $+12,3$ & $+12,4$ & $-0,822$ \\
\hline Niederlande & $+1,7$ & $+18,5$ & $+0,236$ \\
\hline Frankreich & $+9,0$ & $+16,3$ & $-0,530$ \\
\hline Tschechien & & $+12,6$ & $+1,852$ \\
\hline Mexiko & $+68,8$ & $+15,2$ & $+0,885$ \\
\hline Italien & $+28,3$ & $+10,9$ & $-0,499$ \\
\hline Österreich & $+0,7$ & $+22,0$ & $-0,715$ \\
\hline
\end{tabular}

Quelle: UNO (WIFO-Datenbank)

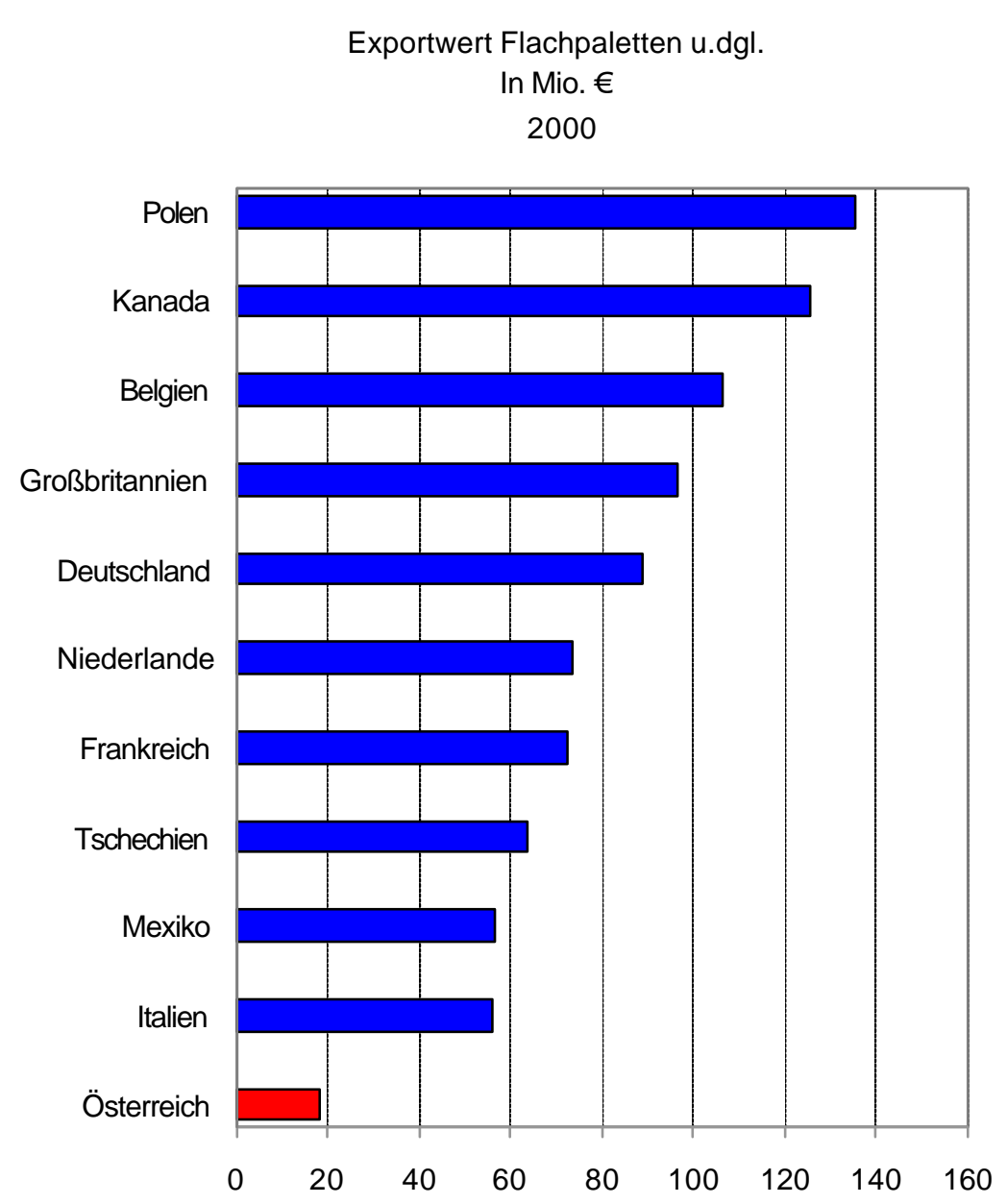


Abbildung 36: Dynamik der Exporte von Fässern, Trögen u.dgl.

\begin{tabular}{|c|c|c|c|}
\hline SITC 6352 & Exportwert & & RCA-Wert \\
\hline & \multicolumn{2}{|c|}{ Durchschnittliches jährl. Wachstum in \% } & \\
\hline & $1990 / 1997$ & $1997 / 2000$ & 2000 \\
\hline Frankreich & $+16,3$ & $+24,5$ & $+2,350$ \\
\hline USA & $+9,5$ & $+34,5$ & $+0,459$ \\
\hline Spanien & $-7,0$ & $+31,7$ & $-0,375$ \\
\hline Ungarn & . & $+51,0$ & $+2,328$ \\
\hline Portugal & $+2,1$ & $+26,4$ & $+1,630$ \\
\hline China & . & $+2,4$ & $+1,346$ \\
\hline Österreich & $+35,6$ & $+27,0$ & $-0,360$ \\
\hline Chile & . & $+50,5$ & $-1,714$ \\
\hline Deutschland & $+4,8$ & $-17,4$ & $-0,371$ \\
\hline Rumänien & . & $+164,1$ & $+3,681$ \\
\hline
\end{tabular}

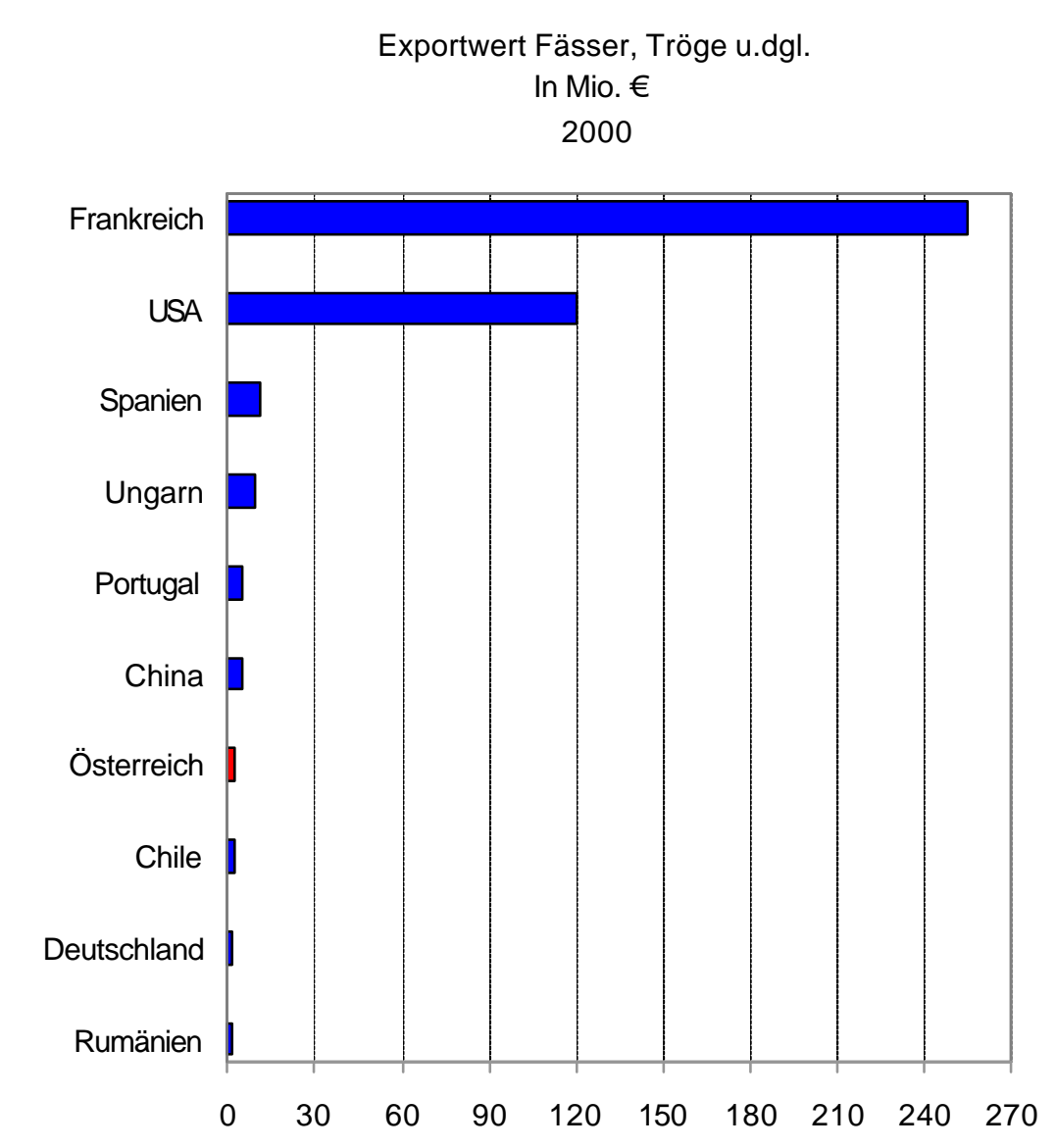


Abbildung 37: Dynamik der Exporte von Holzfenstern u.dgl.

\begin{tabular}{|c|c|c|c|}
\hline SITC 63531 & Exportwert & & RCA-Wert \\
\hline & \multicolumn{2}{|c|}{ Durchschnittliches jährl. Wachstum in \% } & \\
\hline & $1990 / 1997$ & $1997 / 2000$ & 2000 \\
\hline Dänemark & $+6,3$ & $+7,9$ & $+2,905$ \\
\hline Kanada & $+34,5$ & $+25,6$ & $+1,370$ \\
\hline USA & $+7,7$ & $+9,1$ & $-0,103$ \\
\hline Polen & . & $+13,4$ & $+1,555$ \\
\hline Slowenien & . & $-5,1$ & $+3,146$ \\
\hline Großbritannien & $+26,0$ & $+10,9$ & $-0,206$ \\
\hline Deutschland & $-1,5$ & $-0,9$ & $-1,452$ \\
\hline Ungarn & . & $+13,7$ & $+2,978$ \\
\hline Frankreich & $+13,9$ & $-7,3$ & $-0,341$ \\
\hline Norwegen & $-5,9$ & $+2,7$ & $+0,592$ \\
\hline Österreich & $+5,5$ & $-0,4$ & $-0,026$ \\
\hline
\end{tabular}

Quelle: UNO (WIFO-Datenbank)

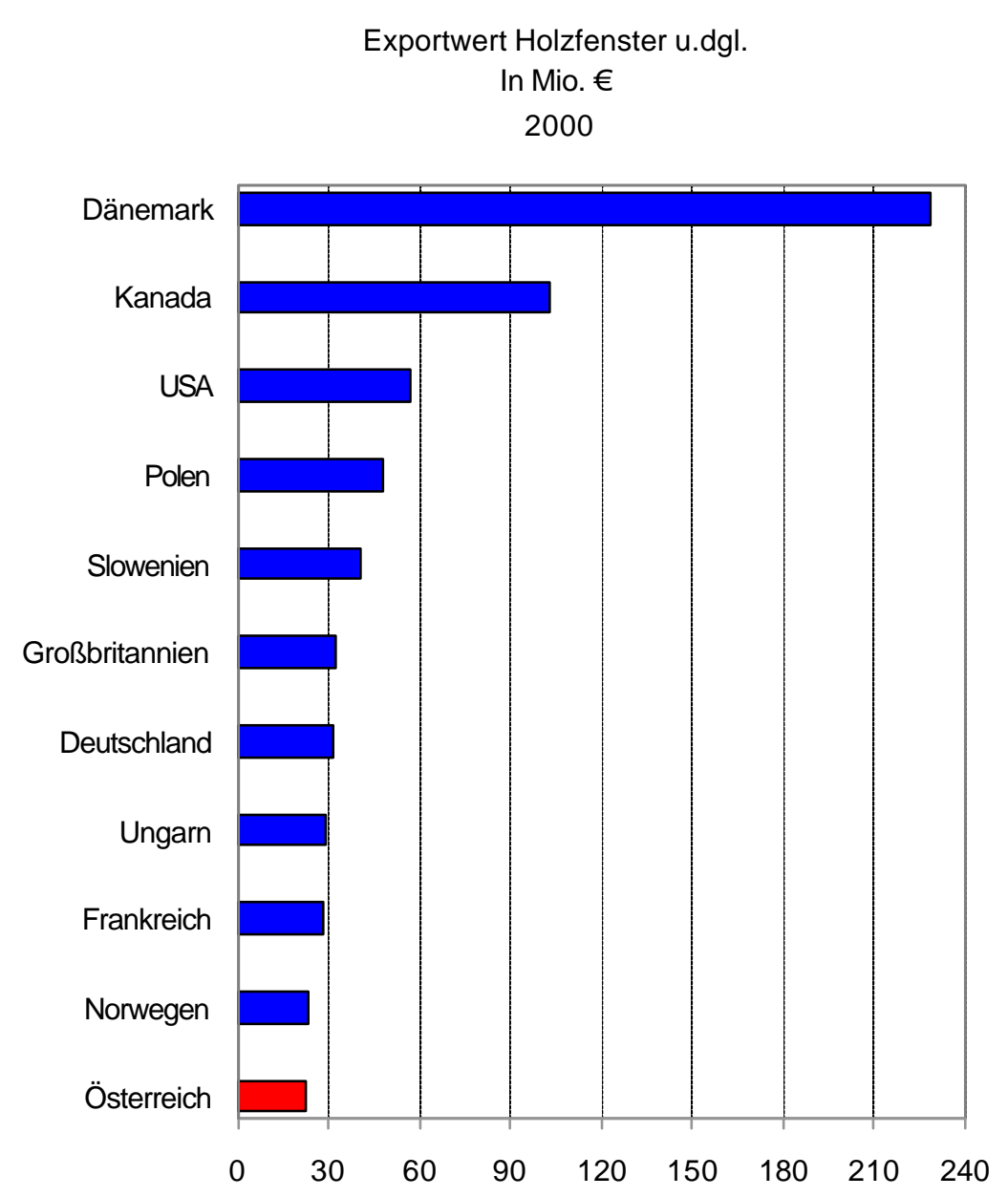


Abbildung 38: Dynamik der Exporte von Holztüren u.dgl.

\begin{tabular}{|c|c|c|c|}
\hline SITC 63532 & Exportwert & & RCA-Wert \\
\hline & \multicolumn{2}{|c|}{ Durchschnittliches jährl. Wachstum in \% } & \\
\hline & $1990 / 1997$ & $1997 / 2000$ & 2000 \\
\hline Kanada & $+30,3$ & $+33,6$ & $+1,235$ \\
\hline Indonesien & $+23,8$ & $+2,6$ & $+7,237$ \\
\hline China & . & $+20,9$ & $+4,097$ \\
\hline USA & $+12,8$ & $+0,2$ & $-0,935$ \\
\hline Brasilien & $+17,5$ & $+19,1$ & $+6,754$ \\
\hline Dänemark & $+14,6$ & $+8,1$ & $+0,469$ \\
\hline Malaysia & $+13,5$ & $+4,8$ & $+5,344$ \\
\hline Deutschland & $+1,8$ & $+5,4$ & $-0,697$ \\
\hline Schweden & $+5,7$ & $+1,4$ & $+1,401$ \\
\hline Spanien & $+21,0$ & $+3,0$ & $+2,556$ \\
\hline Österreich & $+3,9$ & $-0,1$ & $-0,142$ \\
\hline
\end{tabular}

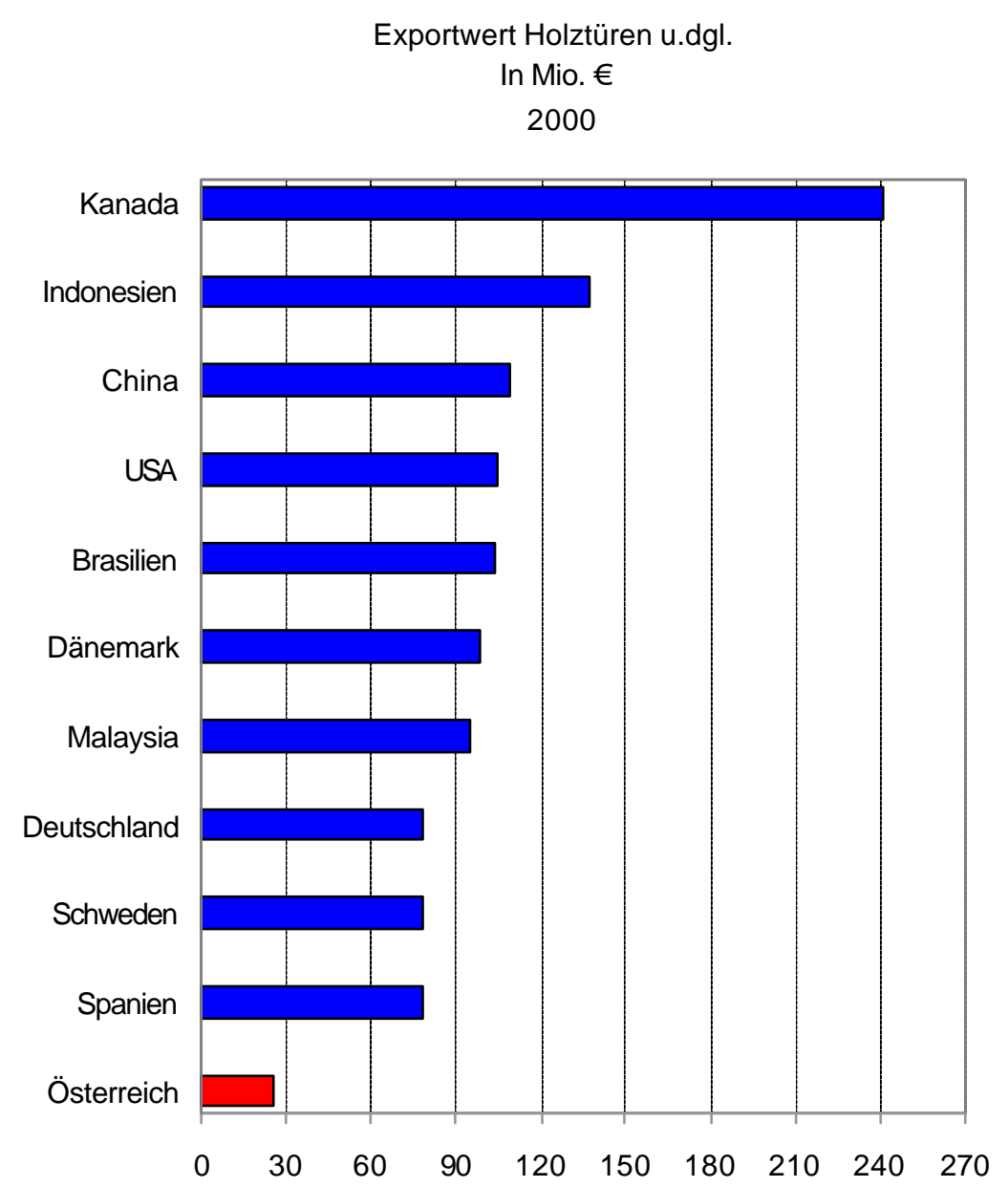


Abbildung 39: Dynamik der Exporte von anderen

Bautischlerarbeiten (darunter

Konstruktionsholz)

\begin{tabular}{|c|c|c|c|}
\hline SITC 63539 & Exportwert & & RCA-Wert \\
\hline & \multicolumn{2}{|c|}{ Durchschnittliches jährl. Wachstum in \% } & \\
\hline & 1990/1997 & $1997 / 2000$ & 2000 \\
\hline Kanada & $+44,0$ & $+24,0$ & $+2,088$ \\
\hline Indonesien & $+14,3$ & $+19,7$ & $+6,656$ \\
\hline Österreich & $+9,1$ & $+22,8$ & $+0,916$ \\
\hline Deutschland & $+4,9$ & $+6,5$ & $-0,836$ \\
\hline Belgien & $+15,9$ & $+27,2$ & $+1,022$ \\
\hline Schweden & $+13,8$ & $-10,1$ & $+1,399$ \\
\hline USA & $+13,6$ & $-1,1$ & $-0,920$ \\
\hline China & & $+17,8$ & $+2,968$ \\
\hline Finnland & $+27,2$ & $+8,6$ & $+1,935$ \\
\hline Philippinen & & $+80,1$ & $+4,364$ \\
\hline
\end{tabular}

Quelle: UNO (WIFO-Datenbank)
Exportwert andere Bautischlerarbeiten

In Mio. €

2000

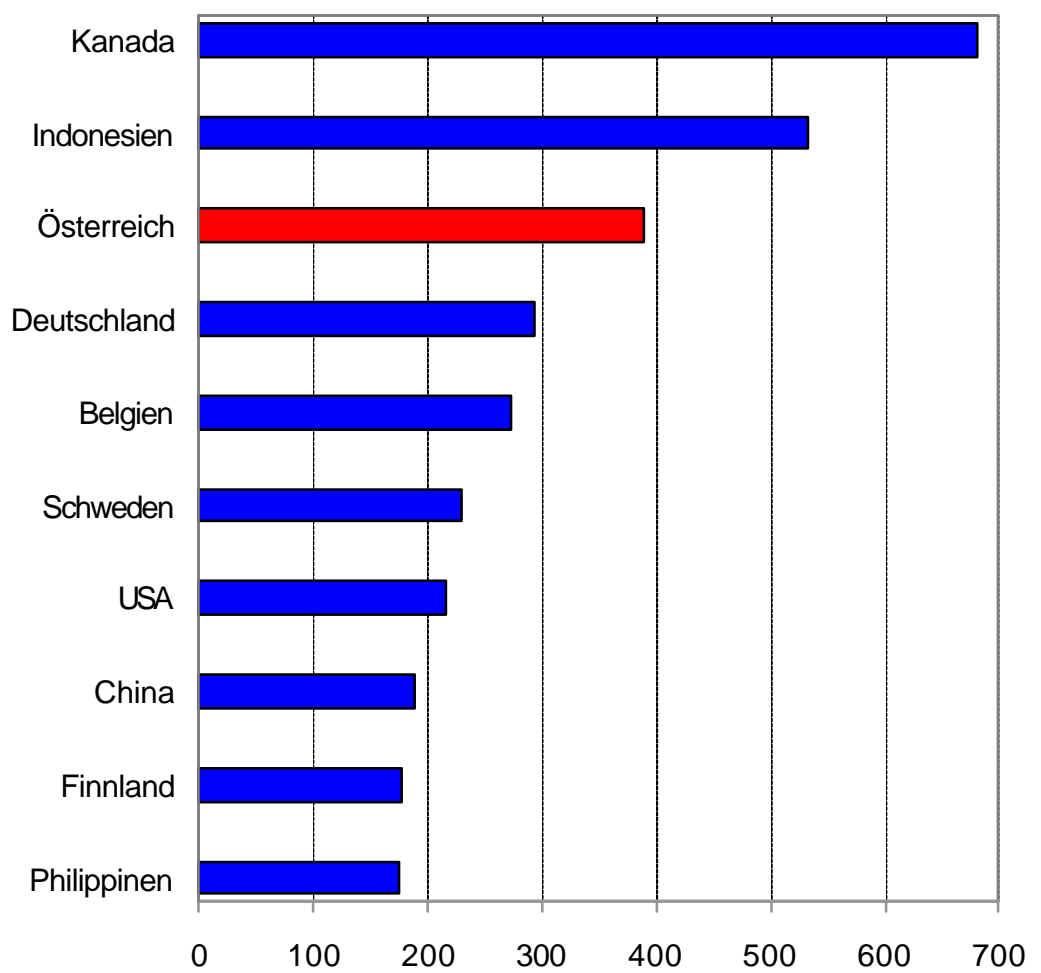


Abbildung 40: Dynamik der Exporte von vorgefertigten

\section{Häusern}

\begin{tabular}{|c|c|c|c|}
\hline SITC 811 & Exportwert & & RCA-Wert \\
\hline & \multicolumn{2}{|c|}{ Durchschnittliches jährl. Wachstum in \% } & \\
\hline & 1990/1997 & $1997 / 2000$ & 2000 \\
\hline USA & $+17,2$ & $-4,2$ & $+0,693$ \\
\hline Kanada & $+22,0$ & $+12,3$ & $+1,281$ \\
\hline Belgien & $+8,1$ & $+3,2$ & $+0,972$ \\
\hline Schweden & $+11,2$ & $+8,5$ & $+2,136$ \\
\hline Frankreich & $+5,2$ & $+8,6$ & $-0,007$ \\
\hline Finnland & $+1,0$ & $+5,2$ & $+2,678$ \\
\hline Niederlande & $+6,9$ & $-4,3$ & $+1,139$ \\
\hline Tschechien & . & $+16,3$ & $+2,521$ \\
\hline Großbritannien & $+10,4$ & $-3,1$ & $+1,142$ \\
\hline Spanien & $+34,8$ & $+27,1$ & $+1,428$ \\
\hline Österreich & $+9,2$ & $+6,5$ & $-0,198$ \\
\hline
\end{tabular}

Quelle: UNO (WIFO-Datenbank)

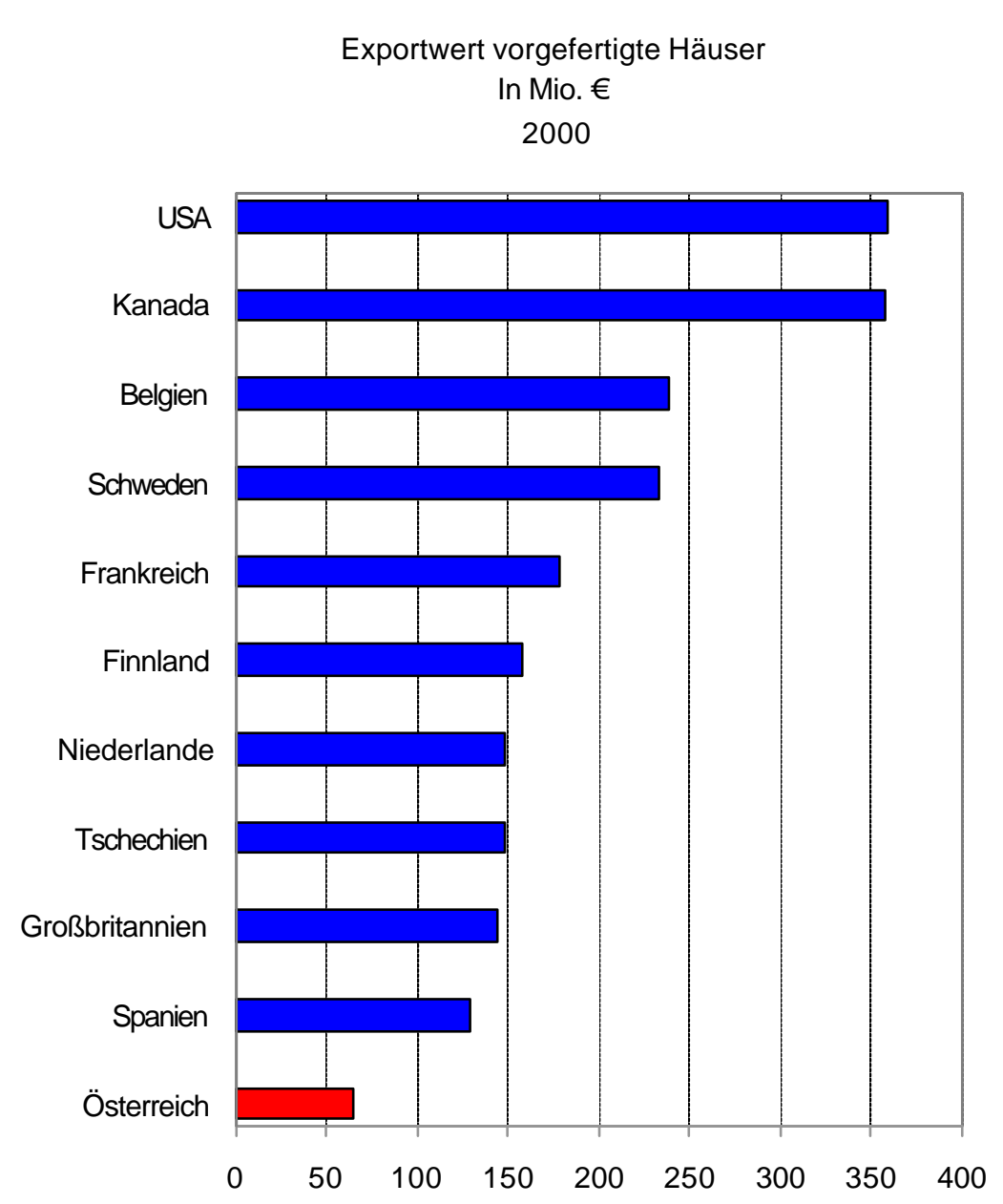


Abbildung 41: Dynamik der Exporte von Sitzen a.n.g. mit Holzgestell

\begin{tabular}{|c|c|c|c|}
\hline SITC 82116 & Exportwert & & RCA-Wert \\
\hline & \multicolumn{2}{|c|}{ Durchschnittliches jährl. Wachstum in \% } & \\
\hline & $1990 / 1997$ & $1997 / 2000$ & 2000 \\
\hline Italien & $+9,7$ & $+6,9$ & $+3,400$ \\
\hline Polen & . & $+14,1$ & $+4,034$ \\
\hline China & . & $+25,8$ & $+4,120$ \\
\hline Deutschland & $-4,5$ & $+11,6$ & $-1,041$ \\
\hline Belgien & $+1,7$ & $+11,1$ & $+0,127$ \\
\hline Kanada & $+17,2$ & $+31,3$ & $+0,129$ \\
\hline Malaysia & $+51,9$ & $+22,4$ & $+3,991$ \\
\hline Thailand & $+14,3$ & $+14,8$ & $+4,501$ \\
\hline USA & $+8,8$ & $+7,7$ & $-1,709$ \\
\hline Mexiko & $+84,9$ & $+26,7$ & $+2,103$ \\
\hline Österreich & $+1,2$ & $-2,7$ & $-0,983$ \\
\hline
\end{tabular}

Quelle: UNO (WIFO-Datenbank)

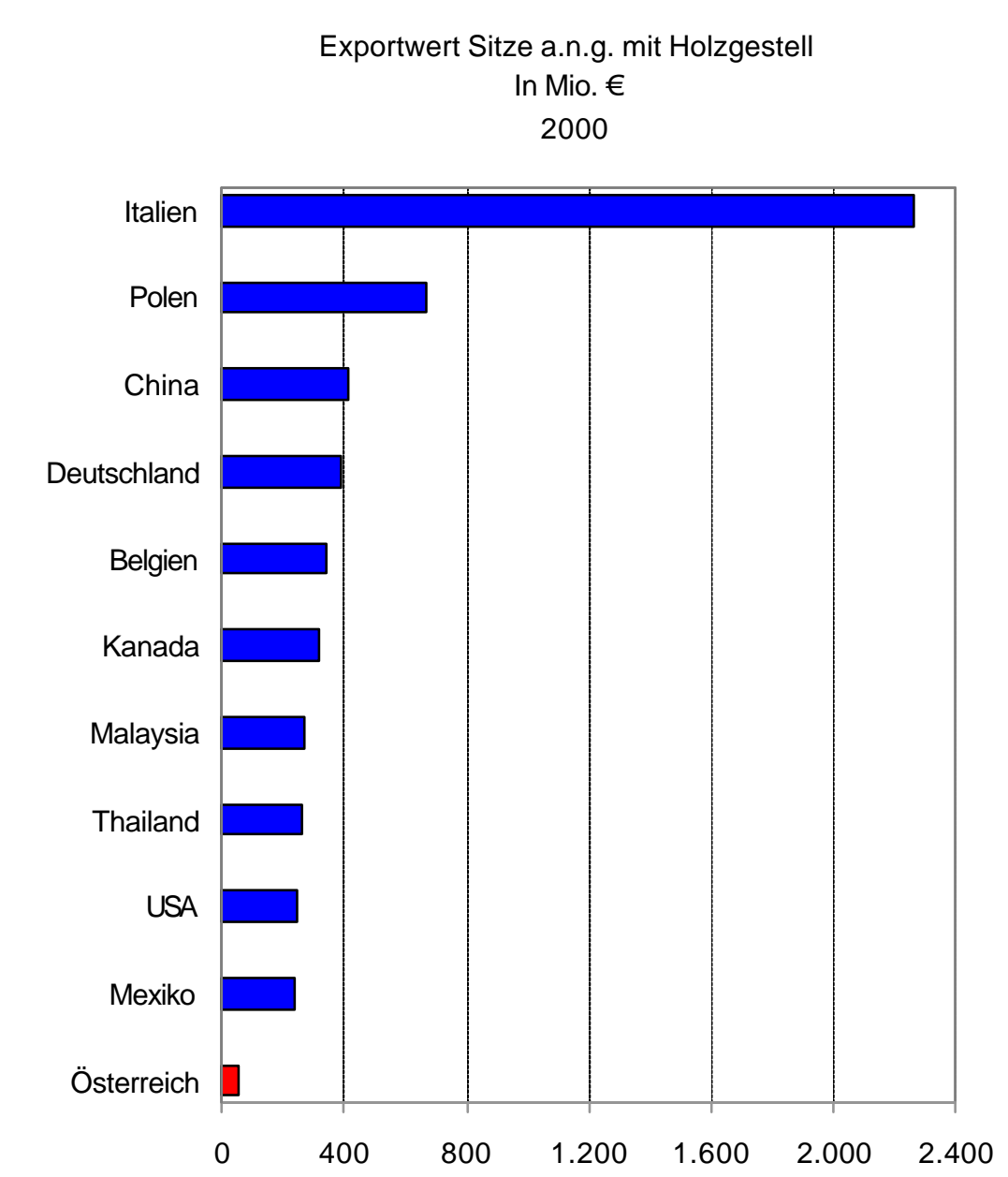


Abbildung 42: Dynamik der Exporte von Büromöbeln aus

\section{Holz}

\begin{tabular}{|c|c|c|c|}
\hline SITC 82151 & Exportwert & & RCA-Wert \\
\hline & \multicolumn{2}{|c|}{ Durchschnittliches jährl. Wachstum in \% } & \\
\hline & 1990/1997 & $1997 / 2000$ & 2000 \\
\hline Kanada & $+21,9$ & $+24,9$ & $+1,876$ \\
\hline Italien & $+9,1$ & $+1,3$ & $+2,552$ \\
\hline Deutschland & $-0,5$ & $+8,6$ & $+0,407$ \\
\hline Dänemark & $+9,0$ & $+18,0$ & $+1,366$ \\
\hline Schweden & $+10,3$ & $+1,3$ & $+1,999$ \\
\hline USA & $+12,2$ & $+12,8$ & $-1,404$ \\
\hline China & . & $+21,2$ & $+3,670$ \\
\hline Malaysia & $+63,7$ & $+19,3$ & $+3,992$ \\
\hline Polen & . & $+6,2$ & $+1,907$ \\
\hline Großbritannien & $+13,4$ & $+3,3$ & $-0,845$ \\
\hline Österreich & $+12,5$ & $+4,5$ & $+0,304$ \\
\hline
\end{tabular}

Quelle: UNO (WIFO-Datenbank)

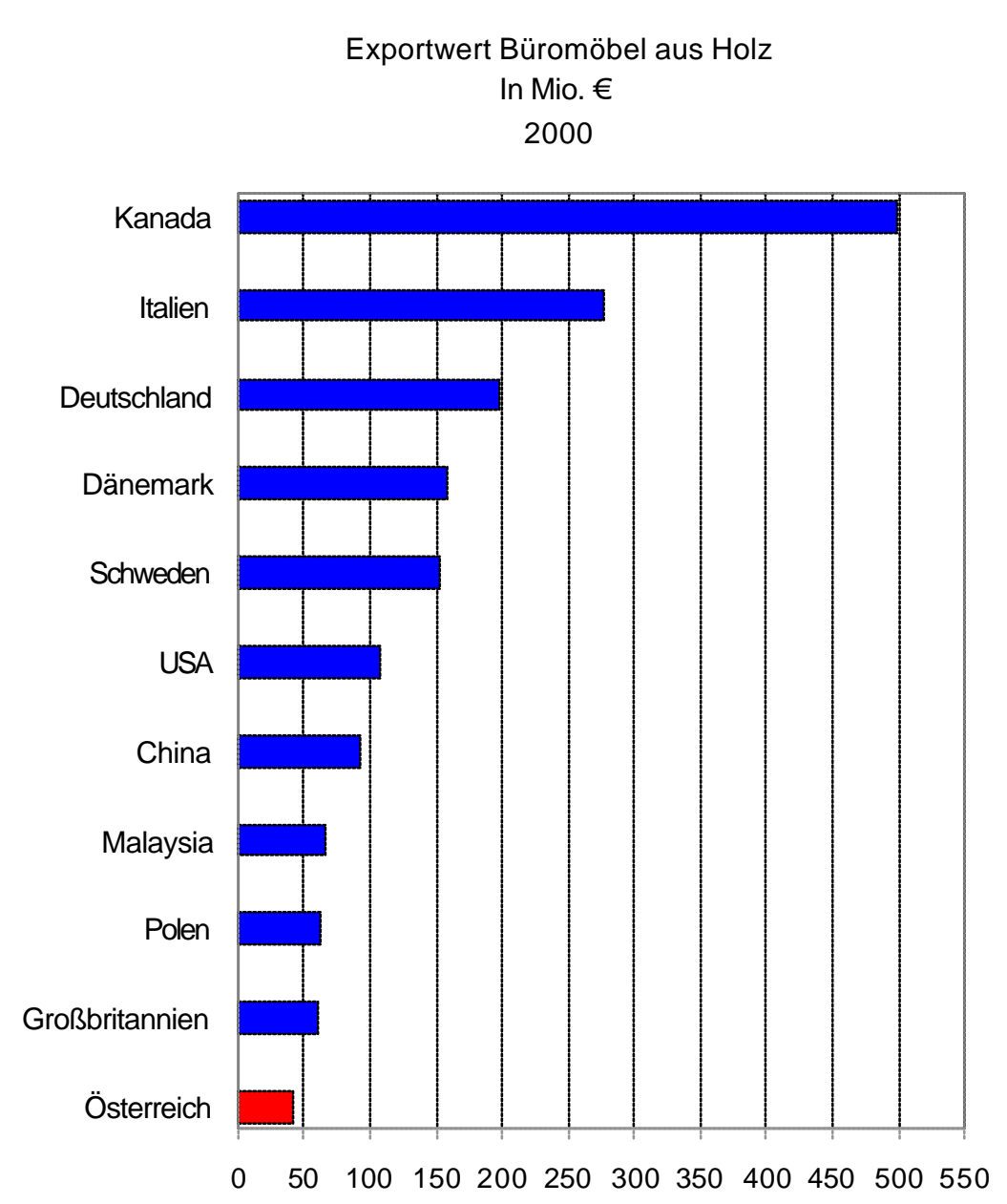


Abbildung 43: Dynamik der Exporte von Küchenmöbeln aus

\section{Holz}

\begin{tabular}{|c|c|c|c|}
\hline SITC 82153 & Exportwert & & RCA-Wert \\
\hline & \multicolumn{2}{|c|}{ Durchschnittliches jährl. Wachstum in \% } & \\
\hline & $1990 / 1997$ & $1997 / 2000$ & 2000 \\
\hline Deutschland & $+0,4$ & $+10,6$ & $+1,958$ \\
\hline Kanada & $+36,6$ & $+29,6$ & $+2,716$ \\
\hline Italien & $+21,3$ & $+8,4$ & $+2,660$ \\
\hline China & . & $+26,2$ & $+3,688$ \\
\hline Malaysia & $+44,1$ & $+9,4$ & $+4,347$ \\
\hline Frankreich & $+0,6$ & $+0,5$ & $-0,334$ \\
\hline Dänemark & $-0,9$ & $+18,9$ & $+1,856$ \\
\hline Spanien & $+33,1$ & $+15,3$ & $+1,177$ \\
\hline Österreich & $+20,4$ & $-2,8$ & $+0,010$ \\
\hline USA & $+11,6$ & $-0,1$ & $-1,956$ \\
\hline
\end{tabular}

Quelle: UNO (WIFO-Datenbank)

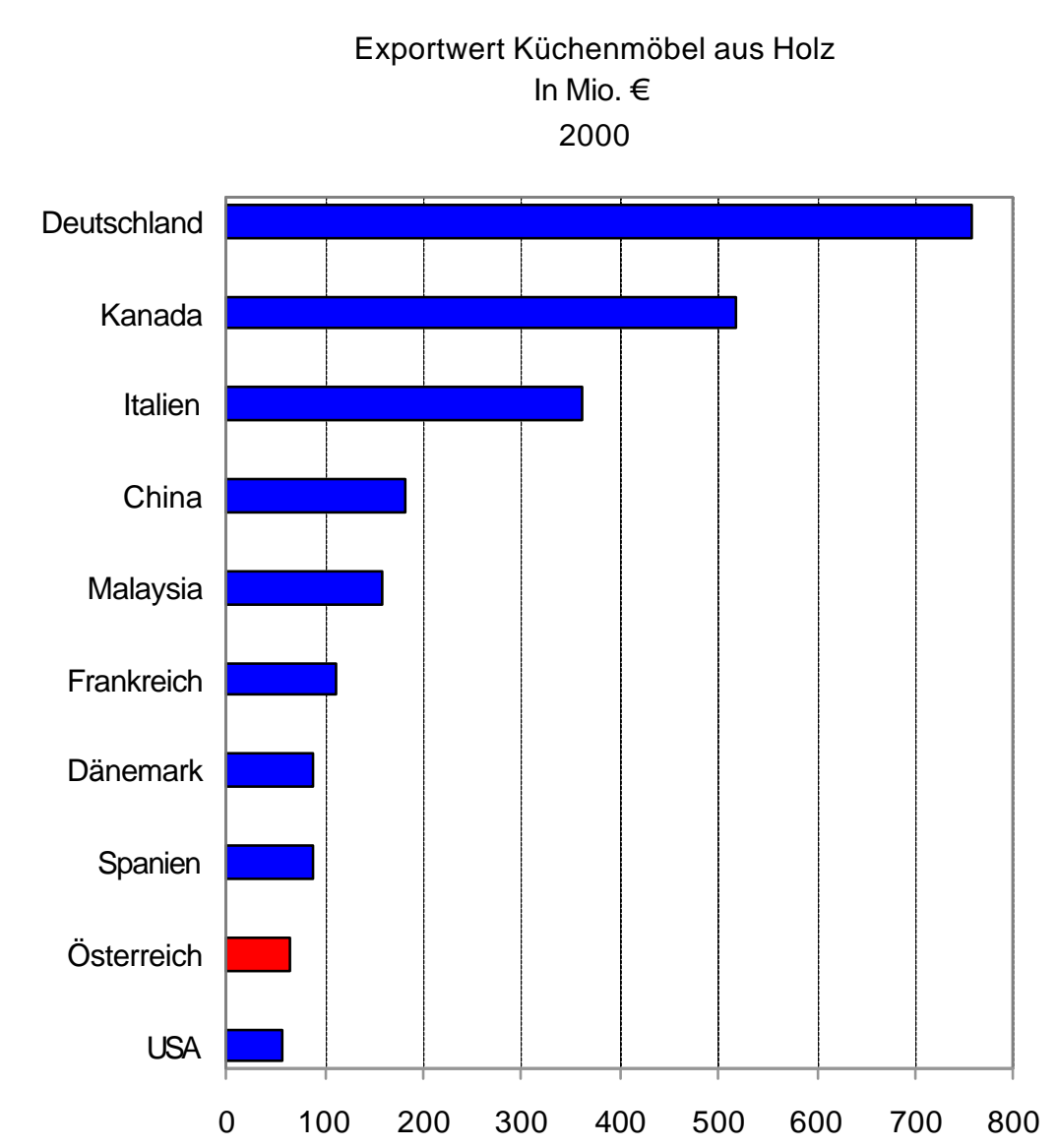


Abbildung 44: Dynamik der Exporte von

Schlafzimmermöbeln aus Holz

\begin{tabular}{|c|c|c|c|}
\hline SITC 82155 & Exportwert & & RCA-Wert \\
\hline & \multicolumn{2}{|c|}{ Durchschnittliches jährl. Wachstum in \% } & \\
\hline & $1990 / 1997$ & $1997 / 2000$ & 2000 \\
\hline Italien & $+11,8$ & $-0,5$ & $+2,622$ \\
\hline Kanada & $+26,9$ & $+22,3$ & $+1,528$ \\
\hline Dänemark & $+9,9$ & $+8,2$ & $+2,310$ \\
\hline Deutschland & $+1,7$ & $+1,5$ & $-0,285$ \\
\hline China & . & $+40,1$ & $+3,530$ \\
\hline Mexiko & $+58,9$ & $+16,4$ & $+2,057$ \\
\hline Brasilien & $+56,9$ & $+17,9$ & $+6,132$ \\
\hline Spanien & $+13,2$ & $+6,1$ & $+1,746$ \\
\hline Frankreich & $+8,4$ & $+7,0$ & $-0,629$ \\
\hline Polen & . & $+17,7$ & $+3,142$ \\
\hline Österreich & $+0,2$ & $-3,5$ & $-0,897$ \\
\hline
\end{tabular}

Quelle: UNO (WIFO-Datenbank)
Exportwert Schlafzimmermöbel aus Holz In Mio. $€$

2000

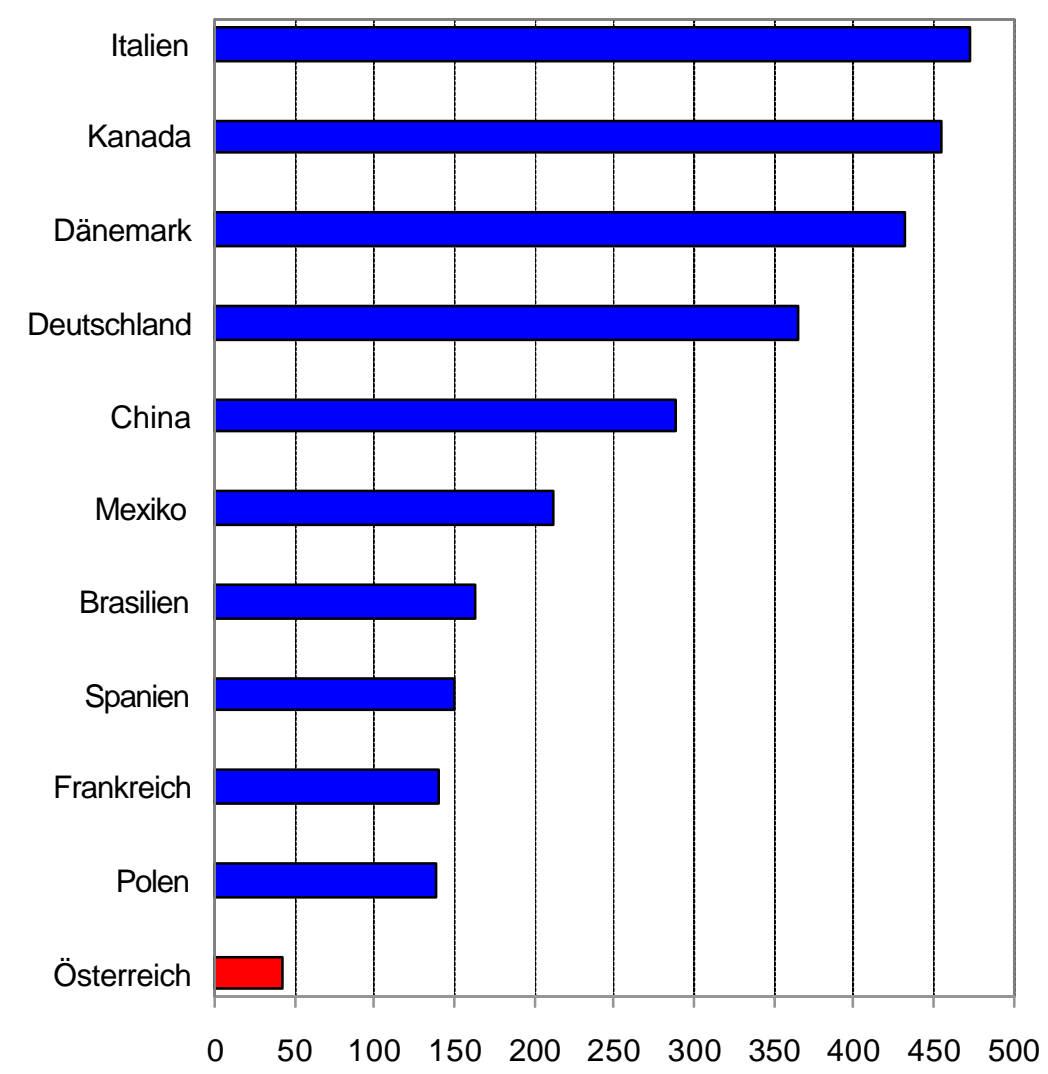


Abbildung 45: Dynamik der Exporte von anderen Möbeln aus Holz

\begin{tabular}{|c|c|c|c|}
\hline SITC 82159 & Exportwert & & RCA-Wert \\
\hline & \multicolumn{2}{|c|}{ Durchschnittliches jährl. Wachstum in \% } & \\
\hline & $1990 / 1997$ & $1997 / 2000$ & 2000 \\
\hline Italien & $+3,1$ & $+3,6$ & $+2,295$ \\
\hline China & . & $+28,6$ & $+4,999$ \\
\hline Kanada & $+22,6$ & $+27,9$ & $+0,631$ \\
\hline Dänemark & $+7,4$ & $-0,1$ & $+1,521$ \\
\hline Polen & . & $+10,1$ & $+3,106$ \\
\hline Malaysia & $+41,9$ & $+14,0$ & $+3,857$ \\
\hline Indonesien & $+52,8$ & $+41,5$ & $+6,199$ \\
\hline Deutschland & $+0,1$ & $+7,0$ & $-0,855$ \\
\hline Frankreich & $+7,4$ & $+14,1$ & $-0,362$ \\
\hline Großbritannien & $+3,0$ & $+7,2$ & $-0,485$ \\
\hline Österreich & $+7,4$ & $+2,7$ & $-0,716$ \\
\hline
\end{tabular}

Quelle: UNO (WIFO-Datenbank)

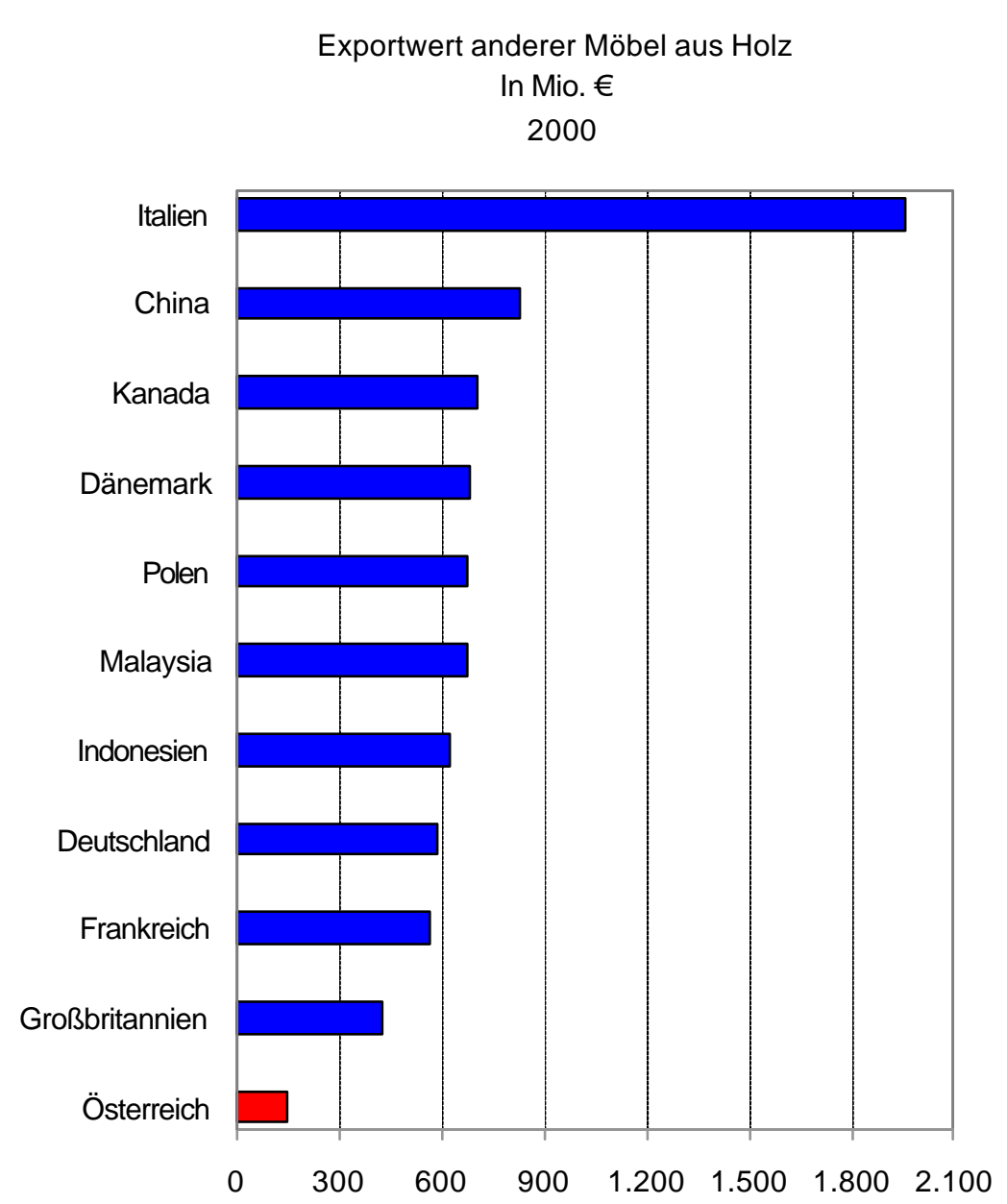


